\title{
Genetic and perinatal risk factors for movement disorders
}

Citation for published version (APA):

Barkhuizen, M. (2017). Genetic and perinatal risk factors for movement disorders. [Doctoral Thesis, Maastricht University, North-West University]. Maastricht University.

https://doi.org/10.26481/dis.20171204mb

Document status and date:

Published: 01/01/2017

DOI:

10.26481/dis.20171204mb

Document Version:

Publisher's PDF, also known as Version of record

\section{Please check the document version of this publication:}

- A submitted manuscript is the version of the article upon submission and before peer-review. There can be important differences between the submitted version and the official published version of record.

People interested in the research are advised to contact the author for the final version of the publication, or visit the DOI to the publisher's website.

- The final author version and the galley proof are versions of the publication after peer review.

- The final published version features the final layout of the paper including the volume, issue and page numbers.

Link to publication

\footnotetext{
General rights rights.

- You may freely distribute the URL identifying the publication in the public portal. please follow below link for the End User Agreement:

www.umlib.nl/taverne-license

Take down policy

If you believe that this document breaches copyright please contact us at:

repository@maastrichtuniversity.nl

providing details and we will investigate your claim.
}

Copyright and moral rights for the publications made accessible in the public portal are retained by the authors and/or other copyright owners and it is a condition of accessing publications that users recognise and abide by the legal requirements associated with these

- Users may download and print one copy of any publication from the public portal for the purpose of private study or research.

- You may not further distribute the material or use it for any profit-making activity or commercial gain

If the publication is distributed under the terms of Article $25 \mathrm{fa}$ of the Dutch Copyright Act, indicated by the "Taverne" license above, 


\section{Genetic and perinatal risk factors for movement disorders}

Melinda Barkhuizen 
(C) Melinda Barkhuizen, Maastricht, 2017

Genetic and perinatal risk factors for movement disorders

All rights are reserved. No part of this book may be reproduced or transmitted in any form or by any means, without permission from the copyright holder. IBSN:978-94-6295-822-7

Cover design and layout: Melinda Barkhuizen

Printing: Proefschriftmaken 


\title{
Genetic and perinatal risk factors for movement disorders
}

\author{
Dissertation
}

To obtain the degree of Doctor from Maastricht University (The Netherlands) and North-West University (South Africa)

in terms of a cotutelle agreement

On the authority of the Rector Magnifici prof.dr. R.M. Letschert and prof. F. Janse van Rensburg In accordance with the decision of the Board of Deans,

to be defended in public on Monday 4 December 2017 at 14:00 hours

by

Melinda Barkhuizen 


\section{Promoters:}

Prof.dr. B.W. Kramer

Prof.dr. H.W.M. Steinbusch

Prof. A.F. Grobler (North-West University, RSA)

\section{Co-Promoter:}

Dr. A.W.D. Gavilanes

\section{Assessment committee:}

Prof.dr. M.H. de Baets (Chairman, Maastricht University, NL)

Prof.dr. J. de Boer (Maastricht University, NL)

Prof. A.F. Kotze (North-West University, RSA)

Dr. M. Müller (Inselspital Bern, CH)

Prof.dr. J.B. Schulz (University Hospital Aachen, DE)

Prof. G. Terre'Blanche (North-West University, RSA)

Partial financial support for this thesis was kindly provided by the Sistema de Investigación y Desarrollo (SINDE) of the Universidad Católica de Santiago de Guayaquil, Guayaquil, Ecuador, through the grant No SIU- 319: Perinatal asphyxia and stem cell treatment and the National Research Foundation of South Africa (Grant specific reference numbers 89230 and 98217). 
'to my father

Soli Deo Gloria 


\begin{abstract}
Movement disorders, such as Parkinson's disease (PD), Huntington's disease (HD) and cerebral palsy (CP) are debilitating diseases with a great societal impact. These disorders are characterized by damage to the basal ganglia - a set of brain structures involved in motor coordination. The majority of PD cases are idiopathic and thought to be due to the cumulative effect of several genetic and environmental insults over a lifetime on the substantia nigra. In this thesis, I focused on risk factors for basal ganglia damage. I started by screening Caucasian South Africans with PD for variation in the GBA gene, encoding glucocerebrosidase. $12.38 \%$ of PD patients and $5.00 \%$ of controls in this population group carried a GBA variant, which is comparable to European populations.
\end{abstract}

Common environmental factors in South Africa that could interact with genetic factors to worsen basal ganglia damage were also investigated. SubSaharan Africa has a disproportionately high incidence of maternal complications and birth insults. Severe birth insults can lead to life-long disabilities such as $\mathrm{CP}$ and mental retardation. But even survivors without visible disability have an increased risk of delayed-onset neurological and psychiatric disorders. I investigated whether perinatal insults or neurodevelopmental disorders could decrease the age-of-onset of $\mathrm{HD}$, a fatal, inherited movement disorder caused by the death of medium spiny neurons in the striatum. Both insults reduced age-of-diagnosis of HD in two large epidemiological studies. In a rat model of preterm perinatal asphyxia (PA), the insult selectively increased nitric oxide production, and thus nitrosidative stress, in the striatum within the first week after the insult. This could possibly contribute to the phenotype seen in HD patients with perinatal insults.

The last part of my thesis focused on therapeutic interventions for PA in the preterm brain. PA causes a massive release of glutamate, which leads to excitotoxicity and neuronal death. I tested whether antagonism of the glutamatergic-NMDA receptors with the anesthetics propofol and isoflurane could protect the substantia nigra. Anesthesia was given to the maternalfetal unit of a sheep model of PA due to umbilical cord occlusion. Propofol 
anesthesia limited dopaminergic neuron loss, but this was associated with increased tau-phosphorylation in several brain regions which could potentially be detrimental. I further examined the efficacy of multipotent adult progenitor cells (MAPC) therapy for PA in the immature rat brain. The administration of two doses within 24 hours after the insult had a long-lasting effect on gene transcription in the two-week old rat brain. The effects on gene transcription depended on whether the cells were administered through the intranasal or systemic route. The systemic administration had a partial beneficial effect on recognition memory and locomotion in adult rats exposed to PA at birth.

In conclusion, this thesis contributes to the body of knowledge on the influence of perinatal and genetic insults on the basal ganglia and the resulting movement disorders, as well as therapeutic strategies to attenuate neuronal loss and functional disabilities.

Keywords: Movement disorder, basal ganglia, Parkinson's disease, Huntington's disease, neurogenetics, risk factor, epidemiology, perinatal asphyxia, preterm, stem cell therapy 


\section{Preface:}

This doctoral thesis is submitted in article format, consisting of:

- Two peer-reviewed published reviews:

- Chapter 2: Neurochemistry International, 93(2016):6-25

- Chapter 5: Neuroscience \& Biobehavioral Reviews, 75(2017):166-182

- Two peer-reviewed published origional articles:

- Chapter 3: Molecular Genetics and Genomic Medicine, 5.2(2017):147-156

- Chapter 6: Neurotoxicity Research, 31.3(2017):400-409

- Two original articles that have been submitted to peer-reviewed journals:

- Chapter 4: Submitted to Journal of Neurology, Neurosurgery and Psychiatry

- Chapter 7: Submitted to Pediatric Research

- The remaining two chapters (8 and 9) are presented as manuscripts in preparation for submission

- Permission has been granted by the relevant journals and all co-authors to include these articles in this doctoral thesis. 
I, Ms. Melinda Barkhuizen, hereby declare that this disseration is a record of my own work (except where citations or acknowledgements indicate otherwise) and that the study in part or as a whole has not been submitted to any other universities apart from the Maastricht University (Netherlands) and the North-West University (South Africa).

I would like to acknowledge the following individuals or organizations for their contributions to the work described in this thesis:

Parkinson's disease genetic study The participants were collected through a network of referring doctors and neurologists in South Africa. AMPATH laboratories graciously facilitated blood collection, Inqaba biotec conducted the genotyping and Mr. Iain Sinclair at the National Health Laboratory Service conducted the enzymatic activity testing

Huntington's disease epidemiology The statistical analyses were designed by Dr. Bjorn Winkens and Dr. Filipe Broguiera Rodrigues. Huntington's disease patient data was supplied by the Enroll-HD and European Huntington's Disease Network REGISTRY studies

Rat brain tissue The rat perinatal asphyxia experiments were conducted with the help of Dr. Wilma van de Berg, Mr. Ralph van Mechelen, Dr. Danilo Gavilanes and Ms. Marijne Vermeer. The multipotent adult progenitor cells were provided by Dr. Robert Mays and Dr. Bart Vaes from Athersys

Sheep brain tissue The sheep umbillical cord occlusion experiments were conducted by Dr. Matthias Seehase, Dr. Reint Jellema, Dr. Ruth Gussenhoven and Prof.dr. Boris Kramer

Histology and microscopy The histology and microscopy described in this thesis conducted with the help of Dr. Wilma van de Berg, Ms. Fleur van Dijck and Ms. Imke Engelbertink. Dr. Jack Cleutjens wrote a script to quantify neurons. The lab of Prof. Peter Davies donated the anti-tau antibodies used

Transcriptomics The transcriptome and analysis was performed at the Toxicogenomics department with the help of Mr. Marcel van Herwijnen and Dr. Danyel Jennen

Rat behavior testing The behavior testing was conducted and scored with the help of Mr. Ralph van Mechelen, Ms. Marijne Vermeer and Mr. Dean Paes 
Manuscript My co-authors edited the drafts and they, along with several others, contributed invalubale advice which added to the intellectual content of this thesis. Please see the full acknowledgments at the end of this thesis

Funding Partial financial support for this thesis was provided by the Sistema de Investigación y Desarrollo (SINDE) of the Universidad Católica de Santiago de Guayaquil, Guayaquil, Ecuador, through the grant No SIU- 319: Perinatal asphyxia and stem cell treatment and the National Research Foundation of South Africa (Grant specific reference numbers 89230 and 98217), the DST/NWU Preclinical Drug Development Platform and the Stichting Bevordering Kindergeneeskunde 




\section{CONTENTS}

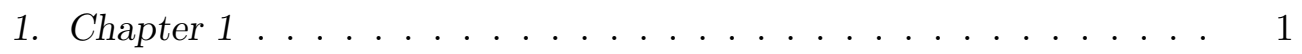

General introduction . . . . . . . . . . . . . . . . . 2

1.1 The movement disorders . . . . . . . . . . . . . . 2

1.2 Perinatal asphyxia . . . . . . . . . . . . . 4

2. Chapter $2 \ldots \ldots \ldots$

Advances in GBA-associated Parkinson's disease - pathology, presentation and therapies

Neurochemistry international, 2016, $93,6-25 \ldots \ldots \ldots$

2.1 Introduction . . . . . . . . . . . . . . . . . . . . 12

2.2 Molecular functions of $G B A \ldots \ldots \ldots \ldots$

2.3 Clinical pictures for GBA-PD . . . . . . . . . . . 33

2.4 Discussion and conclusion . . . . . . . . . . . . . . . . 43

2.5 Acknowledgements . . . . . . . . . . . . . 47

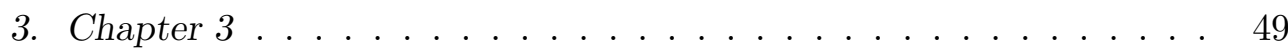

A molecular analysis of the $G B A$ gene in Caucasian South Africans with Parkinson's disease. Molecular Genetics \& Genomic Medicine, 2017,

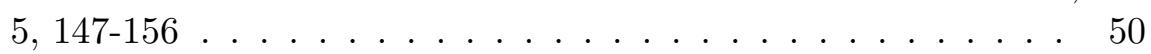

3.1 Introduction . . . . . . . . . . . . . . . . . 51

3.2. Materials and methods . . . . . . . . . . . . . 53

3.3 Results . . . . . . . . . . . . . . . . 55

3.4 Discussion . . . . . . . . . . . . . . . . . . 60

3.5 Conclusion . . . . . . . . . . . . . . 63

3.6 Acknowledgements . . . . . . . . . . . . . . . . . . . . . . . . . . 63

3.7 Supplementary material . . . . . . . . . . . . . . . 64

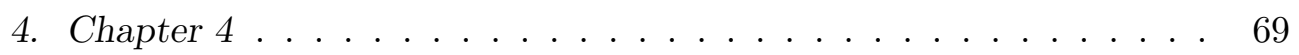

Perinatal insults and neurodevelopmental disorders may impact age

of diagnosis of Huntington's disease. Submitted . . . . . . . . 70

4.1 Introduction . . . . . . . . . . . . . . . . . . . . . 72

4.2 Materials and methods . . . . . . . . . . . . . 73 
4.3 Results . . . . . . . . . . . . . . . . . . . . . . 77

4.4 Discussion and conclusions $\ldots \ldots \ldots . . \ldots 79$

4.5 Acknowledgements . . . . . . . . . . . . . . . . . 83

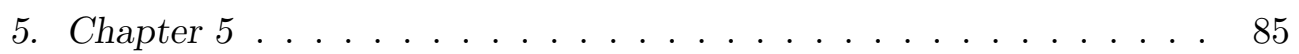

25 Years of research on global asphyxia in the immature rat brain

Neuroscience \& Biobehavioral Reviews, 2017, 75, 166-182 . . . 86

5.1 Introduction . . . . . . . . . . . . . . . . 87

5.2 The pathological effects of PA . . . . . . . . . . . . 91

5.3 The functional effects of PA . . . . . . . . . . . . . . 95

5.4 The molecular effects of PA on neurotransmitter systems . 101

5.5 Translational aspects . . . . . . . . . . . . . 117

5.6 Discussion and conclusion . . . . . . . . . . . . . 122

5.7 Acknowledgements . . . . . . . . . . . . . . . 124

6. Chapter 6 . . . . . . . . . . . . . . . . . . . 127

Nitric oxide production in the striatum and cerebellum of a rat model of preterm global perinatal asphyxia. Neurotoxicity research, $2017,31,400-409 \ldots \ldots \ldots \ldots \ldots \ldots$

6.1 Introduction . . . . . . . . . . . . . . . . . . 129

6.2 Materials and methods . . . . . . . . . . . . . 132

6.3 Results . . . . . . . . . . . . . . . . 136

6.4 Discussion . . . . . . . . . . . . . . . . . . . . . . . . . 141

6.5 Conclusion $\ldots \ldots \ldots \ldots \ldots \ldots$

6.6 Acknowledgements . . . . . . . . . . . . . . . 145

7. Chapter 7 . . . . . . . . . . . . . . . . . . 147

The influence of anesthetics on tyrosine hydroxylase expression and tau phosphorylation in the hypoxic-ischemic near-term lamb.

Submitted . . . . . . . . . . . . . . . . . . 148

7.1 Introduction . . . . . . . . . . . . . . . . 150

7.2 Materials and methods . . . . . . . . . . . . . . . 152

7.3 Results . . . . . . . . . . . . . . . 156

7.4 Discussion . . . . . . . . . . . . . . . . 163

7.5 Conclusion . . . . . . . . . . . . . . 166

7.6 Acknowledgments $\ldots \ldots \ldots \ldots 6$

8. Chapter 8 . . . . . . . . . . . . . . . . . . . . . . . 169

The transcriptional effects of multipotent adult progenitor cells in a rat model of preterm perinatal asphyxia. In preparation . . . . 170

8.1 Introduction . . . . . . . . . . . . . . . . . 172 
8.2 Materials and methods . . . . . . . . . . . . . . . 174

8.3 Results . . . . . . . . . . . . . . . . 177

8.4 Discussion . . . . . . . . . . . . . . . . . . . . 182

8.5 Conclusion . . . . . . . . . . . . . . . 187

8.6 Acknowledgements . . . . . . . . . . . . . . 187

9. Chapter 9 . . . . . . . . . . . . . . . . . . . . . . . . . 189

Long-term functional outcomes of multipotent adult progenitor cell therapy for asphyxia-related encephalopathy in the immature rat brain. In preparation. . . . . . . . . . . . . . . 190

9.1 Introduction . . . . . . . . . . . . . . . . . 192

9.2 Materials and methods . . . . . . . . . . . . . . . . . . 193

9.3 Results . . . . . . . . . . . . . . . . . 197

9.4 Discussion . . . . . . . . . . . . . . . . . 203

9.5 Conclusion . . . . . . . . . . . . . . . 208

9.6 Acknowledgements . . . . . . . . . . . . . . . . 209

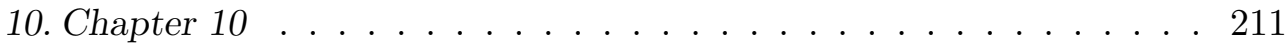

General discussion and summary . . . . . . . . . . . . . . . . 212

10.1 General pathological hallmarks of age-related neurodegenerative diseases and the overlap with perinatal asphyxia 212

10.1 .1 Discussion . . . . . . . . . . . . . . . . 212

10.1 .2 Summary . . . . . . . . . . . . . . . . . 215

10.2 Therapeutic interventions . . . . . . . . . . . . 217

10.2 .1 Discussion . . . . . . . . . . . . . . . . . . 217

10.2 .2 Summary . . . . . . . . . . . . . . . . . . 217

10.3 Nederlandse samenvatting . . . . . . . . . . . . . . . 220

10.4 Afrikaanse opsomming . . . . . . . . . . . . . . . . . 223

11. Appendix-Valorization . . . . . . . . . . . . . . . 227

12. Appendix - References . . . . . . . . . . . . . . . . . . 233

13. Appendix - Affiliations of co-authors . . . . . . . . . . . . . . . 281

14. Acknowledgements and about the author . . . . . . . . . . . . 285

Acknowledgements . . . . . . . . . . . . . . . 285

Curriculum Vitae . . . . . . . . . . . . . . . . . . . . . . . . 293 


\section{LIST OF TABLES}

Table 2.1: The frequency of $G B A$ mutations in Parkinsonian syndromes and other neurodegenerative diseases. . . . . . . . . . . 41

Table 2.2 Gaucher disease populations screened since 2014 . . . . . . 43

Table 2.3: Parkinson's disease populations screened for $G B A$ mutations since 2014. . . . . . . . . . . . . . . . . 43

Table 3.1: Non-synonymous substitutions identified . . . . . . . . 56

Table 3.2: Leukocyte glucocerebrosidase activity and pathogenicity predictions of the substitutions identified. . . . . . . . 60

Supplementary table 3.1: Synonymous and intronic variants identified 67

Supplementary table 3.2: - MutPred predictions of functional consequences of the mutations (Li et al., 2009) . . . . . . . . . 67

Table 4.1: Description of the comorbidities included from the REGISTRY and Enroll-HD cohorts, divided into perinatal insults and neurodevelopmental disorders. . . . . . . . . . . . . 77

Table 4.2: Characteristics of the merged cohort, the REGISTRY cohort, and the Enroll-HD cohort. . . . . . . . . . 79

Table 4.3: Survival differences by gender and affected parent per group. 79

Table 5.1: An overview of behavioural changes seen in male rats relative to controls . . . . . . . . . . . . . . . 101

An overview of changes in neurotransmission in the SN/VTA and

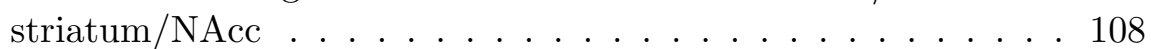

Table 6.1: Summary of the experiments performed with rat striatal slices at postnatal day $(\mathrm{P}) 5, \mathrm{P} 8$ and $\mathrm{P} 12 . \ldots \ldots 136$

Table 8.1: Enriched pathways constructed from Differentially Expressed Genes . . . . . . . . . . . . . . . . . 178 


\section{LIST OF FIGURES}

Figure 1.1: The central hypotheses . . . . . . . . . . . . 5

Figure 2.1: Pathways of the altered ceramide metabolites . . . . . . 31

Figure 2.2: An overview of the GBA-PD neuropathological cascade. 31

Figure 3.1: Important interactions between $\beta$-glucocerebrosidase and $\alpha$-synuclein at $\mathrm{pH} 5.5 . \ldots \ldots \ldots \ldots \ldots$

Figure 3.2: Overall conformation of the substituted $\beta$-glucocerebrosidase receptor after minimization for docking with $\alpha$-synuclein at $\mathrm{pH}$ 5.5 and $\mathrm{pH} 7 . \ldots \ldots \ldots \ldots \ldots \ldots \ldots \ldots$

Supplementary figure 3.1: The top-poses of the $\alpha$-synuclein side-chain docked into substituted $\beta$-glucocerebrosidase $\ldots . . . . . .664$

Supplementary figure 3.2: A magnified view of the conformation of selected substituted $\beta$-glucocerebrosidase receptors at $\mathrm{pH} 5.5$ docked with $\alpha$-synuclein. . . . . . . . . . . . . . . . . . 64

Figure 4.1: Flow chart of included participants and exclusions. . . . 74

Figure 4.2: Kaplan-Meier survival estimates of the age of diagnosis for the merged cohort. . . . . . . . . . . . . . 77

Figure 5.1: Graphical abstract . . . . . . . . . . . . 86

Figure 6.1: The study design ． . . . . . . . . . . . . . . . . . 132

Figure 6.2: DAF-2 fluorescence in tissue slices from medial striatum and cerebellum of a control and asphyctic rat at postnatal day 8.136

Figure 6.3: DAF-2 fluorescence in tissue slices of the medial striatum and cerebellum of a control rat . . . . . . . . . . . 136

Figure 6.4: Double-labelling of parvalbumin and NO-production markers, cGMP or nNOS . . . . . . . . . . . . . . . . 139

Figure 6.5: Caspase-3-like activity within the cerebellum of control and asphyctic rats during the first 15 days after birth. . . . . . 141

Figure 6.6: Histogram of division of pixels over intensity classes of GFAP immunoreactivity in striata of control and asphyctic rats. 141 
Figure 7.1: The cumulative TH-neuron counts in the substantia nigra over 4 slides. . . . . . . . . . . . . . 156

Figure 7.2: Representative photos of the TH-staining in the substantia nigra at $4 \mathrm{x}$ and $40 \mathrm{x}$ magnification. . . . . . . . . . . . 156

Figure 7.3: A comparison of the TH, IBA-1 and CP-13 immunohistochemistry in the substantia nigra of an UCO-I lamb at 2x and 10x magnification. . . . . . . . . . . . . . 158

Figure 7.4: Microglial proliferation in the thalamus and stratum moleculare (S.M.) layer below the dentate gyrus of the hip-

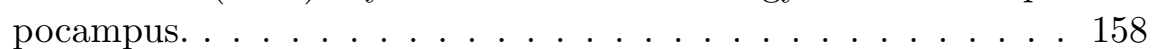

Figure 7.5: The intensity of anti-phosphorylated (ser-202) tau immunohistochemistry in the substantia nigra and thalamus. . . . 161

Figure 7.6: The intensity of anti-phosphorylated (ser-202) tau immunohistochemistry in the hippocampus CA3 and DG layers. . 161

Figure 8.1: Differentially expressed genes with a fold-change $>1.2$ and $\mathrm{p}<0.05$ versus vehicle-treated PA per group. . . . . . . . . 177

Figure 8.2: Amount of enriched gene-ontology terms per gene-ontology group constructed with the differentially expresed genes for each

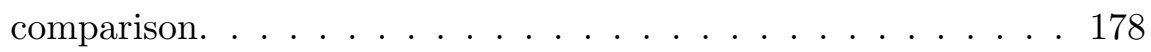

Figure 8.3: The PA network and the effect of the MAPC therapy. . 178

Figure 9.1: Weight gained over the course of the study. . . . . . . . . 197

Figure 9.2: The total distance moved in 40 minutes. . . . . . . . . . 198

Figure 9.3: The discriminatory index after 4 hours delay. . . . . . . 199

Figure 9.4: The results of the elevated zero maze. . . . . . . . . . . 199

Figure 9.5: The home-cage emergence task . . . . . . . . . . . . . 200

Figure 9.6: The results of overall sociability in the social interaction test. ........................... 203

Figure 9.7: The results of sniffing in the social interaction test. . . . 203

Figure 10.1: The genetic- environmental continuum of basal ganglia insults studied. . . . . . . . . . . . . . . . 214 




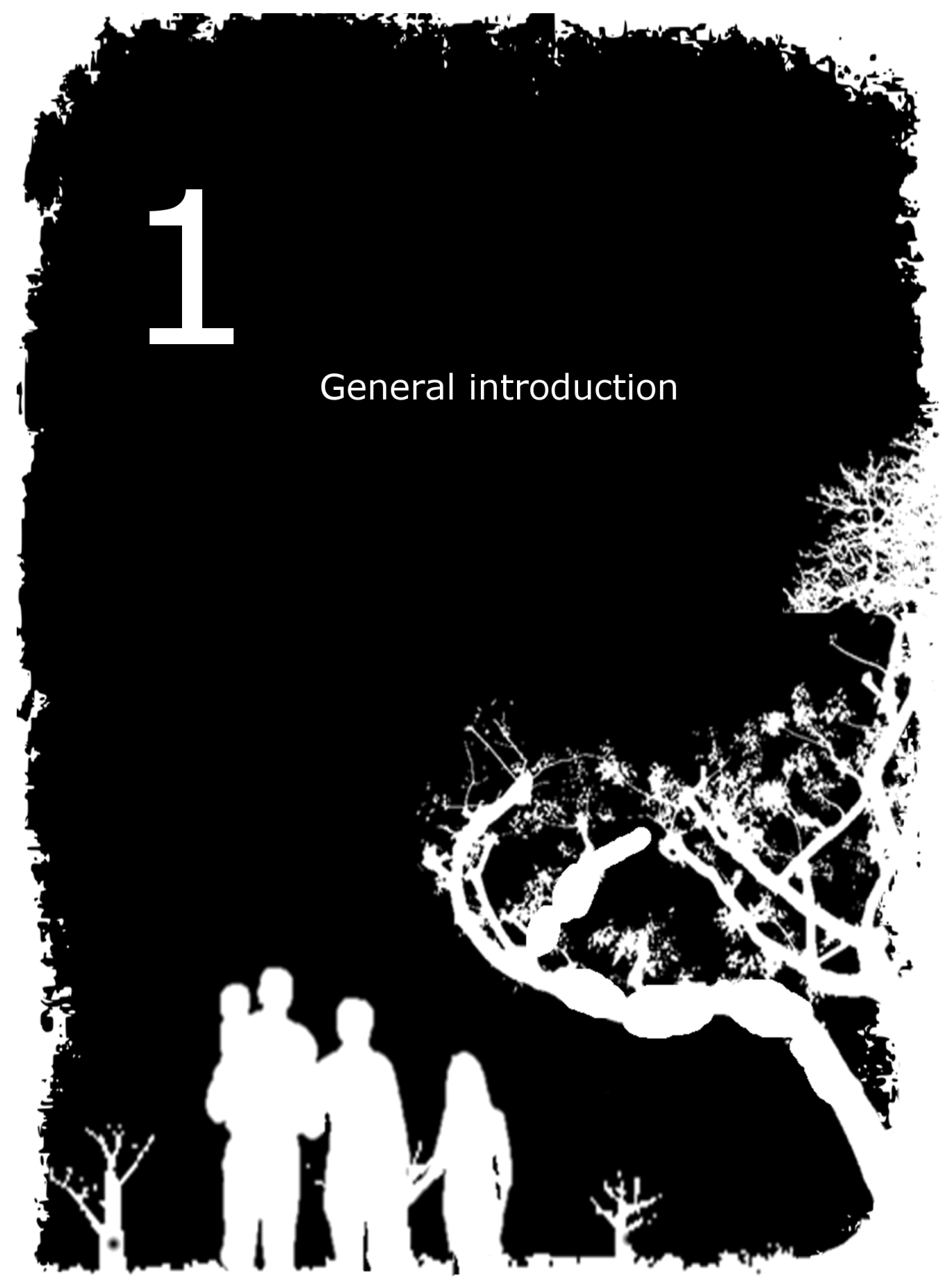




\section{General introduction}

\subsection{The movement disorders:}

Parkinson's disease (PD) is a progressive neurodegenerative disease characterized by a triad of motor symptoms consisting of tremor, rigidity, and akinesia. Non-motor symptoms like constipation, depression, loss-of-smell, psychosis and cognitive decline also cause a considerable burden in $\mathrm{PD}$ and often precede the motor symptoms (Chaudhuri, Healy et al. 2006).

PD and other movement disorders, such as Huntington's disease (HD), are a substantial global health burden. PD is the second most common neurodegenerative disease globally, after Alzheimer's disease (Dauer and Przedborski 2003). In 2005, PD affected 4.1-4.6 million individuals over the age of 50 in the world's most populous nations. This number is expected to increase in the coming decades (Dorsey, Constantinescu et al. 2007). There is an urgent need to reduce the burden of PD by modifying risk factors and identifying individuals with pre-symptomatic disease for enrollment in clinical trials aimed at preventing or delaying the disease (Salat, Noyce et al. 2016). In order to identify at-risk individuals earlier, a better understanding of genetic, environmental and neurodevelopmental factors which may contribute to the disease is needed.

A small percentage of PD cases is entirely caused by either genetic or environmental factors, like exposure to large doses of organophosphates or heavy metals (Goldman 2014). Thus far, a number of Mendelian causes of PD have been identified, including rare mutations in the autosomal dominant genes $S N C A$ (which encodes alpha-synuclein), LRRK2 and recessive genes PARK2, PINK1, DJ1, PLA2G6, FBXO7, and ATP13A2. Together, the Mendelian genes account for less than 10\% of PD cases (Singleton, Farrer et al. 2013). While it is known that some of the mutations in Mendelian genes will inevitably lead to neurodegenerative disease if the person lives long enough, like SNCA triplications, most of the Mendelian genes have an incomplete age-dependent penetrance, which can differ between mutations in a gene and ethnicity (Singleton, Farrer et al. 2013, Trinh, Guella et al. 2014). Genetic risk factors for PD include common genetic variation in SNCA, LRRK2, MAPT (which encodes tau), as well as mutations in the glucocerebrosidase $(G B A)$ gene, which is the most common large-effect genetic risk factor for PD (Singleton, Farrer et al. 2013, Kim, Cintron et al. 2015, Lesage 2015, Spataro, Calafell et al. 2015). 
HD is an incurable autosomal dominant disorder caused by a CAG trinucleotide repeat expansion in an intron of the HTT gene, which encodes huntingtin. Expansions greater than 40 repeats have a complete penetrance. HD has an average disease course of 20 years after clinical onset and usually presents in early mid-life with abnormal movements (particularly chorea) together with psychiatric symptoms; including psychosis, depression, and obsessive-compulsive disorder; and progressive cognitive impairment (Rawlins, Wexler et al. 2016). $\mathrm{HD}$ is rare, with an average prevalence of 3.6 cases per 100000 in Western Europe, but it has a substantial direct economic burden (Squitieri, Griguoli et al. 2015, Rawlins, Wexler et al. 2016). The length of the CAG repeat expansion is the primary predictor of age-of-onset of disease. However, there is substantial phenotypic variability among HD gene expansion carriers and other genetic factors can hasten or delay the clinical onset of disease (Lee, Wheeler et al. 2015). The age-of-onset can also be modified by environmental factors like passivity (Trembath, Horton et al. 2010). Environmental factors may play a larger modifying role than co-existent genetic factors (Wexler 2004).

On a neuropathological level, both these diseases are associated with the selective neuronal death within components of the basal ganglia, which spreads across the brain as the diseases progress. The basal ganglia consist of a group of interconnected and functionally related nuclei located in the forebrain and the midbrain namely: the striatum, globus pallidum, subthalamic nucleus, and substantia nigra (Middleton and Strick 2000). In PD, neuronal death in the substantia nigra is associated with the accumulation of insoluble protein aggregates, like alpha-synuclein and tau (Moussaud, Jones et al. 2014). In $\mathrm{HD}$, striatal neuropathology is primarily caused by huntingtin aggregates and secondary pathologies like tau (Fernández-Nogales, Cabrera et al. 2014).

PD and HD both have a long latent phase with symptoms of progressive neurodegeneration only occurring years after the damage has been initiated. In HD, the causative CAG-repeat expansion is present from conception, but the disease normally manifests in early mid-life (Rawlins, Wexler et al. 2016). In $\mathrm{PD}$, it is estimated that symptoms only occur once $60-80 \%$ of the nigral dopaminergic neurons have already been lost (Dauer and Przedborski 2003). Thus, there is a window-of-opportunity of several years before symptom presentation to intervene.

It has been postulated that the majority of sporadic PD cases are due to the cumulative effect of multiple genetic and environmental 'hits' to the dopaminergic neurons. Cumulatively these 'hits' cross the threshold for neu- 
ronal death (Sulzer 2007). The first hit may occur early in development. The basal ganglia are particularly prone to structural damage early in life, due to their high energy requirements (Bekiesinska-Figatowska, Mierzewska et al. 2013). Trauma in the perinatal period initiates a long-lasting metabolic or neurodegenerative cascade that continues for a considerable period of time before diagnosable symptoms appear. Neurological damage originating in the perinatal period could manifest in childhood, i.e. cerebral palsy and seizures, or in adolescence and young adulthood, i.e. schizophrenia (Marriott, RojasMancilla et al. 2015). Perinatal and early-life insults also cause deficient maturation that results in a less well-developed brain, with diminished reserve capacity (Seifan, Schelke et al. 2015), as well as lasting changes in epigenetic regulation and gene expression through life, which increases susceptibility to late-life neurodegenerative diseases (Lahiri and Maloney 2010).

\subsection{Perinatal asphyxia:}

Perinatal asphyxia (PA) is a common cause of brain damage in the period around birth. Hypoxic-ischemic encephalopathy due to PA occurred in 8.5 infants per 1000 live births in 2010, amounting to 1.15 million infants globally (Lee, Kozuki et al. 2013). In the same period, 14.9 million infants were born preterm (Blencowe, Cousens et al. 2012). The patterns of brain injury vary according to the maturity of the brain at the time of the insult. Preterm infants can have encephalopathy due to the brain's maturation state, in the absence of additional insults. Hypoxic-ischemia during this time adds insult to an already vulnerable system. Factors like the degree of maturity of the brain, the selective ischemic vulnerability of developing neuron subpopulations, the severity of the insult and the characteristics of the reperfusion after hypoxicischemia determine the severity of the damage after PA in the preterm brain (Alvarez-Diaz, Hilario et al. 2007, Volpe 2009a, Volpe 2009c).

In preterm infants, injury of the sub-cortical white matter (termed periventricular leukomalacia) is the major site of damage, whilst term survivors often have a bilateral injury to the basal ganglia, including the striatum and substantia nigra, and the cerebellum. However, periventricular leukomalacia rarely occurs in isolation in preterm infants. These infants often also have grey matter injury to the basal ganglia and thalamus. Some infants also have brain stem injury (Volpe 2012, Bekiesinska-Figatowska, Mierzewska et al. 2013, Pagida, Konstantinidou et al. 2013). Functional outcomes vary according to the degree of asphyxia and overlap with some of the domains affected by movement disorders (Volpe 2012). Children with mild encephalopathy generally 


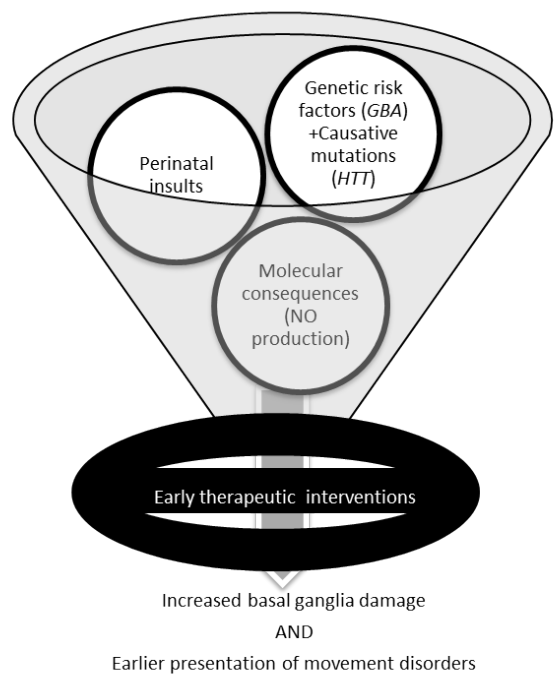

Figure 1.1: The central hypotheses

do not have overt neurological impairments, like mental or motor retardation at preschool age, but they may have subtle learning difficulties. In contrast, children with severe encephalopathy nearly always die or develop severe basal ganglia-associated motor impairments like cerebral palsy, as well as mental retardation, epilepsy and in some cases sensorineural hearing loss or cortical visual impairment. (Van Handel, Swaab et al. 2007). Very-low birth weight infants make up a large proportion of this disability burden. With improved medical care, a larger proportion of these infants survives up to adulthood and old-age (Volpe 2009a).

The central hypotheses investigated in this thesis are that: perinatal insults can aggravate movement disorders, and early therapeutic interventions can reduce basal ganglia damage and long-term functional impairment.

In this thesis, I investigated genetic and perinatal factors that are associated with basal ganglia damage, as well as therapeutic strategies to limit the destruction. In the first chapters, I investigated a genetic risk factor for neurodegeneration. In chapter $\mathbf{2}$, the mechanisms of how variations in the $G B A$ gene increase the risk for PD and affect disease onset and progression were reviewed. In chapter 3, I determined the prevalence of $G B A$ mutations in a South African cohort with PD. 
Subsequently, I investigated the interaction between genetic factors and perinatal insults in movement disorders. In chapter 4 , I investigated whether perinatal insults and neurodevelopmental disorders affect the onset of HD in two large epidemiological cohorts.

In chapter 5, the molecular cascades, neurotransmitter deregulation, and functional impairments after PA in the immature brain of a rat model were reviewed. In chapter $\mathbf{6}$, the timing of increased nitric oxide production after PA in the striatum and cerebellum was investigated. These regions are known to be vulnerable to PA and HD (Volpe 2009, Rüb, Vonsattel et al. 2015). Nitric oxide is involved in the pathological cascade after PA (Perlman 2006). Knowing the timing of NO release could aid in therapeutic development. Subsequently, therapeutic interventions to limit the basal ganglia damage after PA were investigated.

In chapter 7, I investigated whether limiting excitotoxicity with anesthetics could prevent neuron loss in the substantia nigra with a sheep model of PA. It was futher determined whether the anesthesia therapy increased tau-hyperphosphorylation - a precursor to tau pathology. Tau pathology is a hallmark of several neurodegenerative diseases (Compta, Parkkinen et al. 2014).

In chapter 8, I investigated whether multipotent adult progenitor cells (MAPCs) could normalize changes in gene transcription after PA. MAPCs are a stem cell product with immunomodulatory effects (Bedi, Hetz et al. 2013). In chapter 9, it was investigated whether the MAPC therapy could prevent the behavioral deficits seen after PA. In chapter 10, I summarized and discussed the main findings of this thesis.

This thesis contributes to the body of knowledge on the influence of perinatal and genetic insults on the basal ganglia and the resulting movement disorders, as well as therapeutic strategies to attenuate neuronal loss and functional disabilities. 




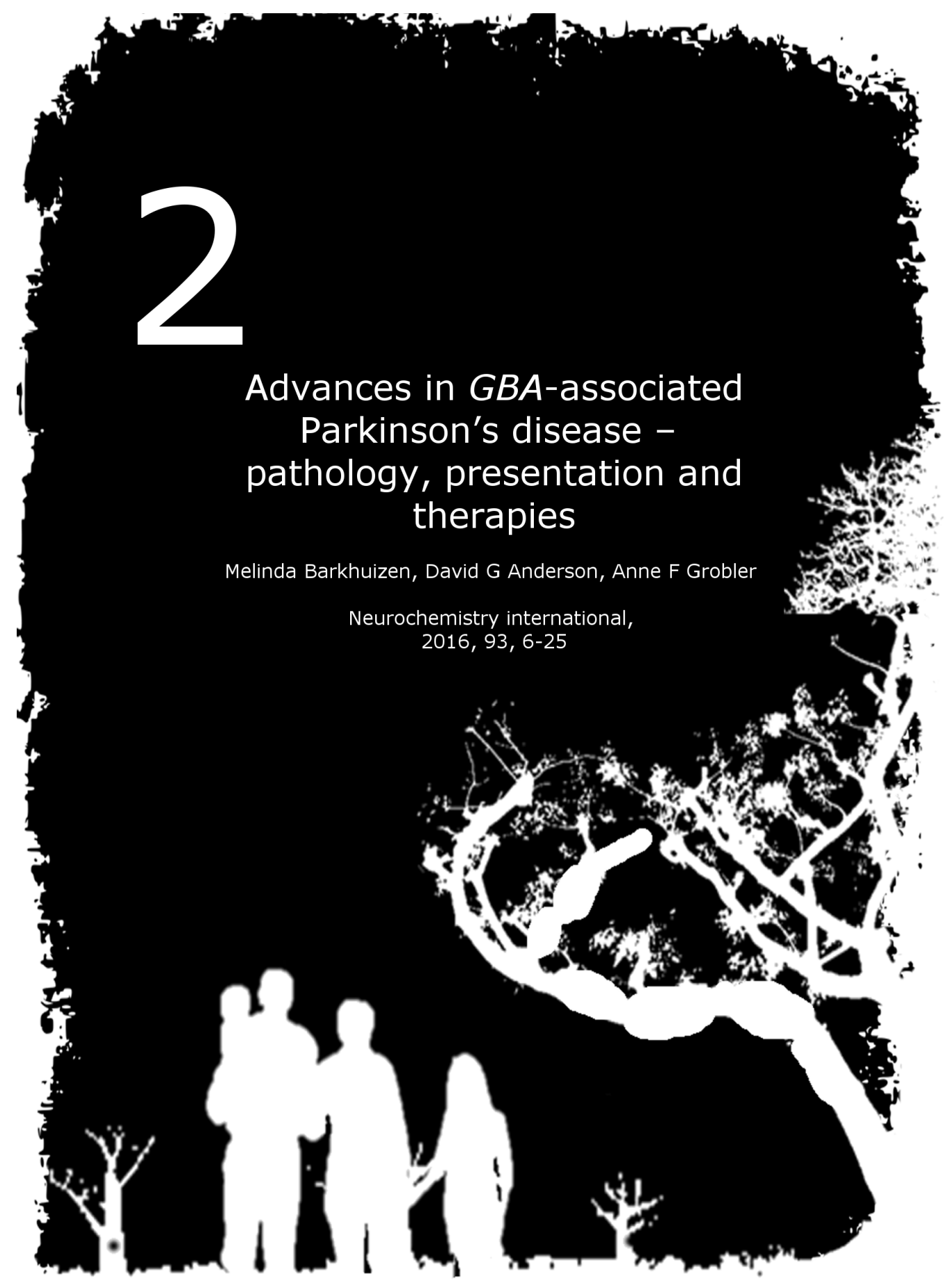




\title{
Advances in $G B A$-associated Parkinson's dis- ease - pathology, presentation and therapies
}

\author{
Melinda Barkhuizen, David G. Anderson, Anne F. Grobler \\ Neurochemistry international, 2016, 93, 6-25
}

\begin{abstract}
:
$G B A$ mutations are to date the most common genetic risk factor for Parkinson's disease. The GBA gene encodes the lysomal hydrolase glucocerebrosidase. Whilst bi-allelic GBA mutations cause Gaucher disease, both mono- and biallelic mutations confer risk for Parkinson's disease. Clinically, Parkinson's disease patients with $G B A$ mutations resemble idiopathic Parkinson's disease patients. However, these patients have a modest reduction in age-of-onset of disease and a greater incidence of cognitive decline. In some cases, GBA mutations are also responsible for familial Parkinson's disease. The accumulation of $\alpha$-synuclein into Lewy bodies is the central neuropathological hallmark of Parkinson's disease. Pathologic GBA mutations reduce enzymatic function. A reduction in glucocerebrosidase function increases $\alpha$-synuclein levels and propagation, which in turn inhibits glucocerebrosidase in a feed-forward cascade. This cascade is central to the neuropathology of $G B A$-associated Parkinson's disease. The lysosomal integral membrane protein type-2 (LIMP-2) is necessary for normal glucocerebrosidase function. Glucocerebrosidase dysfunction also increases in the accumulation of $\beta$-amyloid and amyloid-precursor protein, oxidative stress, neuronal susceptibility to metal ions, microglial and immune activation. These factors contribute to neuronal death. The Mendelian Parkinson's disease genes, Parkin and ATP13A2, intersect with glucocerebrosidase. These factors sketch a complex circuit of $G B A$-associated neuropathology. To clinically interfere with this circuit, central glucocerebrosidase function must be improved. Strategies based on reducing breakdown of mutant glucocerebrosidase and increasing the fraction that reaches the lysosome has shown promise. Breakdown can be reduced by interfering with the ability of heat-shock proteins to recognize mutant glucocerebrosidase. This underlies the therapeutic efficacy of certain pharmacological chaperones and histone deacetylase inhibitors. These therapies are promising for Parkinson's disease, regardless of mutation status. Recently, there has been a boom in studies investigating the role of GCase in the pathology of PD. This merits a comprehensive review of the current cell biological processes and pathological pictures involving Parkinson's disease associated with $G B A$ mutations.
\end{abstract}


Keywords: Glucocerebrosidase, Gaucher disease, Parkinson's disease, $\alpha$-synuclein, LIMP-2 


\subsection{Introduction:}

The GBA gene encodes the lysosomal enzyme glucocerebrosidase (D-glucosylN-acylspingosine glucohydrolase, E.C. 3.2.1.45). The gene is located on chromosome 1q21 and is also known as GBA1. Glucocerebrosidase (GCase) is responsible for the metabolism of glucocerebroside (also known as glucosylceramide) to glucose and ceramide. Deficiency of this enzyme leads to an accumulation' of glucocerebroside in the cells of the reticulo-endothelial system. Homozygous and compound heterozygous $G B A$ mutations cause Gaucher disease (GD) - an autosomal recessive lysosomal storage disorder (Hruska, LaMarca et al. 2008).

Gaucher disease is the most commonly encountered lipidosis, and the most commonly inherited disorder, among the Ashkenazi Jewish population. Globally, the incidence of non-neuronopathic GD is approximately 1:60 000. In the Ashkenazi Jewish community, the incidence peaks at 1:850-1:950 (Cassinerio, Graziadei et al. 2014). More than 300 unique mutations have been found spanning across the whole $G B A$ gene. Five common mutations; namely p.N370S, p.L444P, c.84GGIns, IVS2+1 G>A, and Rec NciI - the recombination between the GBA gene and the pseudogene - are responsible for $70-98 \%$ of all GD cases in different populations (Sato, Morgan et al. 2005, Hruska, LaMarca et al. 2008). The accumulation of glucocerebroside in the cells of the reticuloendothelial system leads to manifestations in numerous organ systems - including the liver, spleen, bone marrow, lungs, and nervous system. There are both non-neuronopathic (type 1) and neuronopathic forms of GD (types 2 and 3). GD has a large range of phenotypes, with varying degrees of severity. Type 1 GD varies from asymptomatic adults to adolescents with markedly enlarged abdomens or crippling bone disease. It accounts for approximately $90 \%$ of all GD cases. Type 2 GD is associated with children who succumb to the neurological disease in infancy. Type 3 GD resembles type 1 GD, with additional neurological symptoms (Hruska, LaMarca et al. 2008, Cassinerio, Graziadei et al. 2014).

Since there is so much variety in the GD phenotype, other factors may play an important role in the presentation of the disease. GCase is synthesized in the endoplasmic reticulum (ER)-bound polyribosomes, from where it is translocated to the ER. After N-linked glycosylations, GCase is transported to the Golgi apparatus and then trafficked to the lysosomes. Mutant GCase variants have varying levels of retention by the ER and are more prone to undergo ER-associated degradation in the proteasomes. The degree of ER retention and proteosomal degradation influences the severity of the GD phenotype (Ron and Horowitz 2005). Some GBA mutations do not result in a loss of GCase 
activity (Bae, Yang et al. 2015).

Parkinson's disease (PD) is the second most common neurodegenerative disease, after Alzheimer's disease. The death of dopaminergic neurons in the substantia nigra pars compacta is the central pathological feature of PD. The motor symptoms - resting tremor, rigidity and bradykinesia form the traditional PD triad (Dauer and Przedborski 2003). However, non-motor symptoms like depression, constipation, pain, sleep disorders; genitourinary problems, cognitive decline and olfactory dysfunction are becoming increasingly well recognized. The non-motor symptoms may precede the motor symptoms by many years (Chaudhuri, Healy et al. 2006). The presence of Lewy bodies and Lewy neurites in brain regions affected by PD are defining neuropathological hallmarks. Lewy bodies- and neurites mainly consist of filamentous $\alpha$-synuclein, encoded by the SNCA gene. They are also central to the pathology of dementia with Lewy bodies (Spillantini, Crowther et al. 1998).

In 2004, it was observed that first-degree relatives of GD patients had a much higher incidence of Parkinson's disease (PD) in comparison to the normal population (Goker-Alpan, Schiffmann et al. 2004). In 2009, an analysis of 5691 PD participants and 4898 controls from 16 centers confirmed the association between GBA mutations and Parkinson's disease. Both GD patients and heterozygous $G B A$ mutation carriers are at an increased risk to develop PD (Sidransky, Nalls et al. 2009). In industrialized countries, the prevalence of PD is generally estimated at $0.3 \%$ of the entire population. This increases with age to $1-2 \%$ in persons over 60 years and $3-4 \%$ in those over 80 . Standardized incidence rates estimate a $1.5 \%$ life-time risk to develop PD (Corti, Lesage et al. 2011). Data from a large Gaucher Registry show that the probability for type 1 GD patients to develop PD is $5 \%-7 \%$ before age 70 and $9 \%-12 \%$ before age 80 , while the $G B A$ mutations occur in $5-10 \%$ of PD patients. This makes $G B A$ mutations the most common genetic risk factor for PD found to date (Rosenbloom, Balwani et al. 2011, Schapira and Gegg 2013). The risk for PD is influenced by the severity of the mutation. Carriers of severely pathogenic GBA mutations (c.84GGIns, IVS2+1 G>A, p.V394L, p.D409H, p.L444P and RecTL) have an odds-ratio of 9.92-21.29 to develop PD, whilst carriers of mild GBA mutations (p.N370S, p.R496H) have an odds-ratio of 2.84-4.94 (Gan-Or, Giladi et al. 2008, Gan-Or, Amshalom et al. 2015). The p.E326K mutation has been identified as a PD risk factor through a large-scale meta-analysis, but it is not considered to be severe enough to cause GD (Pankratz, Beecham et al. 2012, Duran, Mencacci et al. 2013). 
Investigations into the GCase activity in different brain regions of both sporadic and GBA-PD participants show a decrease of GCase activity in the substantia nigra, cerebellum and caudate regions (Gegg, Burke et al. 2012, Chiasserini, Paciotti et al. 2015). Similar changes were observed in dementia with Lewy bodies (Chiasserini, Paciotti et al. 2015), and during normal aging. Reports on GCase reduction in the frontal cortex of sporadic and GBA-PD patients have been conflicting. This is likely due to differences in PD staging and the accumulation of $\alpha$-synuclein in the frontal cortex at the time of examination (Murphy, Gysbers et al. 2014). In healthy persons, GCase activity in the substantia nigra and putamen gradually diminishes. With time, the GCase activity of healthy can be reduced to levels comparable with $G B A$ mutation carriers. This age-dependent reduction in GCase activity may lower the threshold to develop PD in persons with- and without GBA mutations (Rocha, Smith et al. 2015). However, GCase only accounts for $15 \%$ of total brain glucocerebroside metabolism. The remaining glucocerebroside is metabolised by GCase2. GCase2 is a distinct non-lysosomal glucocerebrosidase encoded by the GBA2 gene on chromosome 9p13.3. GCase2 associates with the ER and the Golgi-apparatus. GCase2 is up-regulated in the brains GD mice and leukocytes from GD patients. This may explain why not all patients with GD develop neurological disease. Whilst the activity of GCase2 is dependent on GCase, the reverse does not apply (Burke, Rahim et al. 2013, Körschen, Yildiz et al. 2013, Schöndorf, Aureli et al. 2014). In a mouse model, deleting the GBA2 gene reduces some of the peripheral symptoms of GD (Mistry, Liu et al. 2014). The efficacy of $G B A 2$ deletion or reduction on neuropathology remains unknown. However, it is unlikely to become a therapeutic target for $G B A$-PD, since GBA2 mutations are also linked to neurological diseases like hereditary spastic paraplegia and cerebellar ataxia (Vitner, Vardi et al. 2015).

There are many theories that link GCase loss-of-function to the pathological processes in PD. Mazzulli, Xu et al. (2011), observed that loss of GCase activity in neuronal cultures causes $\alpha$-synuclein accumulation and oligomerization. This causes neurotoxicity through aggregate-dependent mechanisms. In turn, elevated $\alpha$-synuclein inhibits the lysosomal maturation and activity of normal GCase. $\alpha$-Synuclein hinders GCase transport from the endoplasmic reticulum to the lysosome. GCase and $\alpha$-synuclein form a self-propagating bidirectional positive feedback loop, which continues over time until the threshold for neurodegeneration is reached. In transgenic GD mice, $\alpha$-synuclein and ubiquitin aggregates accumulate in numerous brain regions (cortex, hippocampus, basal ganglia, brainstem, and some cerebellar regions). GCase also accumulates in these regions and the progression of aggregates coincides with neurological 
manifestations. Overt neurological symptoms do not develop in all GD mouse strains. This reflects the clinical situation, where the majority of GD patients will never develop PD (Xu, Sun et al. 2011). Improvement of GCase function reduces $\alpha$-synuclein aggregation in several animal models of synucleinopathy regardless of the $G B A$ mutation status. Improving GCase function may be an important strategy for both idiopathic and $G B A$-associated PD (Sardi, Clarke et al. 2013). Recently, there have been a large number of studies investigating the role of GCase in the pathology of PD. This review summarizes current knowledge of the cell biology and pathology of $\mathrm{PD}$ in relation to $G B A$.

\subsection{Molecular functions of $G B A$}

\subsubsection{The link between GCase function and $\alpha$-synuclein accu- mulation:}

Several recent publications have hypothesized an association between reduced GCase activity and increased $\alpha$-synuclein levels in both in vitro and in vivo models. In PD, deposition of $\alpha$-synuclein occurs widely in the central and peripheral nervous system. In theory, PD progression should be associated with the spreading of $\alpha$-synuclein aggregates between neurons. Neuronal cell lines, that overexpress $\alpha$-synuclein, release exosomes that contain $\alpha$-synuclein. These exosomes can transfect normal neurons. Uptake of external aggregated $\alpha$-synuclein leads to co-aggregation of the external and endogenous $\alpha$-synuclein. These co-aggregates are expelled from the neuron, and in turn, transfects other neurons nearby with $\alpha$-synuclein aggregates. Lysosomal dysfunction and depletion of GCase activity promote the propagation of $\alpha$-synuclein aggregates (Alvarez-Erviti, Seow et al. 2011, Bae, Yang et al. 2014). A correlated mutation analysis of all vertebrate species shows that GCase and $\alpha$-synuclein likely co-evolved. Mutations in either protein may disrupt a beneficial interaction between them (Gruschus 2015).

The interaction between GCase and $\alpha$-synuclein depends on the cellular location and the $\mathrm{pH}$. In vitro studies show that membrane-bound $\alpha$-synuclein but not unbound forms - interacts with GCase to form a complex that reduces GCase function. This complex selectively forms at the lysosomal $\mathrm{pH}$. The $\alpha$-synuclein-GCase interaction has a larger $\alpha$-synuclein region on the membrane surface than in solution. The $\alpha$-helix of bound- $\alpha$-synuclein acts as a mixed inhibitor of GCase, with an $\mathrm{IC}_{50}$ value in the micro molar range. Normally GCase binds to the membrane lipid bilayer where it partially inserts itself. The GCase active site likely lies just above the membrane-water interface. Upon interaction with membrane-bound $\alpha$-synuclein, GCase is displaced away from 
the membrane. This could impede substrate access and disturb the GCase active site. In return, GCase moves the helical residues of bound $\alpha$-synuclein away from the bilayer. This could negatively impact the lysosomal degradation of $\alpha$-synuclein. Normally GCase requires saposin C as a co-factor. Saposin C competes with $\alpha$-synuclein for binding to the GCase active site, thus preventing some GCase inhibition (Yap, Gruschus et al. 2013, Yap, Velayati et al. 2013, Gruschus, Jiang et al. 2015, Yap, Jiang et al. 2015).

The link between GCase and $\alpha$-synuclein accumulation has been convincingly demonstrated in studies that show brain regions with $\alpha$-synuclein accumulation have reduced GCase activity. This correlation has also been demonstrated in the periphery, with reduced leukocyte GCase activity linked to increased plasma levels of $\alpha$-synuclein oligomers (Nuzhnyi, Emelyanov et al. 2015). Mutations in both the $G B A$ gene and the $S N C A$ gene (which encodes $\alpha$-synuclein) may compound the PD phenotype. In a transgenic study, mice expressing GBA L444P together with wild-type human SNCA had a $40 \%$ reduction in GCase activity - which triggered the $\alpha$-synuclein accumulation in cortical neurons. When the L444P mutation was co-expressed with the SNCA A53T mutation, the mice showed exacerbated motor and gastrointestinal symptoms in comparison to mice with only the A53T mutation (Fishbein, Kuo et al. 2014).

A mere reduction in GCase activity may not be enough to cause $\alpha$-synuclein accumulation and neuron death. Dermentzaki, Dimitriou et al. (2013) treated neuronal cell cultures with conduritol- $\beta$-epoxide $(\mathrm{C} \beta \mathrm{E})$, a pharmacological inhibitor of GCase, for 7 days. No changes in $\alpha$-synuclein levels, clearance or formation of $\alpha$-synuclein oligomers were evident after the therapy. There was also no significant impairment of the lysosomal machinery, despite severe inhibition of GCase function. Murphy, Gysbers et al. (2014) observed that a reduction in brain GCase activity occurs in the early stages of sporadic PD. Thereafter GCase activity remains stable as the PD progresses. GBA protein levels and activity is reduced in brain regions with $\alpha$-synuclein accumulation. Although the lysosome dysfunction is significant in these regions, it is not enough to cause neuronal cell death. Ceramide and general chaperone-mediated autophagy are also reduced in affected brain regions. The authors suggest that $G B A$ deficits contribute to the neuropathology through altering the lysosomal membrane properties and autophagy, rather than through glucocerebroside accumulation. Gegg, Sweet et al. (2015) also observed that GBA mutations do not increase the accumulation of glucocerebroside in brain regions with low $\alpha$-synuclein pathology. In vitro experiments show that pharmacologically reducing the synthesis of glucocerebroside - thus reducing substrate accumulation - does not 
attenuate $\alpha$-synuclein toxicity, whilst reducing GCase activity increases the damaging effects of $\alpha$-synuclein. The reduction of enzymatic activity, rather than the accumulation of the substrate appear to be the neurotoxic event (Noelker, Lu et al. 2015). During aging, the phosphorylation of $\alpha$-synuclein at serine 129 increases. This increases its propensity to aggregate. In addition to reduced GCase activity, aged primates also have lower Protein Phosphatase A2 (PPA2) activity in their striatum and hippocampus than in their cerebellum and occipital cortex. PPA2 normally dephosphorylates $\alpha$-synuclein. Reduced PPA2/GCase activity correlates with increased oligomerization of $\alpha$-synuclein. This may underlie regional vulnerability to neurodegeneration (Liu, Chen et al. 2015). In Drosophila, GBA-knockdown causes the accumulation of proteinase K (PK) resistant $\alpha$-synuclein. The increased in PK-resistant $\alpha$-synuclein - rather than increased amounts of total $\alpha$-synuclein - worsened locomotor dysfunction, loss of dopaminergic neurons and retinal dysfunction. This study suggests that the damaging effects of GCase dysfunction may be due to accelerated $\alpha$-synuclein misfolding (Suzuki, Fujikake et al. 2015).

Recently, two new fish models shed light on the GCase- $\alpha$-synuclein cascade. The zebrafish genome does not contain $S N C A$. A novel zebrafish line (Danio Rerio), with a homozygous 23bp deletion in the $G B A$ ortologue, showed accumulation of glucocerebroside and Gaucher cells in the brain. This was accompanied by up-regulation of microRNA-155 and activation of microglia both associated with inflammation. Hexosaminidase and chitotriosidase, both established GD biomarkers, were markedly abnormal and the mitochondrial respiratory chain activity was impaired. Motor activity became impaired around 12 weeks, at which time ubiquitin-positive intraneuronal inclusions reduced the number of dopaminergic neurons (Keatinge, Bui et al. 2015, Keatinge, DaCosta et al. 2015). These fish were not more susceptible to 1-methyl-4phenylpyridinium $(\mathrm{MPP}+$ ) toxicity than the wild-type (Payne, Keatinge et al. 2015). Uemura, Koike et al. (2015) discovered that Japanese rice fish (Oryzias latipes) homozygous for $G B A$-missense variants can survive for months without any GCase activity. The fish had demonstrated similar neuropathology to human type 2 GD and accumulation of $\alpha$-synuclein in the neuronal axons, but this was not lethal. Surprisingly, additional genetic knockout of $\alpha$-synuclein on top of the $G B A$ knockout did not increase the lifespan. Axonal pathology and dopaminergic or noradrenergic neuronal losses were also not attenuated by $\alpha$-synuclein knockout. This suggests that there are other factors needed for the neurotoxic cascade.

Another study has identified the Alzheimer's disease (AD) proteins as pos- 
sible players in type 2 GD neuropathology. AD is caused by the accumulation of $\beta$-amyloid and tau proteins into insoluble clumps termed neurofibrillary tangles. $\beta$-amyloid is formed by splicing of the amyloid precursor protein (APP). Whether the accumulation of $\beta$-amyloid is the cause or consequence of AD is currently under debate (Drachman 2014). In PD dementia, Alzheimer's pathology frequently co-occurs with Lewy bodies (Compta, Parkkinen et al. 2014). $\beta$-amyloid may indirectly promote the fibrillation of $\alpha$-synuclein. Transgenic type 2 GD mice have significant $\beta$-amyloid and APP in their cortex, striatum, hippocampus, and substantia nigra. APP aggregates in neurons, where it co-localizes with $\alpha$-synuclein (Xu, Xu et al. 2014). Conversely, a deficiency of APP can increase the expression of glucosylceramide synthase and increase glucocerebroside levels (Grimm, Hundsdörfer et al. 2014). There is likely to be a common degenerative pathway involving many of the classical neurodegenerative players in neuronal GD. These observations may also be relevant for the milder $G B A$-associated PD.

The majority of APP / $\alpha$-synuclein accumulate in the region of the mitochondria where it leads to morphological changes and likely functional impairment of the mitochondria. Smaller fractions of APP/ $\alpha$-synuclein co-localize with markers for the autophagosome system and the lysosomal associated membrane protein 1 (LAMP-1) - a marker for lysosomes (Xu, Xu et al. 2014). Together, LAMP-1 and LAMP-2 contribute approximately $50 \%$ of all the proteins in the lysosome membrane and thus serve as a marker for the lysosome compartment (Eskelinen 2006). The observation that APP/ $\alpha$-synuclein aggregates are more prevalent in the mitochondria than in the autophagosomes and lysosomes, support the hypothesis that the degradation of these proteins is reduced in the brains of neuronal Gaucher disease mice (Xu, Xu et al. 2014). In idiopathic PD, reduced LAMP-1 (and thus reduced lysosomes) is observed in nigral neurons $\alpha$-synuclein accumulation (Chu, Dodiya et al. 2009). In contrast, the amount and size of LAMP-1 particles are increased in midbrain neurons derived from patients with GD or GBA-PD via induced pluripotent stem cell technology. In these neurons, the autophagosome marker light chain 3 is also increased. However, co-localization between LAMP-1 and light chain 3 protein is reduced suggesting impaired autophagosome-lysosome fusion in $G B A$-mutant neurons (Schöndorf, Aureli et al. 2014). 


\subsubsection{Neuro-inflammation and ROS:}

Neuronal inflammation and the formation of reactive oxygen species (ROS) contributes to the death of dopaminergic neurons. McNeill, Magalhaes et al. (2014) studied fibroblasts from participants with GD, GBA heterozygotes with/without PD and controls. The GCase activity and protein levels were reduced in the experimental groups in comparison to controls (Activity: GD= $5 \%, G B A-\mathrm{PD}=59 \%, G B A$-no $\mathrm{PD}=56 \%$, Protein levels: $\mathrm{GD}=58 \%, G B A-\mathrm{PD}=$ $41 \%, G B A$-no-PD $=32 \%$ ). This lead to an increase in ROS across all groups $(\mathrm{GD}=62 \%, G B A-\mathrm{PD}=68 \%, G B A$-no- $\mathrm{PD}=70 \%$ increase $)$. Cellular glutathione levels were reduced in the experimental groups. The transcript levels of the antioxidant NQ01 were elevated in both GD and $G B A$-heterozygotes with/without $\mathrm{PD}$ as a possible compensatory mechanism. Thus, GCase insufficiency increases neuronal oxidative stress. Increasing glutathione levels can have antioxidanteffects. This can be achieved by $N$-acetylcysteine administration (Holmay, Terpstra et al. 2013).

Chemotactic factors are a key component of the neuro-inflammatory cascade. These factors recruit immunologic mediators to the site of inflammation. In a model of type $1 \mathrm{GD}$, the activation of Gaucher cells initiates the inflammatory cascade. This is followed by the release of various chemokines and cytokines. Chemokine (C-X-C) Motif Ligand (CXCL)-9, CXCL-10 and CXCL11 recruit T-lymphocytes, CXCL-13 recruits B-lymphocytes and CXCL-1 and CXCL-2 recruit polymorphonuclear neutrophils. Monocytes are recruited by chemokine (C-C) motive ligand (CCL)-1, CCL-5 and monocyte chemotactic protein-5. Granulocyte macrophage colony stimulating factor and granulocyte colony stimulating factor cause the mobilization and growth of immune cells (T-lymphocytes, B-lymphocytes, polymorphonuclear neutrophils, and monocytes). The monocytes exit the circulation and migrate to various tissues where they mature into macrophages and dendritic cells. Excess glucocerebroside is accumulated in the macrophages and dendritic cells. These cells release additional cytokines and chemokines, which restart the cascade. The elevated chemokines promote the migration of immune cells from the peripheral blood and bone marrow into tissues, like the brain. This contributes to the neuro-inflammation process (Pandey, Jabre et al. 2014). GBA-PD patients have higher plasma levels of monocyte-associated inflammatory mediators (interleukin 8, monocyte chemotactic protein and macrophage inflammatory protein) than idiopathic cases (Chahine, Qiang et al. 2013). Gaucher cells also release more pro-inflammatory kinase p38 and interleukin 6 in response to an inflammatory stimulus (Kitatani, Wada et al. 2015). Activated microglia and astrocytes are detected prior to neuronal death in the brain regions affected by 
GD. The level of inflammatory mediators correlates with the disease severity. A robust neuro-immune response of astrocytosis and activation of microglia occurs in rodent nigral neurons with reduced GCase and $\alpha$-synuclein pathology (Ginns, Mak et al. 2014). Increased complement protein C1q, also contributes to the neuropathology (Rocha, Smith et al. 2015).

\subsubsection{Metal deregulation:}

The accumulation of trace element metal ions contributes to $G B A$-associated neurotoxicity. $\mathrm{Ca}^{2+}$ is important for the transmission of depolarizing signals in neurons. Dopaminergic neurons in the substantia nigra have innate autonomous activity, which is sustained by L-type $\mathrm{Ca}^{2+}$ channels. Sustained $\mathrm{Ca}^{2+}$ entry creates metabolic stress in these neurons which can be aggravated by genetic and environmental factors (Surmeier, Guzman et al. 2010). Fibroblasts from persons with type $1 \mathrm{GD}$ or PD have more $\mathrm{ER} \mathrm{Ca}^{2+}$ release than asymptomatic $G B A$-mutation carriers. The increased release was partly due to aging. $G B A-\mathrm{PD}$ fibroblasts also have reduced lysosomal $\mathrm{Ca}^{2+}$ stores, which can be ascribed to accelerated remodelling of the $\mathrm{Ca}^{2+}$ stores (Kilpatrick, Magalhaes et al. 2015). Schöndorf, Aureli et al. (2014), derived inducible pluripotent stem cells (iPSCs) from participants with GBA-PD and GD - which they differentiated into midbrain dopaminergic neurons. The neurons with $G B A$ mutations have increased neuronal calcium-binding protein 2. This disrupts calcium homeostasis, which increases vulnerability to cytosolic calcium stress responses. A correction of the $G B A$ mutation rescued the iPSC pathological phenotype.

Iron is elevated in the substantia nigra of PD participants. Normally, excess iron can be sequestrated in the form of ferritin or neuromelanin. Alternatively, the excess iron is deposited in the brain during normal aging. Excess iron levels lead to neuronal death through ROS or reactive nitrogen species (SianHülsmann, Mandel et al. 2011). GD is characterized by increased iron in Gaucher cells. Approximately $60 \%$ of type 1 GD participants have increased serum ferritin levels. The storage of glucocerebroside in the macrophages causes an inflammatory response with deregulation of iron recycling and the release of cytokines. The cytokines influence the production of hepcidin - a regulator of iron homeostasis. Increased hepcidin levels inhibit iron recycling in the macrophages and thus cause iron retention in the macrophages. Traditional substrate reduction therapy does not alter serum ferritin or hepcidin levels. However, treatment of type 1 GD patients with iron chelators (deferoxamine or deferasirox) for four months or longer, markedly decreased the serum ferritin 
(from $1028 \mathrm{ng} / \mathrm{ml}$ to $445.8 \mathrm{ng} / \mathrm{ml}$ ) and hepcidin (from $339.7 \mathrm{ng} / \mathrm{ml}$ to 147.8 $\mathrm{ng} / \mathrm{ml}$ ) levels without reducing pro-inflammatory cytokines. In these patients, the levels of serum ferritin remained low 1 year after the last chelation therapy (Medrano-Engay, Irun et al. 2014).

Disturbances in zinc homeostasis can cause neurodegeneration through protein misfolding and oxidative stress. Zinc is also increased in the substantia nigra of persons with PD (Kozlowski, Luczkowski et al. 2012). Mutations in the ATP13A2 gene (PARK9) are associated with juvenile-onset Parkinsonism. ATP13A2 encodes a zinc pump, which transports zinc into membrane-bound components or vesicles. Overexpression of the ATP13A2 protein confers resistance to extracellular zinc $\left(\mathrm{Zn}^{2+}\right)$ and promotes the transport of $\alpha$-synuclein to the exosomes. Cells with ATP13A2 knockdown are more sensitive to extracellular $\mathrm{Zn}^{2+}$ (Kong, Chan et al. 2014, Tsunemi, Hamada et al. 2014). Primary neurons with silenced ATP13A2 have decreased lysosomal sequestration of $\mathrm{Zn}^{2+}$ and increased expression of $\mathrm{Zn}^{2+}$ transporters. The $\mathrm{Zn}^{2+}$ increases lead to lysosome dysfunction which contributes to the $\alpha$-synuclein-GCase pathological cascade. Either zinc chelation or improvement of ATP13A2 function can improve the phenotype (Tsunemi and Krainc 2014).

\subsubsection{Glucocerebrosidase and LIMP-2:}

The lysosomal integral membrane protein type-2 (LIMP-2) is an abundant protein of the lysosomal membrane. LIMP-2 is a mannose-6-phosphate (M6P) independent lysosomal transport receptor for GCase (Reczek, Schwake et al. 2007). Loss of LIMP-2 increases lipid storage and causes functional disturbances in the autophagy/lysosome system. It also reduces lysosomal GCase activity in a mouse model. Ultimately, the LIMP-2 dysfunction cascade leads to accumulation of $\alpha$-synuclein in dopaminergic neurons (Rothaug, Zunke et al. 2014). LIMP-2 binds to GCase and releases it via a pH-dependent histidine trigger. LIMP-2 can localize the ceramide portion of glucocerebroside adjacent to the GCase catalytic cells. LIMP-2 also plays a role in M6P-dependent sorting of GCase to the lysosome. The N325 residue of LIMP-2 is covalently linked to a sugar (Man9GlcNAc2) with a terminal M6P. The LIMP-2 terminal $\mathrm{M} 6 \mathrm{P}$ has a similar affinity for the M6P receptor than for GCase (Zhao, Ren et al. 2014).

A unique 11 amino-acid sequence on GCase is responsible for LIMP-2dependent targeting. The interactions between GCase and LIMP-2 are heavily influenced by GCase asparagine-399, isoleucine-402 and isoleucine-403. If any of 
these amino acids undergo substitution with alanine, the binding to LIMP-2 is decreased. This increases the secretion of GCase and reduces trafficking to the lysosome through altering the $\mathrm{pH}$-dependent binding of GCase (Liou, Haffey et al. 2014). LIMP-2 is down-regulated by the microRNA miR-127-5p, which in turn reduces GCase activity and protein levels (Siebert, Westbroek et al. 2014). Gonçalves, D'Almeida et al. (2014), observed that mouse-embryonic fibroblasts with LIMP-2 deficiency were more susceptible to infection by the protozoa Trypanosoma cruzi. When the fibroblasts were treated with GCase enzyme replacement therapy, the level of susceptibility to trypanosomal infection was reduced to similar levels of wild-type fibroblasts. Improving GCase function may also improve LIMP-2 function.

The link between LIMP-2 and GBA-PD is inconclusive. In human fibroblasts, the $G B A$-mutation status does not correlate with LIMP-2 expression (McNeill, Magalhaes et al. 2014). Typically, GCase deficiency in the brains of sporadic PD patients is not caused by reduced LIMP-2 expression (Gegg, Burke et al. 2012, Murphy, Gysbers et al. 2014). LIMP-2 is encoded by the SCARB2 gene. Recently, two intronic $S C A R B 2$ polymorphisms were investigated in PD association studies. An association between rs6825004 and rs6812193 and PD was identified in Greek and North-American/ European descent cohorts, respectively. The association between rs6812193 and PD was confirmed in a German and Austrian population, but not replicated in a Han Chinese cohort. However, neither polymorphism appears to affect the levels of LIMP-2 protein or RNA expression (Hopfner, Schulte et al. 2013, Maniwang, Tayebi et al. 2013). SCARB2 mutations are also implicated in inherited forms of myoclonic epilepsy. Myoclonic epilepsy also occurs as part of the spectrum of neurological involvement of GD, especially when both $G B A$ and $S C A R B 2$ mutations are present (Velayati, DePaolo et al. 2011).

\subsubsection{Other molecules implicated in GCase pathology:}

GCase interacts with a number of molecules involved in synaptic function and plasticity. Reduced GCase function diminishes the plasma levels of brain derived neurotropic factor (BDNF), which can be reversed by enzyme replacement therapy (Vairo, Sperb-Ludwig et al. 2015). BDNF is a neurotropin which plays an important role in neuronal survival and synaptic plasticity (Arancio and Chao 2007). During the normal synaptic function, vesicles containing neurotransmitters are released by the SNARE (N-ethylamide sensitive fusion attachment protein receptor) proteins. Abnormal pre-synaptic $\alpha$-synuclein accumulation impairs synaptic vesicle release (and thus neurotransmitter release) 
through interfering with SNARE proteins. $\alpha$-Synuclein accumulation reduces the vesicle recycling rate at the presynaptic membrane and ultimately a number of vesicles (Garcia-Reitböck, Anichtchik et al. 2010). Neurotransmitter release is reduced in the striatum of murine GD models (transgenic and $\mathrm{C} \beta \mathrm{E}$-induced). This is accompanied by dysfunction of the synapses in the substantia nigra. $\mathrm{C} \beta \mathrm{E}$-treatment reduces dopamine release in response potassium chloride stimuli in nigral dopaminergic neurons. This treatment reduces the size of synaptic vesicles, without altering the mean amount of vesicles (Ginns, Mak et al. 2014). GCase dysfunction also alters microRNAs linked to immune response and synaptic plasticity. MicroRNAs are a class of small non-coding RNAs, which regulate the transcription of proteins from the mRNA code. $\mathrm{C} \beta \mathrm{E}$-therapy increases microRNA-29 and microRNA-142 and reduce microRNA-let7b in the ventral mesencephalon of mice (Ginns, Mak et al. 2014). Conversely, GCase activity and protein levels can be increased by more than $40 \%$ by the microRNAs, MiR-16-5p, and miR-195-5p (Siebert, Westbroek et al. 2014). An analysis of the global profile of microRNA and mRNA expression, in the brains of type 2 GD mice, show that the RNAs involved in inflammation, mitochondrial dysfunction, axonal guidance and synaptic transmission are differentially expressed. The expression of the majority of the microRNAs and mRNAs could be normalized with chaperone therapy (Dasgupta, Xu et al. 2015).

Parkin is an E3 ubiquitin ligase that causes familial forms of PD. Parkin mediates the K48-dependent proteosomal degradation of mutant GCase, but not of normal GCase. Mutations in Parkin impairs its interaction with mutant GCase (Ron, Rapaport et al. 2010). Mutant GCase associates with Parkin to undergo Parkin-mediated degradation. This leads to competition with other Parkin substrates (i.e. PARIS and ARTS) for the available enzyme, resulting in substrate accumulation. The substrate accumulation contributes to neuronal death (Bendikov-Bar, Rapaport et al. 2014). Accumulation of PARIS (Parkin interacting substrate/ Zinc finger protein 746) in the cytoplasm down-regulates genes associated with mitochondrial biogenesis. PARIS represses the transcription of the peroxisome proliferator-activated receptor gamma (PPAR $\gamma$ ) co-activator 1- $\alpha$. PARIS also suppresses the transcription of ribosomal DNA through interactions with components of the RNA polymerase I complex and the Myb-binding protein 1- $\alpha$. These mechanisms contribute to the death of dopaminergic neurons (Kang and Shin 2015). ARTS (apoptosis related protein in TGF $\beta$ signalling pathway) is normally located in the outer mitochondrial membrane, but upon apoptotic stimuli, it translocates to the cytoplasm. In the cytoplasm, ARTS leads to cell death through a pathway involving caspase 
activation. Mutant GCase causes an accumulation of ARTS in the cytoplasm which increases the cleavage of caspase 3 and 9 in response to apoptotic stimuli (Bendikov-Bar, Rapaport et al. 2014).

Three new proteins were recently discovered in neuronal GD. Glycoprotein non-metastatic B (GPNMB) is a trans-membrane protein expressed in melanocytes, osteoclasts, macrophages, neurons, and astrocytes. GPNMB is elevated in the brain tissue of patients with type 2 and type 3 GD. It is also elevated in the cerebrospinal fluid (CSF) of these patients. In GD mice, the amount of CSF GPNMB correlates with the amount of neuropathology. In humans, the CSF GPNMB levels in the correlate with lower scores on cognitive tests. More research into the function of this target in GD is needed, but it may be a promising biomarker of neurological involvement in GD (Zigdon, Savidor et al. 2015). The Receptor Interacting Protein Kinases 1 and 3 (RIPK1 and RIPK3) are serine-threonine kinases that are essential for programmed necrosis (necroptosis). Whether cells undergo apoptosis or necroptosis is determined by caspase 8 . Both RIPK1 and RIPK3 are elevated in the brains of neuronal GD mice, and increased RIPK1 has been confirmed in a case of human GD. Mice with deficient RIPK3 are resistant to $\mathrm{C} \beta \mathrm{E}$ toxicity. These mice have an increased life-span and delayed onset of neuroinflammation (Vitner, Salomon et al. 2014). RIPK1 and RIPK3 inhibition could be promising targets for the management of $G B A$-associated neuropathology. However, there are not currently inhibitors for these targets that cross the blood-brain barrier. Investigating targets up- and downstream of these kinases may yield future therapeutic options (Vitner, Vardi et al. 2015).

\subsubsection{Treatment strategies for GD and $G B A-\mathrm{PD}$ :}

\subsubsection{Peripheral management of GD:}

\section{Enzyme replacement therapy:}

Currently, intravenous enzyme replacement therapy (ERT) and oral smallmolecule substrate reduction therapy (SRT) are the only FDA approved treatment options for patients with non-neuronopathic GD (Shemesh, Deroma et al. 2015). ERT started with the approval of alglucerase in 1991. Imiglucerase, a recombinant GCase, became available in 1994. It is currently the golden standard therapy for the visceral symptoms of GD. 10 year follow up shows that imiglucerase (or alglucerase) therapy improves haemoglobin levels, platelet counts, liver and spleen volume and bone crisis (Weinreb, Goldblatt et al. 2013). 
Newer recombinant ERT drugs include velaglucerase alfa (approved in 2010) and taliglucerase alfa (approved in 2012) (Zimran and Elstein 2014). All the ERT therapies have similar therapeutic efficacy on haematological parameters (Shemesh, Deroma et al. 2015). ERT does not cross the blood-brain barrier at all and is thus inefficient for the neurological manifestations of type 2 and 3 GD (Lachmann 2011).

\section{Substrate reduction therapy:}

SRT aims to reduce glucocerebroside through preventing its biosynthesis by glucosylceramide synthase. Miglustat (N-butyl-1-deoxynojirimycin), a weak inhibitor of glucosylceramide synthase, received approval for the treatment of type 1 GD in 2002 (Lachmann 2003). Gastrointestinal intolerance and tremor are common adverse effects, which reduce patient compliance (Machaczka, Hast et al. 2012). Miglustat crosses the blood brain barrier. However, a recent phase II trial did not observe significant benefit for the neurological manifestations of type 3 GD (Schiffmann, FitzGibbon et al. 2008). Miglustat can reduce the toxicity of subchronic 1-methyl-4-phenyl-1,2,3,6-tetrahydropyridine (MPTP) exposure in an animal model. MPTP is a mitochondrial toxin, which can induce Parkinsonism. However, miglustat is ineffective against $\alpha$-synuclein-induced toxicity (Noelker, Lu et al. 2015). A second SRT drug, Eliglustat, received FDA approval for type 1 GD in 2014. Eliglustat is a strong inhibitor of glucocylceramide synthase (Poole 2014). Eliglustat does not cross the blood-brain barrier. There are efforts to design SRT drugs that are centrally available (Larsen, Wilson et al. 2012).

\subsubsection{Therapeutics for neuronal GD and $G B A-P D$ :}

The newest therapeutic strategy is the small-molecule chaperones. These molecules improve GCase activity through binding to GCase, correcting the folding of mutant GCase in the endoplasmic reticulum and facilitating its trafficking to the lysosomes. Once in the lysosome, the low $\mathrm{pH}$ causes dissociation of the GCase-chaperone complex. Then the residual activity of the mutant GCase hydrolyzes glucocerebroside. After promising preclinical and in vitro work, clinical trials have commenced on molecular chaperones for GD (Parenti, Andria et al. 2015).

Ambroxol is a mixed-type inhibitor of GCase that acts in a pH-dependent manner. It was originally marketed as an expectorant (Maegawa, Tropak et al. 2009). Ambroxol improves GCase activity, along with the amount and mass 
of lysosomes in Gaucher disease fibroblast lines (Bendikov-Bar, Maor et al. 2013, McNeill, Magalhaes et al. 2014). In humans, ambroxol has successfully completed a pilot study in Israel for type 1 GD (Zimran, Altarescu et al. 2013). Chaperones for any lysosomal storage disorder should ideally exhibit maximal binding at the neutral $\mathrm{pH}$ of the ER and little binding at the acidic $\mathrm{pH}$ of lysosomes. Ambroxol's inhibitory activity is maximal at neutral $\mathrm{pH}$ in the $\mathrm{ER}$ and undetectable at the acidic $\mathrm{pH}$ of lysosomes. It stabilizes amino acid segments 243-249, 310-312, and 386-400 near the GCase active site. Ambroxol also interacts with non-active site residues (Maegawa, Tropak et al. 2009). Ambroxol's benefit is limited to mutants that produce a foldable protein with some residual intrinsic enzymatic activity. The chaperoning efficacy of ambroxol varies for common variants. p.G202R, p.N370S, and p.F213I affect the early folding of GCase. Additionally, the variants have distinct subcellular locations. p.N370S GCase exhibits weak lysosomal localization whilst p.G202R GCase is retained in the ER. These mutations respond well to ambroxol therapy ( $\mathrm{Yu}$, Sawkar et al. 2007, Maegawa, Tropak et al. 2009). The L444P mutation is commonly associated with neuronopathic GD. This mutation results in disrupted folding in the ER and impaired post-ER trafficking, which causes it to undergo extensive degradation in the ER (Bendikov-Bar, Ron et al. 2011). Ambroxol does not increase the GCase activity of L444P homozygotes (Maegawa, Tropak et al. 2009), but it can assist with its removal from the ER (Bendikov-Bar, Ron et al. 2011). L444P is located in a non-catalytic domain of GCase, which makes the protein particularly refractory to the rescuing action of active-site directed pharmacological chaperones (de la Mata, Cotán et al. 2015).

In the McNeill, Magalhaes et al. (2014) study, ambroxol increased the GCase activity and LIMP-2 levels in the fibroblasts from GD, GBA-PD, GBA without PD and control participants. This was accompanied by an approximately $50 \%$ reduction of ROS production across all groups. The effect of ambroxol on LIMP-2 was replicated by Ambrosi, Ghezzi et al. (2015), who also reported ambroxol therapy increases the levels of saposin $\mathrm{C}$ and has no effect on Parkin. This confirms that the beneficial effect of ambroxol is mediated through lysosomal mechanisms. Part of this therapeutic benefit can also be ascribed to a 2.25 fold increase in the transcript levels of the transcription factor EB (TF-EB). TF-EB up-regulates the transcription of genes in the Coordinated Lysosomal Expression And Regulation (CLEAR) network in a synchronized fashion. The CLEAR network consists of more than 400 genes involved in lysosomal function, including GBA (McNeill, Magalhaes et al. 2014). Ambroxol shows promise for general lysosomal improvement beyond pure GCase enhancement. It is a promising as a mono-therapy, but also as 
an adjuvant for SRT. The efficacy of SRT is enhanced in vitro by increasing TF-EB expression. However, enhancement of TF-EB activity by itself does not adequately restore lysosomal activity (Awad, Sarkar et al. 2015).

For the L444P genotype, novel bicyclic L-idonojirimycin derivatives are under development (Alfonso, Andreu et al. 2014). $N$-[N'-(4-adamantan-1ylcarboxamidobutyl)thiocarbamoyl]-1,6-anhydro-L-idonojirimycin (NAdBT$\mathrm{AIJ})$, in combination with co-enzyme Q10, shows in vitro promise to relieve mitochondrial dysfunction and increase GCase activity in refractory GCase genotypes (de la Mata, Cotán et al. 2015). However, idonojirimycin-derivatives and other newer chemical classes of pharmacological chaperones have not yet undergone preclinical development and are not viable therapeutic options at this stage.

Isofagomine is another established chaperone, which acts as an active-site inhibitor of GCase. Isofagomine increases the amount of GCase which reaches the lysosome (Steet, Chung et al. 2006). Isofagomine progressed to phase II clinical trials for GD (Amicus Therapeutics, Cranberry, NJ) but it did not meet clinical end-points and was abandoned. A recent study investigated whether isofagomine is beneficial in mice with synucleopathies. The study used thy1$\alpha$-synuclein mice - which overexpress human $\alpha$-synuclein with normal GCase function. These mice have motor deficits; including problems with speed, balance and coordination; and olfactory dysfunction. Treatment with isofagomine for four months increased the stabilization, trafficking, and activity of GCase in both thy1- $\alpha$-synuclein and wild-type mice. Of note, different therapeutic effects were observed with different isofagomine regimens. 3 days on treatment, 4 days off regimen was more effective at improving olfactory function, whilst 7 days on - 7 days off regimen was more effective at reversing motor deficits. This may due be to differing increases in GCase activity in different brain regions after each regimen. Isofagomine reduced the levels of diffuse $\alpha$-synuclein in the cytoplasm of the nigral dopaminergic neurons. However, it increased the nigral surface area covered with insoluble $\alpha$-synuclein aggregates. Isofagomine may increase the size of the insoluble $\alpha$-synuclein aggregates without altering global $\alpha$-synuclein levels. Isofagomine also reduced the activated microglial pattern in the test group, and thus reduced neuronal inflammation. Pharmacological chaperone therapy may be beneficial for participants with synucleopathies, irrespective of their GBA mutation status (Richter, Fleming et al. 2014).

Mutant GCase is more susceptible to degradation by pathways involving Parkin, Heat Shock protein (Hsp) 70, Hsp90 $\beta$ and the endoplasmic reticulum 
associated pathway. Modulating these pathways is another therapeutic strategy (Yang, Swallows et al. 2014). Normally, misfolded GCase is recognized by Hsp90 $\beta$, which guides it through the valosin-containing protein-associated degrading pathway. The histone deacetylase inhibitors (HDACis) interfere with the acetylation of histone proteins in DNA. The HDACis also hyperacetylate the middle domain of Hsp90 $\beta$, which impairs its ability to recognize mutant GCase. This reduces the degradation of mutant GCase and thus increases its activity (Yang, Rahimpour et al. 2013). The heat-shock protein chaperone complex (consisting of Hsp90, Cdc37, and Hsp90-Hsp70-organizing protein) associates with mutant GCase. The complex recruits Hsp27, which assists with the targeting of misfolded GCase to degradation pathways. Direct inhibition of Hsp27 increases GCase activity (Yang, Swallows et al. 2014, Yang, Wang et al. 2015). Celastrol is a small molecule chaperone that prevents GCase degradation. Celastrol interferes with the establishment of the heat-shock protein chaperone complex and reduces Hsp90-associated protein degradation. Further celastrol increases the expression of the Bcl2-associated athanogene 3 (BAG3), DnaJ homolog subfamily B members 1 and 9 (DNAJB1/9) and heat shock $70 \mathrm{kDa}$ proteins $1 \mathrm{~A}$ and $1 \mathrm{~B}$. The BAG family molecular chaperone regulator 3 improves its folding and catalytic activity of GCase. Targeting the molecular chaperones may be an alternative therapeutic strategy for GD (Yang, Swallows et al. 2014).

McNeill, Magalhaes et al. (2014) studied the co-localization of GCase with the ER-marker calnexin. In control cells and $G B A$-heterozygotes with or without PD, the GCase accumulated in punctate structures at the cell periphery, which morphologically resembled lysosomes and it did not co-localize with calnexin. However, in GD fibroblasts most of the GCase accumulated peri-nuclear and co-localized with the calnexin and the peripheral GCase was reduced. This indicated that in controls and heterozygous $G B A$ carriers, most of the GCase is transported to the lysosomes, while in GD GCase concentrates in the endoplasmic reticulum. Tan, Genereux et al. (2014) discovered that depleting ERdj3, an ER resident Hsp40, redirects mutant GCase to the pro-folding calnexin associated pathway in the ER. This increases the folding, trafficking and functioning of mutant GCase. Depleting ERdj3 also reduces the rate of the degradation of mutant GCase in participant derived fibroblasts. Simultaneous reduction in ERdj3-mediated degradation of mutant GCase together with enhancement of the calnexin folding pathway could restore the function of mutant GCase in Gaucher disease.

The transfer of a functional gene with a vector is another strategy to increase GCase activity. In rodent models of mutant $\alpha$-synuclein-induced 
neurodegeneration, overexpression of the $G B A$ gene with an adeno-associated virus vector into the brain reduces $\alpha$-synuclein accumulation (Rocha, Smith et al. 2015). Delivery of $G B A$ with a lentiviral system controlled by cellular promoters reverses the peripheral symptoms in type 1 GD mice (Dahl, Doyle et al. 2015). Peripheral gene correction has not been effective in human clinical trials. For use in neuronal GD and $G B A-P D$, the gene therapy needs to reach the brain. Injections directly into the brain are not an acceptable option in most therapeutic scenarios (Vitner, Vardi et al. 2015). In neonatal and very young animals, intravenous adeno-associated virus therapy may cross the blood-brain barrier (Rahim, Wong et al. 2011). At this stage, gene-therapy is not a viable therapeutic option, but it may become available in the future.

\subsubsection{New cellular models for studying GCase:}

Aflaki, Stubblefield et al. (2014) generated and characterized macrophages from GD participants. They also created iPSC-derived macrophages from fibroblast cell lines derived from participants with type 1 or type 2 GD. Both macrophage models had reduced GCase activity and increased lysosomal storage of glucocerebroside and glucocylsphingosine. The macrophage models also demonstrated efficient phagocytosis of bacteria, reduced intracellular ROS production, and reduced chemotaxis. The disease phenotype was reversed with small molecule chaperone treatment. Woodard, Campos et al. (2014), studied iPSC-derived midbrain dopaminergic neurons from identical twins, with the N370S mutation, that were discordant for PD. The dopaminergic neurons from both twins had an approximately 50\% reduction in GCase activity and an approximately three-fold increase in $\alpha$-synuclein protein levels. The neurons also had a reduced capacity to synthesize and release dopamine. However, the affected twin's neurons had even lower levels of dopamine coupled with increased expression of the monoamine-oxidase B (MAO-B) enzyme and impaired intrinsic level activity. The MAO-B enzyme degrades dopamine and other biological amines. MAO-B inhibition is a therapeutic target in PD. Treatment of the neuron with MAO-B inhibitors together with overexpressing wild-type $G B A$, through a viral vector, normalized the $\alpha$-synuclein and dopamine levels. This combination therapy could be beneficial for $G B A$-PD patients. Sun, Florer et al. (2015), converted type 2 GD and unaffected fibroblasts into neurons via IPSC and neuronal precursor cell intermediates. All of the cell types had similar levels of GCase deficiency. Patch-clamp electrophysiological studies of derived neurons indicated a lower resting membrane potential in the type 2 GD cells. The amplitudes of action potentials, sodium and potassium currents were also reduced. Similar observations were made in control cells treated with 
$\mathrm{C} \beta \mathrm{E}$. Reduced neuronal excitability is important in the context of neuronal dysfunction. Participant derived macrophages, iPSC-derived macrophages and iPSC-derived dopaminergic neurons could all be promising models to study the $G B A$-associated pathology and the efficacy of therapies.

\subsubsection{Brain ceramide profiles and other lysosomal enzymes in Parkinson's disease:}

GCase produces ceramide through the breakdown of glucocerebroside. This pathway is also known as the salvage pathway. It represents the most energy efficient way of ceramide synthesis in post-mitotic cells, where it may account for 50-90\% of all sphingolipid production (Tettamanti, Bassi et al. 2003). In lysates of the putamen and cerebellum, of sporadic-and GBA-PD patients, there does not appear to be more total GCase substrate accumulation - glucocerebroside or glucosylsphingosine (deacetylated glucocerebroside) - than in controls. However, a trend of increased GM2 and GM3 gangliosides was observed. The GM3 ganglioside is a complex lipid that lies biosynthetically upstream of glucocerebroside (Gegg, Sweet et al. 2015). Fibroblasts and derived IPSCs from GD and GBA-PD patients mainly contain GM3 gangliosides, with small amounts of other ganglioside types (GM1, GM2, GD1a, GD3). Differentiation into midbrain dopaminergic neurons and neuronal enrichment reduce the proportions of GM3 and GD3, in relation to total gangliosides. Ratios of GM1, GD1a, GD1b, and GT1b in cultured neurons resemble normal human brain distribution. The activity of the non-lysosomal glucocerebrosidase $(G B A 2)$ and $\beta$-galactosidase are increased in fibroblasts with GD mutations but reduced in the derived neurons (Schöndorf, Aureli et al. 2014). The influence of $G B A$-mutations on $\beta$-hexosaminisase activity is debated, with McNeill, Magalhaes et al. (2014) observing an increased activity in GBA-PD fibroblasts, which was not replicated by Schöndorf, Aureli et al. (2014) in fibroblasts or Chiasserini, Paciotti et al. (2015) in brain tissue.

Abbott, Li et al. (2014), investigated the effect of PD on ceramide composition in the anterior cingulate cortex (which is significantly affected by Lewy body pathology) and the occipital cortex (which is normally spared in $\mathrm{PD})$. They observed that in anterior cingulate cortex of PD participants, but not occipital cortex, the total ceramide (53\% lower) and sphingomyelin ( $42 \%$ lower) levels were reduced. In PD participants, ceramide species in the anterior singular cortex shift towards shorter acyl chain composition and ceramide synthase I increase. Post-mortem analysis of the primary motor cortex of Lewy Body Dementia (DLB) patients, with GBA mutations, showed changes 
in sphingolipid composition. DLB is discussed later in this review. In these patients, the total sphingomyelin concentrations were slightly decreased, but total ceramides and dihydrosphingomyelin species were increased. Glucosylceramide was enriched - albeit not statistically significant - in $G B A$-carriers. Of note, GM3 was highly enriched accompanied by a significant accumulation of galactosylceramide (Clark, Chan et al. 2015).

Plasma ceramide levels and monohexosylceramide metabolism is also altered in PD participants without $G B A$ mutations. Although ceramide levels are decreased in brain regions affected by PD, increases are observed in peripheral blood plasma. Higher plasma ceramide levels are associated with worse cognition. Increased plasma ceramide and monohexylceramide species vary in carbon chain length from $\mathrm{C} 16$ to $\mathrm{C} 24$. Other lipids, like cholesterol and triglycerides, have similar plasma levels in PD patients and controls. PD is thus specific for increased ceramide levels (Mielke, Maetzler et al. 2013). Increased blood serum levels of C18:1 hexosylceramide, C18 ceramide, and sphingosine may be useful as a bio-marker to distinguish $G B A-\mathrm{PD}$ from idiopathic PD (Glickman, Bodamer et al. 2015). The CSF may also contain useful biomarkers for GBA-PD. GBA-PD patients have lower GCase 1, GCase 2, $\alpha$-mannosidase and $\beta$-mannosidase activity than idiopathic PD patients. $G B A$-PD patients also have lower GCase 1, GCase 2 and $\beta$-galactosidase activity than controls. These changes do not occur in idiopathic PD (Schöndorf, Aureli et al. 2014). The overall amounts of fatty acids in the CSF also differ between GBA-PD and idiopathic PD. GBA-PD patients have significantly lower levels of palmitoleic, oleic, linoleic, arachidonic, eicosapentaenoic and decosahexaenoic acids, as well as less total fatty acids, in their CSF. Abnormalities in fatty acid metabolism may be specific to GBA-PD (Schmid, Schleicher et al. 2012). 


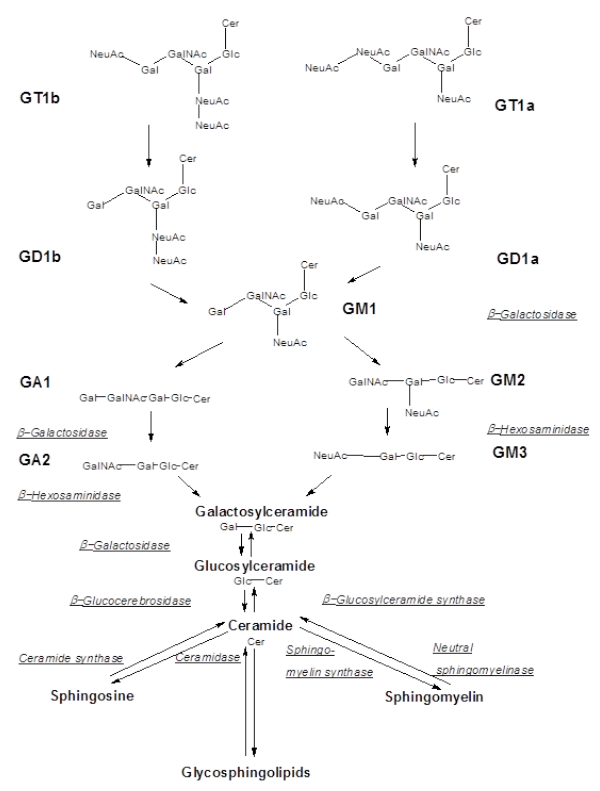

Figure 2.1: Pathways of the altered ceramide metabolites (adapted from Tettamanti, Bassi et al. (2003)).

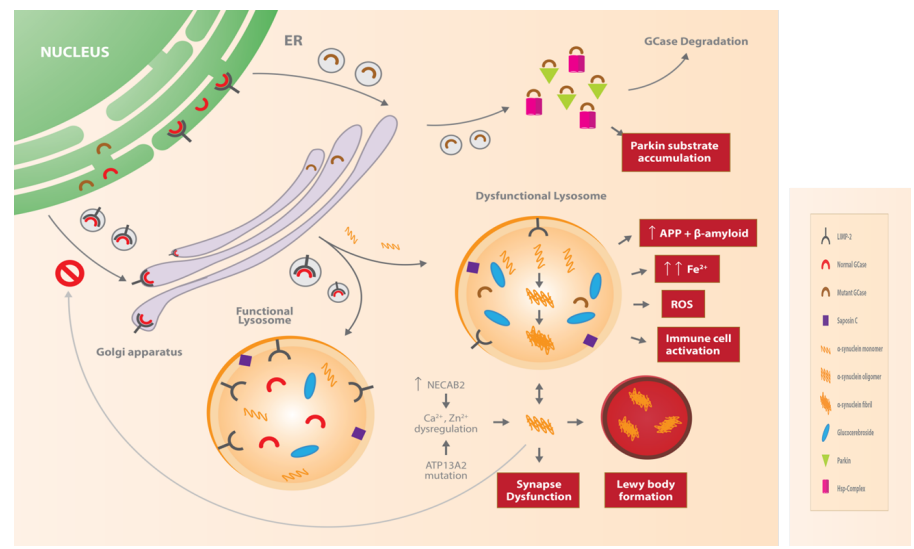

Figure 2.2: An overview of the $G B A-\mathrm{PD}$ neuropathological cascade 


\subsection{Clinical pictures for $G B A-P D$ :}

\subsubsection{Brain imaging abnormalities:}

Various imaging studies have looked for differences between $G B A-\mathrm{PD}$ and other types of PD. Dopamine transporter imaging scans (123I-FP-CIT DaTSCANs) show that the asymmetry in radio ligand uptake in $\mathrm{PD}$ associated with $G B A$ or LRRK2 mutations is greater than in $\mathrm{PD}$ associated with mutations in other genes (SNCA, PINK1 or Parkin). The asymmetry could reflect that mono-allelic GBA and $L R R K 2$ mutations initially have focal neurodegeneration due to interactions with additional endogenous or exogenous pathogenic factors. These results show that $G B A-\mathrm{PD}$ may be associated with greater dopaminergic neuronal loss than other types of PD (McNeill, Wu et al. 2013). On Magnetic Resonance Imaging (MRI) scans, GBA-PD participants have less fractional anisotropy in their olfactory tracts, corpus callosum, and anterior limb of the internal capsule bilaterally, as well as in the right anterior external capsule, left cingulum, parahippocampal tract, parietal portion of the superior longitudinal fasciculus, and occipital white matter than controls. GBA-PD participants also have less fractional anisotropy of the majority of the white matter tracts than PD patients without GBA mutations (Agosta, Kostic et al. 2013). Brockmann, Hilker et al. (2012) performed combined Proton $\left({ }^{1} \mathrm{H}\right)$ and Phosphorus $\left({ }^{31} \mathrm{P}\right)$ Magnetic Resonance Spectroscopic Imaging (MRSI) quantifying N-acetylaspartate (NAA) (a marker of neuronal integrity), ethanolamine containing components (markers of membrane phospholipid metabolism), highenergy phosphates (energy metabolites) and choline in GBA-PD patients and controls. In the putamen, of the $G B A-\mathrm{PD}$ group, NAA was reduced and glycerophosphoethanolamine was increased. In the midbrain, both NAA and choline were reduced. No changes in energy metabolites were observed. These findings suggest that $G B A-\mathrm{PD}$ is associated with alterations in membrane phospholipid metabolism.

Transcranial sonography and functional imaging of GBA-PD and GDwithout-PD patients revealed an increased bilateral median maximal area of substantia nigral echogenicity and substantia nigral hyper-echogenicity and reduced brainstem raphe echogenicity in comparison to controls. However, there were no significant differences between $G B A-\mathrm{PD}$ or GD and idiopathic PD patients. The hyper-echogenicity of the substantia nigra is thought to reflect abnormal iron accumulation and this was present in GD patients irrespective of whether they developed PD. The nigral hyper-echogenicity in GD patients correlated with MRI-T2 hypo-intensity of the substantia nigra pars compacta. Positron emission tomography scans of $G B A-\mathrm{PD}$ participants did not find alterations in comparison to idiopathic PD (Barrett, Hagenah et al. 2013, 
Böttcher, Rolfs et al. 2013, Kresojević, Mijajlović et al. 2013). $\mathrm{F}^{18}$-dopa PET scans show similar striatal dopamine loss patterns among patients with both GD and PD (GD-PD) and idiopathic PD patients indicating similar levels of mid-brain dopaminergic neuron loss. The dopamine loss was greater in the caudal striatum, with less reduction in the caudate. Resting regional cerebral blood flow analysis with $\mathrm{H}_{2}{ }^{15} \mathrm{O}$ PET could distinguish between GD-PD and idiopathic PD patients. GD-PD patients showed decreased regional cerebral blood flow in the lateral parieto-occipital association cortex and precuneus bilaterally. This decrease in cerebral blood flow had a pattern suggestive of diffuse Lewy body disease and parallels the increased frequency of cognitive complaints reported by patients with $G B A$-associated parkinsonism (GokerAlpan, Masdeu et al. 2012).

\subsubsection{Clinical presentation:}

$G B A$ mutations are associated with a spectrum of Parkinsonian phenotypes ranging from Parkinson disease, mostly of the akinetic type, to a less common phenotype characteristic of Lewy body dementia. The levodopa responsiveness ranges from refractive to favorable responses with dyskinesia and wearing-off complications developing over time. Motor assessments show that asymmetric tremor and bradykinesia are the most common symptoms at onset for $G B A-\mathrm{PD}$ patients. Postural instability and gait abnormalities are also common (GokerAlpan, Lopez et al. 2008, Wang, Cai et al. 2014). In general, PD patients have slightly lower peripheral blood GCase activity than controls $(11.53 \mathrm{~mol} / \mathrm{l} / \mathrm{h}$ versus $12.11 \mu \mathrm{mol} / \mathrm{l} / \mathrm{h})$. The average values of the test were $0.85 \mu \mathrm{mol} / \mathrm{l} / \mathrm{h}$ for $G B A$ homozygotes/compound heterozygotes with $\mathrm{PD}, 7.88 \mu \mathrm{mol} / \mathrm{l} / \mathrm{h}$ for $G B A$ heterozygotes with $\mathrm{PD}$ and $11.53 \mu \mathrm{mol} / \mathrm{l} / \mathrm{h}$ for PD patients without $G B A$ mutations (Alcalay, Levy et al. 2015).

\subsubsection{Influence of $G B A$ mutations on age-of-onset of dis- ease:}

Alcalay, Dinur et al. (2014) investigated the age specific risk for PD in participants with GD disease. They enrolled 427 participants with type 1 GD, 697 heterozygous GBA mutation carriers, and 154 control participants. All participants were of Ashkenazi Jewish descent and 98\% of GD participants had at least 1 N370S mutation. Among the participants that developed PD, participants with GD had a younger age-of-onset (mean 54.2 years) than $G B A$ heterozygous carriers (mean 65.2 years). The estimated age-specific risk for $\mathrm{PD}$ at 60 years of age was $4.7 \%$ for GD participants, $1.5 \%$ for $G B A$-carriers 
and $0.7 \%$ for controls. At age 80 , the risk of $\mathrm{PD}$ increased to $9.1 \%$ for GD participants, $7.7 \%$ for $G B A$-carriers and $2.1 \%$ for controls. They conclude that the number of mutant $G B A$ alleles may influence the age-of-onset of PD but both GD participants and $G B A$-carrier participants had a similar risk by age 80 . Wang, Cai et al. (2014) studied the clinical profile of Chinese PD participants with the L444P GBA mutation; p.G2385R or p.R1682P mutations in the leucine repeat-rich kinase 2 ( $L R R K 2)$ gene or idiopathic PD. The $L R R K 2$ gene has been implicated in both familial and sporadic PD cases. The LRRK2-PD participants clinically resembled idiopathic PD participants. However, the GBA-PD participants had a significantly younger age-of-onset of $\mathrm{PD}(51.45 \pm 9.14$ years vs. $58.41 \pm 11.03$ years $)$. This may be mutation dependent. Alcalay, Levy et al. (2015) observed significantly higher peripheral blood GCase activity in carriers of the LRRK2 p.G2019S mutation than in persons without LRRK2 mutations ( $13.7 \mathrm{micromol} / \mathrm{l} / \mathrm{h}$ versus 11.93 micromol $/ \mathrm{l} / \mathrm{h}$ ) which may reflect a distinct disease mechanism in these patients.

Kumar, Ramirez et al. (2013) observed that patients carrying a severe heterozygous GBA mutation (p.L444P, p.D409H, p.H255Q, p.R463C, RecNcil) had a significantly earlier age-of-onset $(45.1 \pm 8.32$ years) than patients with mild heterozygous (p.N370S) mutations (56.17 \pm 7.63 years). A large-scale pooled analysis showed smaller variation with an age-of-onset of $53.1 \pm 11.2$ years for carriers of severe mutations (c.84GGIns, IVS2+1 G>A, p.V394L, p.L444P, RecTL) and $58.1 \pm 10.6$ years for mild mutations (p.N370S and p.R496H) (Gan-Or, Amshalom et al. 2015) Additional genetic susceptibility factors may modify the age-of-onset of disease. The minor allele of SNP in the bridging integrator 1 (BIN1) gene region (rs13403026) was associated with an increase in age-of-onset of $10.7 \pm 6.8$ years for patients with mild $G B A$ mutations and $17.1 \pm 2.5$ years in patients with severe GBA mutations (Gan-Or, Amshalom et al. 2015). BIN1 is the second most important risk locus for late-onset Alzheimer's disease. It primarily mediates tau pathology but also has roles in endocytosis, inflammation, calcium homeostasis and apoptosis (Tan, Yu et al. 2013).

Li, Sekine et al. (2014) studied the prevalence of $G B A$ mutations in Japanese familial PD cases to draw conclusions about the clinicogenetic profile. $21.5 \%$ of their families with PD were heterozygous carriers of $G B A$ mutations compared to $1 \%$ of controls. GBA mutations occurred in $25.9 \%$ of families with autosomal dominant $\mathrm{PD}$ and in $8.5 \%$ of families with autosomal recessive PD. The age of onset of PD was similar in both groups (49.1 \pm 11.7 for GBA$\mathrm{PD}, 51.3 \pm 14.5$ for non- $G B A \mathrm{PD})$. The link to familial $\mathrm{PD}$ is notable since 
$G B A$ mutations have largely been found in cases of sporadic PD. The authors conclude that while $G B A$ mutations are a strong and common risk factor for $\mathrm{PD}$, it may not be a definitive causal gene in familial PD. Liu and Zhang (2014), performed a meta-analysis on $G B A-P D$ non-familial case-control studies performed up to 2012. The association between PD and $G B A$ mutations was confirmed in a total group analysis (fixed OR: 4.825, random OR: 4.791) and in different subpopulations analysed. They found that the evidence of the $G B A-\mathrm{PD}$ link was not contributed just by one study and that there was no publication bias. The authors concluded that a significant association exists between $G B A$-mutation status and non-familial PD.

\subsubsection{GBA in cognitive impairment and depression:}

Persons with GD and heterozygous $G B A$ mutation carriers, with no prior history of PD, scored significantly lower on cognitive assessments (Montreal Cognitive Assessment and the Mini Mental State Exam) than controls (McNeill, Duran et al. 2012). Persons with GBA-PD have decreased white matter in the inter-hemispheric, frontal corticocortical, and parahippocampal tracts in comparison to idiopathic PD patients. These white-matter abnormalities correlate with verbal fluency scores in $\mathrm{PD}$, thus reflecting a greater tendency for cognitive impairment among GBA mutation carriers (Agosta, Kostic et al. 2013). A long-term follow-up study ( $\mathrm{n}=121$, median follow-up for 71 months) showed that persons with $G B A$-PD had a significantly increased hazard ratio to progress to develop dementia $(5.7, \mathrm{p}=0.003)$ and progress to Hoehn and Yahr stage $3 \mathrm{PD}(4.2, \mathrm{p}=0.003)$ in comparison to idiopathic PD. GBA mutations may influence natural progression of PD (Winder-Rhodes, Evans et al. 2013). Conversely, in a 2-year follow-up study on $G B A$-mutation positive patients without $\mathrm{PD}$ at enrolment, initial mild cognitive impairment was the main predictor of a decline in motor function at the study endpoint (Beavan, McNeill et al. 2015).

Brockmann, Srulijes et al. (2015) observed a younger age-of-onset, a more rapid progression of motor- and cognitive decline and increased mortality in patients with $G B A$-associated PD. Their cohort consisted of $20 G B A-\mathrm{PD}$ and 27 idiopathic PD participants, therefore their findings may be limited by a small sample size. Malec-Litwinowicz, Rudzińska et al. (2014) investigated the clinical features of $G B A-\mathrm{PD}$ in $138 \mathrm{PD}$ participants from Poland. In their study, p.N370S and p.T369M accounted for all the identified $G B A$ mutations. In their study, the p.N370S mutation acted as a significant risk factor for cognitive impairment (60\% risk in p.N370S carriers vs. 19.6\% risk in idiopathic $\mathrm{PD}$ group). The mean age-of-onset of PD was the same in both groups. Zokaei, 
McNeill et al. (2014) found that there are deficits in the visual short-term memory precision in $G B A$ mutation carriers with/without PD. The deficits were also present in PD participants without $G B A$ mutations. The visual memory deficits in $G B A$-carriers without PD were attributed to a systemic increase in the interference from the features of other items stored (misbinding errors). The deficits in PD participants were explained by increased random responses. Individuals who had both $G B A$ mutations and PD suffered from both types of error and had the worst performance. Drug utilization studies indicate that $G B A$-PD patients are more likely to take long term acetylcholine esterase inhibitors than idiopathic PD patients (Barrett, Shanker et al. 2014).

\subsubsection{Depression and neuropsychiatric symptoms:}

Depression, anxiety, social dysfunctions, hallucinations and other psychosis are more frequently reported in GBA-PD cases than in idiopathic PD (GokerAlpan, Lopez et al. 2008, Barrett, Shanker et al. 2014, Li, Sekine et al. 2014, Wang, Cai et al. 2014, Beavan, McNeill et al. 2015). Quetiapine therapy appears to be well-tolerated for the treatment of hallucinations in GBA-PD (Barrett, Shanker et al. 2014).

\subsubsection{Sleep disorders:}

Rapid-eye-movement (REM) sleep behaviour disorder (RBD) is a parasomnia characterised by dream-enacting behaviours related to unpleasant dreams and loss of muscle atonia during REM sleep. RBD is a common complaint of patients with Parkinson's disease. RBD often precedes the development of a Lewy body disorder like PD or DLB or mild cognitive impairment and it is thus a selective prodromal marker for PD (Boot, Boeve et al. 2012, Iranzo, Tolosa et al. 2013, Schenck, Boeve et al. 2013). Initial RBD screening of GD patients $(\mathrm{n}=30), G B A$ heterozygotes $(\mathrm{n}=30)$ and controls $(\mathrm{n}=30)$ without $\mathrm{PD}$, failed to find a meaningful increase in the frequency of RBD in $G B A$-positive individuals. However, a two-year follow up showed significant worsening of the $G B A$-positive group on the RBD questionnaire. The amount of RBD individuals was higher in the $G B A$-positive groups than in controls. However, this did not reach statistical significance (McNeill, Duran et al. 2012, Beavan, McNeill et al. 2015). Insomnia and associated daytime sleepiness and fatigue are common disabling symptoms in PD. Insomnia and fatigue correlate with a more advanced disease state as well as depression, anxiety, cardiovascular, urinary and thermoregulatory dysfunction (Metta, Logishetty et al. 2011, Kurtis, Rodriguez-Blazquez et al. 2013). Long-acting dopaminergic 
stimulation improves subjective sleep quality. Eszopiclone and quetiapine may be used for insomnia in $\mathrm{PD}$, whilst clonazepam and melatonin can alleviate RBD. Caution is advised with the use of all the sedating medications in PD (Diederich and McIntyre 2012). Fatigue is also a common complaint in GD, where it may be related to anaemia and other haematological abnormalities (Balwani, Fuerstman et al. 2010, Zion and Rosenbaum 2015). Fatigue in GD is normally not resolved by enzyme replacement therapy. Acupuncture may be beneficial in these patients (Samuels, Elstein et al. 2012).

\subsubsection{Olfactory dysfunction:}

Olfactory dysfunction is considered to be a prodromal non-motor symptom of PD. A reduced performance on olfactory tests (like the University of Pennsylvania Smell Identification Test) was noted in both GD patients and $G B A$-heterozygotes with and without PD across numerous studies (GokerAlpan, Lopez et al. 2008, McNeill, Duran et al. 2012, Beavan, McNeill et al. 2015).

\subsubsection{Autonomic system dysfunction:}

Dysautonomia including constipation, orthostatic hypotension, bladder dysfunction and erectile dysfunction have been reported in GBA-PD patients. However, in the majority of studies, general autonomic dysfunction is not statistically significantly increased in the $G B A$-PD subgroup or in $G B A$-mutation carriers without PD (Goker-Alpan, Lopez et al. 2008, McNeill, Duran et al. 2012, Li, Sekine et al. 2014). Wang, Cai et al. (2014) observed increased sweating, sexual dysfunction, and constipation in the $G B A-\mathrm{PD}$ group.

\subsubsection{Ocular signs:}

GD often involves the visual system. Oculomotor deficits are among the earliest deficits identified in neuronopathic (type 3) GD patients. The most common manifestation is markedly slow horizontal saccades are often called "saccadic initiation failure" or "ocular motor apraxia" or "horizontal supranuclear gaze palsy". Saccades are the quick simultaneous movement of both eyes between two phases of fixation in the same direction. This is used to scan the immediate surroundings. In type $3 \mathrm{GD}$, the vertical saccades are also slower than in controls with downward saccades progressively more affected than upward saccades. The ratio between vertical saccade peak duration and amplitude slope significantly correlated with IQ and hand eye-coordination. 
Pupillary light responses are also reduced in type 3 GD (Benko, Ries et al. 2011, Narita, Shirai et al. 2014). Additionally, pre-retinal and intra-retinal white spots and accumulation of retinal Gaucher cells have been observed in GD cases (Seidova, Kotliar et al. 2009).

In $\mathrm{PD}$, impaired initiation of memory guided saccades (without a visual stimulus) and hypometric saccadic movements have been observed across all saccadic parameters. The visually guided saccades are affected to a smaller extent. PD patients also have a reduced ability to suppress unwanted saccades. All of these abnormalities are linked to basal ganglia dysfunction and can be altered by levodopa therapy and deep brain stimulation (See the review by Terao, Fukuda et al. (2013)). It has recently been suggested that dysfunctional eye movements in PD are associated with reduced cortical executive function rather than being related to the ponto-cerebellar circuits or the oculomotor brainstem nuclei (Gorges, Müller et al. 2015)(Gorges, 2015). Decreased saccadic amplitude in PD is primarily related to motor impairment. Prolonged saccadic latency is related to both motor and cognitive impairment. Increased antisaccade error rates are also associated with reduced cognitive performance and this is already impaired in newly diagnosed PD patients before the onset of medication (MacAskill, Graham et al. 2012, Antoniades, Demeyere et al. 2015).

Other common visual problems in PD include reductions in visual acuity, contrast sensitivity, colour and motion perception as well as alterations in electroretinogram latencies and amplitudes. A reduced thickness of the retinal nerve fibre layer (measured by optical coherence tomography) and glaucomatous-like perimetric defects has been observed by numerous authors in PD, DLB and PD-associated dementia (Inzelberg, Ramirez et al. 2004, Altintaş, Işeri et al. 2008, Moreno-Ramos, Benito-León et al. 2012, Tsironi, Dastiridou et al. 2012). These findings have not been replicated across all studies (Archibald, Clarke et al. 2011). A pilot study by McNeill, Roberti et al. (2013) with GD patients and GBA-carriers without PD identified retinal thinning among the patients who also had premotor signs of Parkinson disease such as hyposmia and memory disturbances, as well as one patient with motor symptoms. Retinal thinning was not observed in patients without premotor symptoms. The authors suggested that optical coherence tomography may be useful in the early identification of patients likely to develop Parkinson disease. Interestingly, a small trial showed that patients with GD only have superior colour discrimination ability in comparison to patients with PD only. Male patients with GD + PD have an intermediate error rate in the colour discrimination test, but they still outperformed patients with only PD. The 
same effect was not seen for $G B A$-heterozygotes (Simon-Tov, Dinur et al. 2015).

\subsection{3 $G B A$ mutations and other forms of Parkinsonism and de- mentia:}

Protein misfolding, accumulation, aggregation and the eventual formation of deposits are common features of many neurodegenerative diseases. In PD, PD dementia, Dementia with Lewy bodies (DLB) and Multiple System Atrophy (MSA), the pathology is caused by aggregated and phosphorylated $\alpha$-synuclein. These disorders are termed synucleinopathies. In Alzheimer's disease (AD), pathology is caused by $\beta$-amyloid and tau accumulation into neurofibrillary tangles. Other tauopathies include Progressive Supranuclear Palsy (PSP) and Corticobasal Degeneration (CBD) - both forms of atypical Parkinsonism. Cooccurrence of synucleinopathies and tauopathies occur frequently in diseases like DLB, PDD and the Lewy body variant of AD (ADLBV) (Moussaud, Jones et al. 2014). GBA seems to be more frequently associated with synucleinopathies. Asselta, Rimoldi et al. (2014) screened an Italian cohort for GBA mutations in exon 9 and 10. Their cohort consisted of participants with primary PD, DLB, PSP, MSA and CBD. GBA mutations were strongly associated with the Lewy body diseases and occurred in $4.5 \%$ of PD participants and $13.8 \%$ of DLB participants vs. $0.63 \%$ of controls. However, GBA mutations had a weak association with other types of Parkinsonism like PSP and CBD. Among the PD group, participants with $G B A$ mutations had an average age of onset of 52 \pm 10 years, which was 5 years younger than the idiopathic PD group. They were also $14 \%$ more likely to have a positive family history of PD. The higher prevalence of $G B A$ mutations in the DLB cohort suggests a more aggressive phenotype.

Nalls, Duran et al. (2013) conducted a multicentre analysis for $G B A$ mutations in patients with Lewy body dementia and noted an odds ratio of 8.28 for $G B A$ mutations in DLB participants and 6.48 in PD dementia participants. $G B A$ mutations reduced the age-of-onset of DLB by five years on average. The Lewy body dementias, DLB and ADLBV, are also significantly associated with variations in the $S M P D 1$ and $M C O L N 1$ genes. SMPD1 encodes Sphingomyelin Phosphodiesterase in the ceramide synthesis pathways. SMPD1 mutations are associated with the lysosomal storage disorder Niemann-Pick disease type A and B. MCOLN1 encodes mucolipin-1, a lysosomal transmembrane protein. MCOLN1 mutations cause mucolipidosis - another lysosomal storage disorder. This suggests that multiple hits in the lysosomal pathway can increase the risk of Lewy body disorders (Clark, Chan et al. 2015). 
The reported frequencies of GBA mutations in the other types of Parkinsonian syndromes are discussed in table 1 . From the reported data, it is evident that $G B A$-mutations are associated with the neuronal synucleopathies (PD, $\mathrm{PD}$ dementia, DLB), but unexpectedly $G B A$-mutations are rare in MSA. In PD and DLB, $\alpha$-synuclein accumulates in neurons, whereas in MSA, $\alpha$-synuclein mainly accumulates in the oligodendroglia (Wenning, Stefanova et al. 2008). $G B A$ mutations may cause regional specific $\alpha$-synuclein accumulation. $G B A$ mutations are nearly absent in the Tauopathies (PSP, CBD). Reports on the prevalence of $G B A$ mutations in $\mathrm{AD}$ are contradictory. Whilst not a Parkinsonian syndrome, essential tremor (ET) is associated with an increased risk to develop PD. GBA mutations occur with a similar frequency in the essential tremor case and control groups and are thus probably not associated with disease (Clark, Kisselev et al. 2010).

\subsubsection{New populations screened for $G B A$ mutations:}

This review will give an overview of novel populations screened and $G B A$ mutations identified since 2014. For an overview of earlier $G B A$-PD frequencies per population group, refer to Sidransky and Lopez (2012). Of particular interest, in the Tabuleiro de Norte community in Brazil, all the GD cases and carriers screened carried a single mutation, p.G377S. This community has the highest GD prevalence rate in Brazil, with 1 in every 4000 persons in the community affected. This mutation was identified in carriers and GD participants in different generations. Together with the history of the community and a genealogy study, Chaves, Pereira et al. (2014) ascribed the high prevalence of GD in this community to a founder effect combined with consanguinity.

Malini, Grossi et al. (2014) identified and characterized the function of 11 novel GBA mutations from Italian GD patients. Two of the mutations were de novo germ-line mutations. This has important implications for genetic counselling in GD. The functional analysis included seven single missense alleles, p.P159S, p.N188I, p.E235K, p.P245T, p.W312S, p.S366R, and p.W381C. The missense mutations resulted in very low or no GCase activity. With the p.P159S mutation, $15 \%$ of the residual enzymatic activity was retained. Two alleles carrying cis mutations (p.N188S; p.G265R) and (p.E326K; p.D380N) were analysed. By itself, the p.N188S mutant retained $25 \%$ of enzymatic activity. The p.E326K mutant retained 54\% enzymatic activity. This is in line with previous reports of p.N188S acting as a modifier variant and p.E326K as a mild variant. The cis combination of the mild alleles with p.G265R and 
Table 2.1: The frequency of $G B A$ mutations in Parkinsonian syndromes and other neurodegenerative diseases.

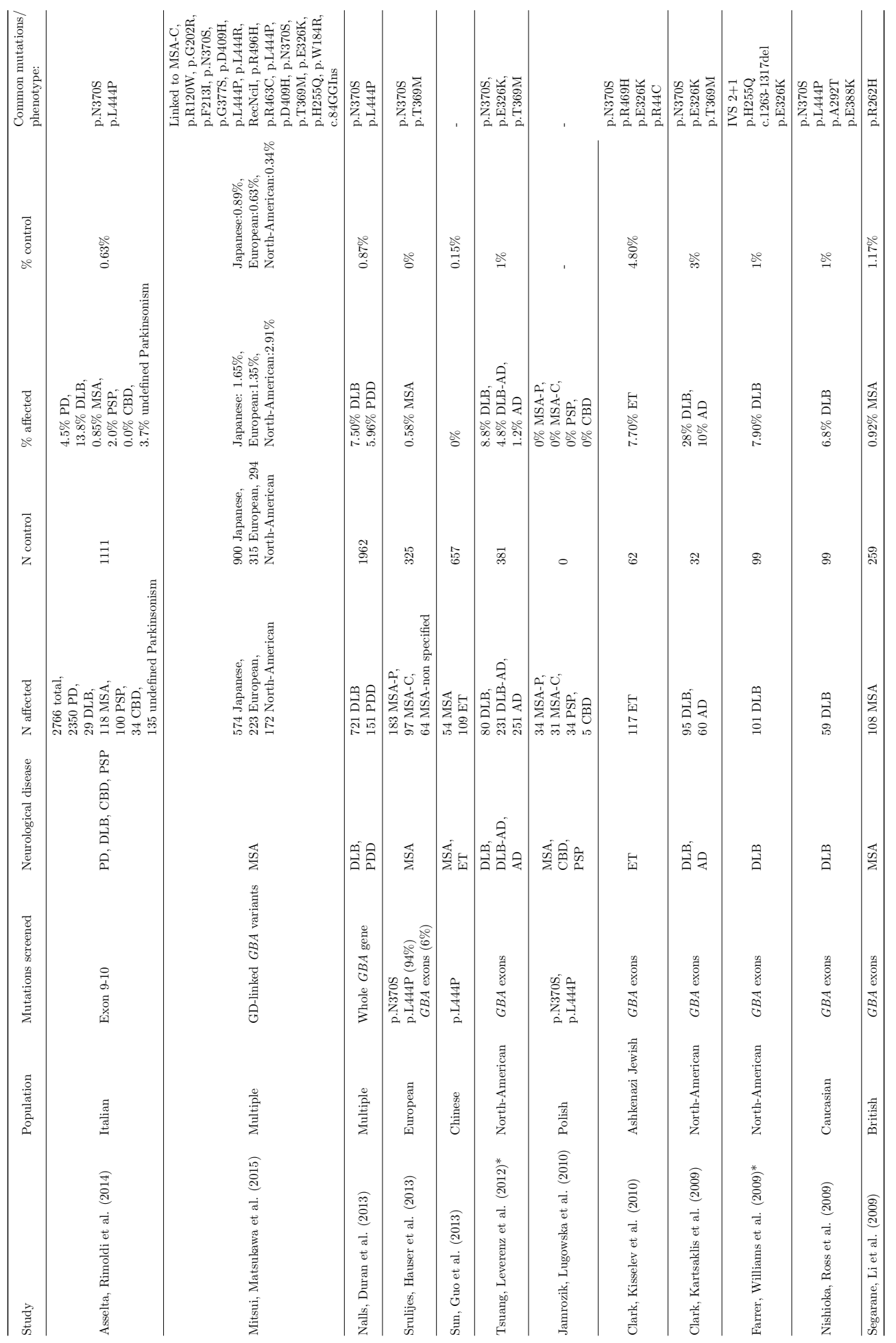


Table 2.2 Gaucher disease populations screened since 2014

\begin{tabular}{|c|c|c|c|c|c|c|c|}
\hline Study & Population & Gaucher disease & Mutations screened & Number of cases & Number of controls & Most common mutations & Novel mutations \\
\hline Ankleshwaria et al. (2014) & Indian & Type 1,3 & GBA exons, exon-intron boundaries & $33 \mathrm{GD}$ & 0 & p.L444P (61\%) & $\begin{array}{l}\text { p.G289A }(3 \%) \\
\text { p.I466S }(3 \%)\end{array}$ \\
\hline Jack et al. (2014) & Canadian & Type 1 & $G B A$ exons, exon-intron boundaries & $2 \mathrm{GD}$ & 0 & p.N370S $(100 \%)$ & $\begin{array}{l}\text { p.C23W } \\
\text { IVS } 7-1 \quad A>G\end{array}$ \\
\hline Machaczka \& Klimkowska (2014) & Iranian & Type 1 & $G B A$ exons, exon-intron boundaries & $1 \mathrm{GD}$ & 20 & - & $\begin{array}{l}\text { p.F266L } \\
\text { p.I347S }\end{array}$ \\
\hline Chaves et al. (2014) & Brazillian & Type 1 & $G B A$ exons, exon-intron boundaries & $\begin{array}{l}50 \mathrm{GD} \\
20 \mathrm{GBA} \text { carrier }\end{array}$ & 111 & p.G377S $(100 \%)$ & - \\
\hline Mattosova et al (2014) & Slovakian & Type $1,2,3$ & $G B A$ exons, exon-intron boundaries & $14 \mathrm{GD}$ & 0 & $\begin{array}{l}\text { p.N370S } \\
\text { p.L444P } \\
\text { p.G377S } \\
\text { p.D409H } \\
\text { RecNcil }\end{array}$ & $\begin{array}{l}\text { p.C4W } \\
\text { p.L96P } \\
\text { p.H311N } \\
\text { c.745delG } \\
\text { c.11127_1128delTT }\end{array}$ \\
\hline
\end{tabular}

p.D380N abolished all enzymatic activity. The common mutation p.N370S retained $38 \%$ enzymatic activity, whilst p.L444P retained $13 \%$. All the novel missense mutations lie in domain III of the GCase protein, which contains the active catalytic site. Apart from p.P159S, p.G304R, and p.E326K, all the mutations are involved in substrate recognition. These 3 mutations are located on the protein surface, where they may interfere with the correct coupling of GCase partners or indirectly with the catalytic activity of other residues. The type of amino acid substitution had a large effect on enzymatic activity. The N188 residue is involved in the correct substrate positioning. The p.N188I mutation resulted in a completely inactive protein. A small asparagine was replaced with a big, hydrophobic isoleucine. Replacing it with the small polar serine (p.N188S) retained a high level of residual activity. Two acceptor splice sites in introns 5 (c.589-1G>A) and 9 (c.1389-1G>A), caused the synthesis of aberrant mRNA. Both intronic variations are predicted to introduce premature stop codons, which resulted in the frame shifts p.I197Vfs*7 and p.L464Sfs*24, respectively. When the novel severe mutations occurred in the compound heterozygous state with p.N370S, type 1 GD was observed. In combination with another severe mutation, type 2 GD presented. The functional analysis showed that altering the catalytic site is deleterious for enzymatic activity. Other novel mutations identified in GD cases are discussed in table 2, whilst table 3 gives an overview of PD populations screened since 2014 .

\subsection{Discussion and conclusion:}

There is a significant amount of evidence that supports the role of the feedforward circuit between $\alpha$-synuclein and GCase in the neuropathology of PD. In PD participants, GCase activity is reduced in brain regions where $\alpha$-synuclein accumulates. Reduced GCase activity promotes the spread of $\alpha$-synuclein aggregates between neurons which advances PD. Membrane-bound $\alpha$-synuclein forms a complex with GCase, which lowers its activity. The $\alpha$-synuclein-GCase complex also negatively affects the degradation of $\alpha$-synuclein in the lysosomes. 
Table 2.3: Parkinson's disease populations screened for GBA mutations since 2014.

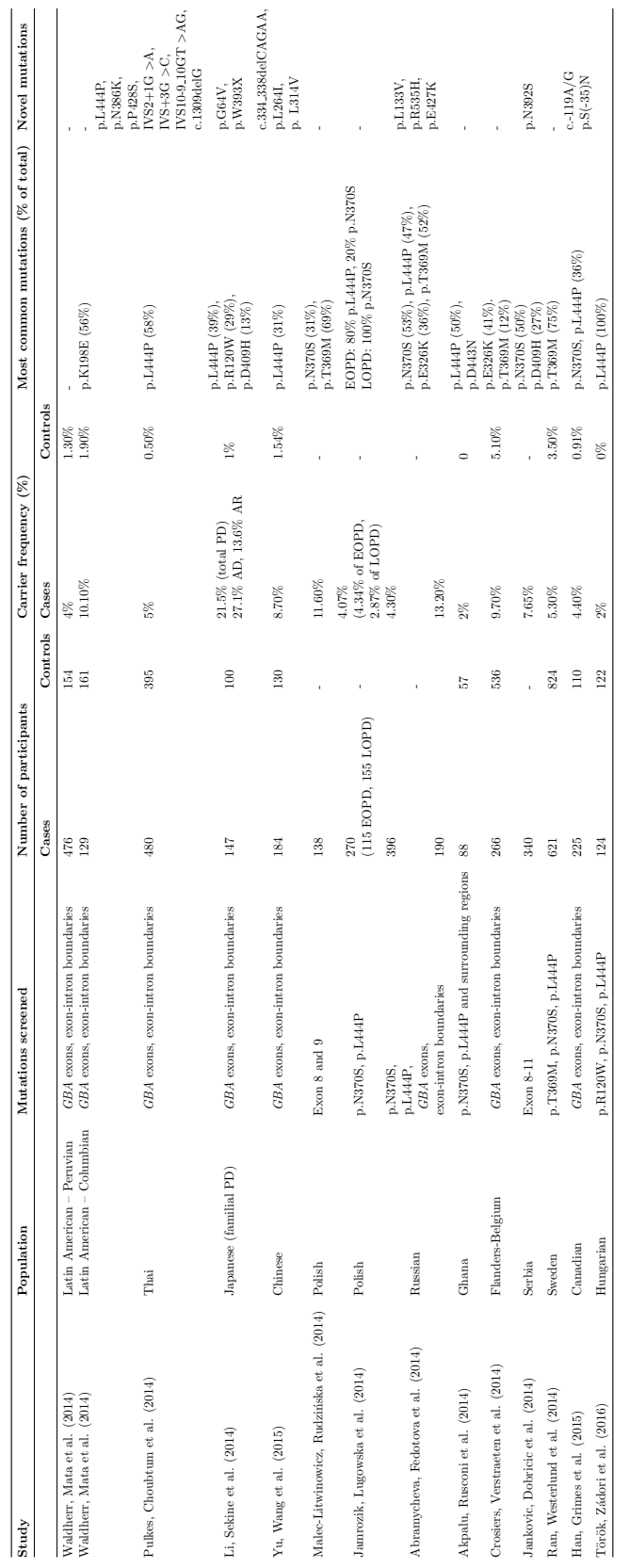


Therapeutic strategies aimed at improving GCase activity in the brain also have promising effects on $\alpha$-synuclein levels. Fish models show that in zebrafish (that has a genome without the $S N C A$ gene), the loss of $G B A$ function still causes significant neuronopathology and ubiquitin-inclusions in dopaminergic neurons. Conversely, rice fish show that an absence of GCase activity, accompanied by $\alpha$-synuclein accumulation in the neuron, is not lethal and that the life-span of the fish cannot be extended by preventing $\alpha$-synuclein pathology. The GCase- $\alpha$-synuclein cascade is thus more complex than a mere lack of substrate degradation and accumulation.

The recent boom in publications on GCase- $\alpha$-synuclein interactions has unravelled some of the other players involved in dopaminergic neuron death. In models of type $2 \mathrm{GD}$, significant Alzheimer's disease pathology ( $\beta$-amyloid and APP) co-occurs with the traditional $\alpha$-synuclein pathology. The neurological complications of type $2 \mathrm{GD}$, and perhaps $G B A-\mathrm{PD}$, may involve a multitude of accumulation-prone proteins. Mutant GCase impairs synaptic dopamine release through altering SNARE protein functions and reducing synaptic plasticity. LIMP-2 is moving to the forefront of the cascade. Normally, LIMP-2 is involved in the trafficking of GCase to the lysosomes. Both mutations in the SCARB2 gene, that encodes LIMP-2, and in GBA can impair the LIMP-2 dependent GCase trafficking. Restoring GCase function also augments pathology due to LIMP-2 dysfunction. SCARB2 mutations are also associated with myoclonic epilepsy - which occurs in the spectrum of neurological involvement in GD.

GBA-PD neurotoxicity may be exacerbated by increased neuro-inflammation and oxidative stress. A reduction in GCase function increases ROS and decreases cellular glutathione levels. ROS production and GCase misfolding are aggravated by deregulation of divalent metal ions $\left(\mathrm{Ca}^{2+}, \mathrm{Fe}^{2+}\right.$, and $\left.\mathrm{Zn}^{2+}\right)$. Glutathione supplementation with $N$-acetylcysteine and chelation of metal ions show some therapeutic benefit. The activation of Gaucher cells initiates the inflammatory cascade. Reactive astrocytosis, gliosis, and the release of various chemokines and cytokines worsen neuropathology. The accumulation of neurotoxic substrates is another factor. Parkin, a ubiquitin-ligase, is a well-known cause of familial PD. Mutant GCase interacts with Parkin in a competitive fashion, which reduces Parkin's ability to metabolize its endogenous substrates. Two tested substrates, PARIS and ARTS, are neurotoxic in high doses.

In addition to traditional mouse models, Gaucher cellular models are popular in recent studies. Models include fibroblasts and induced pluripotent stem cells used to manufacture dopaminergic neurons or macrophages. All 
the cellular models mimicked some of the pathology related to reduced GCase activity. Importantly, the iPSC-derived cells retained some of the phenotypical presentation of the donor. A study on iPSC-derived neurons from identical twins with $G B A$ mutations disconcordant for PD showed that although both twins had reduced GCase activity, the GCase activity of the affected twin was even lower and monoamine oxidase-B was up-regulated. These models represent a human alternative for animal models in which to conduct pre-clinical screening of new therapeutic compounds.

The therapy of GD is based on three strategies. Traditional enzyme replacement therapy forms the backbone of the GD treatment plan. However, the recombinant enzyme cannot cross the blood brain barrier, and thus has no influence on neurological symptoms. Reducing the production of glucocerebroside, through substrate-reduction therapy, forms the second strategy. Although miglustat, the hallmark SRT drug, is centrally available it does not reduce GD neuronopathology. The newest strategy involves correcting the folding and preventing degradation of mutant GCase with small molecule chaperones. This strategy increases reserve GCase activity. Ambroxol and isofagomine are promising drugs for GD and GBA-PD. These drugs also show a reduction in $\alpha$-synuclein accumulation in the absence of $G B A$ mutations - making it a promising strategy for the PD population as a whole. The effects of the chaperones are not limited to GCase improvement. Ambroxol also improves general lysosomal function through up-regulating a larger network of genes. Another therapeutic strategy involves preventing endogenous heat-shock proteins from recognizing GCase and so increase enzymatic activity. The effect of celastrol, the histone deacetylase inhibitors and direct inhibition of hsp-27 and ERdj3 works on this principle. As our understanding of $G B A$-associated neuropathology continues to expand, therapeutic options follow suit.

Our understanding of the clinical aspects of $G B A-\mathrm{PD}$ has also improved. Cognition is a major aspect of $G B A-P D$ management. Specific aspects of memory, particularly visual memory difficulties, and psychological disturbances are more common among GBA-PD individuals. The olfactory and visual function is also more severely affected in $G B A$ mutation carriers. $G B A-\mathrm{PD}$ patients are more prone to develop abnormal saccades and retinal thinning. $G B A$-PD patients can present with the full spectrum of PD motor symptoms. Both tremor-predominant and akinetic-rigid subtypes of $G B A$-PD have been observed. In general, $G B A$-mutations reduce the age-of-onset of $\mathrm{PD}$, with a gene-dosage effect. $G B A$-PD occurs in both sporadic- and familial PD cases. Frequently reported non-motor symptoms - constipation, orthostatic hypoten- 
sion and sexual dysfunction - do not appear to be more frequent in $G B A$ mutation carriers vs. the general PD population. However, fatigue is common in both GD and GBA-PD. Brain imaging studies showed asymmetrical radio ligand uptake in dopaminergic neurons from $G B A$-PD participants and white matter abnormalities in comparison to controls and other types of PD. Other types of brain scans could not differentiate between $G B A-\mathrm{PD}$ and idiopathic PD.

$G B A$ mutations appear to selectively increase the risk for synucleinopathies, like PD and BLD. Multiple System Atrophy is an exception to this rule. In MSA, the primary $\alpha$-synuclein pathology occurs in the oligodendrocytes - not in the neurons. $G B A$-susceptibility may be cell type specific. The link between $G B A$ and tauopathies, like Progressive Supranuclear Palsy and Corticobasal degeneration, is unconvincing. Reports of $G B A$ and $\mathrm{AD}$ are conflicting. However, given that Alzheimer's pathology was observed in GD models and that $\mathrm{AD}$ is not purely a tauopathy, this link is open for further investigation. The list of pathologic $G B A$ mutations - and global populations affected by $G B A-\mathrm{PD}$ continues to grow.

In conclusion, recent studies have highlighted the importance of GCase in $\alpha$-synuclein pathology and the broader neurotoxic cascade. As new players are identified - our understanding of this biological cascade, and possibly our therapies, continue to improve. This review provides a holistic overview of recent neuropathologic, clinical and genetic developments in the field.

\subsection{Acknowledgements:}

The authors wish to thank Prof Francois van der Westhuizen, Prof Huw Morris and Dr Silke Arndt for critically reading this manuscript and Vernon Joyce for the design of the graphical abstract. MB is funded by the National Research Foundation of South Africa (Grant specific reference number 89230). All views expressed in this review are those of the authors and not of the NRF. 



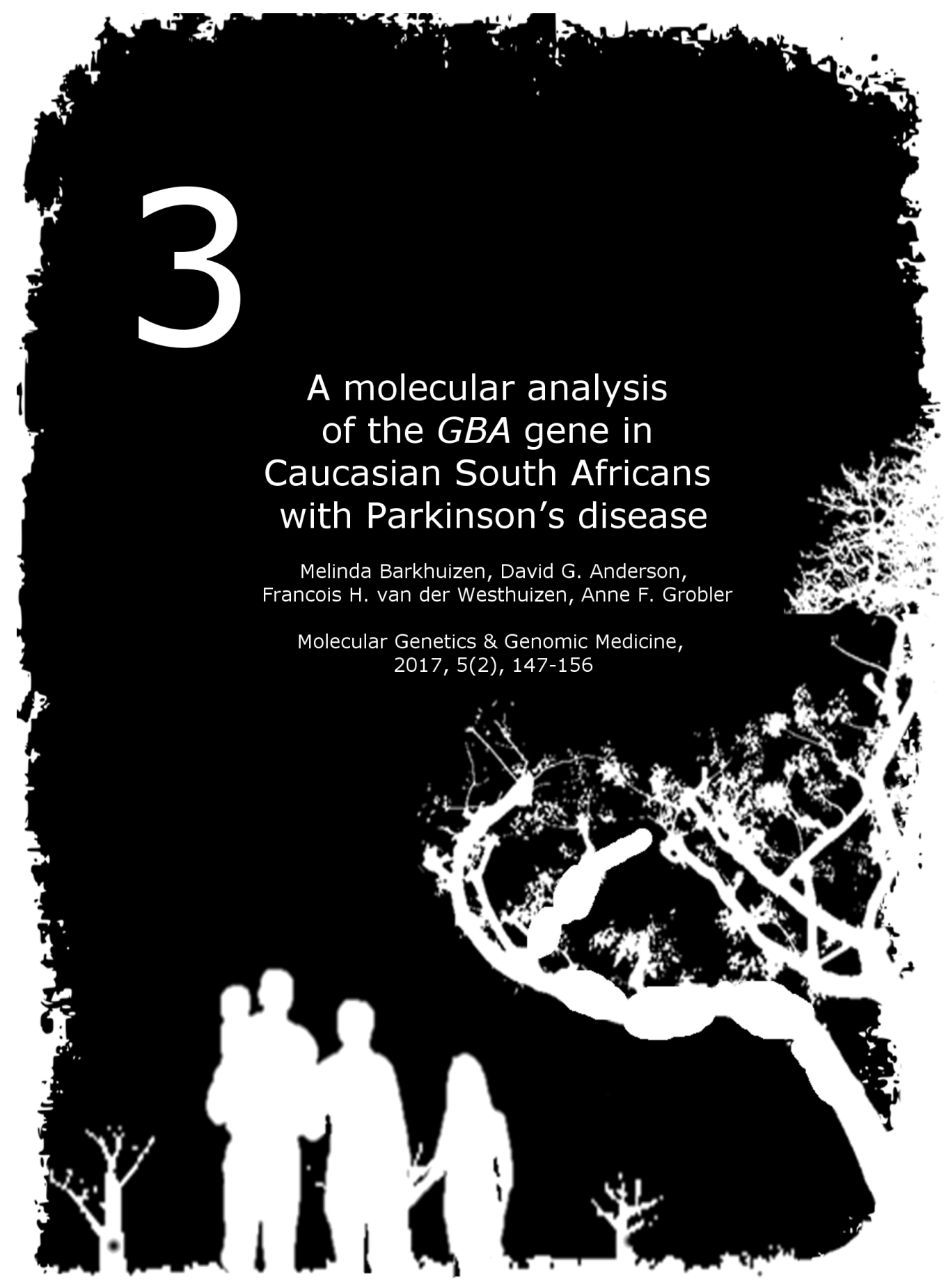




\title{
A molecular analysis of the $G B A$ gene in Caucasian South Africans with Parkinson's disease
}

Melinda Barkhuizen, David G. Anderson, Francois H. van der Westhuizen, Anne F. Grobler

Molecular Genetics 83 Genomic Medicine, 2017, 5, 147-156

\begin{abstract}
:
Background: The molecular basis of Parkinson's disease in South African population groups remains elusive. To date, substitutions in the GBA gene are the most common large-effect genetic risk factor for Parkinson's disease. The primary objective of this study was to determine the prevalence of $G B A$ substitutions in South Africans with idiopathic Parkinson's disease.
\end{abstract}

Methods: Participants were recruited from tertiary hospitals in the Gauteng Province in South Africa. All participants were screened for substitutions in $G B A$ exon 8-11 and the full coding region was analysed in 20 participants. Peripheral $\beta$-glucocerebrosidase enzymatic activity of $G B A$-carriers was measured in mixed leukocytes.

Results: 105 Caucasian Parkinson's disease participants (82.7\% Afrikaner) with an average age of disease onset of $61.9 \pm 12.2$ years and 40 controls (age $73.4 \pm 12.4$ years) were included. Heterozygous $G B A$ substitutions were identified in $12.38 \%$ of affected participants (p.G35A, p.E326K, p.I368T, p.T369M, p.N370S, p.P387L and p.K441N) and 5.00\% of controls (p.E326K and p.T369M). The substitutions ranged from predicted benign to moderately damaging, with p.E326K and p.T369M most prevalent, followed by the Afrikaner Gaucher disease substitution p.P387L. Severe Gaucher disease mutations, like p.L444P, were absent in this cohort. Enzyme activity analysis revealed a non-significant reduction in the GBA-Parkinson's disease individuals $(14.49 \pm 2.30 \mathrm{nmol} / \mathrm{hr} / \mathrm{mg}$ protein vs. $15.98 \pm 3.06 \mathrm{nmol} / \mathrm{hr} / \mathrm{mg}$ in control samples). GBA substitutions occur in both young-onset and late-onset Parkinson's cases in the cohort.

Conclusion: Mild GBA substitutions that may not cause Gaucher disease were a common risk factor for Parkinson's disease in the participant group. Keywords: Glucocerebrosidase, Afrikaner, Parkinson's disease, Gaucher disease, genetic screening, modelling 


\subsection{Introduction:}

Parkinson's disease (PD) is globally the $2^{\text {nd }}$ most common neurodegenerative disorder. It is characterized by a loss of dopaminergic neurons in the substantia nigra. The formation of abnormal intraneuronal aggregates, termed Lewy bodies, is the main neuropathological hallmark of PD. Lewy bodies predominantly consist of insoluble $\alpha$-synuclein (Dauer and Przedborski 2003). The aetiology of PD remains largely unknown. Classic Mendelian genes account for less than $10 \%$ of all cases (Lesage 2015). Multifactorial interactions between genetic risk factors and environmental exposures likely account for the majority of PD cases observed (Bekris, Mata et al. 2010). Cumulative genetic risk reduces the age-of-onset of PD. Meta-analyses of genome-wide association studies have identified 28 risk loci associated with PD (Nalls, Escott-Price et al. 2015). Of these, the most statistically significant signals associated are common substitutions located close to the SNCA (OMIM: 163890), LRRK2 (OMIM: 609007), MAPT (OMIM: 157140) genes and low frequency coding substitutions in the GBA (OMIM: 606463) gene (Benitez, Davis et al. 2016).

$G B A$ substitutions are the most common large-effect risk factor for $\mathrm{PD}$ globally. The $G B A$ gene encodes the lysosomal hydrolase $\beta$-glucocerebrosidase. Homozygous or compound heterozygous $G B A$ substitutions cause the lysosomal storage disorder Gaucher disease (GD), whilst both homozygous and heterozygous substitutions increase PD susceptibility (Sidransky, Nalls et al. 2009). GBA substitutions have been reported in 10.7-31.3\% of Ashkenazi Jewish PD cases and in between 2.3 and $9.4 \%$ of PD cases from other ethnic origins (Sidransky and Lopez 2012).

The GBA gene is located on chromosome 1q22. The gene consists of 11 exons and 10 introns, spanning 7.6-kb of sequence. A highly homologous pseudogene is located $16 \mathrm{~kb}$ downstream. Almost 300 unique $G B A$ substitutions have been identified in patients with GD, including missense, nonsense, and frame-shift substitutions as well as insertions, deletions, and complex alleles (Hruska, LaMarca et al. 2008). Approximately $70 \%$ of all the reported substitutions, including the two most common pathogenic substitutions, p.N370S and p.L444P, are located in exons 9-10 of the GBA gene (Asselta, Rimoldi et al. 2014).

The $\beta$-glucocerebrosidase protein has a direct interaction with $\alpha$-synuclein (Yap, Gruschus et al. 2011). Lower $\beta$-glucocerebrosidase activity has been reported in the brain-regions affected by $\alpha$-synuclein pathology - even in sporadic PD cases without $G B A$-substitutions (Gegg, Burke et al. 2012, Chiasserini, 
Paciotti et al. 2015). There is a feed-forward loop between $\beta$-glucocerebrosidase and $\alpha$-synuclein, where a reduction in enzymatic activity increases $\alpha$-synuclein accumulation and fibrillization. This further reduces the activity of the $\beta$ glucocerebrosidase enzyme (Mazzulli, Xu et al. 2011). It is estimated that carriers of heterozygous $G B A$ substitutions have a $1.5 \%$ probability of developing PD by 60 years of age and a $7.7 \%$ chance of developing PD by age 80 (Alcalay, Dinur et al. 2014). Clinically, $G B A$-associated PD cases resemble idiopathic PD. However, GBA-PD patients are more prone to cognitive decline and they may have a younger average age-of-onset of PD (Alcalay, Dinur et al. 2014, Brockmann, Srulijes et al. 2015). A loss of smell is also frequently reported by $G B A$-substitution carriers, even in individuals without PD (Beavan, McNeill et al. 2015).

The 2014 mid-year population estimation reports indicate that South Africa has 55 million residents, of which $8.3 \%$ are Caucasian. Additionally, $8 \%$ of the total South African population is estimated to be older than 60 years (StatsSA 2015). The Caucasian South African population predominantly consists of Afrikaans-speaking individuals (63\%) and English-speaking individuals (StatsSA 2012). The Afrikaans-speaking Caucasian population - hereafter called Afrikaners - is a unique ethnic group in South Africa with well-documented ancestral records spanning a period of over 350 years. They are mainly descended from Dutch, German and French settlers to RSA in the 17th and 18th centuries. There are also minor genetic contributions by British descendants and non-European slaves (Greeff 2007). They have a relatively small gene pool, and several founder effects exist within the population. Previous genealogical research has shown that 40/48 Afrikaner families with PD can be traced back to a single founder couple (Geldenhuys, Glanzmann et al. 2014). Prior genetic studies in South Africa have concluded that the known Mendelian PD genes are rare in South African population groups (Bardien, Keyser et al. 2009, Keyser, Van Der Merwe et al. 2009, Bardien, Marsberg et al. 2010, Keyser, Lesage et al. 2010, Keyser, Lombard et al. 2010, Keyser, Oppon et al. 2011, Haylett, Keyser et al. 2012, Blanckenberg, Ntsapi et al. 2014). However, the frequencies of genetic susceptibility substitutions in the local population groups have not yet been established. In this study, we explored the frequency of $G B A$-substitutions in a Caucasian South African cohort diagnosed with PD. The possible pathogenicity of the substitutions was assessed with computational prediction algorithms, $\beta$-glucocerebrosidase enzymatic activity analysis and through modelling the positions of these substitutions relative to the interaction interface between the $\beta$-glucocerebrosidase and $\alpha$-synuclein proteins. 


\subsection{Materials and methods:}

\subsubsection{Ethical compliance:}

This project was conducted under ethics approval nr. NWU-00051-14-A1 of the Health Research Ethics Committee of the North-West University in South Africa.

\subsubsection{Participant selection:}

Hundred-and-five Caucasian South African PD patients were recruited through referring neurologists at tertiary hospitals in the Gauteng province of South Africa. All participants fulfilled the UK PD Society Brain Bank Research criteria for diagnosis of PD (Gibb and Lees 1988) as assessed by a registered neurologist. The participants were all at least second generation South African.

Forty non-related aged Caucasian control participants were recruited through medical practitioners from the Gauteng province in South Africa. Controls were excluded if they had a positive family history of PD or GD, or if they reported having more than one of the cardinal signs of PD. All participants provided written informed consent.

\subsubsection{Sequencing and data analysis:}

PCR amplification of the exon 8-11 fragment of GBA, followed by Sanger sequencing of the exons and exon-flanking regions was done in all PD-participants and controls with previously described primers (Sato, Morgan et al. 2005). In addition, in twenty PD-participants, without mutations in exon 8-11, the remaining $G B A$ exons were screened in fragments from exon 1-5 and exon 5-7 with primers previously described by Stone, Tayebi et al. (2000). Direct sequencing was performed using the BigDye Terminator Sequence Ready Reaction kit version 3.1 (Applied Biosystems) and analyzed on a $3130 \times 1$ Genetic Analyzer (Applied Biosystems) at Inqaba Biotechnical Industries (Pretoria, South Africa). CLC Main Workbench version 7.6.2 software was used for the analysis of the sequencing electropherograms. The amino acid positions are described using the classic $G B A$ nomenclature based on the amino acid position in the mature enzyme. This system omits the first 39 amino acid residues of reference sequence NP_000148.2 (Hruska, LaMarca et al. 2008). Samples with 
nonsynonymous substitutions were resequenced for confirmation.

The pathogenicity of nonsynonymous substitutions was predicted with seven different online algorithms - six function prediction scores (MutPred, MutationTaster-2, Polyphen-2, PhD-SNP, SIFT, SNP\&GO) and an ensemble score (CADD). Polyphen-2 evaluates eight protein sequence features, three protein structure features (scores $>0.5$ are deleterious), SIFT assesses protein sequence conservation among homologs (scores $>0.95$ are deleterious), MutPred combines the SIFT score with an evaluation of 14 structural and functional properties (scores $>0.5$ are deleterious); MutationTaster-2 evaluates DNA sequence conservation, splice site prediction, mRNA stability prediction and protein feature annotations (scores $>0.5$ are deleterious); PhD-SNP and SNP\&GO use a support vector machine classification based on the protein sequence and profile and the CADD-algorithm uses distinct substitution annotation retrieved from Ensembl Variant Effect Predictor, data from the ENCODE project and information from UCSC genome browser tracks (scores $>15$ are deleterious) (Frousios, Iliopoulos et al. 2013, Dong, Wei et al. 2015).

\subsubsection{Enzyme activity analysis:}

The $\beta$-glucocerebrosidase activity of extracted mixed leukocytes was measured with a previously described assay that uses 4-methylumbelliferyl $\beta$ glucopyranoside (4-MUG) as a substrate in the presence of sodium taurocholate $(1.88 \mathrm{~g} / \mathrm{l})$ (Nakagawa, Kumin et al. 1982). The enzymatic assays were performed by Mr Iain Sinclair at the National Health Laboratory Service (Braamfontein, South Africa) as part of a Gaucher disease testing protocol.

\subsubsection{Molecular modeling:}

$\mathrm{p} K_{a}$ values for $\beta$-glucocerebrosidase were set at 5.5 and 7.4 with PROPKA 2.0. A peptide consisting of residues $115-140$ of $\alpha$-synuclein was docked interactively and minimized using Maestro/PIPER (Schrödinger Inc.) (Kozakov, Brenke et al. 2006) to the $\beta$-glucocerebrosidase x-ray structure (PDB ID: 3GXM, chain C), similar to predictions previously conducted by Yap, Gruschus et al. (2011). Interactions were analyzed with the protein-protein interaction analysis tools and the interaction fingerprint analysis of Maestro (Schrödinger Inc.). Figures were made with PyMOL (Schrödinger Inc.). 


\subsubsection{Statistical analysis:}

For differences of the prevalence of $G B A$ mutations between PD and control participants, a Chi-square test was used. Enzymatic activity values were assessed for normality with a Shapiro-Wilk test and statistical significance was calculated with a t-test. Statistical significance was set at p-values smaller than 0.05 .

\subsection{Results:}

\subsubsection{Participant characteristics:}

The affected PD participants had an average age-of-onset $61.9 \pm 12.2$ years with average disease duration of $7.5 \pm 6.8$ years prior to participating in this study. The gender ratio was 1:1.8 (female: male) and $82.7 \%$ of the participants were of self-reported Afrikaner descent. The remainder was English-speaking Caucasian South-Africans. None of the participants reported Ashkenazi Jewish Ancestry.

The control participants had an average age of $73.4 \pm 12.4$ years. We preferentially selected a neurologically normal older control population to reduce the amount of participants that may later develop PD. The controls were predominantly of self-reported Afrikaner descent (65\%) and the gender ratio of the controls was 1:1.8 (male: female).

\subsection{2 $G B A$ substitution prevalence:}

Nonsynonymous heterozygous $G B A$ substitutions (including p.E326K and p.T369M) were identified in $12.4 \%$ of the PD participants and $5.0 \%$ of controls ( $p>0.05)$. Putative GD-causing substitutions were identified in $5.7 \%$ of PD participants and $0 \%$ of controls $(\mathrm{p}>0.05)$, similar to frequencies previously reported in non-Ashkenazi Jewish PD patients (Sidransky, Nalls et al. 2009). The remainder of substitutions consisted of the non-GD causing polymorphisms: p.E326K and p.T369M. In the exon 8-11 fragment we identified: p.E326K (4.8\% of PD-participants, $2.5 \%$ of controls), p.I368T (1.0\% of PD-participants), p.T369M (1.9\% of PD-participants, $2.5 \%$ of controls), p.N370S (1.0\% of PDparticipants), p.P387L (1.9\% of PD-participants) and p.K441N (1.0\% of $\mathrm{PD}$-participants). Analysis of the remainder of the exon-flanking regions in a sub-set of participants identified one additional substitution, p.G35A (5.0\% of the subset of PD-participants). The characteristics of the $G B A$-substitution carriers are listed in table 3.1. Synonymous and intronic substitutions identified 
are listed in supplementary table 3.1 .

\subsubsection{Molecular Modelling:}

To shed more light on the possible pathological impact of the substitutions identified in the study, the influence that these amino acid substitutions could have on the binding interface between $\beta$-glucocerebrosidase and $\alpha$-synuclein was investigated with molecular modelling.

The interactions between the wild-type $\beta$-glucocerebrosidase and wild-type $\alpha$-synuclein proteins were determined first to determine whether any altered amino acids are directly involved in the protein-protein interactions (figure 3.1).

Next, wild-type $\alpha$-synuclein was docked into a series of $\beta$-glucocerebrosidase proteins with a single amino acid substitution. The substituted amino acids represent each variation identified in the participants. The modelling was performed to determine whether these substitutions alter the binding formation of the two proteins. Docking simulations were executed at pH 5.5 (lysosomal $\mathrm{pH}$ ) and $\mathrm{pH} 7$ (endoplasmic reticulum $\mathrm{pH}$ ). These $\mathrm{pHs}$ were chosen since $\beta$-glucocerebrosidase is secreted in the endoplasmic reticulum, but it has to be transported to the lysosomal compartment to exert its enzymatic activity. The p.N370S GD mutation causes early protein misfolding in the endoplasmic reticulum. This prevents the trafficking of $\beta$-glucocerebrosidase to the lysosome, but does not reduce the intrinsic catalytic activity of the enzyme (Maegawa, Tropak et al. 2009). The effect of the other substitutions identified in this study is less clear. Substitution of amino acids in $\beta$-glucocerebrosidase only caused minor alterations were in the predicted binding conformation of $\alpha$-synuclein and none of the glucocerebrosidase substitutions were located directly on the interaction interface (supplementary figure 3.1).

The analysis of the molecular interaction fingerprints between the top 30 docked $\alpha$-synuclein poses in the substitution $\beta$-glucocerebrosidase proteins at both $\mathrm{pH} 5.5$ and $\mathrm{pH} 7$ showed that the residues that were predicted to contribute the majority of the binding energy between the two proteins remained fairly constant among all the glucocerebrosidase substitutions modelled. Thus the amino acid substitution did not disrupt an important interaction between the two proteins.

In the majority of the molecular finger print analyses, $\beta$-glucocerebrosidase residues Asp-127, Lys-194, Ser-237, Asn-238, Ser-242, Glu-340, Ser-345, Gln- 
Table 3.1: Non-synonymous substitutions identified

a. Traditional protein numbering, which emits the first 39 amino acid residues

b. Protein numbering according to reference sequence NP_000148.2

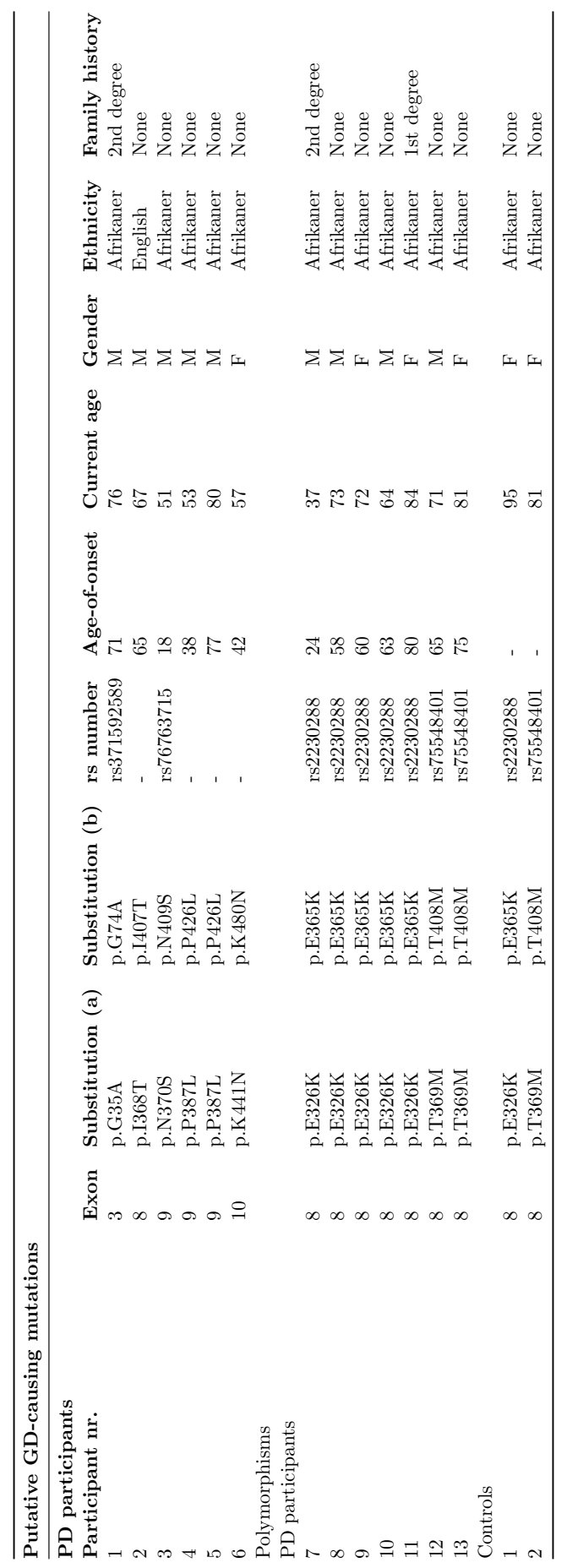


350, Ser-351 form polar interactions with the majority of $\alpha$-synuclein poses, regardless of pH. Residues Lys-346, Glu-349, Arg-353, Asp-358 and Arg-395 form both polar and charged interactions with $\alpha$-synuclein at neutral and lysosomal pH. At pH 5.5 additional polar and charged interactions are contributed by His-290 and Glu-235 respectively. Residues Leu-240, Leu-241, Pro-245, Pro253, Leu-286, Leu-287, Pro-289, Leu-314, Leu-317, Ala-318, Pro-319, Val-394 and Val-398 form hydrophobic interactions, whilst residues Tyr-244, Phe-246, Trp-291, Tyr-313, Phe-316, Phe-347, Trp-348 and Phe-397 form both hydrophobic and aromatic interactions with $\alpha$-synuclein.

Lastly, the protein-protein interactions between the best wild-type $\alpha$ synuclein $(\alpha \mathrm{S})$ pose and wild-type $\beta$-glucocerebrosidase $(\mathrm{GC})$ were measured to identify individual residues of $\alpha$-synuclein that interacts with $\beta$-glucocerebrosidase. At $\mathrm{pH} 5.5, \alpha \mathrm{S}$ Val-118 interacts with GC residues 240-241. $\alpha \mathrm{S}$ Glu-123 interacts with GC Ser-237 and Leu-241, $\alpha$ Syn residues 124-125 interact with GC Leu-286, $\alpha \mathrm{S}$ Tyr-125 also interacts with GC Leu-317 and Ala-318. $\alpha \mathrm{S}$ residues 128 - 132 interacts with GCase residues 241, 244-246, 313-314. $\alpha \mathrm{S}$ Glu-131 also interacts with GC residues 127-128 and 392. $\alpha \mathrm{S}$ residues 131134 interact with GC 395-397. $\alpha$ S residues 131-140 interacts with GC 345-353. The substitution amino acids in this study (Gly-35, Glu-326, Ile-368, Thr-369, Asn-370, Pro-387 and Lys-441) do not appear to have an important role in the GCase- $\alpha$-synuclein interaction.

\subsubsection{Impact of substitutions on $G B A$ structure and function:}

The putative structural impact of the amino-acid substitution was analyzed in silico by visualization of the $3 \mathrm{D}$ conformation of the $\beta$-glucocerebrosidase protein structure after energy minimization with Maestro/PIPER (Schrödinger Inc.) with $\alpha$-synuclein docked. The results of the minimization at $\mathrm{pH} 5.5$ and $\mathrm{pH} 7$ are shown in figure 3.2. The substitutions were not predicted to cause large changes in the overall $\beta$-glucocerebrosidase protein folding, but it resulted in subtle differences in side-chain orientation (supplementary figure 3.2). The molecular modelling did not identify a clear mechanism of pathogenicity of the amino-acid substitutions.

Thus we explored the predicted structural and functional consequences further with seven web-based computational pathogenicity prediction tools (table 3.2). The p.P387L and p.K441N substitutions were predicted to be benign by $7 / 7$ tools, p.E326K and p.T369M were predicted to be benign by $4 / 7$ tools. P.G35A and p.I368T were predicted to be damaging or possibly damaging 


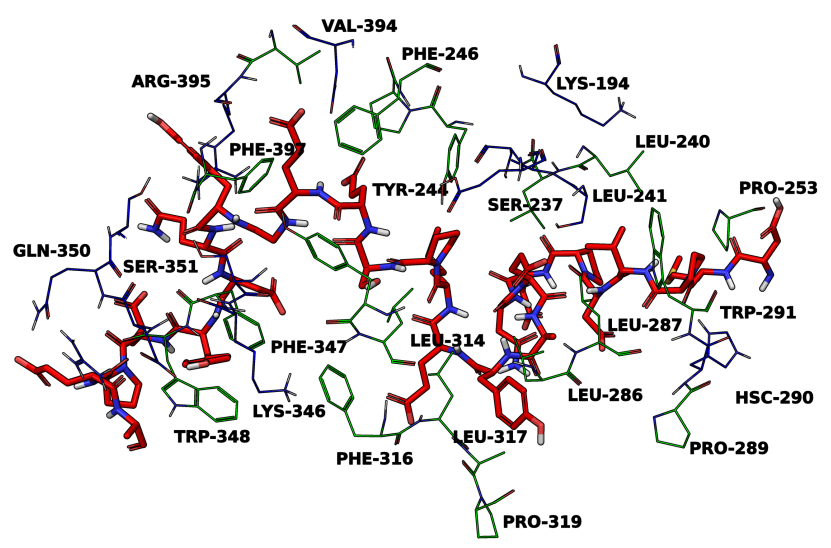

Figure 3.1: Important interactions between glucocerebrosidase and $\boldsymbol{\alpha}$-synuclein at $\mathrm{pH}$ 5.5.Image guide: $\boldsymbol{\alpha}$-synuclein residues 114-140 (red) and selected polar and charged residues (blue), hydrophobic and aromatic residues (green) of $\boldsymbol{\beta}$-glucocerebrosidase 3GXM chain C.

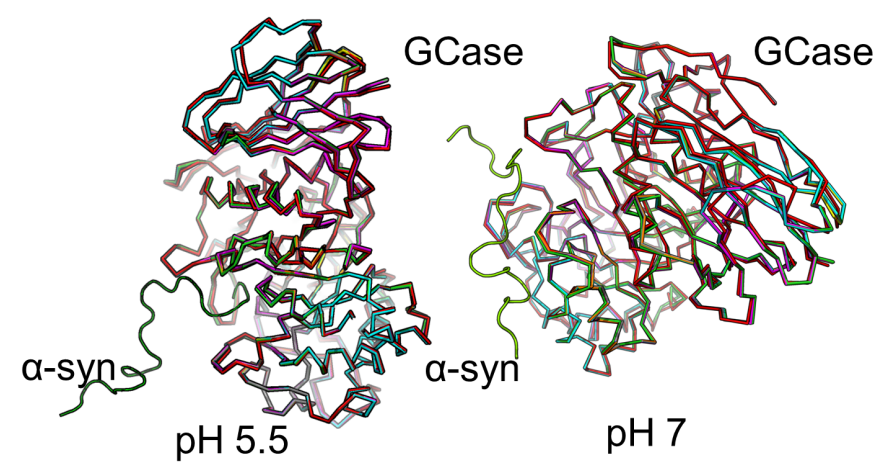

Figure 3.2: Overall conformation of the substituted $\beta$-glucocerebrosidase receptor after minimization for docking with $\alpha$-synuclein at $\mathrm{pH} 5.5$ and $\mathrm{pH}$ 7. Image guide: wild type $=$ red, p.G35A $=$ yellow, p.E326K $=$ orange, $\mathrm{p} \cdot \mathrm{I} 368 \mathrm{~T}=$ grey, $\mathrm{p} . \mathrm{T} 369 \mathrm{M}=$ purple, p.N370S $=$ lime, p.P387L = cyan, $\mathrm{K} 441 \mathrm{~N}=$ magenta. $\alpha$-synuclein is displayed as a green ribbon. 
Table 3.2: Leukocyte glucocerebrosidase activity and pathogenicity predictions of the substitutions identified. a. Traditional protein numbering, which emits the first 39 amino acid residues

b. Protein numbering according to reference sequence NP_000148.2

Damaging or possibly damaging scores are indicated in bold.

\begin{tabular}{|c|c|c|c|c|c|c|c|c|c|}
\hline Predicted proteina(b) & $\begin{array}{l}\text { GBA-carrier } \\
\text { enzymatic activity } \\
\text { in mond/lin/mg protein } \\
\text { (PD-participant nr) }\end{array}$ & $\begin{array}{l}\text { Intra-assay control } \\
\text { ennymatic cativity } \\
\text { in mmol/hr/mg protein }\end{array}$ & $\begin{array}{l}\text { ScaleddADD v1.3 score } \\
(>15=\text { deleterious })\end{array}$ & $\begin{array}{l}\text { MutPred score } \\
(>0.5=\text { deleterious })\end{array}$ & MutationTaster-2 prediction & PhD-SNP score & $\begin{array}{l}\begin{array}{l}\text { PolyPhen-2 prediction } \\
\text { (score) }\end{array} \\
\text { (a) }\end{array}$ & $\begin{array}{l}\text { SIFT Prediction } \\
\text { (score) }\end{array}$ & SNP\&:GO score \\
\hline \multirow{7}{*}{ 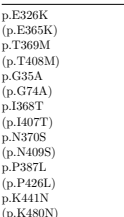 } & $17.24(10)$ & 16.6 & 17.33 & 0.521 & Disease causing & Neutral & $\begin{array}{l}\operatorname{Benign}(0.15) \\
\text {. }\end{array}$ & Tolerated $(0.43)$ & Neutral \\
\hline & \multirow{3}{*}{$\begin{array}{l}17.55(12) \\
13.63(13) \\
11.16(1)\end{array}$} & $\begin{array}{l}18.89 \\
1751\end{array}$ & 22.2 & 0.502 & Polymorphism & Neutral & Benign (0.275) & Tolerated $(0.12)$ & Disease \\
\hline & & $\begin{array}{l}10.16 \\
10.16 \\
10\end{array}$ & 23.5 & 0.809 & Disease causing & Neutral & Possibly damaging $(0.91)$ & Tolerated $(0.06)$ & Disease \\
\hline & & & 26.6 & 0.847 & Disease causing & Neutral & Probably damaging $(0.977)$ & Tolerated $(0.07)$ & Disease \\
\hline & $12.29(3)$ & 11.65 & 22.7 & 0.876 & Disease causing & Disease & Possibly damaging (0.607) & Damaging (0.02) & Disease \\
\hline & \multirow{2}{*}{$\begin{array}{l}16.96(4) \\
14.17(5) \\
12.55(6)\end{array}$} & $\begin{array}{l}17.51 \\
1808\end{array}$ & 8.4 & 0.44 & Polymorphism & Neutral & Benign (0) & Tolerated (0.31) & Neutral \\
\hline & & $\begin{array}{l}16.69 \\
16.6\end{array}$ & 10.04 & 0.441 & Polymorphism & Neutral & Benign (0) & Tolerated $(0.33)$ & Neutral \\
\hline
\end{tabular}

by $5 / 7$ tools and the common GD-causing mutation, p.N370S, was predicted to be damaging by $7 / 7$ algorithms. These results indicate that there were a mixture of probable-benign substitutions and damaging substitutions identified in the participant group. The MutPred tool indicated the top five molecular features which are predicted to be influenced by amino-acid substitution ( supplementary table 3.2) (Li, Krishnan et al. 2009). Among the actionable hypotheses, it was predicted that the p.E326K and p.I368T mutations could lead to a gain of a catalytic residue, p.T369M could lead to a loss of a catalytic residue and p.N370S and p.E326K could increase the glycosylation of the protein.

The in vitro effect of the substitutions on the catalytic activity of $\beta$ glucocerebrosidase was measured in mixed leucocytes from a peripheral blood sample (table 3.2). This measurement could not distinguish between enzymatic activity in participants and intra-assay control samples in our study.

\subsection{Discussion:}

Our reported mutation frequencies are similar to reported frequencies persons with PD from non-Ashkenazi Jewish descent (Sidransky, Nalls et al. 2009). The $G B A$ substitution spectrum in the South African population mainly consisted of mild damaging and possibly benign substitutions. We have only identified a single p.N370S carrier (rs76763715) and no p.L444P carriers. Globally, these 2 substitutions are considered to be the two most common $G B A$ substitutions in both GD and PD. As a result, numerous studies have only screened for those 2 substitutions (Sidransky, Nalls et al. 2009). 
In our cohort, the most common nonsynonymous substitutions were the polymorphisms p.E326K (rs2230288) and p.T369M (rs75548401). These substitutions are known to cause a mild reduction in enzymatic activity and not cause GD, even in the homozygous state. However, p.E326K has been reported to predispose to $\mathrm{PD}$ and it is also a common PD-associated substitution in other populations (Nichols, Pankratz et al. 2009, Horowitz, Pasmanik-Chor et al. 2011, Duran, Mencacci et al. 2013). Similarly, the p.T369M substitution has previously been identified in PD cases (Clark, Ross et al. 2007, Nichols, Pankratz et al. 2009, Benitez, Davis et al. 2016). Whilst it is known that severe GD-associated $G B A$ substitutions increase the risk of cognitive decline (Alcalay, Caccappolo et al. 2012, Winder-Rhodes, Evans et al. 2013), recent evidence indicated that the p.E326K substitution also increases the risk of working memory/executive function decline and visio-spatial memory impairment in PD (Mata, Leverenz et al. 2015).

The p.P387L substitution has previously been identified in an Afrikaner GD individual (Morar and Lane 1996). It was subsequently included in the GD genetic screening panel for Afrikaner individuals, but our computational predictions indicated that it may be a benign polymorphism. The p.G35A substitution (rs371592589) has been identified previously in a South African mixed ancestry individual with unknown PD or GD status. Another nonsynonymous substitution of the same amino acid, p.G35S, has been linked to GD (Rozenberg, Fox et al. 2006). The p.G35A carrier also had a $2^{\text {nd }}$ degree relative with PD. This substitution was predicted to be damaging by the majority of algorithms.

We also report two novel $G B A$ substitutions, p.I368T and p.K441N. The p.I368T substitution occurred in a late-onset sporadic PD case of English South African ancestry. It was predicted to be damaging by the majority of algorithms. The p.K441N substitution occurred in a sporadic young-onset Afrikaner participant. The substitution was predicted to be benign. Interestingly, modelling of the $\alpha$-synuclein - glucocerebrosidase interaction by Yap, Gruschus et al. (2011) has predicted that there is an electrostatic interaction between $\beta$-glucocerebrosidase Lys-441 and the negatively charged residues of $\alpha$-synuclein. Thus the p.K441N substitution could potentially cause PD by disrupting an interaction with $\alpha$-synuclein, rather than by reducing intrinsic enzymatic activity. We aimed to replicate their interaction between $\beta$-glucocerebrosidase and $\alpha$-synuclein, but Lys-441 did not contribute significantly to the protein-protein interaction in our simulations. 
Since we only screened for exon 8-11 in the majority of the cohort, it is possible that up to $30 \%$ of substitutions were not identified and that the true nonsynonymous substitution frequency could reach up to $16 \%$ of persons with $\mathrm{PD}$. In the overall frequency data we only report the nonsynonymous substitution frequency. Previous computational analysis shows that the majority of the intronic and synonymous substitutions - as well as a few exonic substitutions, may alter splicing (Barkhuizen, Anderson et al. 2016). However, this has not been verified in vitro and it would be worthwhile to pursue in the future with the rare intronic substitutions.

The peripheral $\beta$-glucocerebrosidase enzymatic activity of PD patients has been reported to be lower than in controls and $G B A$-heterozygotes had a lower enzymatic activity than non-carriers. These measurements were made with mass-spectrometry of dried blood spots (Alcalay, Levy et al. 2015, Glickman, Bodamer et al. 2015). In our study, the difference in enzyme activity was only slightly lower than the control values and it did not reach statistical significance $(\mathrm{p}>0.05)$. This could reflect that the substitution spectrum predominantly consists of mild substitutions or due to a small sample size. However, even the p.N370S heterozygote did not have a marked reduction in $\beta$-glucocerebrosidase activity. It is likely that the 4 -methylumbelliferyl $\beta$-glucopyranoside assay in leukocytes was not sensitive enough to identify individual $G B A$-carriers for routine clinical use. This correlates with observations by Kim, Jeon et al. (2016) who found that the leukocyte 4-methylumbelliferyl $\beta$-glucopyranoside assay cannot distinguish sporadic PD patients from controls in a cohort that was not genotyped for $G B A$ substitutions. In a genotyped cohort, Ortega, Torres et al. (2016) observed similar enzymatic activity in GBA-PD participants (16.0 $\mathrm{nmol} / \mathrm{hr} / \mathrm{mg}$ protein) than in our study $(14.49 \pm 2.30 \mathrm{nmol} / \mathrm{hr} / \mathrm{mg}$ protein). However, they observed much higher values in idiopathic PD $(28.5 \mathrm{nmol} / \mathrm{hr} / \mathrm{mg}$ protein) and asymptomatic $G B A$ carriers $(25.5 \mathrm{nmol} / \mathrm{hr} / \mathrm{mg}$ protein $)$, than in the intra-assay control subjects in our study $(15.98 \pm 3.06 \mathrm{nmol} / \mathrm{hr} / \mathrm{mg})$ and the controls a previous South African GD study $(13.71 \pm 2.85 \mathrm{nmol} / \mathrm{hr} / \mathrm{mg})$ (Arndt, Heitner et al. 2009) with similar methodology. Whether leucocyte $\beta$ glucocerebrosidase activity can reliably identify $G B A-\mathrm{PD}$ participants remains open for further testing, but in this study it did not distinguish $G B A-\mathrm{PD}$ from control individuals.

The age-of-onset of $\mathrm{PD}$ in the cohort was divided, with $G B A$ substitutions observed in both young-and late-onset cases. It is possible that confounding genetic factors drive the earlier age-of-onset in the young-onset cases, particularly as the majority of the substitutions are predicted to be benign and these 
participants have not been tested for other PD-related genes. Alternately, it is possible that the late-onset participants carry a protective modifier substitution.

The contribution of $G B A$ to PD in other South African population groups remains to be studied in the future. Genotyping of Black South Africans with Gaucher disease revealed substantial differences in the substitution spectrum in comparison to the Caucasian population groups (Arndt, Heitner et al. 2009). The study did not manage to enrol sufficient amounts of Black African, Mixed Ancestry or Indian ethnicity PD patients to make definite conclusions about other South African population groups.

\subsection{Conclusion:}

Mild $G B A$ substitutions may be a substantial risk factor for PD in the South African Caucasian populations. The prevalence of $G B A$ substitutions in the South African Afrikaner community is higher than previously thought. Since $G B A$ substitutions also increase the risk of cognitive decline, it may be clinically relevant to screen Afrikaner PD patients for $G B A$ substitutions.

\subsection{Acknowledgements:}

This work was funded by the DST/NWU Preclinical Drug Development Platform and MB was funded by the National Research Foundation of South Africa (Grant specific reference numbers 89230 and 98217). All views expressed in this review are those of the authors and not of the NRF. The authors wish to thank all the neurologists, nurses, administrative staff and M. Hall for assistance with the recruitment; AMPATH laboratories for generously drawing blood for this study; the team at Inqaba biotec, especially Dr C. Labuschagne and Dr S. Arndt, for advice and technical assistance; and Prof F. Steyn from the Statistical Consultation Services of the North-West University for the study design. 


\subsection{Supplementary material:}

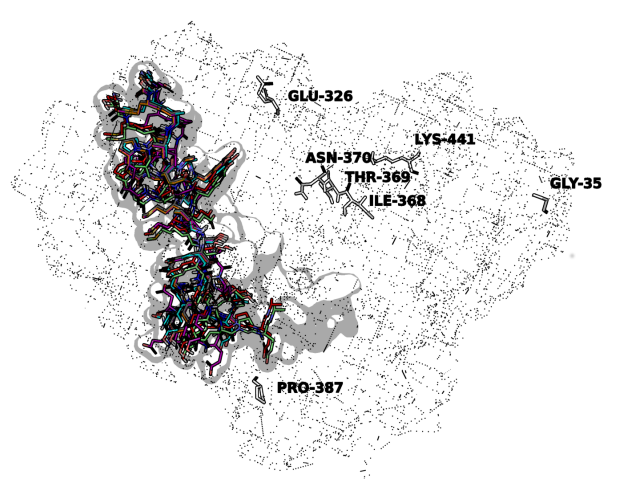

Supplementary figure 3.1: The top-poses of the $\alpha$-synuclein side-chain docked into substituted $\beta$-glucocerebrosidase, shown in relation to the position of substitutions identified. Image guide: The conformations of wild-type $\alpha$-synuclein docked into substituted $\beta$-glucocerebrosidase (wild-type $=$ red, p.G35A = yellow, p.E326K = orange, p.I368T = grey, p.T369M = purple, p.N370S = lime, p.P387L = cyan, K441N $=$ magenta. Selected $\beta$-glucocerebrosidase residues are shown in white). 


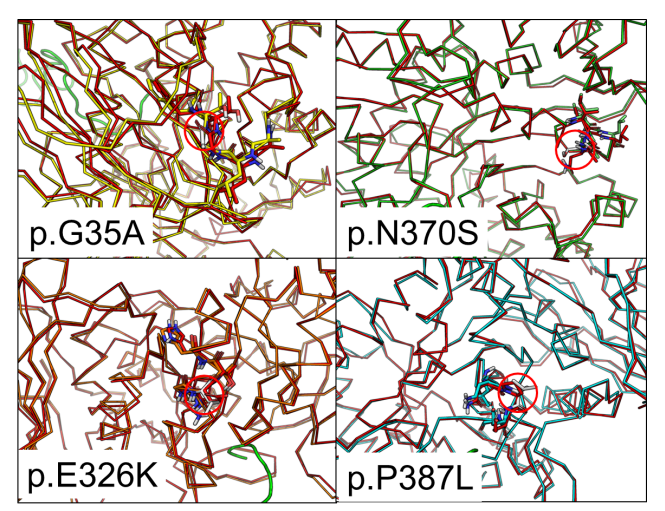

Supplementary figure 3.2: A magnified view of the conformation of selected substituted $\beta$-glucocerebrosidase receptors at $\mathrm{pH} 5.5$ docked with $\alpha$-synuclein. Image guide: wildtype $=$ red, p.G35A = yellow, p.E326K = orange, p.N370S = lime, p.P387L = cyan. The substituted amino acid is displayed in white and circled. $\alpha$-synuclein is displayed as a green ribbon.

Supplementary table 3.1: Synonymous and intronic variants identified

a. cDNA numbering relative to reference sequence NM000157.3 and starting at first nucleotide of translation initiation codon.

\begin{tabular}{llll}
\hline Variant & rs number & \% of patients & \% of controls \\
\hline c.1225-34C $>$ A & rs 3115534 & $93.3 \%$ & $100 \%$ \\
c.1388+141A $>$ G & rs28373017 & $32.3 \%$ & $42.5 \%$ \\
c. $1388+94 \mathrm{G}>\mathrm{A}$ & $\mathrm{rs} 12752133$ & $6.7 \%$ & $2.5 \%$ \\
c. $1506-10 \mathrm{~T}>\mathrm{G}$ & $\mathrm{rs} 577529715$ & $1.0 \%$ & $0.0 \%$ \\
c. $1389-68 \mathrm{~T}>\mathrm{C}$ & $\mathrm{rs} 143255568$ & $2.9 \%$ & $0.0 \%$ \\
p.L249= & - & $1.0 \%$ & $0.0 \%$ \\
\hline
\end{tabular}


Supplementary table 3.2: MutPred predictions of functional consequences of the mutations (Li et al., 2009).

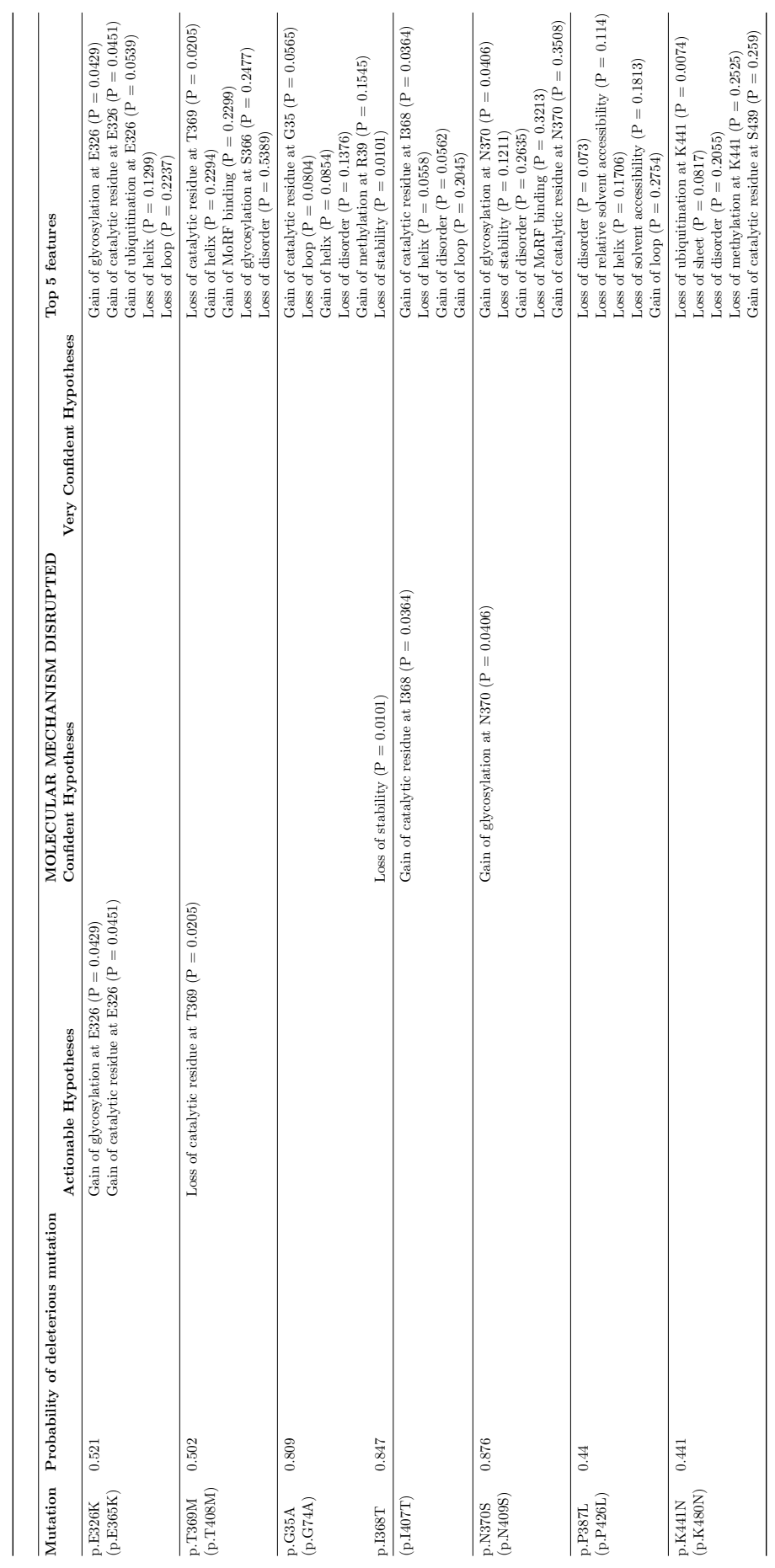






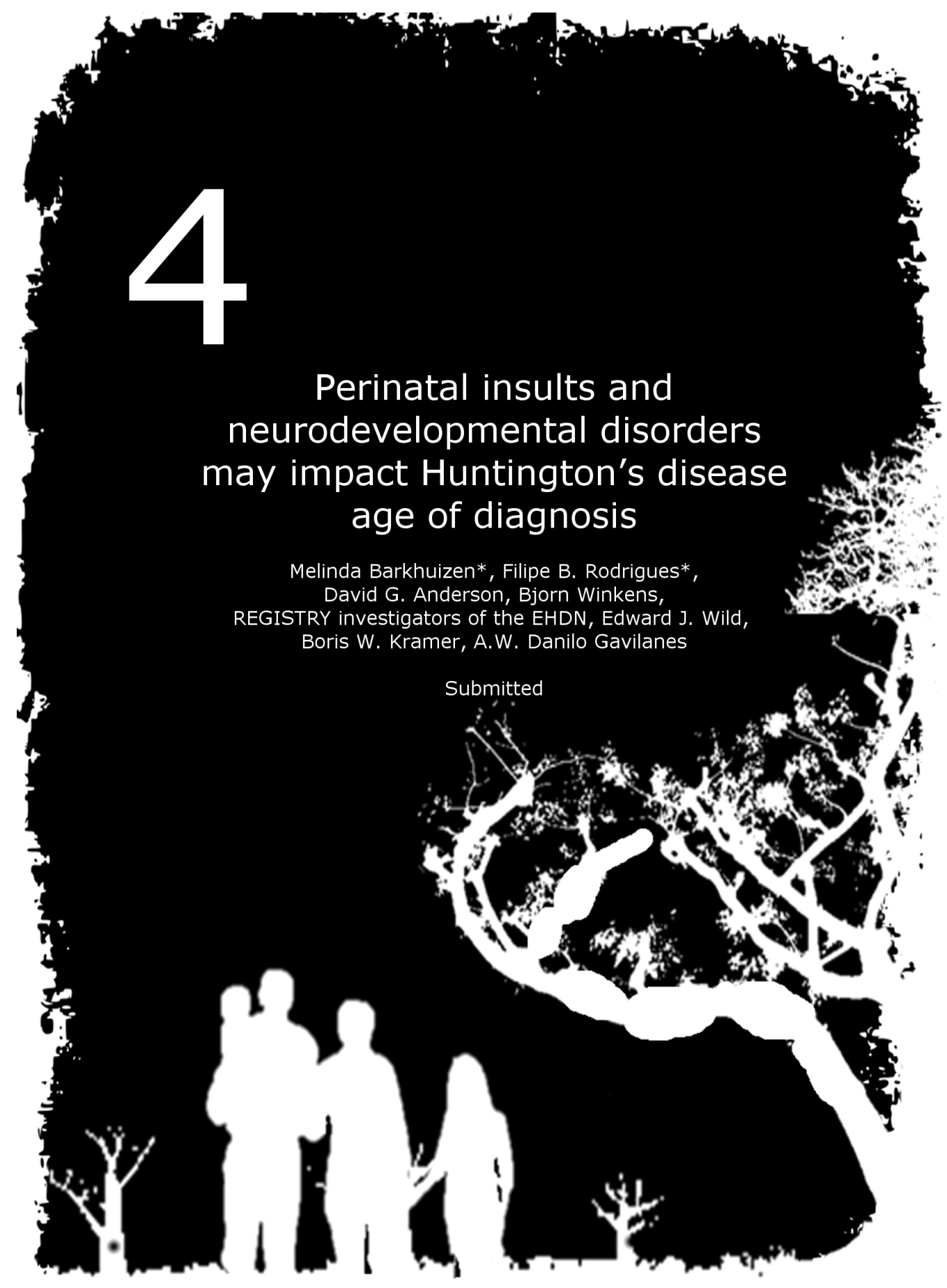




\title{
Perinatal insults and neurodevelopmental dis- orders may impact Huntington's disease age of diagnosis
}

\author{
Melinda Barkhuizen*, Filipe B. Rodrigues*, David G. Anderson, Bjorn Winkens, \\ REGISTRY investigators of the EHDN, Edward J. Wild, Boris W. Kramer, \\ A.W. Danilo Gavilanes \\ * These authors contributed equally \\ Submitted to Journal of Neurology, Neurosurgery and Psychiatry
}

\begin{abstract}
:
Objectives: The objectives of this study were to determine whether early-life factors, such as perinatal insults or neurodevelopmental disorders, are associated with the age of diagnosis of Huntington's disease (HD).

Methods: We used data from 13,856 participants from REGISTRY and Enroll-HD, two large international multicenter observational studies. Diseasefree survival analyses of mutation carriers with an HTT CAG repeat expansion size above and including 36 were computed through Kaplan-Meier estimates of median time until a diagnosis of HD. Comparisons between groups were computed using a Cox proportional hazard survival model adjusted for CAGrepeat expansion length. We also assessed whether the group effect depended on gender and the affected parent.
\end{abstract}

Results: Our results showed that insults in the perinatal period were associated with an earlier median age of diagnosis of 45.00 years (95\%CI: 42.07-47.92) compared to 51.00 years (95\%CI: 50.68-51.31) in the reference group, with a CAG-adjusted hazard ratio of 1.61 (95\%CI: 1.26-2.06). Neurodevelopmental disorders were also associated with an earlier median age of diagnosis than the reference group of 47.00 years (95\% CI: 43.63-50.36) with a CAG-adjusted hazard ratio of 1.41 (95\%CI: 1.15-1.74). These effects did not significantly change with gender or affected parent.

Conclusions: These results, derived from large observational datasets, show that perinatal insults and neurodevelopmental disorders are associated with earlier ages of diagnosis of magnitudes similar to the effects of known genetic modifiers of HD. Given their clear temporal separation, these early events may be causative of earlier HD onset, but further research is needed to 
prove causation.

Keywords: Huntington's disease, Neonatal, Developmental disorders, Epidemiological, Modifier 


\subsection{Introduction:}

Huntington's disease (HD) is a progressive neurodegenerative disease characterized by motor, cognitive and behavioral abnormalities (Rodrigues, Abreu et al. 2017). In Europe, North America, and Australia the overall presence is 5.70 cases per 100,000 (Pringsheim, Wiltshire et al. 2012) and is expected to increase by approximately 15-20\% per decade (Rawlins, Wexler et al. 2016). There is no cure and little evidence to support symptomatic treatment (Rodrigues, Mestre et al. 2017).

The length of the CAG-repeat expansion in the HTT gene is the largest determinant of the age of diagnosis of disease, accounting for approximately $67 \%$ of the overall variation (Gusella and MacDonald 2009). However, there is still substantial variability in the age of diagnosis after controlling for repeat expansion length, which by definition is due to some combination of other genetic or environmental factors or the interaction between genetic and environmental factors. Variation in certain loci has been shown to hasten the onset of disease by up to 6 years or delay it by 1.4-1.6 years (Lee, Wheeler et al. 2015). In a large Venezuelan kindred, as much as $41 \%$ of the age of diagnosis variability in HD families was attributed to environmental factors not shared by family members (Wexler 2004). Identifying environmental modifiers which may be targeted to delay the age-of-onset in pre-manifest gene expansion carriers (GEC) is critical to reducing the burden of HD.

The inheritance of an HD mutation initiates a lifelong pathogenic process, which is eventually followed by symptom onset and clinical diagnosis (Gusella and MacDonald 2009). There is growing evidence that key pathological processes in HD have their origin early in life (Wiatr, Szlachcic et al. 2017). The huntingtin protein (HTT) is important for neurogenesis and neuronal migration (White, Auerbach et al. 1997, Barnat, Le Friec et al. 2017), and a complete loss of HTT function during embryonic development is lethal (Wiatr, Szlachcic et al. 2017). Disruption of HTT function in utero, through reduced expression of normal HTT or expression of mutant poly-CAG HTT, alters the morphology of cortical neurons in adulthood and can cause cortical and striatal degeneration during aging (Arteaga-Bracho, Gulinello et al. 2016, Molero, Arteaga-Bracho et al. 2016).

The aims of this study were to determine whether perinatal insults and neurodevelopmental disorders were modifiers of HD age of diagnosis in two large multicenter international longitudinal cohorts (Orth and Network 2010, Landwehrmeyer, Fitzer-Attas et al. 2016) using survival analysis methodology. 
Perinatal insults have been linked to deficits in functional domains affected in HD, such as cognition, locomotion, behavior or sensory development in approximately $40 \%$ of survivors in the general population (Mwaniki, Atieno et al. 2012). We also examined whether any effects of the insults depended on the gender of the participants or the gender of the parent from which the geneexpansion was inherited since it is possible that the gender of the offspring may influence any relationship with early life events. It is also possible that women affected by Huntington's disease might experience differences in pregnancy, delivery or early childhood development, via biological or social means (e.g. effect of mutant huntingtin on placental development, or differences in perinatal care); and this might exert an independent effect on HD disease-free survival in her offspring, either via the perinatal and neurodevelopmental factors we examine her, or through other routes. We hypothesized that a perinatal insult and/or a neurodevelopmental disorder, and an HD positive genotype could have additive damaging effects, which could manifest as an earlier disease diagnosis.

\subsection{Materials and methods:}

We followed the STROBE guidelines for reporting epidemiological results (Von Elm, Altman et al. 2008) and SAMPL guidelines for reporting statistical findings (Lang and Altman 2014).

\subsubsection{Ethical approval and reporting guidelines:}

This study and its contributing works were performed in accordance with the declaration of Helsinki and approved by the local ethics committees for each study site contributing to REGISTRY (NCT01590589) and Enroll-HD (NCT01574053). All participants gave informed written consent. Participants lacking consenting capacity had consent given on their behalf as requested by country-specific ethical standards. Only data from persons above and including 21 years of age were included. 


\subsubsection{Datasets, study designs, and participants:}

All participants were part of the European Huntington's Disease Network's (EHDN) multicenter, European, prospective observational study - REGISTRY (V2 and V3) (Orth and Network 2010); or of Enroll-HD (2016 release) (Landwehrmeyer, Fitzer-Attas et al. 2016). 14,893 participants from 165 study sites in 21 European countries were enrolled in REGISTRY between 2004 and 2016. Enroll-HD succeeded REGISTRY and also included participants from North America, Latin America, and Australasia enrolled between 2012 and 2016. The Enroll-HD 2016 release contained the data of 8,714 participants, including 3,598 participants previously enrolled in REGISTRY. These longitudinal cohorts include manifest and pre-manifest GEC, as well as healthy controls and individuals at risk of HD. For our analysis, we only included GEC with: a CAG-repeat expansion length above and including 36 repeats on the major allele; an age at diagnosis or an age at last visit above and including 21 years; and available co-morbidities data. We further removed duplicate records from the Enroll-HD participants who were also enrolled in REGISTRY. This limited the number of participants to 7,686 manifest GEC and 2,069 pre-manifest GEC from REGISTRY, and 2,892 manifest and 1,209 pre-manifest GEC from Enroll-HD. Since the variables of interest were similar between studies, as were the study designs, the two datasets were combined into one large data set after excluding duplicate records. The final data set for calculating the influence of age at diagnosis included the data of 10,578 manifest and 3,278 pre-manifest GECs. For a secondary analysis on the influence of affected parent on the model, data were available for 7,271 manifest and 3,207 pre-manifest GECs. The amounts of participants included in each stage are shown in figure 4.1.

\subsubsection{Identification of perinatal complications and neurodevel- opmental disorders:}

Two perinatal investigators (M.B., A.G.) examined all the comorbidities listed in each database to identify perinatal insults and neurodevelopmental disorders. Perinatal insults (MeSH ID: D054238) were defined as insults which likely occurred between 28 weeks of gestation to 28 days after birth. Where recorded, the age at the event and ICD10 codes were used to describe events that occurred during the neonatal period, as opposed to complications of pregnancy described in the records of the mother. Dates of the adverse events were not recorded for all participants and where recorded, it was sometimes possible to narrow the insult down to the first year of life but not the first 28 days after birth. Conditions of this kind that were additionally listed as 'intrauterine', 'perinatal' or 'neonatal' were considered as having occurred within the perinatal 


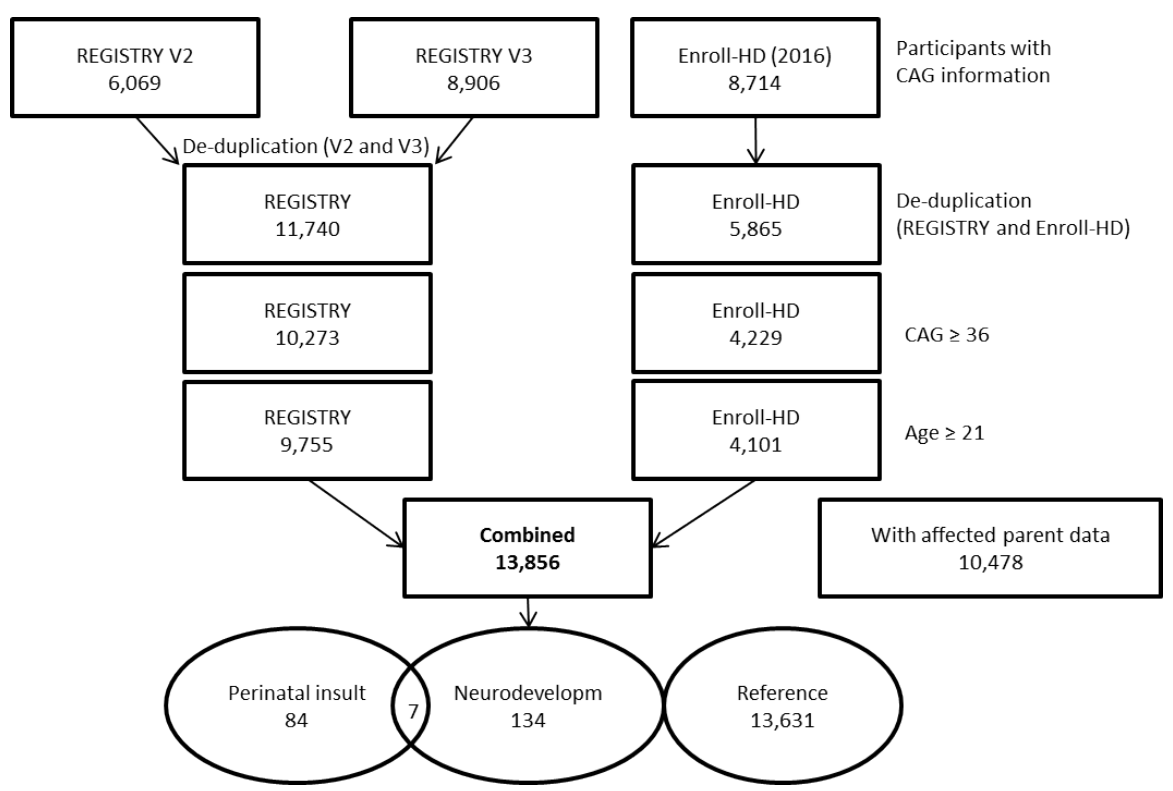

Figure 4.1: Flow chart of included participants and exclusions. Neurodevelopm, neurodevelopmental disorders

time-frame.

For neurodevelopmental disorders, the co-morbidities records were screened for neurodevelopmental disorders included in the DSM-5 and ICD10 classification. This list includes conditions such as neurocognitive disorders, communication and language deficits, autism and attention deficit hyperactivity disorder. The age-limit for neurodevelopmental disorders was set at 20 years and attention deficits above this age and psychiatric conditions, such as schizophrenia were excluded. We further excluded tics and Tourette's syndrome as these may mimic the symptoms of HD. The list was supplemented with conditions listed in reviews on the neurodevelopmental outcomes of perinatal insults (Mwaniki, Atieno et al. 2012, Volpe 2012). Non-specific disorders that could also have an adulthood onset, such as seizures, visual loss, and hearing loss were excluded, with the exception of the pediatric visual disorder strabismus (Hoyt and Taylor 2012). 


\subsubsection{Statistical analysis:}

To determine participants' age of diagnosis, we used the Kaplan-Meier product limit method (median and 95\% confidence interval [95\% CI]). A single estimate of the age of diagnosis in years was derived for each group of participants for each of the databases. For pre-manifest GECs, we used the latest visit date in their profile as an age of diagnosis-free survival. The primary analysis was done with the merged and de-duplicated dataset, and secondary sensitivity analyses were done with REGISTRY and Enroll-HD datasets independently.

The effects of perinatal insults and neurodevelopmental disorders on HD age of diagnosis were adjusted for CAG-repeat expansion length on the major allele through a Cox proportional hazards model. The assumption for the constant hazard ratios (HR) was tested with Schoenfeld residuals and with time-dependent covariates. The overall proportional hazard assumption was not violated for analyzing time until diagnosis by the group, as the time-dependent covariates $(\mathrm{p}=0.385)$, as well as Schoenfeld residuals, were not significant (all $\mathrm{p}=0.188$ ). Participants were grouped into three groups: perinatal insults, neurodevelopmental disorders, and the reference group consisting of the remaining participants (Table 4.1 and 4.2). HR and 95\%CI were generated for each of the groups, compared to the reference group.

Additionally, we investigated whether the effect seen per group depended on the gender of the participant or the gender of the parent from whom the mutation was inherited. The merged cohort was used for this analysis. The participant gender info was available for all 10,578 manifest and 3,278 pre-manifest GECs, however, affected parent data was only available for 7,271 manifest and 3,207 pre-manifest GECs. Therefore, we first performed a multiple imputation (MI) method for the missing values of an affected parent using all the variables included in the Cox regression model as a predictor. After the MI, where the maximum number of iterations was set to 20,30 complete data sets were created and Cox regression analysis was applied to each dataset and then pooled. To assess effect modification we calculated interaction terms between group and gender, and between the group and affected parent in the model of the CAG adjusted HR. If the interaction term with gender and/or affected parent was statistically significant, we expressed the group effect for each level of gender and/or affected parent with the corresponding CAG-adjusted HR and $95 \% \mathrm{CI}$. As a sensitivity analysis, we also performed the complete case analysis, i.e. applying the same model to only the participants with complete information on both parent and sex. We compared the pooled results after MI to the results obtained from the complete case analysis. Two-sided p-values 

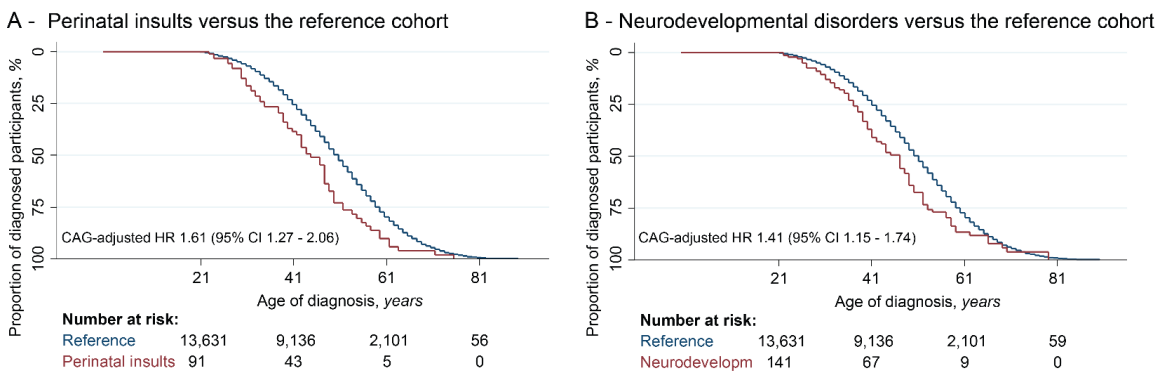

Figure 4.2: Kaplan-Meier survival curves of the age of diagnosis for the merged cohort. A Participants with perinatal insults versus the "reference" group; B Participants with neurodevelopmental disorders versus the "reference" group. 95\% CI, 95\% confidence interval; HR, hazard ratio; Neurodevelopm, neurodevelopmental disorders.

below 0.05 were considered statistically significant. Statistical analyses were performed with IBM SPSS Statistics for Windows (Version 24.0, Armonk, NY).

\subsection{Results:}

4.3.1 Description of comorbidities included: In the combined cohort, there were 91 participants with perinatal insults and 141 participants with neurodevelopmental disorders included. Seven cases had both a perinatal insult and a neurodevelopmental disorder. The comorbidities included in this study are listed in table 4.1

\subsubsection{Influence of perinatal insults and neurodevelopmental dis- orders in disease-free survival:}

From the merged cohort, 13,856 GECs were included in our survival analysis, of which 10,578 had manifest HD and 3,278 were pre-manifest carriers. The Kaplan Meier survival plot (figure 4.2) showed that both perinatal insults (PI) and neurodevelopmental disorders (ND) had a reduced time until an HD diagnosis (unadjusted HR's: 1.607 [95\% CI: 1.259-2.051], 1.365 [95\% CI: 1.112-1.676], respectively; CAG-adjusted HR's: 1.614 [95\% CI: 1.265-2.061], 1.414 [95\% CI: 1.152-1.737],respectively).

The overall time until diagnosis, characteristics and hazard ratios for each group are shown in table 4.2. The secondary sensitivity analysis from the REGISTRY cohort confirmed these results for both the perinatal insults group and the neurodevelopmental disorders group (CAG-adjusted HR 1.64 [95\% CI: 
Table 4.1: Description of the comorbidities included from the REGISTRY and EnrollHD cohorts, divided into perinatal insults and neurodevelopmental disorders. $\mathrm{N}$, the number of comorbidities $\%$, percentage of comorbidities.

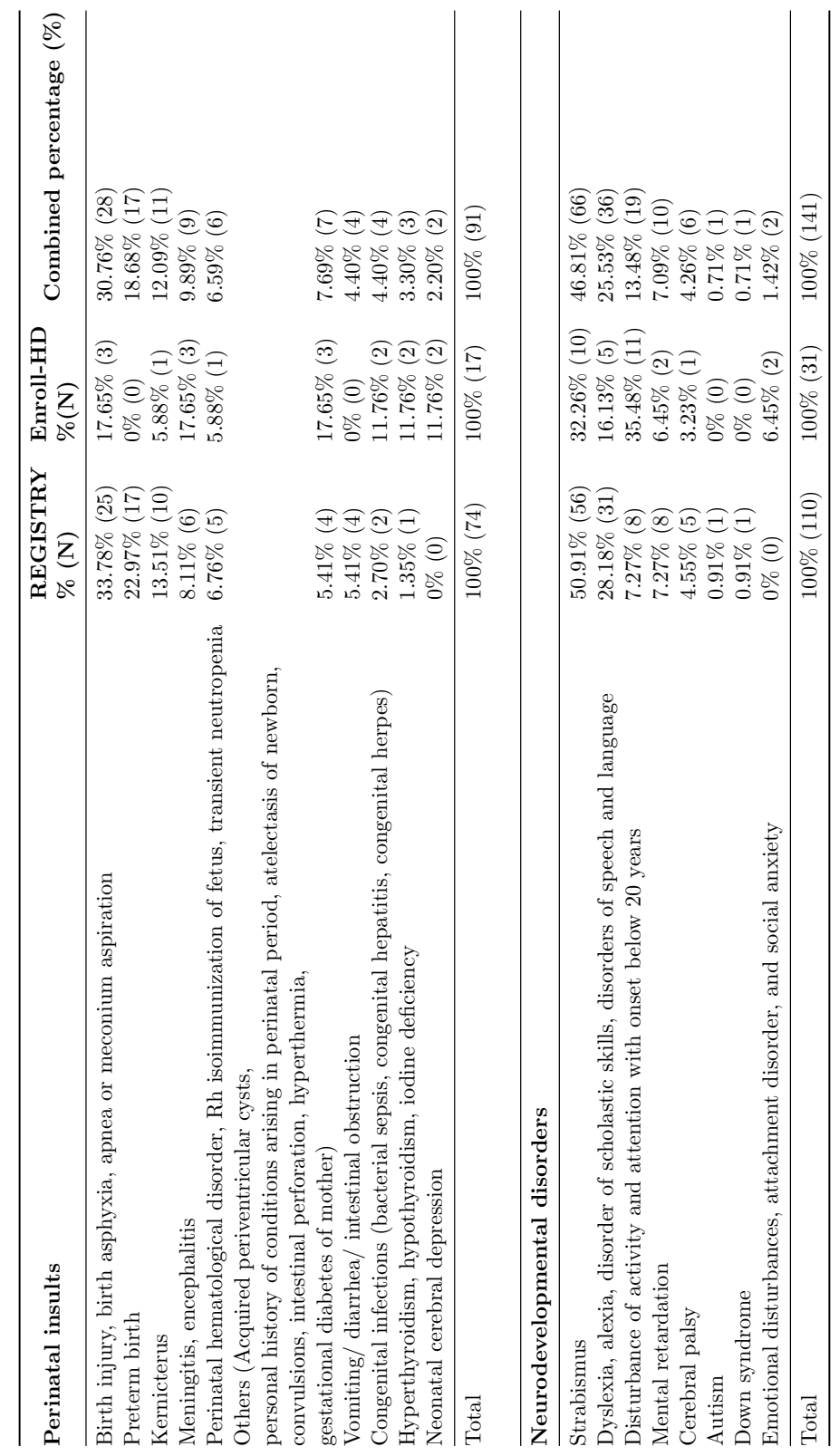


1.26-2.15], HR 1.61 [95\% CI: 1.28-2.02], respectively). The Enroll-HD cohort showed non-statistically significant results (CAG-adjusted HR 1.45 [95\% CI: 0.80-2.63], HR: 0.24 [95\% CI: 0.15-0.39], respectively).

\subsubsection{The effect of gender and affected parent on disease-free survival per group:}

The overall time until diagnosis of HD per gender and affected parent is shown in Table 4.3. The interactions between group and gender, or group and affected parent were not statistically significant for either perinatal insults or neurodevelopmental disorders (see Table 4.3 for further details).

\subsection{Discussion and conclusions:}

Our results show that perinatal insults and neurodevelopmental disorders were associated with earlier age of diagnosis of HD, with an observed unadjusted difference of 4-6 years. The group differences in age-of-diagnosis were not significantly modified by either gender or affected parent. These differences are substantial since the most robust recently-described genetic modifiers, rs148491145 on chromosome 14 and rs146353869 on chromosome 15, altered the onset of disease by 3.2 and 6.1 years, respectively (Lee, Wheeler et al. 2015). One possible explanation is that these early-life events speed up the onset of HD through diminishing neural reserve, accelerating neuropathology or altering neurodevelopment in a way that predisposes to earlier onset. Despite the clear temporal separation between perinatal and developmental problems and subsequent HD onset, we were unable to assess dose response, specificity or experimental evidence from other biological systems, to confirm causality according to the Bradford Hill's criteria for causality in a biological system (Hill 1965). Further research on these aspects is needed to establish causation.

The most common insults in the cohorts were direct birth injuries/asphyxia, followed by preterm birth, neonatal/congenital infections and kernicterus. We only included sepsis, meningitis or congenital infections of participants where it could be ascertained that the insult did indeed occur in the first year of life. Asphyxia-related neonatal encephalopathy occurred in $0.85 \%$ of live births globally in 2010 (Lee, Kozuki et al. 2013). In the general population, surviving infants often have a spectrum of neurological impairment, ranging from normal functioning to severe neurological disabilities; like cerebral palsy, attention deficit hyperactivity disorder (ADHD), autism, congenital hearing loss and neonatal seizures (Odd and Whitelaw 2013). Preterm birth, defined as birth 
Table 4.2: Characteristics of the merged cohort, the REGISTRY cohort, and the Enroll-HD cohort, divided by the group of participants, including sample sizes, gender ratio, the median age of diagnosis in years, median CAG length, and percentage and number of Caucasians, Europeans and North Americans. 95\%CI, 95\% confidence interval; HR, hazard ratio; IQR, interquartile range; N, the number of participants; \%, the percentage of participants.

\begin{tabular}{|c|c|c|c|}
\hline & Merged cohort & REGISTRY & Enroll-HD \\
\hline \multicolumn{4}{|l|}{ Reference cohort } \\
\hline N (N manifest) & $13,631(10,428)$ & $9,578(7,566)$ & $4,053(2,862)$ \\
\hline$\%$ male & $46.63 \%$ & $46.82 \%$ & $46.57 \%$ \\
\hline Median CAG (IQR) & $43(41-45)$ & $43(41-45)$ & $43(41-45)$ \\
\hline Median age of diagnosis $(95 \% \mathrm{CI})$ & $51.00(50.68-51.32)$ & $50.00(49.63-50.37)$ & $52.00(51.43-52.57)$ \\
\hline$\%$ Caucasian $(\mathrm{N})$ & $95.83 \%(13,062)$ & $97.52 \%(9,340)$ & $90.20 \%(2,656)$ \\
\hline$\%$ Europe $(\mathrm{N})$ & $81.90 \%(11,164)$ & $100 \%(9,578)$ & $39.13 \%(1,586)$ \\
\hline$\%$ North-America (N) & $16.00 \%(2,181)$ & $0 \%(0)$ & $53.81 \%(2,181)$ \\
\hline \multicolumn{4}{|l|}{ Perinatal insults } \\
\hline $\mathbf{N}$ (N manifest HD) & $91(65)$ & $74(54)$ & $17(11)$ \\
\hline$\%$ male & $45.05 \%$ & $47.30 \%$ & $35.29 \%$ \\
\hline Median CAG (IQR) & $44(42-47)$ & $44(42-47)$ & $43(41-44)$ \\
\hline Median age of diagnosis $(95 \% \mathrm{CI})$ & $45.00(42.07-47.93)$ & $43.00(39.84-46.16)$ & $49.00(43.57-54.43)$ \\
\hline$\%$ Caucasian $(\mathrm{N})$ & $97.80 \%(89)$ & $97.30 \%(72)$ & $100 \%(17)$ \\
\hline$\%$ Europe $(\mathrm{N})$ & $90.11 \%(82)$ & $100 \%(74)$ & $47.06 \%(8)$ \\
\hline$\%$ North-America (N) & $9.89 \%(9)$ & $0 \%(0)$ & $52.94 \%(9)$ \\
\hline CAG-adjusted HR (95\%CI) & $1.61(1.27-2.06)$ & $1.64(1.26-2.15)$ & $1.45(0.80-2.63)$ \\
\hline \multicolumn{4}{|l|}{ Neurodevelopmental disorders } \\
\hline $\mathbf{N}(\mathbf{N}$ manifest HD) & $141(92)$ & $110(73)$ & $31(19)$ \\
\hline$\%$ male & $48.93 \%$ & $52.72 \%$ & $35.48 \%$ \\
\hline Median CAG (IQR) & $44(42-46)$ & $44(42-46)$ & $42(40-44)$ \\
\hline Median age of diagnosis $(95 \% \mathrm{CI})$ & $47.00(43.63-50.37)$ & $45.00(41.36-48.64)$ & $53.00(47.30-58.70)$ \\
\hline$\%$ Caucasian $(\mathrm{N})$ & $96.45 \%(136)$ & $98.18 \%(108)$ & $90.32 \%(28)$ \\
\hline$\%$ Europe $(\mathrm{N})$ & $83.69 \%(118)$ & $100 \%(110)$ & $25.81 \%(8)$ \\
\hline$\%$ North-America (N) & $16.31 \%(23)$ & $0 \%(0)$ & $74.19 \%(23)$ \\
\hline CAG-adjusted HR (95\%CI) & $1.41(1.15-1.74)$ & $1.61(1.28-2.02)$ & $0.24(0.15-0.39)$ \\
\hline
\end{tabular}


Table 4.3: Survival differences by gender and affected parent per group, including sample sizes, gender ratio, the median age of diagnosis in years, and median CAG length. 95\% CI, 95\% confidence interval; IQR, interquartile range; $\mathrm{N}$, the number of participants.

\begin{tabular}{|c|c|c|c|}
\hline & Reference & Perinatal insults & Neurodevelopmental disorders \\
\hline \multicolumn{4}{|c|}{ Effect of gender of participant on overall survival per group } \\
\hline \multicolumn{4}{|l|}{ Males } \\
\hline $\mathrm{N}$ (N manifest HD) & $6356(5093)$ & $41(30)$ & $69(46)$ \\
\hline Median age of diagnosis $(95 \% \mathrm{CI})$ & $50.00(49.57-50.44)$ & $43.00(40.45-45.55)$ & $48.00(43.76-52.24)$ \\
\hline Median CAG (IQR) & $43(4)$ & $44(4.5)$ & $43(5)$ \\
\hline \multicolumn{4}{|l|}{ Females } \\
\hline $\mathrm{N}$ (N manifest HD) & $7275(5335)$ & $50(35)$ & $72(46)$ \\
\hline Median age of diagnosis $(95 \% \mathrm{CI})$ & $51.00(50.54-51.55)$ & $47.00(44.26-49.75)$ & $47.000(43.09-50.91)$ \\
\hline Median CAG (IQR) & $43(4)$ & $43(5.25)$ & $44(4)$ \\
\hline \multicolumn{4}{|l|}{ Comparison between genders } \\
\hline $\mathrm{p}$-value & - & 0.537 & 0.799 \\
\hline \multicolumn{4}{|c|}{ Effect of gender of affected parent on overall survival per group } \\
\hline \multicolumn{4}{|l|}{ Affected father } \\
\hline $\mathrm{N}$ (N manifest HD) & $4747(3476)$ & $29(22)$ & $55(34)$ \\
\hline Median age of diagnosis $(95 \% \mathrm{CI})$ & $49.00(48.46-49.54)$ & $43.00(41.50-44.50)$ & $42.00(34.67-49.33)$ \\
\hline Median CAG (IQR) & $43(4)$ & $44(6)$ & $44(5)$ \\
\hline \multicolumn{4}{|l|}{ Affected mother } \\
\hline $\mathrm{N}$ (N manifest $\mathrm{HD})$ & $5359(3675)$ & $45(27)$ & $63(37)$ \\
\hline Median age of diagnosis $(95 \% \mathrm{CI})$ & $51.00(50.47-51.53)$ & $48.00(41.02-54.98)$ & $47.00(44.11-49.89)$ \\
\hline Median CAG (IQR) & $43(4)$ & $44(5)$ & $43(5)$ \\
\hline \multicolumn{4}{|l|}{ Comparison between affected parent } \\
\hline p-value (complete case analysis) & - & 0.699 & 0.977 \\
\hline p-value (multiple imputation) & - & 0.791 & 0.874 \\
\hline
\end{tabular}

before 37 weeks of gestation, occurred in $11.1 \%$ of live births globally in 2010 (Blencowe, Cousens et al. 2013). Preterm birth is commonly associated with cognitive, behavioral, attentional, or socialization deficits and occasionally with major motor deficits in the general population. The majority of the disability burden consists of infants born at a very low gestational age, before 28 weeks (Volpe 2009c). The risk of severe medical disabilities decreases sharply with increasing gestational age. Survivors of preterm birth without serious medical disabilities have a slightly lower chance of achieving higher education and holding well-paying jobs (Moster, Lie et al. 2008). These survivors also have a higher mortality risk in adulthood due to a multitude of health risks; including increased rates of diabetes, metabolic syndrome, neuropsychiatric disorders, respiratory, cardiovascular and kidney diseases (Crump, Sundquist et al. 2016). The prevalence of direct birth trauma $(0.20 \%)$ and preterm birth $(0.12 \%)$ in these HD cohorts were much lower than expected from their prevalence in the general population. This likely reflected the difficulties of retrospectively assessing events which occurred several years before enrollment. Despite our use of a robust survival analysis, we could not eliminate the effects of this apparent recall bias. One limitation of our approach was that the perinatal insults and neurodevelopmental disorders groups were based on conditions affecting several divergent functional domains since these disorders are rela- 
tively rare in the general population, and not all persons with perinatal insults develop a neurodevelopmental disorder. A further limitation was that the effect of neurodevelopmental disorders was not replicated in the Enroll-HD cohort, likely due to an insufficient sample size and divergent participant characteristics.

Overall, the most common adverse outcomes reported after perinatal insults in the general population were: learning difficulties, cognitive deficits or a developmental delay in approximately $60 \%$ of cases; cerebral palsy $(21 \%)$ hearing impairment (20\%); visual impairment (18\%) or behavioral problems (11\%) (Mwaniki, Atieno et al. 2012). In our neurodevelopmental disorders group, we assessed ADHD, autism spectrum disorders, scholastic difficulties, cognitive impairment and cerebral palsy and the common childhood visual disorder strabismus.

In the HD cohorts, strabismus, disturbances in attention or activity, and dyslexia or alexia were the most common adverse neurodevelopmental outcomes. Strabismus affects 2-3\% of children in the general population (Friedman, Repka et al. 2009). It has a heritable component, as well as obstetric causes. Preterm birth, low birth weight, or a low Apgar score after birth increases the risk of strabismus in the general population (Maconachie, Gottlob et al. 2013). Similarly, ADHD and dyslexia could be linked to genetics (Grigorenko 2005, Schachar 2014), environmental causes, maternal factors or birth complications (Thapar, Cooper et al. 2013, Smith, Schmidt-Kastner et al. 2016). ADHD affects 5.3\%$7.2 \%$ of children below 18 years of the general population (Polanczyk, de Lima et al. 2007, Thomas, Sanders et al. 2015). Developmental dyslexia affects approximately 7\% of the general population (Peterson and Pennington 2012). The prevalence of these disorders among HD GECs were $0.48 \%$ for strabismus, $0.26 \%$ for dyslexia or alexia and $0.14 \%$ for childhood-onset attention deficits. These percentages were somewhat lower than in the general population. This could be due to incomplete medical histories recorded in large longitudinal cohorts, or because HD participants with additional comorbidities may be less inclined to participate in research.

There was minimal overlap between our perinatal insults group and our neurodevelopmental disorder group. This may be due to incomplete reporting of neonatal insults in the neurodevelopmental group or due to the multifactorial causes of neurodevelopmental disorders.

Hypothetically, numerous possible mechanisms could be argued to justify a potential causative link between perinatal and developmental problems and 
earlier onset of HD. Epigenetic alterations after perinatal insults could increase the vulnerability of the brain to subsequent insults, like mutant HTT, after a long latent phase (Lahiri, Maloney et al. 2009). Several general mechanisms of neurotoxicity and neurodegeneration, such as excitotoxicity, oxidative stress, and mitochondrial dysfunction are shared between HD and encephalopathy due to perinatal insults. The striatum is particularly vulnerable to these insults (Ehrlich 2012, Barkhuizen, Van den Hove et al. 2017). The pathology of both diseases has also been linked to an over-activation of the DNA repair pathways. Perinatal asphyxia compromises the integrity of the genome, which triggers the activation of sentinel proteins that maintain genome integrity (Morales, Bustamante et al. 2011). In HD, large somatic expansions in the striatum and cortex are associated with a decreased age of disease onset. These expansions are thought to occur during the process of DNA mismatch repair(Bettencourt, Hensman-Moss et al. 2016). Notwithstanding that our results cannot establish causation, these possibilities warrant exploration, perhaps in experimental models of HD.

In conclusion, this work shows that perinatal and neurodevelopmental insults may have an impact on age at diagnosis of HD, with an effect comparable to that seen with known genetic modifiers. Further research is needed into the basis and mechanisms of this association. These observations emphasize the far-reaching impact of early-life events and the need to study and minimize them.

\subsection{Acknowledgements:}

The data for this study was kindly provided by two longitudinal Huntington's disease observational studies: REGISTRY and Enroll-HD. These studies are intended to accelerate progress towards therapeutics and are sponsored by CHDI Foundation, a nonprofit biomedical research organization exclusively dedicated to developing therapeutics for HD. REGISTRY and Enroll-HD would not be possible without the vital contribution of the research participants and their families. The funders of the study had no influence on the study design or results reported. 



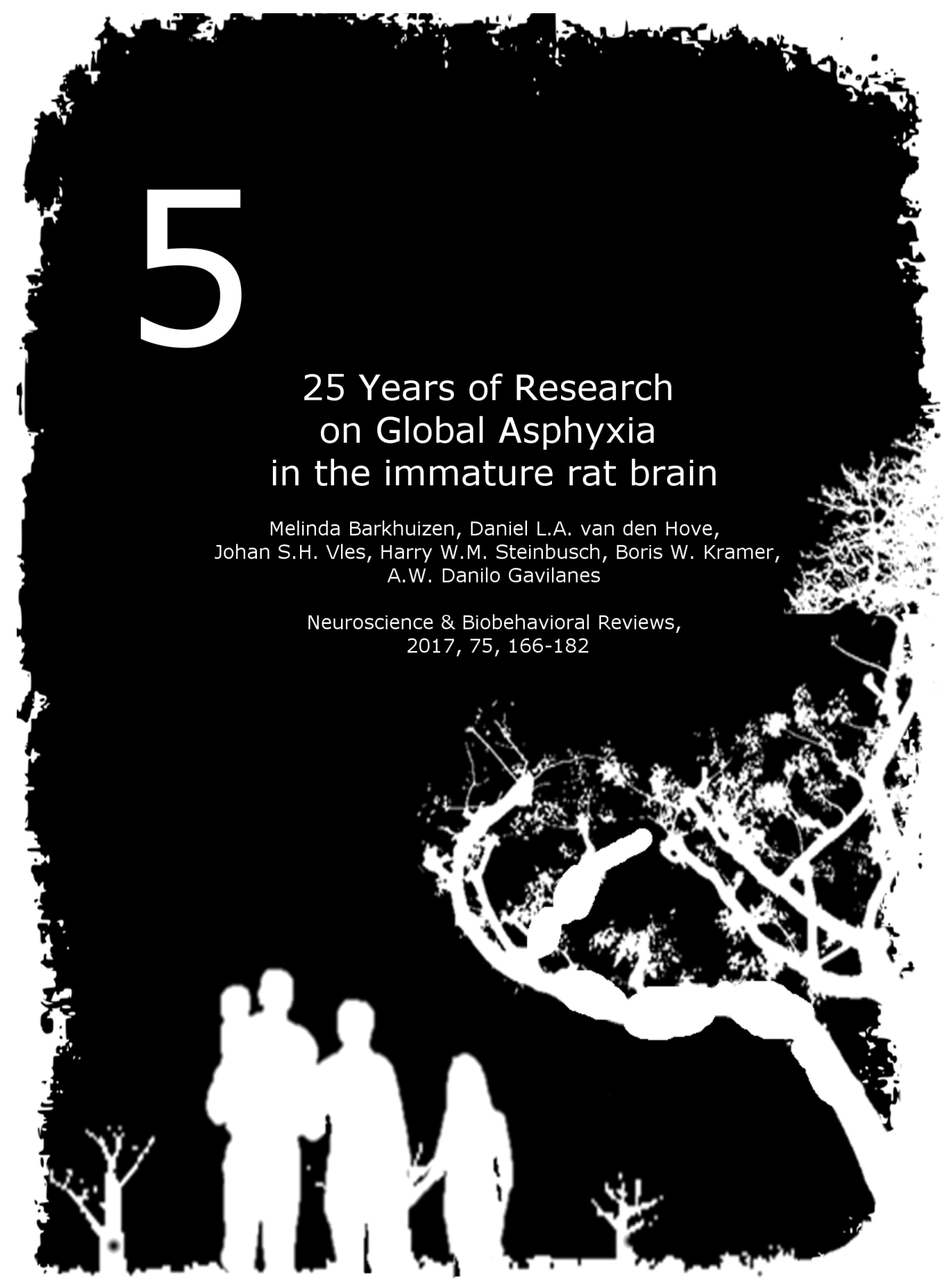




\title{
25 Years of research on global asphyxia in the immature rat brain
}

Melinda Barkhuizen, Daniel L.A. van den Hove, Johan S.H. Vles, Harry W.M. Steinbusch, Boris W. Kramer, A.W. Danilo Gavilanes

Neuroscience \& Biobehavioral Reviews, 2017, 75, 166-182

\begin{abstract}
:
Hypoxic-ischemic encephalopathy remains a common cause of brain damage in neonates. Preterm infants have additional complications as prematurity by itself increases the risk of encephalopathy. Currently, therapy for this subset of asphyxiated infants is limited to supportive care. There is an urgent need for therapies in preterm infants - and for representative animal models for preclinical drug development. In 1991, a novel rodent model of global asphyxia in the preterm infant was developed in Sweden. This method was based on the induction of asphyxia during the birth processes itself by submerging pups, still in the uterine horns, in a water bath followed by C-section. This insult occurs at a time-point when the rodent brain maturity resembles the brain of a 22-32 week old human fetus. This model has developed over the past 25 years as an established model of perinatal global asphyxia in the early preterm brain. Here we summarize the knowledge gained on the short- and long-term neuropathological and behavioral effects of asphyxia on the premature central nervous system.
\end{abstract}

Keywords: Hypoxic-ischemia, encephalopathy, preterm infant, submersion, functional outcome

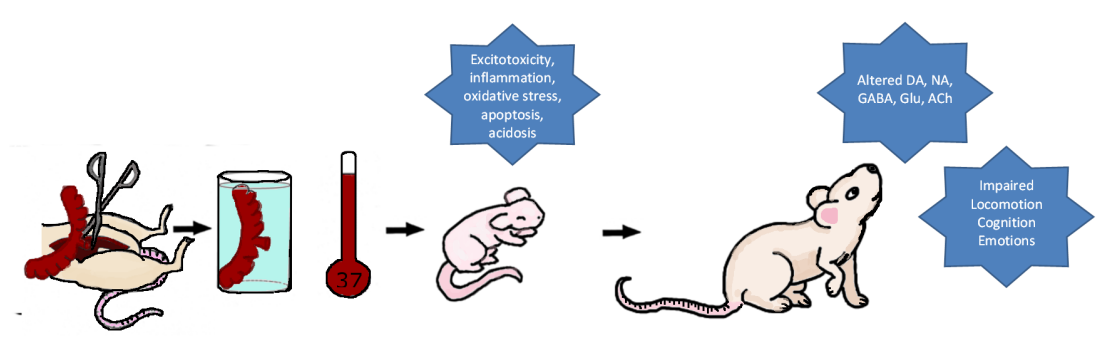

Figure 5.1: Graphical abstract (adapted from (Sparnaaij, Chedraui et al. 2016). 


\subsection{Introduction:}

\subsubsection{Perinatal asphyxia in the preterm infant:}

Perinatal asphyxia (PA) occurs when blood gas exchange between mother and fetus is impaired. This leads to progressive hypoxia, hypercapnia and ischemia with significant metabolic acidosis. Metabolically privileged organs like the brain, heart, kidneys and liver are especially sensitive. PA can cause hypoxic ischemic encephalopathy (HIE) which affects 2.5 per 1000 live born term infants (Graham, Ruis et al. 2008) which translates to 4-9 million infants globally (Black, Cousens et al. 2010, Wachtel and Hendricks-Muñoz 2011). The long-term neurological outcome after HIE varies, from normal neurocognitive functioning after mild asphyxia to cerebral palsy, epilepsy, cognitive, behavioral or memory problems in severe cases (Armstrong-Wells, Bernard et al. 2010). These abnormalities are due to structural damage to the central nervous system (CNS). The brain areas most susceptible to damage vary according to the maturity of the fetus at the time of PA. In full-term fetuses, severe PA commonly damages the thalamus, peri-rolandic cerebral cortex, basal ganglia, corpus striatum and brainstem due to excessive glutamatergic stimulation of the cortical-thalamic and the cortical-striatum connections (Johnston 2005, Cabaj, Bekiesińska-Figatowska et al. 2012).

In the preterm fetus, patterns of injury are more complex. Prematurity by itself increases the risk of encephalopathy, without the added asphyctic insult. Factors like the degree of maturity of the brain, selective ischemic vulnerability of developing neuron subpopulations, the severity of the insult and the characteristics of the reperfusion after hypoxic-ischemia determine the severity of the damage after PA (Alvarez-Diaz, Hilario et al. 2007, Volpe 2009a, Volpe 2009c). In this population, the most common site of injury is the developing oligodendrocytes within the sub-cortical white matter (periventricular leukomalacia). Associated grey-matter injury to the basal ganglia, thalamus, basis pontis and brain stem injury has been reported in infants with periventricular leukomalacia. Common long-term complications in preterm survivors of PA include death within the first 2 years and cerebral palsy ranging from mild impairment to quadriplegia (Pierson, Folkerth et al. 2007, Logitharajah, Rutherford et al. 2009, Cabaj, Bekiesińska-Figatowska et al. 2012).

While preterm birth before 37 weeks of gestation occurs in 5-8\% of all pregnancies, very low gestational age (VLGA) birth, before 32 weeks of gestation, occurs in about $1 \%$ of singletons and $9 \%$ of twin pregnancies (Schaaf, Mol et al. 2011). In cohorts of preterm infants with multifactorial encephalopathy, behavioral, cognitive, attention or social deficits have been reported in 25-50\% 
of cases and 5-10\% had major motor impairments. A large portion of this disability burden was made up by VLGA infants, due to improved survival rates with modern medical care (Volpe 2009a). Among VLGA infants, the mortality rate ranges from $7.3-21.4 \%$ at 30 days and $9.0-22.7 \%$ at 1 year after birth (Numerato, Fattore et al. 2015).

Several critical neuronal networks develop towards the end of pregnancy, and are thus especially vulnerable in the preterm brain. The population of sub-plate neurons in the dorsal telencephalic ventricular zone reaches its peak size at 24-32 weeks of gestation. The development of these neurons is intertwined with development of the cerebral cortex and the thalamus. During 25-27 weeks of gestation, neuronal progenitors from the subventricular zone are actively producing neurons for the deep cortical layers. The cerebellum also rapidly develops during the last half of human gestation. Any impairment of neurogenesis in these areas, due to PA or other insults, is likely to cause long-term functional consequences in survivors (Volpe 2009b, Volpe 2009c).

Currently, therapeutic hypothermia is the only routinely used clinical intervention for full-term infants with PA. This intervention reduces death and disability, but it does not provide complete protection (Jacobs, Berg et al. 2013, Azzopardi, Strohm et al. 2014). For preterm infants, there are currently no specific therapeutic options available. A small pilot trial with selective head cooling in infants born at 32-35 weeks concluded that there is no evidence that it is safe or beneficial in preterm infants yet (Walsh, Butler et al. 2015). Therefore, it is an urgent need for new therapeutics in PA and for clinically relevant models to test candidates for single-agents or combination therapies in. In 1991, Bjelke et. al. developed a rat model of global PA in the VLGA infant by submersion of the uterine horns during birth on embryonic day 21-22 (Bjelke, Andersson et al. 1991). This review will give an overview of all the knowledge gained on brain function in this model over the past 25 years.

\subsubsection{The perinatal submersion model:}

Numerous animal models had been established to study HIE. In the perinatalsubmersion model, discussed here, PA is induced at day 21-22 of embryonic development in a pregnant rat (Rattus norvegicus). After natural birth of the first pup, the dam is either anesthetized followed by a neck dislocation or rapidly decapitated. A hysterectomy is performed and the uterus horns, still containing the fetuses, are placed in a water bath filled with saline at $37^{\circ} \mathrm{C}$ for a specified duration (0-22 minutes). The pups are then removed from the 
uterine horns for delivery and resuscitated by cleaning the nose and mouth from fluid and amniotic tissue. Pulmonary breathing is stimulated by touching the surface of the nose and mouth, as well as by pressing the thorax (Bjelke, Andersson et al. 1991, Dell'Anna, Chen et al. 1997). This model has been adjusted by varying the duration of submersion and the temperature of the saline solution. It has also been adapted to other rodent species, including the spiny mouse (Acomys cahirinus) (Ireland, Dickinson et al. 2008, Fleiss, Coleman et al. 2011, Fleiss, Parkington et al. 2012) and the guinea pig (Cavia porcellus) (Vaillancourt and Boksa 2000, Bernert, Hoeger et al. 2003). In this review, we focus on studies conducted in the rat.

\subsubsection{Factors to consider when using the perinatal-submersion model:}

\subsubsection{The maturity of the rodent brain at the time of in- sult:}

The susceptibility of brain regions to PA partially depends on their developmental stage during the time of the insult. Disturbances to regions which are rapidly growing at the time of insult can have profound long-term consequences. In humans the majority of brain development occurs prenatally, with a peak in brain growth around birth. In rats the majority of brain development occurs postnatally, with the growth spurt peaking around postnatal day P7. The rat cortex reaches around $90 \%$ of its adult weight by P20, while in humans this occurs around 2-3 years of age. Key rodent neurodevelopmental processes that occur shortly after birth (from P1-3) take place during week 23-32 of human fetal brain development. These processes include progression in the oligodendrocyte maturation state to mitotically active pre-oligodendrocytes, immune system development and establishment of the blood-brain-barrier. The brain maturation state explains the predominance of white-matter injury seen in preterm infants (Semple, Blomgren et al. 2013). Thus, the perinatalsubmersion model investigates brain damage in the rodent equivalent of VLGA infants. 


\subsubsection{The degree of asphyxia and temperature:}

The extent of the damage seen in this model is dependent on the length of asphyxia, the temperature of the saline solution and the sex of the rodents. At a saline temperature of $37^{\circ} \mathrm{C}$, submersion of the uterine horns for $10 \mathrm{~min}$ caused slight PA, 15 min caused moderate PA, 19 min caused sub-severe PA and 20 min cause severe PA. Durations of submersion exceeding 21 min caused non-recovery of almost all the pups (Loidl, Herrera-Marschitz et al. 1994, Chen, Engidawork et al. 1997, Chen, Hillefors-Berglund et al. 1997, Dell'Anna, Chen et al. 1997).

Shortly after mild-moderate PA increased neuronal activity has been reported, whilst severe PA suppresses neuronal activity (Tang, Ringstedt et al. 2000). By lowering the saline temperature to $15^{\circ} \mathrm{C}$, the uterine horns can be submerged for more than 2 hours and many of the damaging effects of PA can be prevented (Loidl, Gavilanes et al. 2000).

The mortality rates rise according to the duration of asphyxia. The majority of pups survive 15-16 minutes of PA. After sub-severe to severe PA (for 19-20 minutes), mortality rates of 30-70\% have been reported across numerous studies. This increased to $97 \%$ after 21-22 minutes of asphyxia at a saline temperature of $37^{\circ} \mathrm{C}$. If asphyxia is induced at $30^{\circ} \mathrm{C}$, the majority of pups survive 46 minutes of submersion (Herrera-Marschitz, Loidl et al. 1993, Loidl, De Vente et al. 1998). The increased mortality after PA is due to the asphyxia itself. Asphyxiated pups receive similar maternal care than normal vaginally born animals (Levine and Boksa 2006). Female pups are less sensitive to the damaging effects of PA and have higher survival rates (Loidl, Gavilanes et al. 2000, El-Khodor and Boksa 2003, Laplante, Brake et al. 2012). Similar to the Apgar scores in human infants, the severity of asphyxia can also be graded on behavioral features shortly after birth in experimental rats. Immediately after birth, C-section or vaginally delivered pups and pups with mild asphyxia start to breathe with a gluttonous gasp, which is rapidly replaced by regular breathing with intense vocalization and motility. When the duration of asphyxia exceeds 16 minutes, the pups are akinetic and do not vocalize; even at 80 minutes after delivery. Furthermore, these pups gasp with accompanying cyanosis, tremor, and sporadic clonic movements. Pups exposed to severe PA (for 19-21 min) require skillful resuscitation since they take a long time to start gasping and a longer time to establish regular breathing, largely supported by gasping (Chen, Engidawork et al. 1997, Dell'Anna, Chen et al. 1997, Marriott, Rojas-Mancilla et al. 2015). 


\subsection{The pathological effects of PA:}

\subsubsection{General changes in brain health, cellular stress and metabolism:}

In the original study, Bjelke, Andersson et al. (1991) found that male pups subjected to 16-17 min of PA weighed less than their control counterparts at 3 weeks of age. No differences in overall brain size or gross morphology were found. PA pups demonstrated a progressive and delayed increase in nuclear fragmentation (a marker of apoptosis) in comparison to control pups. These changes were most pronounced in the frontal cortex, the area surrounding the lateral ventricle, corpus callosum, dorsal striatum and cerebellum at 8 days after the insult and in the ventral-thalamus at 1-2 days after the insult. Apoptosis, rather than necrosis appears to be responsible for cell death in this model (Dell'Anna, Chen et al. 1997, Strackx, Zoer et al. 2010). Increased apoptosis was also evident in the grey and white-matter of the lumbar spinal cord, which may contribute further to motor pathologies found (de Louw, de Vente et al. 2002).

In human infants, HIE follows a three phase pattern of injury. The first phase is caused by a reduction in blood oxygen saturation, which reduces the oxygen supply to the brain. During hypoxic-ischemia, cerebral glucose use increases. In the immature brain, the capacity for glucose transport is less developed than the capacity for ketone body and lactate transport and there are thus lower glucose stores in the brain. The adult brain preferentially uses aerobic glucose metabolism to satisfy its energy needs, but the newborn brain can readily utilize other substrates like lactate and the ketone bodies $\beta$-hydroxybutyrate and acetoacetate. Accordingly, the energy production shifts to anaerobic glycolysis after HIE. The glycolysis pathway cannot produce sufficient ATP for the cerebral energy need and this initiates a state of cerebral energy failure, accompanied by lactate accumulation and acidosis, progressive neuronal depolarization and the extracellular accumulation of excitatory amino acids. The second phase of injury occurs upon re-oxygenation due to oxidative stress and the inactivation of buffering enzymes. This phase lasts from 6-72 hours after birth (reviewed by (Vannucci and Hagberg 2004, Perlman 2006, Marriott, Rojas-Mancilla et al. 2015)). The majority of neurons are lost in the second phase of injury. The third phase of neuron loss occurs during the repair phase, in the days and months after the injury. Persistent inflammation, epigenetic changes and an upregulation of physiological apoptosis can impair the survival and production of new neurons. This leads to a chronic state of neuronal loss (reviewed by (Davidson, Wassink et al. 2015)).

Several elements of this cascade have been validated experimentally in the 
model discussed. Mild PA (5-10 minutes) caused an increase systemic partial oxygen pressure $\left(\mathrm{pO}_{2}\right)$ immediately after the insult, which normalized within an hour and did not alter the systemic $\mathrm{pCO}_{2}$ (Berger, Vaillancourt et al. 2000). Longer periods of PA (5-20 minutes) caused a decrease in brain $\mathrm{pH}$ and an increase in glucose and cerebral blood flow within 10 minutes after the insult. The severity varied according to the length of the insult. Glucose transporters and the utilization of glucose by hexokinase and phosphofructokinase were not affected by PA in the early phase of injury (Lubec, Chiappe-Gutierrez et al. 2000). The switch to ketones was noted after 15 minutes of PA, through a direct reduction in the fatty acid synthase activity and increased availability the free fatty acids shortly after birth (Binienda, Holson et al. 1996).

Glycolysis and the corresponding increase in lactate levels occurred within 30 seconds after mild PA (for 5-10 minutes). The glycolysis is further exacerbated by administering anesthesia to the mother rat (Berger, Vaillancourt et al. 2000). At eighty minutes after PA, a widespread increase in brain lactate and acidosis was found, particularly in the basal ganglia structures. The degree of glycolysis in the striatum, ventral tegmental area (VTA), nucleus accumbens (NAcc), and the substantia nigra (SN) was dependent on the extent of the asphyctic insult. Mild insults (5-11 minutes) were sufficient to increase pyruvate formation (and thus glycolysis) in the SN/VTA. Whilst global brain acidosis was already evident after short durations of PA (for 5 minutes), only sustained insults exceeding 15-16 minutes decreased the cerebral $\mathrm{pH}$ below $\mathrm{pH}$ 7 and caused marked glycolysis (Chen, Engidawork et al. 1997, Engidawork, Chen et al. 1997).

The increases in brain lactate were preceded by peripheral markers of glycolysis and excitotoxicity including increased subcutaneous lactate, pyruvate, glutamate and aspartate levels (Dell'Anna, Chen et al. 1995, Chen, Engidawork et al. 1997, Engidawork, Chen et al. 1997). Increased peripheral and brain lactate levels were not accompanied by corresponding increased lactate metabolism (the oxidation of lactate, glucose and glycine was not changed) at 60 minutes after moderate PA (for 15 min) (Frizzo, Cardoso et al. 2010). After eighty minutes after mild-severe PA (15-22 min), there was a compensatory reduction of the excitatory neurotransmitter aspartate in the striatum(Chen, Engidawork et al. 1997).

With increased durations of PA, the energy metabolites adenosine triphosphate (ATP) and phosphocreatine was depleted in metabolically privileged organs - like the brain, heart and kidney (Seidl, Stöckler-Ipsiroglu et al. 2000). 
In the cerebral cortex, depleted ATP levels recovered within 60 minutes after PA (for $15 \mathrm{~min}$ ) (Souza, Martins et al. 2013). In energy metabolism, PolyADP polymerase-1 (PARP-1) competes with nicotinamide adenine dinucleotide $(\mathrm{NAD}+)$ for energy. Thus increased PARP-1 worsens energy availability in the brain. Immediately after severe PA (for $21 \mathrm{~min}$ ), PARP-1 activity was increased in the mesencephalon and it peaked within 1-8 hours. This activated downstream pro-inflammatory signaling cascades during the secondary damage phase. Eight hours after PA, the NF- $\kappa$ B pathway was activated and this resulted in translocation of $\mathrm{NF}-\kappa \mathrm{B}$ subunit $\mathrm{p} 65$ to the nucleus. This increased apoptosis at 24 hours after PA and the levels of the pro-inflammatory cytokines interleukin-1 $\beta$ (IL-1 $\beta$ ) and tumor necrosis factor- $\alpha$ (TNF- $\alpha$ ) (Allende-Castro, Espina-Marchant et al. 2012, Neira-Peña, Espina-Marchant et al. 2014, NeiraPeña, Rojas-Mancilla et al. 2015). In addition to the inflammatory cytokines, a large amount of reactive oxygen species (ROS) was released in the striatum and the cortex within 5 minutes after severe PA (for 19-20 min) (Capani, Loidl et al. 2001, Capani, Loidl et al. 2003). The increase in inflammatory cytokines at 24 hours was preceded by a global decrease. Within the first 2 hours after PA (15-20 min), the asphyxiated group had lower levels of IL-1 $\beta$, IL-6 and TNF- $\alpha$ in the whole brain and in the cerebellum. PA also increased IL-10 and decreased the ratio of phosphorylated signal transducer and activator of transcription 3 (pSTAT3) to un-phosphorylated STAT3 (Ashdown, Joita et al. 2008, Vlassaks, Strackx et al. 2013, Vlassaks, Brudek et al. 2014). Alterations in inflammatory cytokines were also seen in the placenta. Directly after severe PA (for $20 \mathrm{~min}$ ), the placenta of asphyxiated rats had lower levels of IL- $1 \beta$ and IL-10 than the placentas of C-section controls (Sparnaaij, Chedraui et al. 2016).

Not all pro-inflammatory cytokines are increased by PA and inflammation has been detected in a region specific manner. Severe PA (for $20 \mathrm{~min}$ ) did not alter the inflammatory cytokine enzyme prostaglandin E2. However, cyclooxygenase 2 (COX-2) transcription in the hippocampus was increased at 1-2 days after the insult and normalized at day 11. These changes in COX-2 transcription were not found in the cortex. Brain F2-isoprostane - a marker of oxidative injury - was increased within 2 hours after severe PA (for $20 \mathrm{~min}$ ) and returned to normal within 24 hours after the insult (Calamandrei, Venerosi et al. 2004, Piscopo, Bernardo et al. 2008). At the end of the early-phase of hypoxic damage at 6 hours, severe PA (for $20 \mathrm{~min}$ ) increased the transcription of many cellular stress response and cell signaling pathway genes. PA also altered transcripts that were active in the cell nucleus (Cox-Limpens, Vles et al. 2014). At this time-point the inflammatory cytokines, IL-6 and IL-10 were markedly increased, and IL- $1 \beta$ and TNF- $\alpha$ was moderately increased in the whole brain 
and cerebellum of the severe PA (for 20 min) group (Vlassaks, Strackx et al. 2013, Vlassaks, Brudek et al. 2014). In the secondary phase of hypoxic injury, at 12 hours after severe PA (for $20 \mathrm{~min}$ ), there was a marked increase in IL-6 and a reduction of the ratio of pSTAT3/STAT3 and TNF- $\alpha$ levels. At 24 hours after PA, TNF- $\alpha$ and the ratio of pSTAT3/STAT3 was moderately increased. IL-1 $\beta$ was slightly decreased. Across the whole brain, IL-10 was low in both asphyxia and control groups at 12-24 hours (Vlassaks, Strackx et al. 2013, Vlassaks, Brudek et al. 2014). In the first hours after birth, moderate PA (for $15 \mathrm{~min}$ ) also caused a transient increase in plasma adrenocorticotrophic hormone and testosterone levels. The testosterone increase was not mediated by increased luteinizing hormone and there did not appear to be long-term testosterone abnormalities after PA (Boksa and Zhang 2008).

Four days after severe PA (for $20 \mathrm{~min}$ ), in the recovery phase, the transcription of genes with a function in the cell nucleus was increased and the transcription of genes involved in cellular signaling and cellular stress was decreased. These transcriptional changes were reversed in comparison to changes seen during the acute damage phase at 6 hours (Cox-Limpens, Vles et al. 2014). A week after severe PA (for $20 \mathrm{~min}$ ), the inflammatory cytokines TNF- $\alpha$ and IL-10 were significantly decreased, but IL- $1 \beta$ remained increased in the cerebellum (Vlassaks, Strackx et al. 2013, Vlassaks, Brudek et al. 2014). Eight days after PA (5-20 min), the activity of RNA polymerase I - a component of the ribosomal RNA synthesis - and the levels of polymerase subunit PAF53 were reduced and the nucleolar structures were disintegrated. This indicated that PA continued to disrupt RNA and ultimately protein synthesis during recovery from the injury (Kastner, Mosgoeller et al. 2003). In humans, white matter is most vulnerable for injury during 23 and 32 weeks of gestation. This corresponds to P1-3 in the rat (Semple, Blomgren et al. 2013). In this rodent model, the injury occurs just before the vulnerable period of the white matter. Accordingly, reduced expression of myelin basic protein, a marker for mature oligodendrocytes, has been noted 19 days after severe PA (for $20 \mathrm{~min}$ ) (Piscopo, Bernardo et al. 2008). 


\subsection{The functional effects of PA:}

\subsubsection{Motor deficits:}

The delayed development of reflexes is an early indicator of neurological damage. At developmental stages where the rat brain development resembles the brain of a preterm (P1-3) and term human infant (P7-10) (Semple, Blomgren et al. 2013) the development of several reflexes are delayed by PA. In pups exposed to moderate PA (for $15 \mathrm{~min}$ ), the development of grasping and air-righting reflexes was delayed by 3-4 days. The development of negative geotaxis, sensory eyelid, gait, auditory startle reflexes occurred 1-2 days later in PA animals. These rats also had much slower reflexes, like the surface righting reflex, than their control littermates at 4-6 days. At 12-16 days, the PA pups had slower negative geotaxis and from 8-12 days they had a slower gait. These differences disappeared with age (Kiss, Szogyi et al. 2009, Kiss, Vadasz et al. 2013). At 7 and 12 days after PA (for $20 \mathrm{~min}$ ), PA animals demonstrated differences in spontaneous motor behavior. At 7 days, both PA females and males were more active at the start of the observation period than non-asphyxiated pups, while at 12 days the female PA pups were still hyperactive, while the male PA pups had become less active (Calamandrei, Venerosi et al. 2004).

At 3 weeks after the insult, when the rodent brain development resembles the brain of a 2-3 year old toddler (Semple, Blomgren et al. 2013), PA (for $15 \mathrm{~min}$ ) enhanced the apomorphine-induced locomotor response, whilst reducing rearing responses. There appeared to be an imbalance in dopaminergic movement control (Bjelke, Andersson et al. 1991). At the same time-point, PA (for $20 \mathrm{~min}$ ) reduced the distance moved in the open field test (Van De Berg, Kwaijtaal et al. 2003). After 4 weeks, corresponding to brain development of 4-11 years in the human (Semple, Blomgren et al. 2013), mild PA (for 15-16 $\mathrm{min}$ ) continued to increase amphetamine-induced locomotor responses. However, pups exposed to severe PA (for 19-20 min) had reduced locomotion and rearing (Chen, Bjelke et al. 1995). From week 3- 5, PA (for 15 min) pups had fewer footsteps on the grid-walking test and made more mistakes while walking on a grid. The errors were predominantly made with the forelimbs. The PA rats took longer to leave the inner and outer circled on the walk initiation test. Other movement tests, including the rope suspension, open board and rotarod tests were not significantly altered by PA (Kiss, Szogyi et al. 2009, Kiss, Vadasz et al. 2013). The observation that mild PA increased and severe PA decreased the locomotor response is reflective of the human clinical scenario. Infants with severe PA encephalopathy are at risk to develop cerebral palsy, while the long-term behavioral consequences in infants exposed to mild 
asphyxia are harder to determine. In this subgroup, an increased incidence of hyperactivity has been reported (Van Handel, Swaab et al. 2007).

Weeks 5-7 of rodent neurodevelopment correspond to years 12-18 of human brain development (Semple, Blomgren et al. 2013). At 6 weeks, severe PA (for $20 \mathrm{~min}$ ) did not influence gripping but it increased the stride width during normal walking patterns (Van De Berg, Kwaijtaal et al. 2003). PA (for 15 min) did also not change methamphetamine-induced locomotion (Wakuda, Matsuzaki et al. 2008). When comparing the effects of PA (for $10 \mathrm{~min}$ ) on male pups pre-puberty (at 5 weeks) and post-puberty (at 2 months), the pre-pubertal PA pups had similar levels of locomotor activity than controls. However, after puberty in young adulthood, PA rats had more spontaneous locomotor activity. Administration of apomorphine or the restriction stress test increased the activity of PA pups at both ages. Administration of amphetamine only increased locomotion 2 months after PA. These behaviors were linked to the mesolimbic dopaminergic system and shows that puberty exacerbated the dopamine-related behavioral changes in PA animals (Klawitter, Morales et al. 2007).

Birth complications are thought to contribute to the development of schizophrenia (Matheson, Shepherd et al. 2011). This disease typically presents in late adolescence and early adulthood, with a higher incidence in males. In addition to abnormalities in dopaminergic signaling, these patients also have deficits in sensorimotor gating and an increased sensitivity to stimulant drugs like amphetamine (Brisch, Saniotis et al. 2014). At 2 months of age (when neurodevelopment resembles a 20 year old human), mild PA (for 15 minutes) caused sensorimotor gating deficits in male pups. This was observed as a decreased pre-pulse inhibition of the startle response. Female PA pups had an increased pre-pulse inhibition. Male pups were also more sensitive for apomorphine induced-reduction of pre-pulse inhibition - modeling an increased sensitivity to dopaminergic signaling (Laplante, Brake et al. 2012). Dopaminergic function was approximately halved in the prefrontal cortex and increased by $40 \%$ in the NAcc and striatum of pups delivered by C-section with or without 15 minutes of PA (El-Khodor and Boksa 1997).

At 2.5 months, PA (for 10, 15 or 20 min) did not alter basal or amphetamineinduced locomotor activity (Venerosi, Valanzano et al. 2004, Laplante, Brake et al. 2012). However, at 3 months the PA group had elevated methamphetamineinduced locomotion accompanied by increased dopamine release in the NAcc (Wakuda, Matsuzaki et al. 2008). At 3 months, dopaminergic function and 
stress-induced dopamine release was increased in the NAcc and blunted in the right medial prefrontal cortex of PA animals. Dopamine transporter density in the prefrontal cortex was increased by mild PA (for $15 \mathrm{~min}$ ). This reduced inhibitory signals from the medial prefrontal cortex to the NAcc and lead to more spontaneous locomotor behavior at 3 months of age. These effects were also seen in C-section pups without asphyxia, but appeared to be aggravated by the asphyxia (Brake, Noel et al. 1997, Brake, Sullivan et al. 2000). C-section with/without PA (for $15 \mathrm{~min}$ ) increased the locomotor response to a single dose of amphetamine, whilst the PA group also became sensitized to repeated administration of amphetamine (Brake, Boksa et al. 1997, El-Khodor and Boksa 1998). If the mother was anesthetized with isoflurane $/ \mathrm{N}_{2} \mathrm{O}$ prior to the PA procedure, greater amphetamine sensitization was noted in the pups (Vaillancourt and Boksa 1998). Repeated cocaine administration increased locomotor activity in control and PA (for 15 min) pups, but not in PA (for 20 min) pups. In comparison to control vaginal delivery and C-section only pups, PA (for $15 \mathrm{~min}$ ) increased the sensitization response to cocaine, whilst PA (for 20 min) decreased sensitization. These results show that the length of PA influenced dopaminergic-mediated response in mature rats (Galeano, Romero et al. 2013). Severe PA (for 20 minutes) impaired motor coordination on the rotarod test at 3 months. However no gross changes in motor activity in the Y-maze and Barnes maze were found in the PA groups (Simola, Bustamante et al. 2008).

At 4 months, male pups that were subjected to PA (for 10 minutes) showed a hyperactive locomotor response to repeated stress, linked to increased mesolimbic dopamine release. The stress consisted of a daily tail pinch for 8 days and another pinch during a stress test 14 days later (El-Khodor and Boksa 2000). At 5 months of age, male PA (15 or $20 \mathrm{~min}$ ) pups had lower locomotor activity. Female pups did not show similar movement impairment (Loidl, Gavilanes et al. 2000). Interestingly, 10-15 minutes of PA reduced the preference for ethanol intake and the PA pups were also more sensitive to locomotor suppression by low doses of ethanol (Boksa 1998). All together, these observations indicate that mild-moderate PA could be useful as a model of the neurodevelopmental origin of schizophrenia and addiction disorders, whilst severe PA models induce progressive motor impairment in male rats.

6 months after severe PA (for $20 \mathrm{~min}$ ), the asphyxiated group covered a smaller distance in the open field test and elevated zero-maze than controls, although there were no differences in velocity between the groups. These differences could be due to affected locomotion, or due to increased anxiety in the PA animals (Strackx, Van den Hove et al. 2010). At 8 months, severe 
PA (for $20 \mathrm{~min}$ ) rats had less spontaneous locomotor activity during the first 10 minutes of a 70 minute open field test. After 10 minutes, the amount of activity was comparable between the PA and control rats. Administration of a dopamine D1 receptor agonist increased the locomotor activity (mainly crossing and wall rearing) in the PA group in the last 20 minutes of the test. Increased head moving was a stereotypical effect of D1 agonists. The PA group spent had a weaker stereotypic response to the drug. These findings indicate that PA may affect the function of D1 receptors in adult rats (Venerosi, Valanzano et al. 2004). 2 years after the insult, aged female PA (for $20 \mathrm{~min}$ ) rats did not have lower locomotion in the open field test (Weitzdoerfer, Gerstl et al. 2004). Thus we conclude that the effect of PA on locomotion depends on the length of the asphyxia and the sex of the animal.

\subsubsection{Social deficits and anxiety:}

The ultrasonic vocalization of rat pups is similar to crying in human infants (Zeskind, McMurray et al. 2011). These sounds alert the mother rats to a caregiving need and facilitate location of the pups. The amount of ultrasonic vocalizations is low shortly after birth and peaks at P11-P12. Up to P15, vocalizations in rat pups are primarily a reflexive response to variations in environmental temperature and isolation, while after this time-point the vocalizations change to a more social behavior in response to social cues (Zeskind, McMurray et al. 2011). At P11-13, male pups that had severe PA (for $20 \mathrm{~min}$ ) and pups that were born with C-section without asphyxia emitted more ultrasound vocalizations than vaginally born pups - which likely indicate increased anxiety and fear. PA and C-section per se may affect the maturation of the response of the pups to adapt to maternal separation. Female PA pups had less vocalization than male pups but they still had higher vocalization rates than the control pups (Calamandrei, Venerosi et al. 2004, Venerosi, Cutuli et al. 2006).

At 30-35 days, just before puberty, male rats with severe PA (for 19-20 min) had a markedly different response to stressful situations and antisocial behavior. During social interaction with an unfamiliar rat, rats with PA displayed less play-soliciting behavior and had increased defecation. These changes were not due to increased general anxiety measured in the open field test (Vázquez-Borsetti, Peña et al. 2016). However, the PA rats elicited more ultrasonic vocalizations after a fear-conditioning stimulus and thus had an increased anxiety in anticipation of an unavoidable shock (Venerosi, Cutuli et al. 2006). In another type of fear paradigm, the elevated plus maze test, 3 
month old pups that were exposed to moderate to severe PA (15 or $20 \mathrm{~min}$ ) showed reduced anxiety-related behavior (Hoeger, Engelmann et al. 2000). The innate fear elicited by the open space in the elevated maze test may differ both quantitatively and qualitatively from fear induced by inescapable shocks (Venerosi, Cutuli et al. 2006). At the same age, mild or severe PA (10 or $20 \mathrm{~min}$ ) had no influence on the novelty preferences of the rats (Venerosi, Valanzano et al. 2004).

At 2-3 months, severe PA (for 20-21 min) pups were more anxious, in both the elevated-plus maze and the open field test. In the open field, the PA pups spend more time close to the walls of the open field arena during the first 5 minutes of the test. These animals avoided exploring areas where they were not in contact with a solid body (Morales, Simola et al. 2010, Allende-Castro, Espina-Marchant et al. 2012). At 6 months of age, PA (for 10 or 20 min) rats were chronically stressed for 12 days by combining restraint stress on the first and last days, with a forced-cold water swim test. The PA (for 20 min) group had a slower rise in plasma corticosterone levels (Venerosi, Valanzano et al. 2004). PA (for $20 \mathrm{~min}$ ) rats had no differences in corticosterone response to a single stressor at 6 months (Strackx, Van den Hove et al. 2010). There were also no differences in plasma corticosterone after PA (for $20 \mathrm{~min}$ ) during the first month after the insult (Scheepens, Wassink et al. 2003). At 17 months, rats exposed to severe PA (for $20 \mathrm{~min}$ ) did not have increased anxiety in the elevated plus maze or the open field test (Galeano, Blanco et al. 2014). Two years after PA (for $20 \mathrm{~min}$ ), female rats spent more time grooming themselves and less time fighting. These rats also spent less time sniffing their partner, and more time running alone - indicating lower levels of aggression, but also less social exploration. The PA group also had significantly more anxietyrelated behavior and decreased autonomous functions (Weitzdoerfer, Gerstl et al. 2004).

In addition to anxiety and reduced social behavior, PA also reduced the motherly instincts of surviving pups. Female PA (for $20 \mathrm{~min}$ ) rats that grew up and become mothers themselves, left the nesting area and self-groomed for longer periods than control C-section mothers during the first 2 weeks after the birth of their pups. They also licked the pups less. After separation from their pups on day 4 of lactation, the PA mothers did not retrieve all of their pups and they took longer to retrieve them. In trials of fear behavior, the PA mothers froze quicker after a fearful stimulus and they were thus less able to filter out irrelevant stimuli whilst caring for their offspring. These behaviors may be due to a deficit in the dopaminergic arousal stimuli (Cirulli, Bonsignore et al. 
2003, Bonsignore, Venerosi et al. 2006). Collectively, these studies show that increased anxiety is a common occurrence across several PA studies throughout the lifetime of the rat. This correlates with observations that children with mild PA have a higher incidence of emotional and behavioral problems (Van Handel, Swaab et al. 2007) and that birth complications are a risk factor for adult-onset mental illness(Matheson, Shepherd et al. 2011).

\subsubsection{Cognition:}

Cognition and learning is a third functional domain adversely impacted by PA. Impairment ranges from relatively rare mental retardation in severe cases, to collectively lower average academic scores within the normal range in children with mild encephalopathy (Van Handel, Swaab et al. 2007, Volpe 2012).

Reports of cognitive impairment in PA rodents vary according to the domain of functioning assessed and age. Cognitive impairment is not consistently detected in this model. At 30 days after severe PA (for $20 \mathrm{~min}$ ), rats had an enhancement in habituation to repeated locomotion measurements in the open field; normal associative memory to an aversive stimulus (Saraceno, Caceres et al. 2016); mild deficits in recognition memory (assessed with the novel object recognition test) and mild deficits in spatial learning and reference memory (assessed with the Morris Water Maze) (Blanco, Galeano et al. 2015). This was not replicated by a study at 6 weeks after moderate-severe PA (15 or 20 minutes), in which no deficits in the Morris Water Maze were detected (Boksa, Krishnamurthy et al. 1995, Loidl, Gavilanes et al. 2000) or a test of spatial object location memory at 10 weeks after mild and moderate PA (10 or 20 min) (Venerosi, Valanzano et al. 2004).

At 3 months, during young adulthood, severe PA (for 20-21 min) rats had deficits in the non-spatial working memory - as assessed by the novel object recognition test. However, deficits in spatial memory - as assessed by Y maze and Barnes maze - were not apparent (Simola, Bustamante et al. 2008, Morales, Simola et al. 2010, Flores-Balter, Cordova-Jadue et al. 2016). At 4 months, spatial working memory deficits were seen in PA $(10,15$ or $20 \mathrm{~min}$ ) rats through impaired initial acquisition in the Morris Water Maze and slight long-term memory retention deficits (Boksa, Krishnamurthy et al. 1995). Six months after PA severe (for $20 \mathrm{~min}$ ), the asphyxiated group had worse recognition memory (as scored by the novel object recognition test) at both short (1 hour) and long (24 hour) testing intervals (Strackx, Van den 
Hove et al. 2010). At 17- 18 months, PA (19-20) exaggerated age-related long-term spatial working memory and spatial reference memory impairment in the Morris Water Maze. Short-term spatial memory and object recognition was not affected by PA (Van de Berg, Blokland et al. 2000, Galeano, Blanco et al. 2014). Table 5.1 gives an overview of the effect of PA on behavior.

\subsection{The molecular effects of PA on neurotransmitter systems:}

\subsubsection{Dopaminergic transmission and regulation in the basal ganglia:}

Alterations in dopaminergic neurotransmission were one of the first changes reported in this model, and these changes have been consistently reported across several studies. Shortly (20-40 minutes) after mild-severe PA (for 15-20 min) dopamine levels were increased in the SN/VTA and the NAcc/olfactory tubercle. Longer periods of asphyxia produced a greater increase in dopamine in these regions. This was accompanied by an increase in the dopamine metabolite DOPAC in these regions and increased homovanillic acid in the SN/VTA, NAcc/olfactory tubercle and the striatum without altered reactivity of tyrosine hydroxylase $(\mathrm{TH})$, the rate-limiting enzyme in the dopamine synthesis pathway. These results indicate increased dopamine release, but not increased synthesis right after PA (Ungethüm, Chen et al. 1996).

Within 80 minutes of birth (C-section) dopamine distributes across the brain in accordance with the regional segregation of nerve terminals and cell bodies of the mesotelencephalic dopaminergic systems. Hence, control rats had higher dopamine and dopamine-metabolite levels in the striatum and NAcc than in the SN or the VTA. The changes in dopamine distribution due to PA depend on the length of the asphyctic insult. 19-21 minutes of PA globally increased the levels extracellular dopamine by reducing dopamine catabolism. In the SN, 10-20 minutes of PA caused a 3-fold increase in dopamine levels, but 21-22 minutes of PA caused a $50 \%$ reduction. In the VTA, dopamine levels were increased 5-fold by 5-11 minutes of PA (Chen, Engidawork et al. 1997). At 24 hours after birth (vaginal delivery), the expression of TH (and thus dopamine synthesis) peaks in the SN/VTA, striatum and limbic areas and then stabilizes. Mild-severe PA (15-20 min) increased the overall SN/VTA TH expression during the recovery phase (6 hours to 1 week after birth), but it did not increase the 24 hour peak. In the striatum and limbic areas, PA only increased TH expression at 1 week after birth. The dopamine D2 receptor can 
Table 5.1: An overview of behavioural changes seen in male rats relative to controls ( $\uparrow=$ increase, $\downarrow=$ decrease, $0=$ no changes)

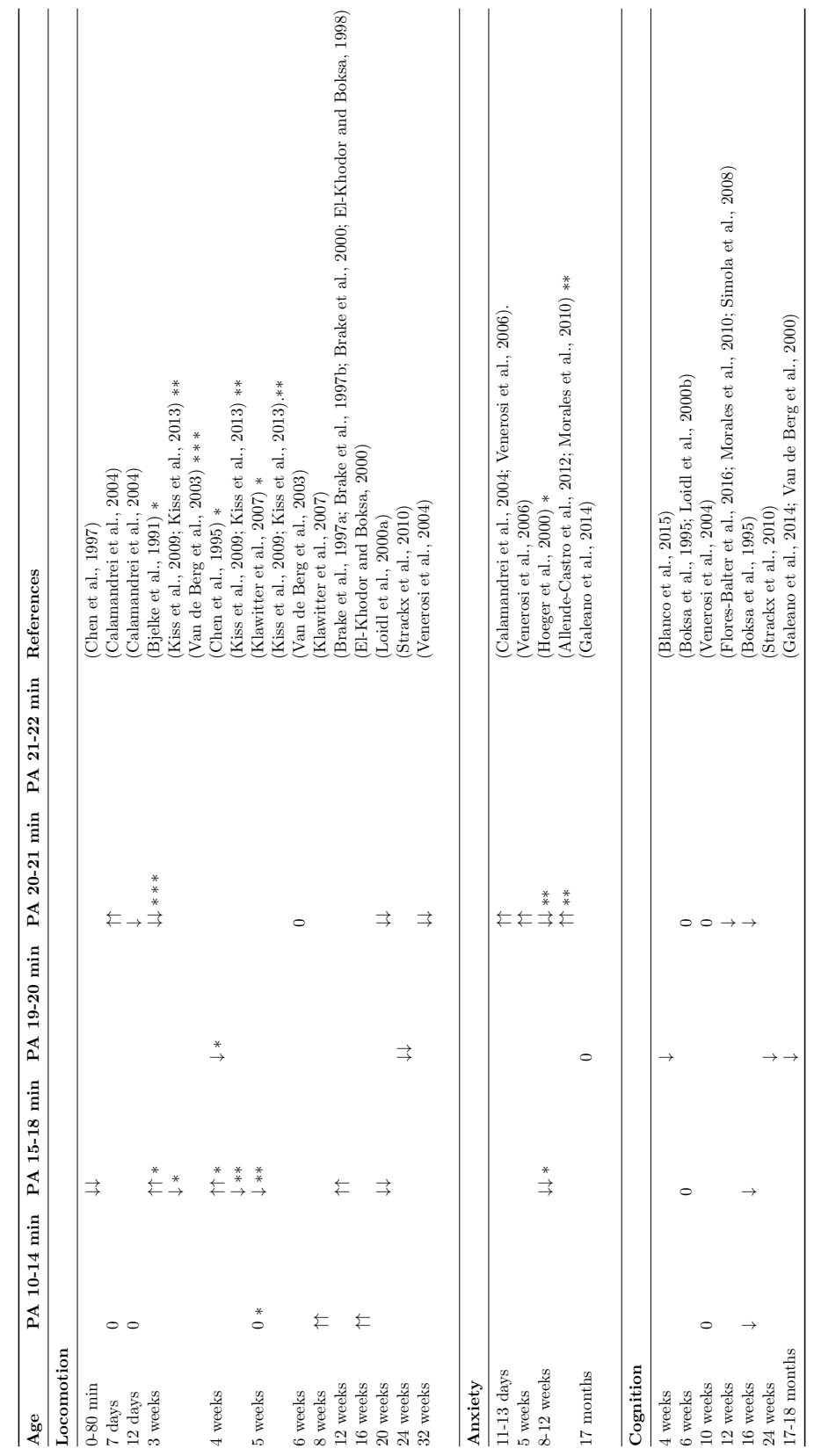


act as an auto-receptor and prevent the further release of dopamine through negative feedback. In the SN/VTA, the levels of the D2 receptor- but not the D1 receptor - increased in parallel with TH expression as a normalization response to increased dopamine release after PA. Increased expression of the D2 receptor may also protect the mesencephalic dopaminergic neurons from glutamate- and oxidative stress-induced cytotoxicity (Gross, Müller et al. 2000, Gross, Andersson et al. 2005). Eight days after PA, dopamine levels in the SN were either increased more than 2-fold in pups exposed to 20-21 minutes of PA or reduced in pups exposed to 21-22 minutes of PA. The dopamine metabolite, DOPAC, was also decreased in pups exposed to 21-22 minutes of PA - thus sub-lethal PA reduced dopamine through reduced synthesis, not increased catabolism. In the striatum, the effect of 2-22 minutes of PA on dopamine was less clear, but also there the levels of the dopamine metabolites DOPAC and homovanillic acid (only sub-lethal PA (21-22 min)) were reduced - thus dopamine catabolism was also reduced in the striatum, while glycolysis in this region was increased (Chen, Engidawork et al. 1997).

At weaning - three weeks after moderate PA (for $15 \mathrm{~min}$ ) - there were more TH-immunoreactive neurons in the SN pars compacta, SN zona lateralis and VTA. No changes in TH-immunoreactivity were found in the caudate nucleus and NAcc of the striatum (Bjelke, Andersson et al. 1991). At the same age, severe PA (for $20 \mathrm{~min}$ ) reduced the total amount of striatal neurons by $16 \%$ in male rats. This neuron loss was preceded by exaggerated apoptosis in the first week after PA (Van de Berg, Schmitz et al. 2002).

Several studies were done on the dopaminergic system in 4 week old rats. Homogenates of brain regions of male pups showed that severe PA (for 19-20 min) reduced the total amount of dopamine in the SN/VTA, striatum and the NAcc/olfactory tubercle. These changes were not seen after moderate PA (for 15-16 minutes). The dopamine metabolites DOPAC, homovanillic acid and 3-methyl tyrosine levels were not altered by PA in these brain regions, indicating that the reduced dopamine levels were not due to increased dopamine metabolism. Measurements of the enzymatic activity of TH showed no difference due to PA (Ungethüm, Chen et al. 1996). However, the amount of TH-immunoreactive neurons in the SN/VTA was increased after severe PA (19-20) in male rats (but not after moderate PA (for 15-16 min)). This increase could be counteracted by administering the cholinergic agonist nicotine and suggests that a misbalance in dopaminergic and cholinergic signaling contributed to these changes (Chen, Bjelke et al. 1995). The reduction of dopamine levels in the NAcc was replicated by a study of mixed sex pups with PA (for 15 and 
19 min), but a paradoxical increase in dopamine in the SN/VTA was seen after severe PA (for $20 \mathrm{~min}$ ) and to a lesser extent after moderate PA (for $15 \mathrm{~min}$ ) (Bustamante, Goiny et al. 2003). In vivo microdialysis experiments, with mixed sex pups, show increased basal extracellular dopamine, DOPAC and homovanillic acid concentrations in the dorsal striatum, after severe PA (for 20-21 $\mathrm{min}$ ) but reduced basal concentrations of these metabolites once the duration of PA exceeded 21 minutes. Severe PA (20-22 min) also strongly reduced the release of further dopamine upon depolarizing conditions indicating a long-term impairment of a firing-dependent release from striatal dopaminergic terminals (Chen, Engidawork et al. 1997).

PA also had an effect on the dopamine receptors. PA (15-20 min) increased the amount D1 and D2 receptors in the dorsal striatum of mixed sex pups (Gross, Müller et al. 2000) and reduced the agonist affinity of the D2 receptor in this region. PA (15-20 min) also reduced the amount of D1 receptors in the NAcc, olfactory tubercle and SN and caused a compensatory increase in the affinity of the D1 receptor for its agonist in the first two regions. In the NAcc severe PA (for 19-20 min) also reduced D2 receptor agonist affinity. Moderate PA (for 15-16 min) increased D3 receptor agonist binding in the dorsal striatum (Chen, Hillefors-Berglund et al. 1997). Together these studies indicate lasting abnormalities in dopaminergic signaling after PA. These abnormalities were more pronounced after increasing durations of PA and in studies that used only male offspring.

In 2 month old rats, C-section birth by itself increased dopamine in the NAcc and dorsal striatum of male - but not female - pups. In pups with additional moderate PA (for $15 \mathrm{~min}$ ), the dopamine levels were similar to vaginally born controls for both sexes (El-Khodor and Boksa 2003). In 3 month old rats, PA (16 or $20 \mathrm{~min}$ ) reduced the amphetamine-induced extracellular dopamine concentrations in the lateral striatum by approximately $30 \%$ and $70 \%$, respectively. PA also reduced the dopamine release in response to $\mathrm{K}^{+}$ depolarization in the same groups by $45 \%$ and $83 \%$ respectively (Bustamante, Morales et al. 2007). At the same age, repeated cocaine administration reduced TH expression in the NAcc of both rats that were exposed to PA and control animals. Moderate PA (for $15 \mathrm{~min}$ ) rats had a greater reduction in $\mathrm{TH}$ than controls, whilst the cocaine-induced changes seen after severe PA (for 20 min) were smaller than in controls. Neither cocaine nor PA altered the expression of the dopamine active transporter (Galeano, Romero et al. 2013). Moderate PA (for $15 \mathrm{~min}$ ) increased dopamine and DOPAC in the prefrontal dopaminergic system and NAcc of male rats; decreased both products in female 
rats and increased dopamine in the amygdala of both sexes (Laplante, Brake et al. 2012). Mild-severe PA (10-20 minutes) reduced TH-immunoreactive neurons in the globus pallidus. Severe PA (for $20 \mathrm{~min}$ ) reduced the expression of the vesicular acetylcholine transporter (VAChT) and increased vesicular monoamine transporter (VMAT) reactive fibers in the striatum, contributing to the misbalance between cholinergic and monoaminergic neurotransmission (Kohlhauser, Mosgoeller et al. 1999).

At 4.5 months after PA, C-section, but not C-section and moderate PA (for $15 \mathrm{~min}$ ), reduced the D1 receptor binding in the infralimbic cortex, anterior olfactory nucleus, NAcc, core tubercles and the olfactory tubercles; and increased NMDA receptor binding in the cingulate cortex. Repeated tail-pinch stress, for 2 weeks before the analysis, further decreased D1 receptor binding in the infralimbic cortex and the anterior olfactory nucleus and increased D4 receptor binding in the dorsal striatum, NAcc and olfactory tubercles of the C-section only group. C-section, but not C-section plus PA, reduced D3 receptor binding in the NAcc in response to stress. Stress reduced D1 receptor binding in the lateral frontal and cingulate cortex; and increased D2 receptor-family binding in the NAcc, regardless of the birth group. In many regions, the changes seen after 15 minutes PA were similar to the vaginal birth control group. Only Csection by itself significantly altered the pattern of dopamine receptor binding (El-Khodor and Boksa 2001, El-Khodor, Flores et al. 2004). Six months after severe PA (for $20 \mathrm{~min}$ ) the basal and amphetamine-induced dopamine release; and dopamine turnover was decreased in the striatum. The decrease in the SN was less pronounced (Loidl, Herrera-Marschitz et al. 1994).

In conclusion, sustained duration-dependent abnormalities in dopaminergic signaling have been consistently reported in this model across several studies. The structures within the basal ganglia are especially well-studied in this model and vulnerable to the insult. These observations support the consistent locomotor and anxiety-related behavior problems seen in this model and in human infants with PA. Several studies have shown that not only basal dopamine levels are altered, but also dopamine release in response to stress and stimulants. This highlights the usefulness of this model to study the long-term psychiatric changes due to birth complications. 


\subsubsection{GABAergic and glutamatergic transmission in the basal ganglia:}

Glutamate and gamma-amino butyric acid (GABA) are respectively the major excitatory and inhibitory neurotransmitters in the brain. Both systems are interlinked with dopaminergic neurotransmission. Excitoxocity due to excessive glutamate release is central to the acute neurotoxic cascade after PA (Perlman 2006). A disruption in the balance between excitatory and inhibitory synaptogenesis is thought to underlie several neurodevelopmental disorders also linked to PA, including autism, epilepsy and schizophrenia. GABAergic interneurons control neuronal excitability, integration, and plasticity. These neurons also regulate the generation of temporal synchrony and oscillatory behavior among networks of pyramidal neurons. Such oscillations within and across neural systems are believed to serve various complex functions, such as perception, movement initiation, and memory (Gatto and Broadie 2010, Chattopadhyaya and Di Cristo 2012).

An hour after moderate PA (for $15 \mathrm{~min}$ ), glutamate uptake in the cerebral cortex (Souza, Martins et al. 2013) and GABA uptake in the hippocampus was unaltered. PA reduced glutamate uptake in the hippocampus and also transiently increased the levels of the $\mathrm{P}_{2} \mathrm{X}_{7}$ purine receptor (involved in glutamate release) in response to ATP signaling (Frizzo, Cardoso et al. 2010). In the SN and striatum, glutamate levels increased between 40-120 minutes after the insult after all durations of PA (2-21 min). Striatal GABA levels only significantly increased after severe PA (for 20-21 minutes). In the developing brain, GABA has excitatory properties, which are later replaced by its classic inhibitory actions. Together these results support the role of excitotoxicity as a major mechanism of PA (Chen, Engidawork et al. 1997).

During childhood, at 1 month after mild-severe PA (2-20 minutes), striatal extracellular glutamate and GABA levels remained elevated and GABA release upon a depolarizing $\mathrm{K}^{+}$stimulus was increased. At this age, GABA functions as an inhibitory neurotransmitter which can counteract the excitatory effects of glutamate. After sub-lethal PA (21-22 min) the striatal glutamate levels declined (Chen, Engidawork et al. 1997). At 5 weeks after severe PA (for $20 \mathrm{~min}$ ), the amount of GABAergic neurons expressing reelin in the medial prefrontal cortex were reduced in deep cortical layer VI and the amount of calbindin interneurons were reduced in the superficial layer II. These changes were not seen across all the cortical layers and it was not due to an overall neuron loss in the prefrontal cortex. Thinning of the medial prefrontal cortex and reduced reelin expression is associated with schizophrenia (Vázquez-Borsetti, 
Peña et al. 2016).

At 4.5 months after PA, in adulthood, C-section by itself or with additional PA (for $15 \mathrm{~min}$ ) increased the glutamatergic AMPA receptors in the NAcc and NMDA receptors in the anterior olfactory nucleus. C-section with/without PA also reduced basal kainate receptor binding and increased stress-induced kainate receptor binding in the olfactory tubercle. Stress decreased AMPA-receptor binding in NAcc of the PA group (El-Khodor, Flores et al. 2004). At 6 months after PA (for $20 \mathrm{~min}$ ), glutamate and aspartate levels were reduced in the striatum and GABA levels was reduced in the SN (Loidl, Herrera-Marschitz et al. 1994). The lasting changes in GABA-neurotransmission were also evident in the structure of GABAergic neurons.

Changes in the neuronal cytoskeleton had been implicated in many neurodegenerative diseases. The GABAergic neurons play an important role in suppressing excessive dopamine release. At 1-2 months after PA (for 19-20 min), the GABAergic medium spiny neurons and the GABAergic interneurons in the striatum were reduced by $22 \%$ and $43 \%$ respectively (Van De Berg, Kwaijtaal et al. 2003) and the thickness of the post-synaptic densities (PSD) of these neurons was increased. These increases occurred in pups that had more than 10 minutes of PA and peaked in pups exposed to 19 minutes of PA. Male pups were more susceptible than female pups and developed increased PSD thickness at shorter durations of PA. The increase in PSD thickness can partially be ascribed to increased amounts of $\mathrm{F}$-actin and $\beta$-actin in the dendritic spines. F-actin concentrates in the mushroom-type dendritic spines and PA likely increased the amount of these spines. In the PA (for $20 \mathrm{~min}$ ) animals, ubiquitination of the PSDs was increased. Ubiquitin primarily attaches to damaged proteins. The increased density of the PSD after PA may reflect an increase in damaged proteins (Cebral and Loidl 2011, Grimaldi, Romer et al. 2012, Saraceno, Ayala et al. 2012, Muñiz, Romero et al. 2014). Shorter durations of PA (for $10 \mathrm{~min}$ ) increased the dendrite density of the medium spiny neurons of the NAcc at 7 weeks, but not 10 weeks after the insult (Juárez, Gratton et al. 2008).

At 6 months after PA (for 19-20 min), increased astrogliosis and the accumulation of high molecular weight neurofilaments in the axon bundles in the striatum was observed (Cebral, Capani et al. 2006). This caused a loss of GABA-ergic neurons with slight (after PA 10-15 min) to marked condensation (after PA 19-20 min) of the neuronal nuclei undergoing apoptosis. PA did not affect the integrity of pre-synaptic terminals, pre-synaptic vesicles and the ultrastructure of the post-synaptic densities (PSDs). However, PA increased 
the length and thickness of the PSDs. The PSDs also had irregular borders and fragmentation and higher levels of ubiquitination after PA (for $20 \mathrm{~min}$ ). The majority of synapses in the striatum originate from cortical-neostriatal glutamatergic afferent connections and these neurons were likely affected by PA (Capani, Saraceno et al. 2009). At 22 months of age, the striatal volume of rats that were exposed to severe PA (for $20 \mathrm{~min}$ ) was increased. As a result, they had more pre-synaptic boutons. The density of pre-synaptic boutons in this area was unchanged (Van de Berg, Blokland et al. 2000). An overview of the effects of PA on dopaminergic and GABAergic transmission is given in table 5.2.

\subsubsection{Serotonergic neurotransmission and tryptophan metabolism:}

The serotonergic (5-hydroxytriptamine) neurotransmitter system plays an important role in mood disorders and also in autism. Elevated plasma serotonin concentrations are seen in approximately a third of autism cases. Serotonin can modulate a number of developmental events, including cell division, neuronal migration, cell differentiation and synaptogenesis. Hyperserotonemia during fetal development results in the loss of serotonin terminals through negative feedback (Yang, Tan et al. 2014). 1 month after severe PA (for 20 min), levels of the serotonin metabolite 5-hydroxyindoleacetic acid (5-HIAA) - and thus presumably levels of serotonin - were increased in the SN and decreased in the striatum and the NAcc (Bustamante, Goiny et al. 2003). At 2-4 months after mild-severe PA (15-21 min), serotonin was not affected in most brain regions (El-Khodor and Boksa 2003, Allende-Castro, Espina-Marchant et al. 2012, Laplante, Brake et al. 2012). Only the NAcc demonstrated an increase in serotonin after PA (Laplante, Brake et al. 2012). 5-HIAA was moderately increased in the dorsal hippocampus of PA male pups and decreased in the thalamus of female PA pups (El-Khodor and Boksa 2003).

The serotonin precursor tryptophan can also be metabolized to kynurenic acid (a potentially neuroprotective glutamatergic NMDA-receptor antagonist), 3-hydroxy -kynurenine (a free radical generator) and quinolinic acid (an excitotoxin) via the kynurenine-metabolism pathway. Low levels of all of these compounds are needed for optimal NMDA-receptor functioning. In the first 10 minutes after PA (for 5-20 min), kynurenic acid was increased in a timedependent manner and 3-hydroxykynurenine was decreased after mild PA (for $5 \mathrm{~min}$ ), normalized after moderate PA (for $10 \mathrm{~min}$ ) and increased after severe PA (for 20 min) (Baran, Kepplinger et al. 2001). Kynurenic acid continued to be elevated in the first 6 hours after PA (for $15 \mathrm{~min}$ ), whilst overall 3hydroxykynurenine was decreased in the first 24 hours after PA (for $15 \mathrm{~min}$ ). 
Table 5.2: An overview of changes in neurotransmission in the SN/VTA and striatum/NAcc ( $\uparrow=$ increase, $\downarrow=$ decrease, $0=$ no changes)

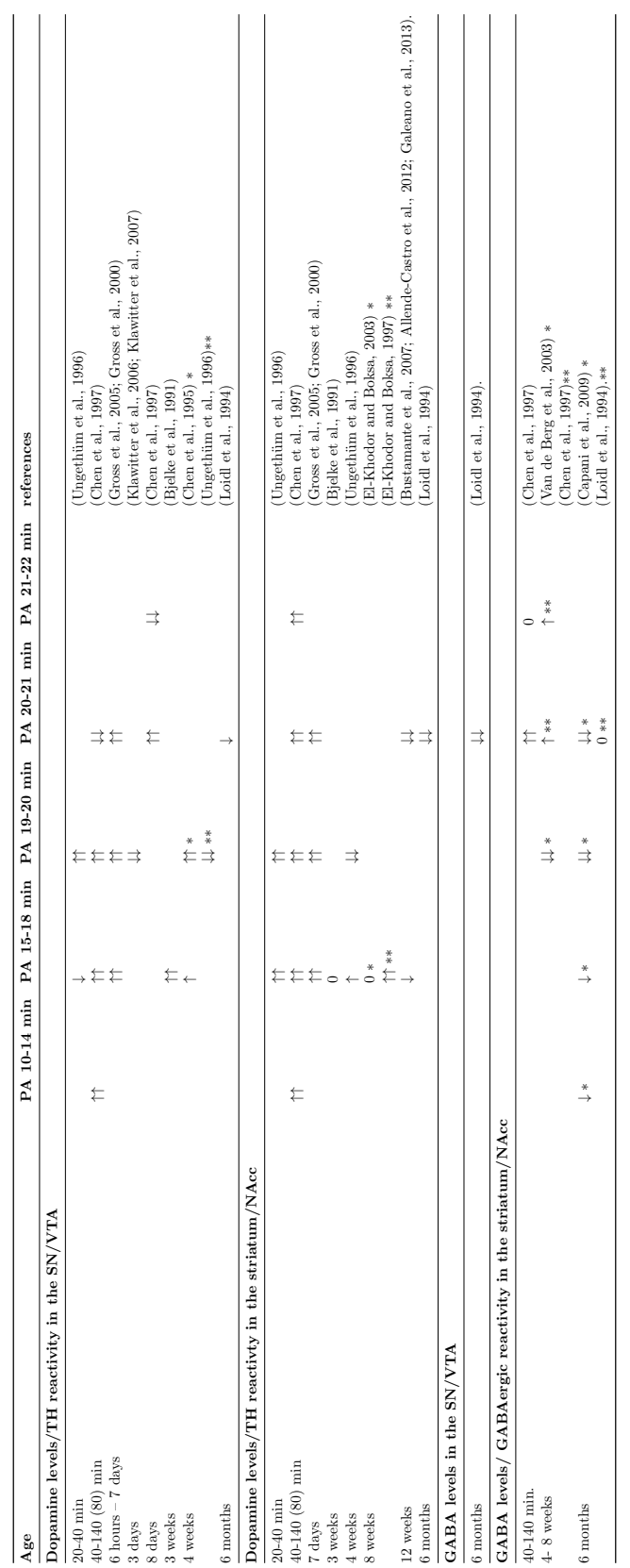


This was possible a protective compensatory reaction after PA (Ceresoli-Borroni and Schwarcz 2000, Ceresoli-Borroni and Schwarcz 2001). The administration of a kynurenine-3-hydroxylase inhibitor to the mother prior to PA (15 min), further increased kynurenic acid and reduced 3-hydroxykynurenine and quinolinic acid in the offspring. The serotonin-metabolism pathway has potential as a therapeutic target to reduce excitotoxicity-mediated damage after PA (Ceresoli-Borroni, Guidetti et al. 2007). More studies on the serotonergic system in this model is needed, both as a potential therapy and to improve the understanding of the social and emotional deficits after PA.

\subsubsection{Cholinergic transmission and the cognitive structures:}

Several networks are involved in memory and cognition. The hippocampus and prefrontal are key structures in these processes. In both humans and rats, the hippocampus develops perinatally. The majority of pyramidal cells in the hippocampus are generated prenatally, but only about $15 \%$ of granule cells are present in the rat dentate gyrus (DG) at birth. In rats, cortical neurogenesis is present from gestational day 9.5 up to postnatal day P15 (Semple, Blomgren et al. 2013). Thus both of these regions are still developing at the time of the insult and minor changes in their growth can have severe long-term consequences. Cognitive impairments - ranging from rare reports of intellectual disability to lower average academic performance within the range of normal are common long-term consequences of PA (Van Handel, Swaab et al. 2007). In Alzheimer's disease both of these regions undergo neurodegeneration due to the formation of amyloidogenic plaques and oxidative stress. The presenilin proteins (PSEN1 and PSEN2) are important in the splicing of the amyloid precursor protein to $\beta$-amyloid (a major toxic protein in Alzheimer's disease). Genetic mutations that increase function of these proteins cause young-onset Alzheimer disease. PSEN1 and PSEN2 are also integral membrane proteins that are regulated during embryonic development and neurodegenerative insults. 1 day after severe PA (for 20 min), the expression of PSEN1 and PSEN2 in the cortex increased and 4 days after the insult PSEN1 and PSEN2 were up-regulated in the hippocampus, like in Alzheimer's disease. However, during recovery at 11 days after the insult, a compensatory lowering of presenilin levels in both brain regions was seen in the PA group (Piscopo, Bernardo et al. 2008).

The developing brain has lower anti-oxidant levels than the adult brain and may thus be more susceptible to the damaging effects of reactive oxygen species. At one day after severe PA (for $20 \mathrm{~min}$ ), a compensatory increase of the anti-oxidant enzymes Copper/Zinc superoxide dismutase, Manganese su- 
peroxide dismutase and catalase was seen in the hippocampus. These increases were transient and not noted in the cerebral cortex. At 4 days after the insult, the PA group had depleted their expression of manganese superoxide dismutase and catalase to levels below the controls and Copper/Zinc superoxide dismutase expression was similar to the expression in control pups (Piscopo, Bernardo et al. 2008). Three days after severe PA (for $20 \mathrm{~min}$ ), the heat-shock protein 90 co-chaperone $C d c 37$ and proteins involved in the redox system, protein synthesis, synapse formation, protein processing and nerve growth factors were increased the hippocampus - indicating increased activation of repair pathways (Weitzdörfer, Höger et al. 2008).

During the brain growth spurt, at 7 days after severe PA (for $20 \mathrm{~min}$ ), neuronal apoptosis of the non-proliferating cells in layer CA3 and the supraand infrapyramidal bands of the dentate gyrus of the hippocampus was increased. This was accompanied by an increase in the pro-apoptotic protein BAD and the anti-apoptotic protein BCL-2. The ratio of BAD/BCL-2 and the phosphorylation of ERK-2 were also increased. Although both pro-apoptotic and anti-apoptotic proteins were increased by PA, the increase in anti-apoptotic proteins was not sufficient to prevent cell death. PA also increased cellular and neuronal proliferation in the supra- and infrapyramidal band of dentate gyrus and the hilus (Morales, Fiedler et al. 2008).

From 2 days - 3 weeks after mild PA (for 10 min), the asphyxia group had a lower density of dendritic spines in the prefrontal cortex. The arborisation of the dendritic spines was also reduced from $3-5$ weeks and thus the ability to form synaptic contacts was reduced at that age. These changes were not evident at 10 weeks (Juárez, Gratton et al. 2008). In the hippocampus, mild PA (for $10 \mathrm{~min}$ ) reduced the dendritic spine density of the pyramidal neurons in the CA1 layer from 2 days - 2 weeks after the insult. From 3-5 weeks after PA, the density of the dendritic spines was increased and the length of the dendrites was decreased in comparison to vaginally born controls (Juárez, Gratton et al. 2008). At the time of weaning at 3 weeks, proteins associated with endocytosis and proteasome assembly in the hippocampus was also reduced by severe PA (for $20 \mathrm{~min}$ ) (Weitzdörfer, Höger et al. 2008) and moderate PA (for $15 \mathrm{~min}$ ) reduced the amount of pyramidal cell bodies in the hippocampal CA1 and CA3 layers (Bjelke, Andersson et al. 1991). At this time-point, PA induced hippocampal disruption was evident in both layers.

4 weeks after severe PA (for $20 \mathrm{~min}$ ) increased astrogliosis in the hippocampal CA1, CA3 and DG layers were observed. The endocannabinoid (EC) 
system is a lipid transmitter network involved in several physiological processes including synaptic plasticity, neurogenesis, memory, and mood. At this age, the EC and related acylethanolamide pathways were also altered by PA. An enzyme involved in EC synthesis and an acylethanolamide-related receptor were decreased in layers CA1 and CA3 of the dorsal hippocampus and the EC degradation enzyme Fatty Acid Amine Hydrolyse was increased in the CA3 region. Together, these changes likely reduced overall EC signaling which adversely impact neurogenesis in the hippocampus (Blanco, Galeano et al. 2015). Severe PA (for $20 \mathrm{~min}$ ) increased the degeneration of pyramidal neurons in the CA1 layer and increased the dendritic spine density of these neurons in the stratum radiatum. The increase in spine density was associated with increased signaling of the PI3K/Akt/GSK3 pathway, an important pathway for the regulation of synaptogenesis (Saraceno, Caceres et al. 2016). From 4-6.5 weeks, severe PA (for $20 \mathrm{~min}$ ) also caused synaptic reorganization in the stratum oriens of the CA3 layer, manifesting as mossy fiber sprouting (Morales, Fiedler et al. 2008, Morales, Simola et al. 2010). Mossy fiber sprouting is a form of synaptic reorganization which is frequently seen in patients with temporal-lobe epilepsy, a common consequence of early-life brain damage (Buckmaster 2014). Apoptosis was detected in the CA1 and CA3 layers of both control and PA rats, but the CA3 layer had an additional increase in apoptosis due to PA. In the DG, PA increased cellular and neuronal proliferation the suprapyramidal band (Morales, Fiedler et al. 2008, Morales, Simola et al. 2010). At 6.5 weeks, the increased thickness of the hippocampal postsynaptic densities persistent after mild-severe PA (10-20 min). A sex-dependent effect was seen, with a greater impairment found in the post-synaptic densities of male rats (Cebral and Loidl 2011).

3 months after moderate PA (for $15 \mathrm{~min}$ ), the amount of dentate granule cells in the hippocampus was reduced by proximately $20 \%$. The volume and cell counts the CA1 and CA3 layers were not affected (Wakuda, Matsuzaki et al. 2008). Severe PA (for $20 \mathrm{~min}$ ) globally reduced proteins associated with antioxidant and immune response and increased a serine protease inhibitor in the hippocampus (Weitzdörfer, Höger et al. 2008). The hippocampus, prefrontal cortex and cerebellum also had less TH-immunoreactivity at this age in PA (10-20 $\mathrm{min}$ ) rats and thus less innervation from dopaminergic structures. No changes were found in the monoaminergic, cholinergic and glutamatergic neurotransmitter transporter systems in the hippocampus and cerebellum. However, in the prefrontal cortex, the amount of excitatory amino acid carrier 1 (EAAC1)positive perikarya cells and the mRNA levels of catechol-o-methyl-transferase (COMT, only in moderate PA(for 15 min)) were increased - indicating gluta- 
matergic and dopaminergic transmission alterations (Kohlhauser, Mosgoeller et al. 1999, Wakuda, Iwata et al. 2015).

4 months after PA (for $20 \mathrm{~min}$ ), marked astrogliosis was found in the stratum radium of the hippocampus CA1 layer. In the same area, the apical dendrites had focal swelling and a fragmented appearance. The microtubule-associated protein-2 reactivity and phosphorylation of high and medium molecular weight neurofilaments was reduced in the hippocampus. These changes indicate damage to the cytoskeleton. After PA, the presynaptic terminals, presynaptic vesicles and the ultra-structural organization of the PSDs were intact in the hippocampus. However, PA increased the thickness of the PSDs and ubiquitination of the synaptic proteins; and reduced mushroom-shaped dendritic spines (that contain F-actin). PA also reduced the amount of $\beta$-actin in synapses (Saraceno, Bertolino et al. 2010, Saraceno, Castilla et al. 2012). At 4.5 months after moderate PA (for $15 \mathrm{~min}$ ), there was a modest increase in glutamate kainate receptor binding in the CA1 layer (El-Khodor, Flores et al. 2004) The ceramides are components of the cell membrane and important regulators of cell activation. Eight months after PA (for $20 \mathrm{~min}$ ), there were marked differences in ceramide metabolism in the prefrontal cortex. Ceramide synthase 1 was reduced and sphingosine kinase 1 was increased by PA. Other ceramide metabolism enzymes and the inflammatory cytokine, TNF- $\alpha$, were not affected by PA (Vlassaks, Gavilanes et al. 2013). At 22 months, PA (for $20 \mathrm{~min}$ ) animals had an increased density of presynaptic boutons in the parietal cortex but not in the frontal cortex or the striatum (Van de Berg, Blokland et al. 2000).

Collectively, these studies show that PA causes long-term synaptic and signaling abnormalities in the hippocampus and other cognition structures. PA increased apoptosis in the developing hippocampus. However, this region also had increased neurogenesis and plasticity to compensate for the insult (Morales, Fiedler et al. 2008). The structures involved in cognition may be more resilient to PA than the locomotion-associated parts, and damage to these regions may be more reversible by the environmental influences across life. This could explain the inconsistent observation of cognitive deficits in this model, as discussed in section 3.3. Accordingly, life-long environmental enrichment improved cognitive performance in 18 month old rats with severe PA (Galeano, Blanco et al. 2014). 


\subsubsection{Growth factors:}

Growth factors play an important role in neurogenesis and neuronal repair after an insult. The majority of neurogenesis takes places prenatally, but in areas like the DG of the hippocampus, subventricular zone and olfactory bulb; this process continues throughout life. Lower levels of growth factors may contribute to the neuronal loss found after PA. The growth factors brain derived neurotrophic factor (BDNF) and neuroregulin-1 are also candidate genes for schizophrenia (Wakuda, Iwata et al. 2015). During the first 4 weeks after severe PA (for $20 \mathrm{~min}$ ), the amount of nerve growth factor (NGF) was reduced in the hippocampus, but not in the cerebellum. In this structure, the postnatal physiological increase in NGF at $\mathrm{P} 8$ was delayed. Brain derived neurotropic factor (BDNF) levels were lower than NGF levels and stay constant over time, but at P11 hippocampal BDNF was higher in PA animals than in controls. This is consistent with the increased regenerative capacity of the hippocampus. In the cerebellum, BDNF levels were reduced at P2, but similar to controls at P5. Also at P5, there was an increased neuronal proliferation within the hippocampus of PA animals, which was not mediated by BDNF or NGF (Scheepens, Wassink et al. 2003, Scheepens, Wassink et al. 2003).

One week after severe PA (for $20 \mathrm{~min}$ ) the levels of astrocytic basic fibroblast growth factor (bFGF) was increased in the whole hippocampus, accompanied by increased bFGF expression in the CA1 and CA2 and hilus layers. These changes persisted at 4 weeks after PA (Morales, Fiedler et al. 2008). 2 weeks after moderate PA (for $15 \mathrm{~min}$ ), the amount of bFGF was reduced in the SN/VTA. $\mathrm{bFGF}$ is a regulator of the $\mathrm{TH}$ gene and it is involved in the sensitization of the dopaminergic systems in response to amphetamine (Flores, Stewart et al. 2002). However, 4 weeks after PA (15-20 min), bFGF expression was increased in the SN/VTA (Andersson, Blum et al. 1995, Gross, Andersson et al. 2005). At 6 and 12 weeks after moderate PA (for $15 \mathrm{~min}$ ), BDNF levels in the prefrontal cortex and hippocampus were not altered by PA. The level of the synaptic trophic factor neuregulin-1 was significantly lower in the prefrontal cortex of the PA group at both time-points, but unaltered in the hippocampus (Wakuda, Iwata et al. 2015). At 12 weeks both C-section and moderate PA (for $15 \mathrm{~min}$ ) increased the binding site for the insulin-like growth factor I (IGF-I) and IGF-II receptors globally across all the hippocampal layers (Boksa, Zhang et al. 2006). Fourteen weeks after PA (for $15 \mathrm{~min}$ ) basal bFGF expression in the VTA was decreased and stress-induced bFGF release was increased in the VTA along with increased basal bFGF expression in the NAcc (Flores, Stewart et al. 2002). 


\subsubsection{Neuronal activation patterns; the histaminergic and adren- ergic system and nitric oxide signaling pathway:}

The $c$-fos gene is an immediate-early gene that is commonly used to visualize patterns of neuronal activity in the brain. Within 1 hour after the insult, the overall brain expression of $c$-fos is augmented. The overall turnover of noradrenalin was increased by 3 folds after 15-16 minutes of PA - and a decreased in animals exposed to 20-21 minutes of PA. In rats exposed to mild PA, c-fos mRNA was increased in the hippocampus (particularly the ventral medial area), neocortex, thalamus, striatum and nucleus tractus solitarius (NTS) of the brainstem. The induction of $c$-fos expression after PA was not mediated by catecholamines (Tang, Ringstedt et al. 2000). Eighty minutes after PA, c-fos-immunoreactivity (and thus presumably neuronal activity) in the piriform cortex was increased by short periods of PA (2-3 minutes) and decreased by longer periods of PA (more than 10 minutes). In the cortical amygdaloid complex, $c$-fos was strongly decreased after PA (for $20 \mathrm{~min}$ ) (Dell'Anna, Chen et al. 1995). At 2 months, male pups born with C-section had reduced noradrenalin levels in the amygdala and female pups had increased noradrenaline in the thalamus. In pups that had an additional PA (for $15 \mathrm{~min}$ ), the noradrenalin levels resembled those of the vaginally-born controls in both sexes - with the exception of reduced noradrenalin in the NAcc of PA female pups (El-Khodor and Boksa 2003).

At 3 months repeated cocaine administration increased the levels of $c$-fos in the NAcc of both rats that had PA and control animals. Moderate PA (for $15 \mathrm{~min}$ ) rats had a greater increase in $c$-fos than controls, whilst the cocaine-induced changes found in severe PA (for $20 \mathrm{~min}$ ) were smaller than in controls (Galeano, Romero et al. 2013). At the same age, severe PA (for 21 $\min )$, reduced the amount of $c$-fos-positive histaminergic neurons in the ventral tuberomammilary nucleus (the site of histamine production in the brain). This was accompanied by reduced expression of the histamine-synthesizing enzyme histidine decarboxylase in the hypothalamus. This may have additional implications for memory and learning (Flores-Balter, Cordova-Jadue et al. 2016) Collectively, the patterns of neuronal activation show that mild PA increased neuronal activity, whilst severe PA reduced activity. This mirrors the changes in locomotor behavior discussed earlier.

Nitric oxide (NO) is an important modulator of neuronal function. Physiological amounts of $\mathrm{NO}$ are required to maintain cerebral blood flow, but increased NO production results in the formation of reactive nitrogen species, like peroxynitrite, which worsens neurological damage (Keynes and Garthwaite 
2004). Shortly after mild to severe PA (5-20 min), when the fetal circulation and metabolism is still maintained, there were no apparent changes in the activity or transcription of neuronal nitric oxide synthase (nNOS) (Lubec, Kozlov et al. 1999) or inducible NOS (Calamandrei, Venerosi et al. 2004). At 5-10 days after severe PA (for $20 \mathrm{~min}$ ), the asphyxiated rats had increased production of $\mathrm{NO}$ in the striatum, observed as higher levels of $\mathrm{NO}$ /peroxynitrite and cyclic-GMP. Increased NO production was not seen in the cerebellum (Loidl, De Vente et al. 1998, Barkhuizen, Van de Berg et al. 2017). In 2 month old rats exposed to severe PA (for 19-20 min), changes in NO production was seen in the cervical region of the spinal cord neurons. In these regions, there were increases in inducible NOS, nitrotyrosine formation (indicator of reactive nitrogen species) and expression of nicotinamide adenine dinucleotide phosphate diaphorase (NADPH-d) - a marker of NO synthesis (Dorfman, ReyFunes et al. 2009). 6 month old rats exposed to severe PA (for 19-20 min) had increased NOS activity in the striatum and neocortex. NOS-containing neurons had ultra-structural changes indicative of neuronal damage the surrounding neurons showed increased degeneration (Capani, Loidl et al. 1997). In the same regions, neurons that express NADPH-d had similar ultra-structural abnormalities. The gross amount of NADPH-d expressing neurons was not altered by PA (Loidl, Capani et al. 1997).

\subsubsection{In vitro insights into neurocircuits:}

Organotypic cultures offer the opportunity to study neurocircuitry formation, since the procedure moves back development to an earlier stage. These cultures have provided insight into the alterations of various neurotransmitter systems after PA, including the NO synthesis pathways. Circuitry formation in several brain regions have been investigated with organotypic cultures made 3 days after PA (for $20 \mathrm{~min}$ ) and cultured for about a month before in vitro testing. These cultures showed that in control rats, the neocortex and striatum of control animals contained 5-fold more nNOS positive cells than the SN. In the PA group, the amount of nNOS positive cells increased by more than 5 -fold in the SN which was accompanied by less TH-immunoreactive cells, increased amounts of dead cells and shorter neurite lengths. Cell viability was not affected in the striatum or cortex. In the striatum, PA decreased nNOS positive cells and these cells also had shorter neurites, whilst there were no apparent changes in the cortex. Altogether these results show that the SN is more susceptible to PA than the striatum or cortex (Klawitter, Morales et al. 2006, Klawitter, Morales et al. 2007). Decreased dopaminergic innervation to niche areas of postnatal neurogenesis; like the DG of the hippocampus and 
the subventricular zone; has been associated with increased neurogenesis. This effect was investigated in several sets of organotypic cultures: a set isolated 7 days after PA (for $20 \mathrm{~min}$ ) and cultured for approximately a month and sets isolated 1-3 days after PA (for $21 \mathrm{~min}$ ) and cultured for 20-21 days. In these cultures, PA increased cell death within both the DG and SVZ. In both these regions, there was also a significant increase in cell proliferation and postnatal neurogenesis after PA. In the hippocampus, this effect was modulated through the dopaminergic D2 receptors (Morales, Huaiquin et al. 2007, Tapia-Bustos, Perez-Lobos et al. 2016).

\subsection{Translational aspects:}

\subsubsection{Strengths and weaknesses of this model:}

The perinatal-submersion model is an established rat model of birth injury in the very low gestational age (VLGA) human infant. The translational strengths of the perinatal submersion model are that it takes both the hormonal surges that occur during natural birth and the global nature of anoxia normally seen in the clinic into account (Boksa and El-Khodor 2003, Strackx, Van den Hove et al. 2010). Like in humans, the female rat pups have less neurological damage after PA than males (Loidl, Gavilanes et al. 2000, Calamandrei, Venerosi et al. 2004, Laplante, Brake et al. 2012) and the model can be adjusted to induce mild (5-10 min), moderate (15 min) and severe (19-22 min) PA (Van De Berg, Kwaijtaal et al. 2003, Capani, Saraceno et al. 2009). The effects of PA on neurotransmitter systems and behavioral outcomes vary according to the severity of asphyxia. A translational weakness is that, thus far, the majority of molecular studies have focused on grey matter structures and neurotransmitter systems. In humans, the sub-cortical white matter is the primary site of injury in the VLGA infant and grey matter damage occurs secondarily (Pierson, Folkerth et al. 2007, Logitharajah, Rutherford et al. 2009, Cabaj, Bekiesińska-Figatowska et al. 2012).

This model has been used to study both the acute short-term effects after PA; and long-term effects on neurotransmitter systems and behavior after insult at birth. Several groups have reported lasting alterations in dopaminergic signaling, anxiety, increased locomotion and increased sensitivity to stimulants after mild-moderate PA (see section 3.1, 3.2 and 4.1), which makes this model suited to study the neurodevelopmental origins of psychiatric diseases like schizophrenia (Boksa and El-Khodor 2003). Other groups have reported an exaggerated age-related cognitive decline in this model (Van de Berg, Blokland et al. 2000, Galeano, Blanco et al. 2014). It is not clear whether PA causes a 
single insult at birth with a lasting incomplete recovery, or whether this can trigger continual neurodegeneration which eventually leads to symptomatic impairments. Many adult-onset diseases have a long latency and models of progressive dysfunction are needed to understand the pre-symptomatic degenerative processes. Studies of the adult-onset deficits in this model provide some insights into this process, but combining this with additional developmental insults may better mimic the etiology of the adult-onset disorders (Marriott, Rojas-Mancilla et al. 2015). It has been proposed that several sporadic idiopathic adult-onset neurological diseases may have their onset due to damage during the early-developmental stages (Lahiri, Maloney et al. 2009, Marriott, Rojas-Mancilla et al. 2015). An early-life insult could alter the epigenetic regulation of neurodegenerative processes but remain asymptomatic until an individual is exposed to a second insult (or several insults) later in life. Collectively these insults would cause enough pathology to manifest as neurological diseases like schizophrenia or Alzheimer's disease (Lahiri, Maloney et al. 2009).

This model has been combined with a fetal mild hypoxic insult at gestational day 17. Those studies have shown that the addition of an earlier hypoxic insult can attenuate the acute cytokine response (Vlassaks, Strackx et al. 2013), reduce apoptosis and astrogliosis (Cox-Limpens, Strackx et al. 2015) and alter the transcriptional response through changes in epigenetic regulation (CoxLimpens, Vles et al. 2014) in neonatal rats through the biological phenomenon of preconditioning to the insult. The effects of fetal preconditioning were still evident in adult rats through reduced locomotor and cognitive impairment (Strackx, Van den Hove et al. 2010) and altered ceramide metabolism and inflammatory cytokines (Vlassaks, Gavilanes et al. 2013) in response to PA. The effect of maternal anesthetic use in combination with PA has also been studied in this model (Vaillancourt and Boksa 1998). However, studies which combine PA with an additional postnatal insult are lacking and they could provide valuable insights into progressive neurological dysfunction (Marriott, Rojas-Mancilla et al. 2015).

The strengths of this model are discussed above. This model is highly reproducible between laboratories. However, there are subtle variations between methodologies which may influence the results. The use and choice of anesthetic to the dam before removal of the uterine horns may influence several neurological parameters, as anesthetics may cross the placental barrier. In this model, it has been shown that maternal anesthesia with isoflurane $/ \mathrm{N}_{2} \mathrm{O}$ resulted in greater amphetamine sensitization was found in the pups (Vaillancourt and Boksa 1998). Some groups sacrificed the mother by rapid decapitation 
without any anesthesia (Van de Berg, Schmitz et al. 2002, Vlassaks, Brudek et al. 2014), under $\mathrm{CO}_{2} / \mathrm{O}_{2}$ anesthesia (Grimaldi, Romer et al. 2012) or under pentobarbital anesthesia (Herrera, Romero et al. 2014) whilst others used cervical dislocation under anesthesia with isoflurane (Klawitter, Morales et al. 2007). The timing of the procedure also varied from initiation after normal vaginal birth of the first pup (Van de Berg, Schmitz et al. 2002, Grimaldi, Romer et al. 2012, Vlassaks, Brudek et al. 2014) or within the last day of gestation (Tapia-Bustos, Perez-Lobos et al. 2016). Another weakness refers to the grading of asphyxia as mild, moderate or severe based solely on the submersion time. Among the durations of submersion used in severe asphyxia, there are large variations in the percentage of each litter which survived the insult (i.e. 35\%-60\% survival after 19-20 minutes (Loidl, Gavilanes et al. 2000, Klawitter, Morales et al. 2007, Dorfman, Rey-Funes et al. 2009) and 10-70\% survival after 21 minutes of submersion (Loidl, Gavilanes et al. 2000, TapiaBustos, Perez-Lobos et al. 2016). The inclusion of survival percentages and rat Apgar scores (Chen, Engidawork et al. 1997, Dell'Anna, Chen et al. 1997, Marriott, Rojas-Mancilla et al. 2015) can harmonize the grading of asphyxia across studies. The lack of a clear criterion may contribute to contradicting results found between laboratories.

\subsubsection{Other models of PA:}

There are various other animal models available to study PA. Non-human primates are arguably the best model of human functioning and have been used in biomarker research for PA (Traudt, McPherson et al. 2013, Chun, McPherson et al. 2015). However, ethical and cost concerns prevent large-scale use of these models. Ovine and porcine models represent other key large-animal models of asphyxia. These models have the advantage that the majority of brain development occurs prenatally, like in humans. Asphyxia can be induced at several gestational ages through instrumentation of the fetal pigs/sheep. These models provide a stable thermal and biochemical excellent to study the acute molecular and physiological effects of PA and therapies (Mueller-Burke, Koehler et al. 2008, Seehase, Jellema et al. 2011, van den Heuij, Wassink et al. 2016). However, the longer lifespan of large animals; and reduced amount of standardized behavioral tests; makes rodents key for the study of functional outcomes after PA.

There are variations on modeling birth asphyxia in rats. Traditional models of neonatal brain injury had used 7-10 day old rat pups. At this time-point, neurodevelopmental milestones in the rat resemble those of the human fetus 
from week 36-40 (Vannucci and Vannucci 2005, Semple, Blomgren et al. 2013). The most commonly used model of injury in the late-preterm neonate is the Rice-Vannucci model. In this model, 7-day old rats undergo unilateral carotid artery ligation, accompanied by hypoxia (inhalation of $8 \% \mathrm{O}_{2}$ ) for 20 minutes to 3.5 hours (Vannucci and Vannucci 2005). This model was originally derived from the Levine model, which used adult rats. Unilateral carotid artery occlusion causes one-sided focal brain damage, which models neonatal stroke rather than global HIE. Another method uses anoxic $\mathrm{N}_{2}$ inhalation, first described by Speiser, Korczyn et al. (1983). Neonatal rat pups were exposed to $100 \% \mathrm{~N}_{2}$ for 20-25 minutes within 24-48 hours after birth. This method represents global anoxia and caused persistent changes in the brain's neurochemical systems. However, the morphological changes readily seen in human infants, were not detectable (Dell'anna, Luthman et al. 1993).

\subsubsection{Therapeutic developments:}

From this model, various therapeutic strategies had emerged. An in-depth discussion of all of these studies was beyond the scope of this review. Currently, therapeutic hypothermia - either globally or with selective head cooling - is the only intervention used in the clinic for human term infants (Azzopardi, Strohm et al. 2014). Preliminary results in preterm infants indicated that the therapy seems feasible in human infants born at 34-35 weeks of gestation, but it may increase the risk of mortality and adverse effects in this age group (Rao, Trivedi et al. 2016). It did not prevent mortality or disability in a small pilot trial of infants born at 32-35 weeks of gestation (Walsh, Butler et al. 2015), but the two studies in preterm infants were underpowered for this end-point.

Many pre-clinical studies on the benefit of hypothermia have been done with this model and the majority of them have found therapeutic benefits (see (Herrera-Marschitz, Loidl et al. 1993, Capani, Loidl et al. 1997, Loidl, Capani et al. 1997, Loidl, Gavilanes et al. 2000, Engidawork, Loidl et al. 2001, Capani, Loidl et al. 2003, Cebral, Capani et al. 2006, Capani, Saraceno et al. 2009, Cebral and Loidl 2011, Muñiz, Romero et al. 2014) and many others. Whilst preclinical studies have shown hypothermia could reduce injury in preterm infants too, this group has historically reported more adverse effects after cooling. The risk of cooling may outweigh the benefits in preterm - and VLGA - infants and it is currently not recommended outside of the clinical trial setting. Any new therapeutic developments should first show efficacy in term and older preterm infants before being applied to preterm and VLGA infants in clinical trials (Gunn and Bennet 2008). 
Several other potentially therapeutic strategies have been tested in preclinical models and are now in phase I/II clinical trials. These include xenon, melatonin, erythropoietin, allopurinol, magnesium sulphate, topiramate and autologous umbilical cord stem cells. Additional therapies which may progress to clinical trials soon include: N-acetylcysteine, peptides which mimic erythropoietin and resveratrol given postnataly to infants that already have mild-severe $\mathrm{HIE}$; and tetrahydrobiopterin, nNOS inhibitors, vitamin $\mathrm{C}$ and vitamin $\mathrm{E}$ given antenataly to a mother in whom fetal distress is detected (reviewed by (Buonocore, Perrone et al. 2012, Robertson, Tan et al. 2012, Wu, Chen et al. 2015)). Since therapeutic hypothermia is already well-established in the clinic for term infants, it is important that new putative therapies show additive benefit when combined with hypothermia. Agents which share mechanisms of action with hypothermia may not show any cumulative benefit when given as a combination therapy. Preclinical work has shown that melatonin, stem cells and xenon have additive benefits in combination with hypothermia, whilst strategies combining erythropoietin, anticonvulsants, anti-apoptotic factors and blockade of connexin hemichannels showed no additional benefit (reviewed by (Davidson, Wassink et al. 2015)).

Various other pharmacological and non-pharmacologic interventions that are not (or not yet) in clinical trials have also been tested in this model. Sentinel proteins, like PARP-1, are activated upon DNA damage. PARP-1 competes with NAD+ during the reoxygenation period and it may be a viable target in PA (Herrera-Marschitz, Morales et al. 2011). The PARP-1 inhibitor nicotinamide has been extensively studied in this model and shown to have beneficial effects on the cellular, neurochemical and behavioral consequences of PA (Bustamante, Goiny et al. 2003, Klawitter, Morales et al. 2006, Bustamante, Morales et al. 2007, Morales, Simola et al. 2010, Allende-Castro, Espina-Marchant et al. 2012, Neira-Peña, Rojas-Mancilla et al. 2015). PARP-1 inhibition as a therapeutic strategy is extensively discussed in reviews by (Herrera-Marschitz, Morales et al. 2011, Herrera-Marschitz, Neira-Peña et al. 2015).

Other studies have investigated glutamate antagonism for modest survival benefits (Herrera-Marschitz, Loidl et al. 1993, Engidawork, Loidl et al. 2001) and the behavioral benefits of the cholinergic agonist nicotine (Chen, Bjelke et al. 1995); the alpha-type peroxisome proliferators activated receptor (PPAR- $\alpha$ ) agonist oleoylethanolamide (Herrera, Romero et al. 2014); bio-active marine-derived peptides (Xu, Dong et al. 2015) and a histamine-3-antagonist (Flores-Balter, Cordova-Jadue et al. 2016). Dopamine-receptor antagonism 
has been investigated in vitro for cellular benefit in this model (Tapia-Bustos, Perez-Lobos et al. 2016). All of the compounds listed above have shown a modest beneficial effect on either a molecular parameter or a behavioral outcome, but more studies are needed to determine whether these compounds show a lasting global beneficial effect to the scale that warrants further clinical development.

Non-pharmacological interventions have also been studied in this model. Of note, increased environmental stimulation attenuated the neurodevelopmental delay due to PA and reduced cognitive deficits in middle age rats (Kiss, Vadasz et al. 2013, Galeano, Blanco et al. 2014). This intervention is feasible, non-invasive and implementable in the daily life of children with PA. Finally, a number of studies have investigated preconditioning in this model. Our group has investigated preconditioning due to mild fetal asphyxia (Strackx, Van den Hove et al. 2010, Strackx, Zoer et al. 2010, Cox, Strackx et al. 2011, Vlassaks, Gavilanes et al. 2013, Vlassaks, Strackx et al. 2013, Cox-Limpens, Strackx et al. 2015). Others have investigated the preconditioning effects of low-doses inhalation anesthetics in this model (Yang, Zhuang et al. 2012). Whilst preconditioning is not directly translatable to a therapy, these studies have provided mechanistic insights into neuroprotection which can be exploited in selecting agents for future preclinical investigations.

\subsection{Discussion and conclusion:}

In the past 25 years, many strides towards understanding and treating global asphyxia have been made with the perinatal-submersion model. From this review, it becomes clear that global PA affects multiple organs; all CNS regions including brain, cerebellum and spinal cord; on a molecular and behavioral level.

The majority of the research in this model has focused on grey matter structures in the basal ganglia and the hippocampus. From these results, a dose-effect ratio of PA emerged. In the majority of studies, mild asphyxia increased neurotransmitter release in these systems, accompanied by ADHD-like behavior. Severe asphyxia decreased neuronal activation. This was accompanied by diminished locomotor function, cognitive deficits and anxiety. Thus, the length and severity of asphyxia may determine whether a hyperactive clinical phenotype (like ADHD and schizophrenia) or severe functional impairment (like cerebral palsy) is observed in the clinic. In the basal ganglia, the GABAergic neurons were especially sensitive to PA. These neurons inhibit excessive dopaminergic outflow. From a behavioral perspective, asphyxiated animals had 
increased anxiety across the majority of experiments and locomotor dysfunction in experiments which studied longer durations of asphyxia. These animals also had social deficits and poor maternal instincts. It appears that PA did not affect the serotonergic system and depressive symptoms later in life, but this neurotransmitter system is understudied in this model. Reports on cognitive impairment after PA are been mixed. Most studies only found slight memory deficits in rats that were older than 4 months. PA exaggerated age-related memory decline and studies that were performed from 17-20 months found more convincing deficits. However, recently the Capani group already found impairment in the domains of recognition, spatial and reference memory but an increased habituation memory (Blanco, Galeano et al. 2015, Saraceno, Caceres et al. 2016).

A vast number of pathological proteins and processes had been identified in this model. Both short- and long-term consequences - ranging from increased apoptosis, oxidative stress and inflammatory cytokines after primary energy failure to long-term alterations in the post-synaptic terminals had been identified in this model. Earlier studies evaluated the expression of candidate genes and proteins, whilst newer studies have used transcriptome-wide approaches to untangle the biochemical effects of PA. From all of these studies, it was clear that PA affects a multitude of cellular processes (cell nucleus function, cell signaling and cellular stress) simultaneously. Thus, although many single pharmacological agents reduce some of the damage seen after PA, we need therapies that target many pathological processes simultaneously. A good example of this is therapeutic hypothermia, currently still the only FDA-approved therapy for PA in the term infant.

Although this model had greatly improved our understanding of PA, there are several areas left to explore. In particular, the serotonergic system and subcortical white-matter damage have largely been neglected in this model. The majority of studies focused on acute changes, whilst the challenges faced by VLGA PA infants throughout life are of crucial importance. The race to find an effective therapeutic option for premature infants is still on, but we have already jumped out of the starting blocks towards a remedy. 


\subsection{Acknowledgements:}

This research was partially supported by the Sistema de Investigación y Desarrollo (SINDE) of the Universidad Católica de Santiago de Guayaquil, Guayaquil, Ecuador, through the grant: Perinatal asphyxia and stem cell treatment. M. Barkhuizen is funded by the National Research Foundation of South Africa (Grant specific reference number 89230 and 98217) and the Foundation of Pediatrics, Maastricht University Medical Center + . All views expressed in this article are those of the authors and not of the funding agencies. 




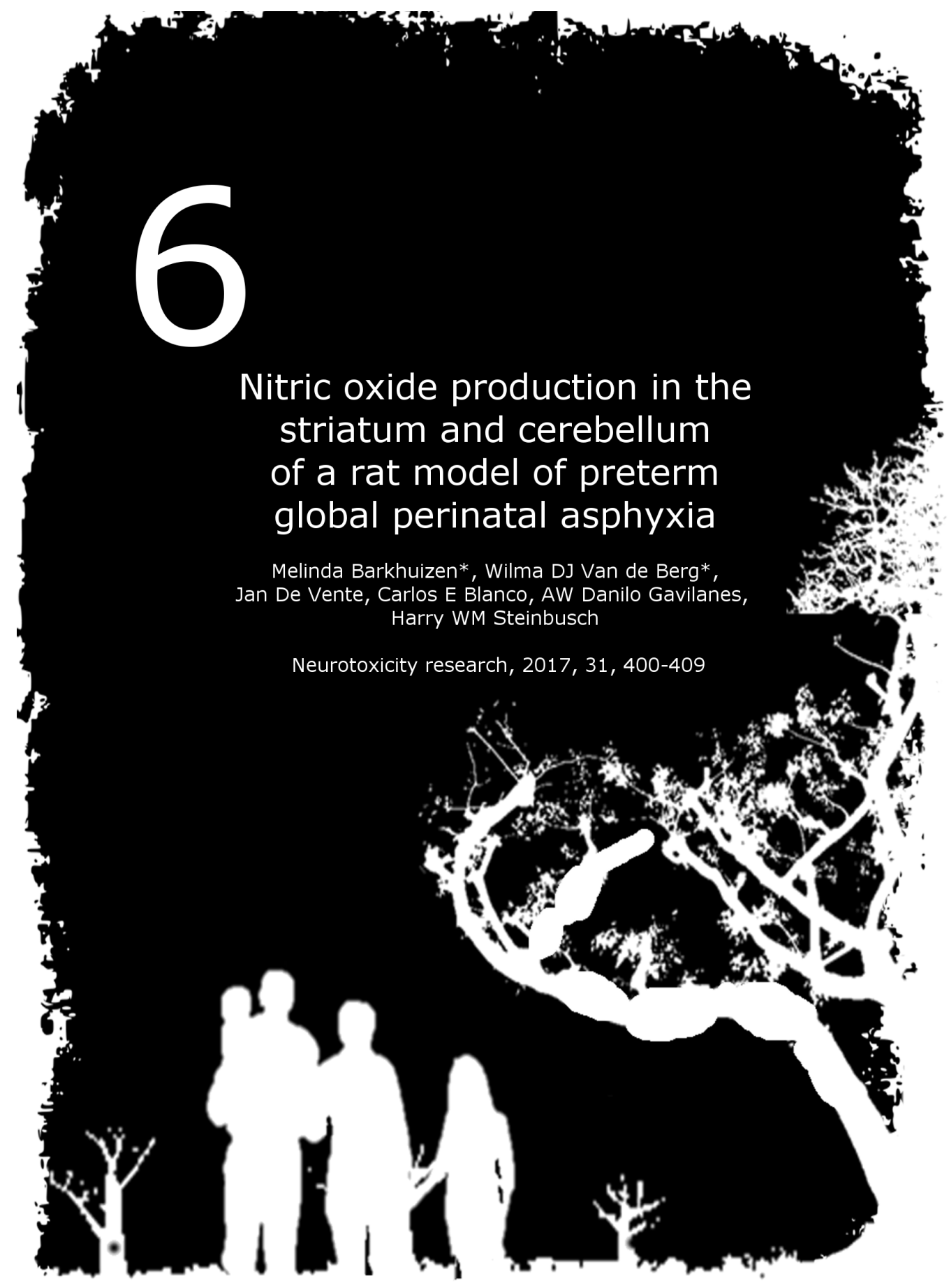




\title{
Nitric oxide production in the striatum and cerebellum of a rat model of preterm global perinatal asphyxia
}

Melinda Barkhuizen*, Wilma DJ Van de Berg*, Jan De Vente, Carlos E Blanco, AW Danilo Gavilanes, Harry WM. Steinbusch

* These authors contributed equally

Neurotoxicity research, 2017, 31, 400-409

\begin{abstract}
:
Encephalopathy due to perinatal asphyxia (PA) is a major cause of neonatal morbidity and mortality in the period around birth. Preterm infants are especially at-risk for cognitive, attention and motor impairments. Therapy for this subgroup is limited to supportive care and new targets are thus urgently needed. Post-asphyxic excitotoxicity is partially mediated by excessive nitric oxide (NO) release. The aims of this study were to determine the timing- and distribution of nitric oxide (NO) production and apoptosis in brain regions involved implicated in motor deficits after global PA. This study focused on the rat striatum and cerebellum, as these areas also affect cognition or attention, in addition to their central role in motor control. NO/peroxynitrite levels were determined empirically with a fluorescent marker on postnatal days P5, P8 and P12. The distributions of neuronal NO synthase (nNOS), cyclic guanosine monophosphate (cGMP), astroglia and caspase-3 were determined with immunohistochemistry. Apoptosis was additionally assessed by measuring caspase-3-like activity from P2-P15. On P5 and P8, increased intensity of NO-associated fluorescence and cGMP-immunoreactivity after PA was apparent in the striatum, but not in the cerebellum. No changes in nNOS-immunoreactivity or astrocytes were observed. Modest changes in caspase-3-activity were observed between groups, but the overall time-course of apoptosis over the first 11 days of life was similar between PA and controls. Altogether, these data suggest that PA increases NO/peroxynitrite levels during the first week after birth within the striatum, but not within the cerebellum, without marked astrogliosis. Therapeutic benefits of interventions that reduce endogenous NO-production would likely be greater during this time-frame.
\end{abstract}

Keywords: Asphyxia; nitrosidative stress; cGMP; neuronal nitric oxide synthase; peroxynitrite; selective vulnerability 


\subsection{Introduction:}

Neonatal encephalopathy (NE) due to perinatal asphyxia (PA) is a common cause of morbidity and mortality in the period around birth. In 2010, 8.5 infants per 1000 live term-births developed PA-related encephalopathy (Lee, Kozuki et al. 2013). The long-term neurological outcome after NE varies, from normal neurocognitive functioning after mild asphyxia to cerebral palsy, epilepsy, cognitive, behavioural or memory problems in severe cases (Armstrong-Wells, Bernard et al. 2010, Volpe 2012). PA-related injury to the preterm brain is even more complex, since prematurity by itself increases the risk of NE (Volpe 2009a). While preterm birth before 37 weeks of gestation occurs in 5-8\% of all pregnancies, very low gestational age (VLGA) before 32 weeks of gestation occurs in about $1 \%$ of singletons and $9 \%$ of twin pregnancies (Schaaf, Mol et al. 2011). In cohorts of preterm infants with multifactorial encephalopathy, behavioural, cognitive, attention or social deficits have been reported in 25-50\% of cases and $5-10 \%$ had major motor impairments. A large portion of this disability burden was made up by VLGA infants, due to improved survival rates with modern medical care. Although the major motor impairments are striking, cognitive deficits are far more common (Volpe 2009a, Volpe 2009c). In preterm infants, PA causes both white-matter injury of the developing oligodendrocytes (periventricular leukomalacia) in the sub-cortical regions and associated grey matter injury to the striatum and other basal ganglia structures, thalamus, basis pontis, brain stem and cerebellum (Shah, Anderson et al. 2006, Logitharajah, Rutherford et al. 2009, Cabaj, Bekiesińska-Figatowska et al. 2012). Injury in these infants is a combination of primary destruction after PA and secondary maturational and trophic disease (Volpe 2009c).

PA occurs when oxygen supply between the mother and the fetus is disrupted, causing a bi-phasic brain-injury. The acute injury results from the combined effects of cellular energy failure, acidosis, glutamate release, intracellular calcium accumulation, lipid peroxidation, and nitric oxide (NO) neurotoxicity that disturb vital cell components. This results in cell death. From 6-48 hours after the insult, a secondary cerebral energy failure occurs with mitochondrial dysfunction due to sustained pathological reactions in the primary phase (Perlman 2006, Piña-Crespo, Sanz-Blasco et al. 2014). The time-window for therapeutic intervention is thus limited. Currently, therapeutic hypothermia is the only therapy available for term-infants with NE. This only partially reduces morbidity and it is not used in pre-term infants (Edwards, Brocklehurst et al. 2010). There is thus an urgent need for therapies in the preterm- and term infant. Animal models are essential tools for the preclinical development and testing of new therapies. In humans and large-animals, the 
majority of brain-development occurs prenatally. However, in rodents, the brain is still immature at birth and only resembles the term human infant at roughly 7-10 days postnatal (Semple, Blomgren et al. 2013). This also makes the rat a suitable organism to study insults to the preterm brain. Our group, and others, have optimized a rat model of global anoxia during birth to investigate PA in the rodent-equivalent of the 23-32 week old human fetus (Semple, Blomgren et al. 2013).

In this study, we focused on two structures important for motor control and coordination, which are in differential developing states at the time of insult, namely the striatum and the cerebellum. The primary roles of the striatum involve learning of associations between stimuli, actions and rewards (Balleine, Delgado et al. 2007), the selection between competing response alternatives; and motivational modulation of motor behaviour (Liljeholm and O'Doherty 2012, Lenz and Lobo 2013). In humans, neurogenesis of the striatal medium spiny neurons begins around week 11.5 and striatal synaptogenesis begins around 13 weeks of gestation. Synaptogenesis is well-established before mid-gestation, with near-uniform synapses by 34 weeks of human gestation (Freeman, Sanberg et al. 1995, Sarnat, Auer et al. 2013). The cerebellum undergoes rapid expansion from week 24 to 40 of gestation. During this time, the cerebellar volume increases by $3.5-5$ fold. The cerebellum is particularly vulnerable in preterm infants, due to its rapid growth towards the end of gestation (Volpe 2009b). The cortico-striato-cerebellar tract is instrumental for motor sequence learning (Tzvi, Münte et al. 2014). In addition to its established role in motor coordination, the cerebellum also directs linguistic and related cognitive and behavioral-affective functions (De Smet, Paquier et al. 2013). Damage to these two regions are of clinical significance for both the motor deficits and cognitive/attention deficits in after PA (Volpe 2009b, Volpe 2012).

The NO-cascade has emerged as both a major player in neurotoxicity and a potential therapeutic intervention in PA. During PA, NO is involved in both the early phase injury and during secondary energy failure. Immediately after asphyxia at birth neuronal nitric oxide synthase (nNOS) is activated, increasing NO neurotransmission. After 12-24 hours, inducible nitric oxide synthase (iNOS) is activated in glial cells which leads to cerebral NO production (Perlman 2006, Gunes, Ozturk et al. 2007). Concurrent increases in the generation of superoxide cause the formation of peroxynitrite $\left(\mathrm{ONOO}^{-}\right)$. Peroxynitrite is a potent oxidative agent which causes tissue injury and contributes to ischemic injury in the immature brain, by irreversibly inhibiting 
the mitochondrial respiratory chain (Ikeno, Nagata et al. 2000, Weis, Schunck et al. 2011). The new-born brain is especially vulnerable to this type of insult due to its relatively low antioxidant levels (McQuillen and Ferriero 2004, Shim and Kim 2013). The extent of excessive NO production is dependent on the severity/duration of asphyxia, with variability across different neuron types and nervous system locations (Klawitter, Morales et al. 2007, Dorfman, Rey-Funes et al. 2009). Nitrotyrosine - a product of peroxynitrite and proteins - was present in the brain tissue of human NE infants at autopsy (Groenendaal, Lammers et al. 2006). Moreover, increased NO in the cerebrospinal fluid in the first 24 hours correlates with the severity of the NE (Ergenekon, Gücüyener et al. 2004, Gunes, Ozturk et al. 2007). The exact role of NO and peroxynitrite in mediating the effects of PA remain to be elucidated. Pharmacological inhibition of neuronal nitric oxide synthase (nNOS) has been proposed as a strategy to reduce cerebral palsy and other motor deficits after PA $(\mathrm{Ji}$, Tan et al. 2009, Yu, Derrick et al. 2011) and nNOS inhibitors potent neuroprotective agents in most animal model for hypoxia-ischemia and excitotoxicity in vitro. Selective inhibition of nNOS shows beneficial effects including preservation of mitochondrial function after in utero ischemia. nNOS inhibition also improves the short-term survival of GABAergic interneurons in the striatum in hypoxic ischemic preterm sheep and delays the onset of post-asphyxial seizures (Rao, Lin et al. 2011, Drury, Davidson et al. 2014). Inhaled NO has shown beneficial effects in rodent models of neonatal stroke. However, high doses of NO, or low doses of NO administered during the reperfusion period are detrimental (Charriaut-Marlangue, Bonnin et al. 2012).

The aim of this study was to determine the timing and regional-selectivity of increased NO production after global PA in motor regulation and coordination structures. Targeting supra-physiological NO-production could be a fertile therapeutic target for the preterm infant. In order to use NO-production for a therapeutic target, we need to know at which time-points after PA production peaks, whether these peaks coincide with increased cell death and whether regional differences exist in the brain. The focus of this study was the striatum and cerebellum. 


\subsection{Materials and methods:}

\subsubsection{Animals:}

Full-term pregnant Wistar rats (Charles River-Broekmans, Someren, The Netherlands) and their male pups $(\mathrm{n}=108)$ were used for the present study. Female pups were sacrificed at birth. The pups were divided into two groups (PA and control). 6 pups per group were used at each time-point (fig. 6.1). They were housed under standard conditions (12:12 h light: dark cycles, 20 ${ }^{\circ} \mathrm{C}$ ) with free access to standard laboratory chow and water. The local Committee on Animal Welfare approved all animal care and procedures. Within this study, exclusively male offspring were used because both morphological and behavioural studies provided evidence for a differential vulnerability to a birth insult in males versus females. A greater impact is seen in the male sex, probably due to the protecting role of the circulating hormones in females (El-Khodor and Boksa 2003, Zhu, Xu et al. 2006).

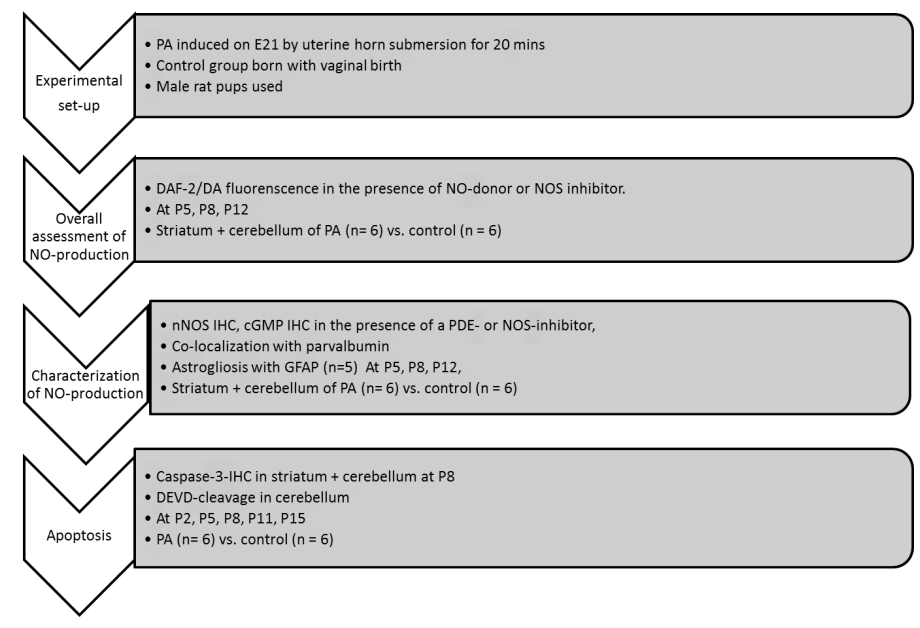

Figure 6.1: The study design

\subsubsection{Induction of PA:}

PA was induced in rat pups at P0 by placing the uteri and its contents in a water bath for 20 minutes, as described previously (Vlassaks, Brudek et al. 2014). Briefly, time-pregnant Wistar dams were decapitated immediately after delivery of two pups (control vaginal deliveries) and rapidly hysterectomized. The uterus horns containing the remaining pups were placed in a water bath at $37^{\circ} \mathrm{C}$ for 20 min (severe PA). Afterwards, pups were removed from the uterus horns and stimulated to breath by cleaning their skin and by gently padding 
them on the chest. The pups were left to recover for $60 \mathrm{~min}$ in a pediatric incubator at $36.5^{\circ} \mathrm{C}$ and randomly assigned to a surrogate mother (10 pups per mother), which had given birth on the same day. The percentage of mortality in the PA and control group was respectively $\pm 50 \%$ and $0 \%$.

\subsubsection{4,5-Diaminofluorescein diacetate (DAF-2/DA) detection in slices:}

For the DAF2/DA detection in fresh tissue, 6 rat pups were decapitated and their brains were rapidly removed and placed into ice-cold Krebs-Ringer bicarbonate buffer ( $\mathrm{pH} 7.4$ ) aerated with $95 \% \mathrm{O}_{2} / 5 \% \mathrm{CO}_{2}$. The forebrain and the cerebellum were chopped into $300 \mu \mathrm{m}$ coronal slices using a McIllwain tissue chopper. Slices were separated under a microscope and transferred to multi-well culture plate containing Krebs buffer $\left(4^{\circ} \mathrm{C}\right.$; $\mathrm{pH}$ 7.4) and $1 \mathrm{mM}$ isobutyl-methyl xanthine (IBMX) to inhibit 3,5'-cyclic nucleotide phosphodiesterase (PDE) activity. Alternated slices were transferred to a second multi-well plate and used for nNOS and cGMP immunohistochemistry. All slices were incubated in Krebs-Ringer buffer containing IBMX for $30 \mathrm{~min}$ and slowly warmed to $35.5^{\circ} \mathrm{C}$ or $25.5^{\circ} \mathrm{C}$ under an atmosphere of $5 \% \mathrm{CO}_{2} / 95 \% \mathrm{O}_{2}$ prior to DAF2/DA staining.

DAF-2-triazole (DAF-2/T) fluorescence was studied in slices $(300 \mu \mathrm{m})$ including the striatum of rat pups at postnatal day P5, P8 and P12 (n =6 per group at each time-point) using the DAF2/DA detection assay (Sigma, The Netherlands) according to the method of Lopez-Figueroa, Caamano et al. (2000). Upon entry into the cell, DAF-2/DA is hydrolysed by cytosolic esterases, producing DAF-2. DAF-2 reacts with $\mathrm{NO}$ and peroxynitrite to form the highly fluorescent derivative DAF-2/T (Roychowdhury, Luthe et al. 2002, Rodriguez, Specian et al. 2005, Bryan and Grisham 2007). The slices were incubated with $1.5 \mathrm{ml} 10 \mu \mathrm{M}$ DAF-2/DA incubation buffer (150mM Tris-HCl, $3 \mu \mathrm{M}$ tetrahydrobiopterin, $1 \mu \mathrm{M}$ flavin adenine dinucleotide (FAD, Sigma), $1 \mu \mathrm{M}$ flavin mononucleotide (FMN, Sigma), 1mM NADPH, 0.6mM CaCl 2 (Merck) and $100 \mu \mathrm{M}$ L-arginine (Sigma) per well for $45 \mathrm{~min}$ at $35.5{ }^{\circ} \mathrm{C}$ or $25.5^{\circ} \mathrm{C}$. During incubation with the DAF-2/DA solution, lights were turned off and a dark box was placed over the incubation chamber. Following incubation with DAF-2/DA, the slices were washed in phosphate-buffered saline (pH 7.4) and placed on non-coated slides and cover slipped using PBS-glycerol (1:4).To confirm that DAF-2/DA has a high affinity for NO, slices were incubated in the presence or absence of the NO donor $0.1 \mathrm{mM}$ sodium nitroprusside (SNP; Sigma) for $10 \mathrm{~min}$, or the NOS inhibitor $0.1 \mathrm{mM} N^{G}$-nitro-L-arginine (Sigma) for $30 \mathrm{~min}$. As a negative control, slices were incubated in media lacking 
DAF-2/DA.

\subsection{4 nNOS, cGMP and caspase-3 immunohistochemistry:}

Slices of the striatum and cerebellum $(\mathrm{n}=6,10 \mu \mathrm{m}$ thick sections adjacent to the slices used in the DAF-2/DA experiment) were used to visualize cGMPproducing and NOS active structures, using antisera against cGMP (de Vente, Steinbusch et al. 1987) and nNOS (Herbison, Simonian et al. 1996). After incubation in the presence or absence of SNP or $N^{G}$-nitro-L-arginine, slices were fixed for 2 hours with $4 \%$ paraformaldehyde and cut into were with a cryostat. The sheep anti-cGMP and anti-nNOS antisera were used respectively at a dilution of 1:4,000 and 1:2,000 and visualized using an Alexa-conjugated donkey anti-sheep antibody (1:100; Mol. Probes, USA). The nNOS and cGMP sections were processed to study co-localization with cell-specific markers. An antibody against glial fibrillary acidic protein (anti-GFAP) (1:1600; Sigma) was used to identify astrocytes $(\mathrm{n}=5)$. The immunolabelling was visualized with a Cy3-conjugated donkey anti-mouse antiserum (1:800) or goat anti-mouse Alexa Fluor@ 488 (1:100; Mol. Probes, USA). The sections were incubated overnight at $4{ }^{\circ} \mathrm{C}$ with the primary antibody followed by 2 hours at room temperature with the secondary antibody. Negative controls were performed by omitting the primary antibody. nNOS immunoreactivity labelled low-threshold spiking interneurons in the striatum and the cerebellar granule cells. Parvalbumin immunohistochemistry identified the fast-spiking striatal interneurons and cerebellar Purkinje cells. Basket and stellate interneurons in cerebellar molecular layer express both parvalbumin and nNOS (Schwaller, Meyer et al. 2002, Contestabile 2012, Lenz and Lobo 2013). Parvalbumin-immunoreactivity ( $\mathrm{n}=$ 6) was visualized with a rabbit antiserum (1:1500) provided by P.C. Emson (Babraham Institute, Cambridge, UK) in combination with a donkey antirabbit biotinylated antiserum (1:400) and streptavidine-Cy3 (1:2000). Sections were cover slipped using TBS: glycerol (1:3). Sections were examined at a magnification of x400 and x1000 with an Olympus AX-70 microscope.

For caspase-3 immunohistochemistry, another set of 6 pups were anaesthetized with sodium pentobarbital and perfused transcardially with a fixative containing $4 \%$ paraformaldehyde and $2 \%$ picric acid. Afterwards, the brains were snap-frozen and cut into $16 \mu \mathrm{m}$ thick coronal sections. These sections were incubated with a rabbit polyclonal anti-caspase-3 antibody (67341A; Pharmingen, Europe) diluted 1:500. The anti-caspase-3 antibody was visualized using a biotinylated goat anti-rabbit antibody (1:400; Jackson Immunoresearch Laboratories) and streptavidine Cy-3. Caspase- 3 positive cells were counted in 
tissue of six rats of each group (PA and control) at P8 as described previously in Van de Berg, Schmitz et al. (2002). The total amount of caspase-3-positive cells was estimated by multiplying the number of counted cells in all sections by the sampling interval (i.e., equal to eight).

\subsubsection{Fluorometric assay of caspase-3-like activity:}

Another set of 6 control and 6 asphyctic rat pups was used for analyses of caspase-3-like activity at P2, P5 or P8, P11 and P15 in cerebellum homogenates. The cerebellum was collected and homogenized in a lysis buffer containing $137 \mathrm{mM} \mathrm{NaCl}, 20 \mathrm{mM}$ Tris- $\mathrm{HCl}$ (pH 8.0), $1 \%$ NP-40, $10 \%$ glycerol and a complete protease inhibitor tablet (Roche, NL). The tissue samples were briefly centrifuged and an aliquot of the supernatant (30 or $50 \mu \mathrm{l})$ was used. The assay is based on fluorometric determination of the cleavage of the Ac-Asp-Glu-Val-Asp-AMC peptide (Ac-DEVD-AMC; Biomol, Germany) by caspase-3 as described in detail previously (Van den Hove, Steinbusch et al. 2006). The cleavage was followed at 2 minute intervals for 3 hours.

\subsubsection{Image analysis:}

Fluorescence intensity of the images obtained from the slices loaded with DAF-2/DA and immunostained sections were analyzed using a macro designed for measuring grey values in a given area (region of interest, ROI). Background values were measured using the slices incubated with the incubation buffer lacking the DAF-2/DA or lacking the primary antibody. Measurements were performed on 3 sampled images of 3 systematically sampled slices. From these data, the mean grey value in the area was calculated. Digital images were captured using a CoolView CCD camera system attached to a MR C600 confocal microscopy (Leica Microsystems, Germany). The microscope was equipped with a narrowband MNIBA-type FITC filter, or MNG filter for CY fluorescence (Chroma Technology, The Netherlands). Excitation was measured at $488 \mathrm{~nm}$ and emission at $530 \mathrm{~nm}$. Grey scaled images were directly converted into

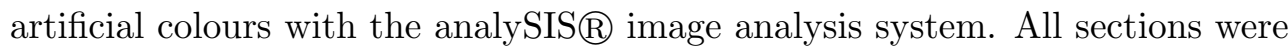
stained simultaneously and recorded on the same day to minimize experimental variation. 


\subsubsection{Statistics:}

Group comparison (controls vs. PA) of the number and caspase-3 positive cells as well as the grey values of immunofluorescence intensity measurements were analysed using the Student's $t$-test. The differences in Ac-DEVD-AMC cleavage between control and asphyctic rats were evaluated using a pairwise two-way ANOVA analysis and post-hoc tests using Bonferroni correction for repeated measures. Statistics were carried out using SigmaStat ${ }^{T M}$ software version 2.03. Differences were considered significant if $\mathrm{P} \leq 0.05$. All data are presented as mean \pm standard error of the mean (SEM).

\subsection{Results:}

\subsubsection{Timing of regional NO production in the rat asphyctic striatum and cerebellum:}

A bright fluorescent signal could be observed in the striatum and cerebellum of all pups after loading with DAF-2/DA. The fluorescent signal was localized intracellular in neuron-like cells and their proximal dendrites and in addition in vascular structures. Fluorescence in DAF-2 loaded slices of control rat pups was clearly visible throughout the entire striatum, predominantly in the dorso-medial part of the striatum. Within the cerebellum, DAF-2/T fluorescence was visible throughout the molecular and granular cell layer (Fig. 6.1). The cerebellum had higher $\mathrm{NO} /$ peroxynitrite levels than the striatum at all time-points after birth. In control rat pups, the intensity of the DAF-2/T fluorescent signal in the striatum was higher at P5 and P8 than at P12 (see Table 6.1). In the PA group, the intensity of the fluorescent signal was greater than in the control group at P5 and P8, but not at P12 (see Table 6.1). There was no apparent difference in fluorescence pattern or their intensity in the cerebellum between control and PA rats at P5, P8 or P12 (fig. 6.2). The fluorescent signal was however less intense at P12 than at P5 or P8.

Pre-incubation with the NO donor SNP led to a major increase in the intensity and density of the DAF-2/T fluorescent signal in the both striatum and cerebellum. Background levels were more intense after pre-incubation with SNP and almost all cells showed an extremely bright diffuse fluorescence (see Fig. 6.3 and Table 6.1). Pre-incubation with $N^{G}$-L-nitro-arginine $(0.1 \mathrm{mM}$, a NOS inhibitor) slightly suppressed the DAF-2/T fluorescent signal at P5, P8 and P12 in both striatum and cerebellum of PA rats at $35.5^{\circ} \mathrm{C}$. Lowering the incubation temperature to $25.5^{\circ} \mathrm{C}$ drastically lowered the signal.

Data on NO-mediated cGMP synthesis were obtained from slices incubated 
Table 6.1: Summary of the experiments performed with rat striatal slices at postnatal day (P)5, P8 and P12. Numbers represent the number of experiments. Per experiment, at least 3 slices throughout the striatum $(300 \mu \mathrm{m})$ were studied

\begin{tabular}{llllllll}
\hline & & Control & & \multicolumn{3}{c}{ Asphyxia } \\
\hline Age & & Basal & NG-L- Nitro-arginine & SNP & Basal & NG-L- Nitro-arginine & SNP \\
P5 & absence DAF & 0 & 1 & 0 & 0 & 2 & 0 \\
& weak DAF & 4 & 4 & 0 & 0 & 4 & 0 \\
& strong DAF & 4 & 0 & 5 & 6 & 1 & 3 \\
& very strong DAF & 0 & 0 & 2 & 2 & 0 & 4 \\
P8 & absence DAF & 0 & 2 & 0 & 0 & 1 & 0 \\
& weak DAF & 4 & 4 & 1 & 1 & 3 & 0 \\
& strong DAF & 3 & 0 & 3 & 4 & 3 & 6 \\
P12 & very strong DAF & 0 & 0 & 2 & 3 & 0 & 0 \\
& absence DAF & 1 & 3 & 0 & 0 & 5 & 0 \\
& weak DAF & 5 & 2 & 0 & 6 & 1 & 1 \\
& strong DAF & 0 & 0 & 6 & 1 & 0 & 5 \\
\hline
\end{tabular}

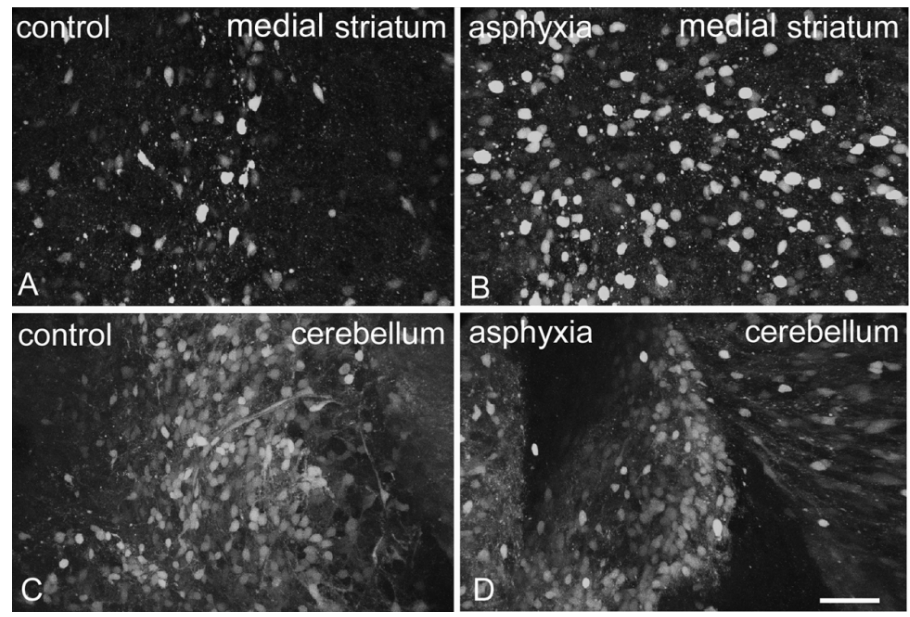

Figure 6.2: DAF-2 fluorescence in tissue slices from medial striatum (A, B) and cerebellum (C,D) of a control (A, C) and asphyctic rat (B, D) at postnatal day 8 . Images were taken throughout the slice $(300 \mu \mathrm{m})$ with a confocal laser scanning microscope, and combined into one image per area using an image analysis system. Scale bar is $50 \mu \mathrm{m}$ for all photographs. 

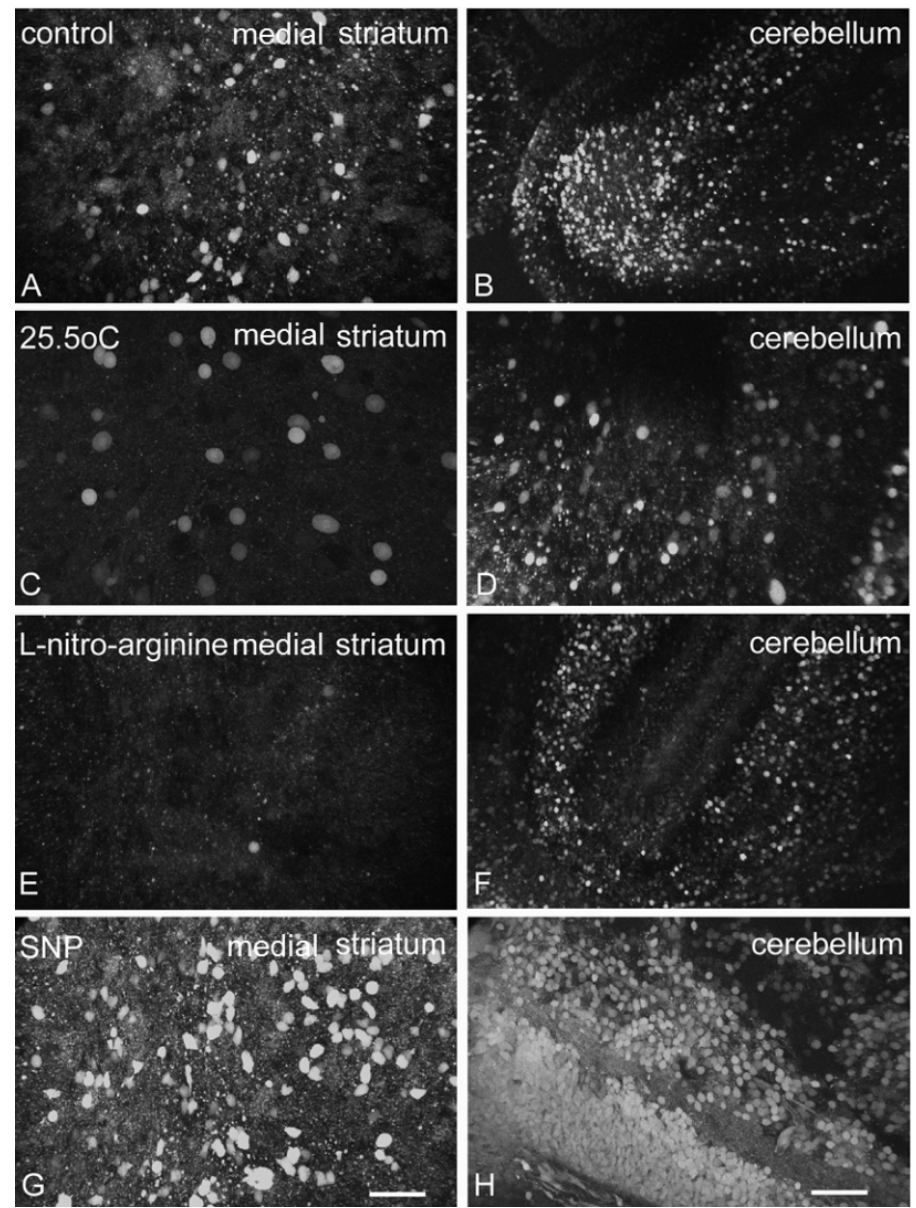

Figure 6.3: DAF-2 fluorescence in tissue slices of the medial striatum (A) and cerebellum (B) of a control rat incubated in the presence of $1 \mathrm{mM}$ IBMX at $35.5^{\circ} \mathrm{C}$ for $45 \mathrm{~min}$; and $(\mathrm{C}, \mathrm{D})$ in the presence of $1 \mathrm{mM}$ IBMX at $25.5^{\circ} \mathrm{C}$ for $45 \mathrm{~min} ;(\mathrm{E}, \mathrm{F})$ incubated in the presence of $0.1 \mathrm{mM} N^{G}$-nitro-L-arginine at $35.5^{\circ} \mathrm{C}$ for $45 \mathrm{~min}$; and $(\mathrm{G}, \mathrm{H})$ incubated in the presence of $0.1 \mathrm{mM} \mathrm{SNP}$ at $35.5^{\circ} \mathrm{C}$ for $45 \mathrm{~min}$. Hypothermia or incubation with $N^{G}$-nitro-L-arginine led to a decrease in fluorescent signal in both the striatum $(\mathrm{C}, \mathrm{E})$ and cerebellum $(\mathrm{D}, \mathrm{F})$. Pre-incubation with SNP led to a strong increase of the fluorescent signal in both the striatum $(\mathrm{G})$ and cerebellum $(\mathrm{H})$. Scale bar is $50 \mu \mathrm{m}$ for all photographs. 
with $1 \mathrm{mM}$ IBMX to inhibit PDE activity. Incubation of the rat slices at P5, P8 and P12 with $1 \mathrm{mM}$ IBMX resulted in intense cGMP immunoreactivity (cGMP-IR) in cell bodies and fibres in the striatum and cerebellum. Pre-incubation with $0.1 \mathrm{mM}$ NG-L-nitro-arginine abolished the cGMP signal, indicating that this NOS inhibitor abolished NOS activity and as a consequence NO production and cGMP synthesis. Incubation of the slices with SNP (0.1 $\mathrm{mM}$, a NO donor) resulted in an increase of cGMP-IR in cells and fibres in both the striatum and cerebellum of both groups. The PA group had increased cGMP-IR in cells and fibres throughout the striatum compared to the control group at $\mathrm{P} 5$ and $\mathrm{P} 8(P<0.05)$, but not at P12 $(P>0.05)$. nNOS-IR was observed in cell bodies and varicose fibres in grey and white matter of the striatum and cerebellum. Co-localization between cGMP and parvalbumin, but not between nNOS and parvalbumin, was found in cells and fibres in the striatum and cerebellum at P5, P8 and P12 (see Fig. 6.4). There was no detectable difference in nNOS-IR between control and asphyctic pups in the striatum or cerebellum. The results of the present study show that PA markedly increases $\mathrm{NO}$ /peroxynitrite during first postnatal week (P5 and P8, but not at P12), in the rat striatum. NO production in the cerebellum showed a similar trend, but no significant differences were observed between control and PA groups.

\subsubsection{Caspase-3 distribution and activity and astrogliosis in the rat asphyctic stratum and cerebellum:}

Caspase- 3 positive cell profiles were visible in both the grey and white matter of striatum and cerebellum of control and asphyctic pups during the first week of postnatal life. In the cerebellum, caspase- 3-positive cells were found within white matter and in the granular cell layer at P2 and P5. At P8, caspase- 3 positive cells were mainly observed within the granular cell layer. The caspase-3 immunohistochemistry was similar between the control and PA groups.

To assess the time course of caspase-3-like activity during normal development and after PA, we measured DEVD-cleavage in homogenates of cerebellum of control and PA rats during the first 15 days of postnatal life. The results of DEVD-cleavage activity are shown in fig. 6.5. The pattern of DEVD cleavage in the cerebellum was stable during the first week of life, followed by a decrease at $\mathrm{P} 11$ (P8 vs. $\mathrm{P} 11 ; P<0.01$ ) and an increase at P15. The time course of DEVD cleavage activity was not different between control and asphyctic group and cerebellum, but the DEVD cleavage was moderately higher in the PA group at P8 and P11 (PA vs. control; P < 0.05) and lower at P15 (not 


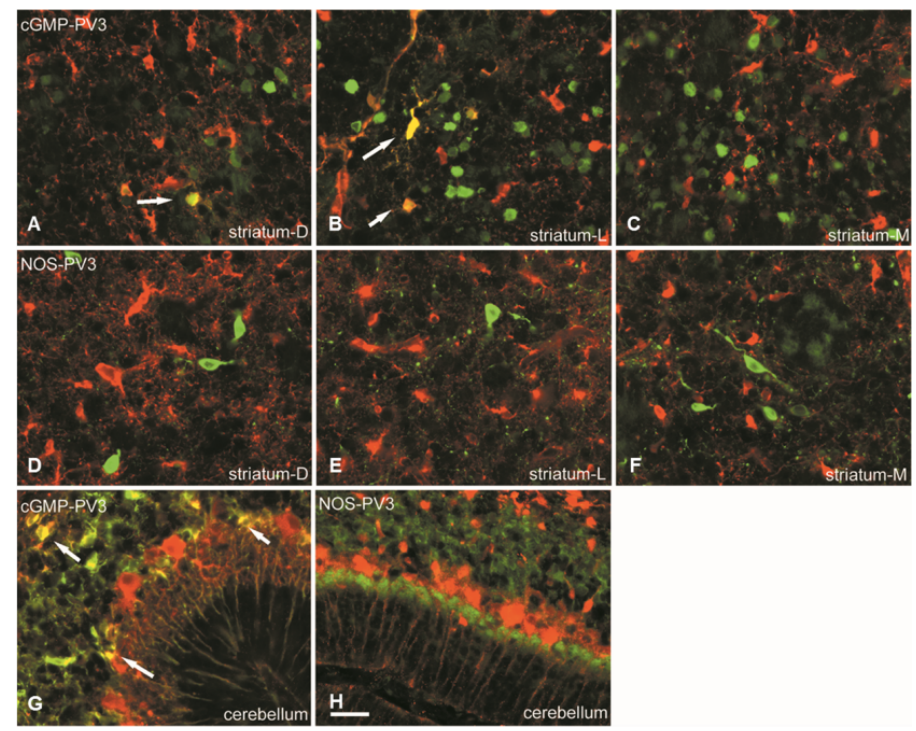

Figure 6.4: Double-labelling of parvalbumin (PV-3, in green) and NO-production markers, cGMP or nNOS (in red). Yellow cells indicate double-labelling and thus co-localization. Double labelling of PV-3 and cGMP in the dorsal (A), lateral (B) and medial striatum $(\mathrm{C})$ and cerebellum $(\mathrm{G})$. Double labelling of PV-3 and nNOS in the dorsal $(\mathrm{E})$, lateral $(\mathrm{F})$ and medial $(\mathrm{G})$ striatum and cerebellum $(\mathrm{H})$. Scale bar is 50 $\mu \mathrm{m}$. 


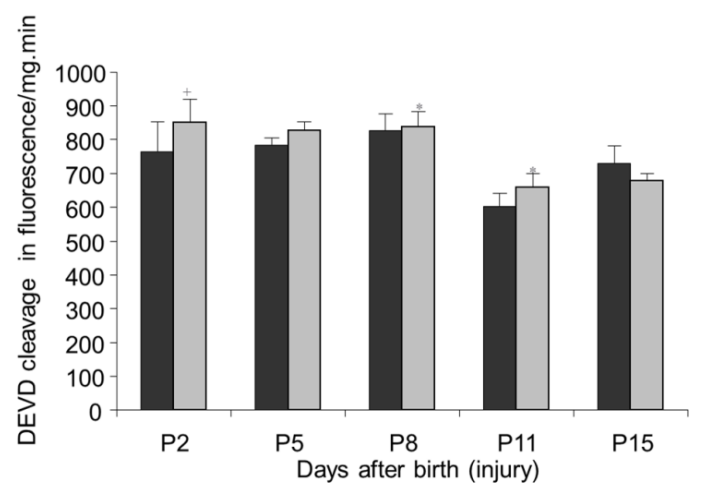

Figure 6.5: Caspase-3-like activity (DEVD-cleavage, expressed in cleaved AMC fluorescence per mg wet weight per minute) within the cerebellum of control (in black) and asphyctic (in grey) rats during the first 15 days after birth. The caspase-3-like activity after global asphyxia was compared using a two-way ANOVA with a Bonferroni correction for repeated measures. $+P=0.06{ }^{*} P<0.05$.

significant). This corresponds to the cerebellar DAF-2/T fluorescence which also did not show large variation between groups and which was higher at $\mathrm{P} 5$ and $\mathrm{P} 8$ than at later time-points. Overall, the regional distribution and timing of apoptosis in the striatum and cerebellum was similar between the PA and control groups in the first week, although there were modest relative increases in caspase-3 cleavage in the PA group up until P11. Astrocytes were not notably increased by PA (figure 6.6).

\subsection{Discussion:}

\subsubsection{Regional differences in NO-production and apoptosis:}

In the striatum, we observed a supra-physiological increase in NO/peroxynitrite and cGMP, which was not present in the cerebellum. The striatal NO production was not accompanied by an increase in nNOS expression. This is consistent with observations that iNOS, rather than nNOS or eNOS, is upregulated after asphyxia. iNOS greatly increases NO concentrations, which leads to peroxynitrite formation (Ikeno, Nagata et al. 2000). Another group showed decreased striatal nNOS-positive cells in organotypic cell cultures made 3 days after PA, accompanied by PA-related increases in nNOS-expressing cells in the substantia nigra, a region that normally has lower nNOS activity than the striatum (Klawitter, Morales et al. 2006, Klawitter, Morales et al. 2007). In 


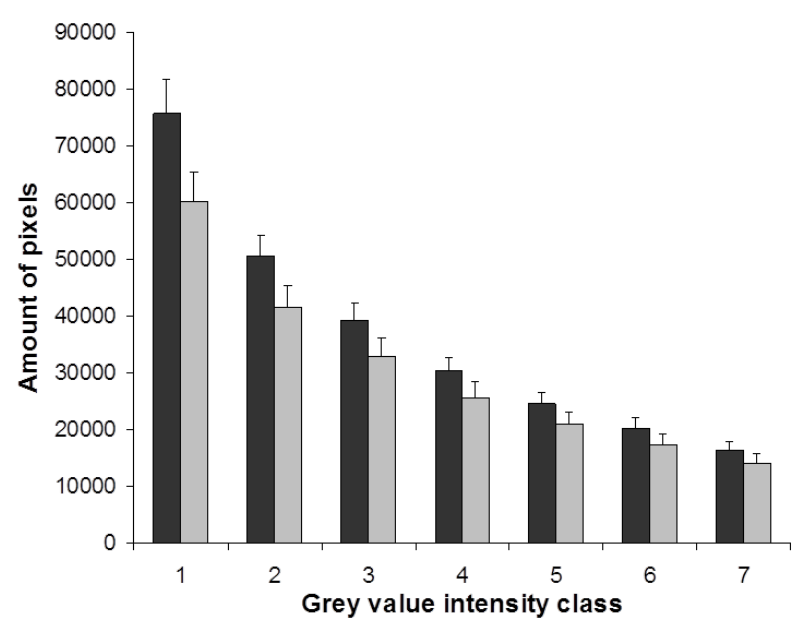

Figure 6.6: Histogram of division of pixels over intensity classes of GFAP immunoreactivity in striata of control (shown in black, $\mathrm{n}=5$ ) and asphyctic rats (shown in grey, $\mathrm{n}=5$ ), at P12. Immunofluorescence intensity was converted to grey values divided over 16 classes. The results of classes 10-16 are shown as almost no pixels were present in other classes. There was no difference in distribution or amount of pixels per grey value intensity class between the groups. Data are expressed as mean \pm SEM.

the cerebellum, the coupling between increased neuronal activity and local blood flow relies almost exclusively on NO (Rancillac, Rossier et al. 2006). Thus under physiological conditions, the cerebellum has the highest NOS activity and the highest concentration of glutamate and aspartate in the brain (Blanco, Molina et al. 2010). NO/peroxynitrite production in the striatum and cerebellum was higher on $\mathrm{P} 5$ and $\mathrm{P} 8$ than on $\mathrm{P} 12$. The effect of PA on NO was thus maximal during first postnatal week. The NO/peroxynitrite production was higher in the cerebellum than in the striatum at all time-points, but PA did not cause a supra-physiologic increase on top of the NO production seen in the control groups.

In both the striatum and cerebellum, there are sub-populations of neurons that would be vulnerable to supraphysiologic NO production - and relatively resistant neurons that express nNOS. Ninety-five percent of the striatal neurons are GABAergic projection neurons (medium spiny neurons) and the remaining $5 \%$ is made up by interneurons. The interneurons are divided into tonically active cholinergic neurons, fast-spiking GABAergic interneurons, which express parvalbumin, and low-threshold spiking interneurons, which express somatostatin, neuropeptide $\mathrm{Y}$ and nNOS. The low-threshold spiking interneurons are the primary source of striatal nNOS (Liljeholm and O'Doherty 2012, Lenz and 
Lobo 2013). nNOS is maximally expressed in the striatal regions where immature NMDA receptors are expressed (Black, Bedolli et al. 1995). Upon excessive stimulation of the NMDA receptors during PA, peroxynitrite is increased in the region (Ferriero, Arcavi et al. 1990, McQuillen and Ferriero 2004). The NOproducing interneurons are resistant to PA and NMDA-mediated excitotoxicity. However, the nearby striatal projection neurons are vulnerable to increased peroxynitrite formation. This selective vulnerability of the striatal projection neurons may result from a bystander effect attributable to their proximity to the enriched population of nNOS-expressing interneurons neurons (McQuillen and Ferriero 2004, Ehrlich 2012, Titomanlio, Fernández-López et al. 2015). The relative resistance of the low-threshold spiking nNOS interneurons is echoed by our observations. The nNOS immunoreactivity did not change, and thus presumably PA did not alter the amount of nNOS-expressing interneurons in the striatum. However, NO/peroxynitrite and cGMP increased in the striatum and cGMP partially co-localized with parvalbumin neurons (figure 6.4), which indicates that there was more NO available to the parvalbuminergic neurons. Parvalbumin fast-spiking and cholinergic interneurons in the striatum have demonstrated vulnerability to PA (Kohlhauser, Mosgoeller et al. 1999, de Vente, Markerink-van Ittersum et al. 2000, Van de Berg, Schmitz et al. 2002).

In the cerebellum, nNOS is expressed by both the mature granule neurons and the molecular layer interneurons (basket and stellar cells) (Contestabile 2012) and these cells are thus relatively more resistant to NO/peroxynitrite toxicity. The Purkinje cell-population (which express parvalbumin, but not nNOS) is sensitive to peroxynitrite, but these cells also need a small amount of NO to survive (Oldreive, Gaynor et al. 2012). During normal postnatal development, the expression and activity of cerebellar nNOS increases slowly throughout the first week. The granule neurons only show detectable NOS reactivity after migrating to the internal granular layer (Contestabile 2012). We observed NO/peroxynitrite production throughout the molecular and granular layer of the cerebellum in both control and PA rats. nNOS expression and cGMP production occurred throughout the cerebellum in both groups at 35.5 ${ }^{\circ} \mathrm{C}$. Lowering the temperature by $10{ }^{\circ} \mathrm{C}$ drastically lowered NO production. This correlates with the clinical observations that selective head cooling is beneficial after PA (Guillet, Edwards et al. 2012).

We only observed changes in the cerebellar caspase-3-like activity in the second week of postnatal life in both control and asphyctic rats. The pattern of caspase-3-like activity in the cerebellum is unique in that it declines after the first week of postnatal life, but increases again afterwards (P15). There was a 
modest increase in caspase- 3 activity in the asphyctic groups up until P11, but overall the patterns of caspase-3-like activity (and distribution of caspase-3 immunoreactive cells) was similar between groups and probably reflects physiological cell death. Apoptotic cell death is crucial for the normal development of the CNS and occurs in all brain areas during foetal and postnatal life (Devoto, Bogetti et al. 2013).

Although the striatum as a whole is susceptible to PA, not all striatal neurons are affected equally after PA. The nNOS-expressing interneurons were spared in our model. This is also seen in chronic degenerative diseases of the striatum that lead difficulties in motor control, like Huntington's disease. In Huntington's, the striatal neurons that do not release NO degenerate during the disease course, but the nNOS-producing interneurons are spared. The striatal projection neurons that form cortico-striatal network are particularly sensitive to glutamate-induced excitotoxicity and NO release (Ehrlich 2012, Canzoniero, Granzotto et al. 2014).

Previous NO-related research in the same model of asphyxia showed that increased striatal cGMP production is still evident at P10 (Loidl, De Vente et al. 1998). However, directly after PA, when the fetal circulation and metabolism is still maintained, there were no apparent changes in the activity or transcription of nNOS (Lubec, Kozlov et al. 1999) or inducible NOS (Calamandrei, Venerosi et al. 2004). Early intervention in NO-production could have long-term therapeutic effects. At 6 months, PA-related increases in striatal NOS activity were still apparent. The NOS-containing neurons had ultra-structural changes including cytomegaly and the surrounding neurons showed increased degeneration (Capani, Loidl et al. 1997). In the same regions, neurons that express nicotinamide adenine dinucleotide phosphate diaphorase (NADPH-d) - a marker of NO synthesis - had similar cytomegalic morphology. The gross amount of NADPH-d expressing neurons was not altered by PA (Loidl, Capani et al. 1997). The results of the timing-data suggest that the optimal therapeutic window for a NO-targeted therapy in the preterm infant would be in the first week after PA. Overall, our results show that reducing NO production could be a potential therapy to reduce striatal damage. The protective effects of NO-reduction may be limited to the regions with supraphysiological increases in NO. 


\subsection{Conclusion:}

From these observations, we conclude that PA has a greater impact on $\mathrm{NO}$ /peroxynitrite production in the striatum than in the cerebellum. Although $\mathrm{NO}$ /peroxynitrite was observed throughout the cerebellum, this can be ascribed to the high nNOS activity of the region. The greatest increase in NOproduction occurred during the first week, although it was previously reported that this change is not evident directly after PA. A therapeutic window exists within the first week after PA, during which inhibitors of NO-production would likely have their best therapeutic effect in the preterm brain. Although this type of intervention would likely only show regional benefits in the brain-regions with lower initial NO-production, attenuating striatal neuron loss could have a motor-benefit after PA. Astrocytes were not notably increased by PA, thus the striatal NO/peroxynitrite increase is likely independent of infiltration by inflammatory cells. Targeting inflammation alone is likely to be ineffective after PA and alternate pathways should be considered.

\subsection{Acknowledgements:}

This research was partially supported by the Sistema de Investigación y Desarrollo (SINDE) of the Universidad Católica de Santiago de Guayaquil, Guayaquil, Ecuador, through the grant No SIU- 319: Perinatal asphyxia and stem cell treatment and the Education, Audiovisual and Culture Executive Agency, EU through the grant No 2013-3396. The generous gift of the antiserum against parvalbumin by Dr. Piers Emson is gratefully acknowledged. The authors are grateful to L. Veerbeek, M. Huiban-Minnaar, M.O. Lopez-Figueroa,M. Markerink and H.P.J. Steinbusch for their expert technical assistance and helpful suggestions. M. Barkhuizen is funded by the National Research Foundation of South Africa (Grant specific reference number 89230 and 98217). All views expressed in this article are those of the authors and not of the funding agencies. 



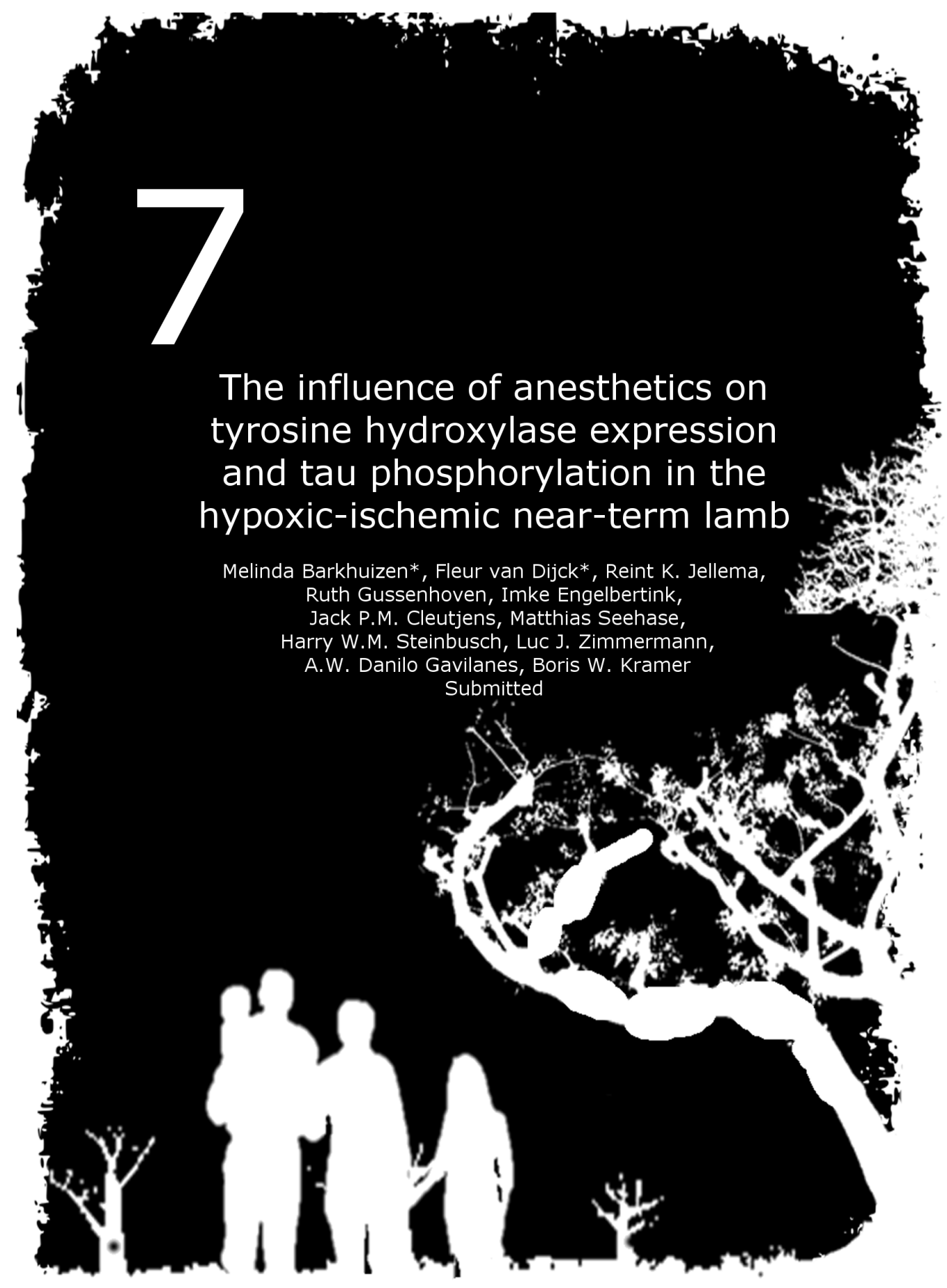




\title{
The influence of anesthetics on tyrosine hy- droxylase expression and tau phosphoryla- tion in the hypoxic-ischemic near-term lamb
}

Melinda Barkhuizen*, Fleur van Dijck*, Reint K Jellema, Ruth Gussenhoven, Imke Engelbertink, Jack P.M. Cleutjens, Matthias Seehase, Harry W.M. Steinbusch, Luc J. Zimmermann, A.W. Danilo Gavilanes, Boris W. Kramer

* These authors contributed equally

Submitted to Pediatric Research

\begin{abstract}
:
Background: The dopaminergic neurotransmitter system is especially vulnerable to excitotoxicity by excessive glutamate release after hypoxic ischemic encephalopathy (HIE). Several general anesthetics reduce glutamate neurotransmission, which could provide neuroprotection. However, these anesthetics may also increase tau phosphorylation, which is associated with adverse neurological outcomes.
\end{abstract}

Methods: We investigated whether two commonly used anesthetics propofol and isoflurane - can reduce the expression of the dopamine-synthesis enzyme, tyrosine hydroxylase (TH), in the substantia nigra (SN) with immunohistochemistry. We further investigated whether this altered microglial response and tau phosphorylation in the midbrain grey matter structures and hippocampal white matter of a near-term sheep model of HIE after umbilical cord occlusion (UCO).

Results: UCO reduced the number of dopaminergic neurons in the SN $(\mathrm{p}<0.05)$ at 8 hours after the insult but propofol anesthesia significantly prevented the neuron loss. This neuroprotective effect was not associated with reduced microglial proliferation in the SN. Both UCO and anesthetic use additively increased tau phosphorylation in the SN region, thalamus, and hippocampus, in comparison to gestational age controls.

Conclusion: Conclusion: The choice of anesthetic is an important consideration during an emergency C-section. Propofol anesthesia could potentially limit neuron loss within the substantia nigra, but it was associated with increased tau phosphorylation which could be pathological if sustained. 
Keywords: Brain injury; hypoxia-ischemia; preterm; neuroprotection; anesthesia; substantia nigra; tau 


\subsection{Introduction:}

Hypoxic-ischemic encephalopathy (HIE), remains an important medical challenge. Survivors of HIE have a high risk of life-long disabilities such as cerebral palsy, learning difficulties and attention deficit hyperactivity disorder (ADHD) (Volpe, 2009, Van Handel et al., 2007). Damage to the substantia nigra (SN) could partially explain the motor and behavioral deficits after HIE from early gestation to full term, as seen upon post-mortem examination of human infants from 32.5 weeks of gestation to term with prolonged HIE (Pagida et al., 2013). The SN indirectly controls movement through the nigrostriatal dopaminergic tract, which is frequently affected in rodent models of HIE representing human gestational ages varying from the extreme preterm brain at 28 weeks (Barkhuizen et al., 2017) to the full term brain (Ezquer et al., 2006). In humans, SN abnormalities have also been associated with ADHD (Romanos et al., 2010).

Neuron loss after HIE can occur in several phases. In the first phase of injury, the release of glutamate is increased, which results in an accumulation of excitatory amino-acids outside the cell. This is followed by the second phase of injury associated with by microglial response and neuronal deterioration at 6-15 hours in humans and at 5-48 hours after HIE in the sheep (Davidson et al., 2015, Perlman, 2006, Bennet et al., 2006).

HIE increases inflammatory cytokine production mediated by microglia, which worsens the neurological injury (Lai et al., 2017). Therapies which could prevent excessive glutamate release and microglial-mediated inflammation could be neuroprotective after HIE (Buonocore et al., 2012). Several general anesthetics could potentially reduce excitotoxicity by antagonizing glutamatergic $\alpha$-amino-3-hydroxy-5-methyl-4-isoxazolepropionic acid (AMPA) and N-Methyl-D-aspartate (NMDA) receptors to various degrees (Rudolph and Antkowiak, 2004). Whilst anesthetic exposure is thought to be toxic to the healthy developing brain through increased apoptosis and disrupted neurogenesis (Sinner et al., 2014), anesthetics may be neuroprotective in the context of an ischemic injury through reductions in apoptosis, activation of pro-survival pathways, reducing excitotoxicity and reducing inflammation (Archer et al., 2017). Anti-inflammatory effects vary between anesthetics, with studies suggesting that inhalation anesthetics enhance microglial cytokine release, whilst propofol suppresses cytokine release (Ye et al., 2013) by reducing microglial activation after brain injury (Luo et al., 2013). NMDA-receptor antagonism also has a direct anti-inflammatory effect (Jander et al., 2000). We have therefore developed a lamb model at $89 \%$ of gestation, in which anesthetic administration to the ewe prior to emergency C-section acts as an early treatment to 
the near-term fetus in distress due to umbilical cord occlusion (UCO), which results in an acute hypoxic-ischemic insult. The anesthetic administration is continued in the newborn lamb post-delivery as an early post-insult therapy (Seehase et al., 2013, Seehase et al., 2011, Seehase et al., 2015, Smit et al., 2013). We investigated the use of propofol and isoflurane in this scenario as they are both rapidly acting anesthetics that can be administered during an emergency C-section, and both anesthetics inhibit glutamatergic NMDA and AMPA-receptors (Rudolph and Antkowiak, 2004). We have previously shown several neuroprotective effects of propofol anesthesia in this model, including functional improvements of the auditory pathway (Smit et al., 2013) and molecular benefits such as reduced levels of pro-apoptotic caspases, increased levels of anti-apoptotic proteins, less reactive oxygen species, less mitochondrial damage and reduced $\gamma$-aminobutyric acid (GABA)- and NMDA-receptor expression in the brain (Seehase et al., 2015, Seehase et al., 2011, Smit et al., 2013).

Unfortunately, general anesthetics are also known to cause aberrant tau phosphorylation, which is thought to exacerbate the presentation of neurodegenerative diseases (Whittington et al., 2013). Soluble tau is a microtubule associated protein, which modulates the stability of axonal microtubules to allow the formation of axons and dendrites during normal neurological development. Translocation of the tau from the axons and post-translational modifications, like hyperphosphorylation disrupt tau's microtubule stabilizing functions and are associated with neuropathology (Yu et al., 2009, Spillantini and Goedert, 2013). After hypoxic or traumatic injuries, neuronal tau is released extracellularly and can function as a general biomarker of axonal injury. Elevated serum or cerebrospinal fluid tau levels have been linked to worse outcomes after neonatal encephalopathies such as HIE (Takahashi et al., 2014, Wu et al., 2017), bilirubin-induced neurotoxicity (Okumus et al., 2008) and infantile spasms due to West Syndrome (Inoue et al., 2012). The primary aim of this study was to determine whether propofol and isoflurane could maintain dopaminergic neurotransmission by preventing a reduction in tyrosine-hydroxylase expressing neurons in the substantia nigra after UCO, since this could potentially reduce long-term motor morbidity and attention deficits (Barkhuizen et al., 2017, Strackx et al., 2008). The secondary objectives of this study were to determine the effect of anesthesia therapy on microglial response and tau-hyperphosphorylation after UCO since these factors are known to contribute to SN neuronal loss (Irwin et al., 2013, Heneka et al., 2014). The final objective of this study was to determine whether changes in microglial response and tau phosphorylation were limited to the substantia nigra, or widespread throughout susceptible regions. For this, we extended our 
investigation of microglia and tau to other regions known to be vulnerable to hypoxic-ischemia around term, such as the hippocampus, fornix and thalamus (Volpe, 2012, Counsell et al., 2010).

\subsection{Materials and methods:}

\subsubsection{Study design:}

The study was approved by the Animal Ethics Research Committee, Maastricht University, The Netherlands. Care was taken to minimize the number of sheep used and to minimize the discomfort of the sheep in accordance with the EU Directive 2010/63/EU for animal experiments. The study design was described in detail previously (Seehase, Jellema et al. 2011). In brief, date-mated Texel ewes and their 23 singleton late-preterm fetuses of both sexes at a mean gestational age of $133-134$ days ( $89 \%$ of gestation) were used in the experimental protocol. The ewes were randomized for the allocation of sedation with either propofol or isoflurane, which was continued in the preterm lamb after birth. This made the blinded administration of the drug prohibitive. The fetuses were randomized for the induction of global hypoxia-ischemia by umbilical cord occlusion (UCO) or a sham-procedure. Twelve sheep fetuses were subjected to total UCO in utero, and 11 fetal sheep served as sham controls. In each group, about half of the pregnant ewes and their offspring were sedated with propofol and the other ones with isoflurane. The final group sizes were: 6 UCO-isoflurane (UCO-I); 6 UCO-propofol (UCO-P), 5 Sham isoflurane (Sh-I); 6 Sham propofol (Sh-P). An additional four lambs were euthanized directly after preterm delivery and served as gestational age (GA) controls. The birth weights and times of UCO was comparable between groups (see Seehase, Jennekens et al. (2015)).

\subsubsection{Induction of UCO and sedation:}

The experimental protocol used in this study has been published previously (Seehase, Jellema et al. 2011, Seehase, Houthuizen et al. 2013, Seehase, Jennekens et al. 2015). Briefly, the pregnant ewes were intubated and randomized to receive general anesthesia maintained with isoflurane (1\% to $2 \%$ ) or propofol $(25 \mathrm{mg} / \mathrm{kg} / \mathrm{h}$ ) during the C-section. Both anesthesia types were supplemented by continuous remifentanil infusion $(3 \mu \mathrm{g} / \mathrm{kg} / \mathrm{min})$. The fetus was born through a small incision in the uterus, instrumented with an endotracheal tube and catheters in the femoral artery, umbilical artery and external jugular vein. The umbilical cord was gently extracted through the incision of the uterus, 
and a vascular occluder (OC16HD, $16 \mathrm{~mm}$, IN VIVO METRIC, Healdsburg, California, USA) was placed around the umbilical cord. The umbilical cord was occluded until the mean arterial blood pressure (MABP) dropped below $30 \mathrm{~mm} \mathrm{Hg}$. From that time point onwards, the occlusion was continued for exactly 2 min. The total duration of umbilical cord occlusion (UCO) required was $10.7 \pm 0.3$ minutes in the propofol-treated lambs and $11.3 \pm 0.6$ minutes in the isoflurane-treated lambs ( $\mathrm{p}=$ not significant) (Seehase et al., 2013, Seehase et al., 2015). After the end of the occlusion, the lambs were taken out and they were given 20 seconds during the C-section while experiencing tactile stimulation to recover spontaneously. All the UCO animals developed bradycardia (heart rate $<30 / \mathrm{min}$ ), and all but one UCO-propofol lamb needed resuscitation. The lambs were initially ventilated with a resuscitator bag and resuscitated with a heart massage during transport an incubator (IW930 Series CosyCot $^{\mathrm{TM}}$ Infant Warmer, Fisher \& Paykel Healthcare), where the lamb was connected to a ventilator Servo 900C (Siemens-Elema, Solna, Sweden) and put into plastic foil to prevent cooling effects. The incubator constantly maintained the lamb's core temperature of $38-38.5^{\circ} \mathrm{C}$, as controlled with a rectal thermometer. Chest compression was continued until the heart rate was $>80 / \mathrm{min}$. The time needed to achieve this heart rate spontaneously was 9.0 $\pm 0.3 \mathrm{~min}$ in the propofol-treated lambs and $9.0 \pm 0.4 \mathrm{~min}$ in the isofluranetreated ones. Adrenaline was administered in augmenting dosage from 30 over $60 \mu \mathrm{g}$ to $100 \mu \mathrm{g}$. A volume bolus of ringer lactate of $10 \mathrm{~mL} / \mathrm{kg}$ body weight was administered after the first dose of adrenaline. The sedation was maintained either with isoflurane ( $0.5 \%$ to $1.0 \%$ ) or propofol (1 to $3 \mathrm{mg} / \mathrm{kg} / \mathrm{h})$, according to the maternal treatment and supplemented with remifentanil $(3 \mu \mathrm{g} / \mathrm{kg} / \mathrm{min})$ in both groups. The ventilation and sedation were continued for $8 \mathrm{~h}$ after delivery. The sham-treated fetuses had the same instrumentation and sedation as the UCO-groups, without occlusion of the umbilical cord (Seehase et al. 2013, Seehase et al., 2015). Initial pH values before birth were similar in all the groups $(7.26 \pm 0.01)$. After UCO followed by successful resuscitation, the $\mathrm{pH}$ decreased to $6.91 \pm 0.03$ in the UCO propofol group, whilst the $\mathrm{pH}$ in the sham-operated propofol group was $7.10 \pm 0.03$. In the UCO isoflurane group, the $\mathrm{pH}$ was $6.84 \pm 0.01$ after resuscitation as compared with $7.07 \pm 0.02$ in the sham-operated isoflurane group $(\mathrm{p}<0.05)$. At $8 \mathrm{~h}$ after delivery, the lambs were euthanized using an intravenous injection of T61 (Veterinaria AG) (Seehase et al., 2013, Seehase et al., 2015). These experiments modelled an acute severe asphyxic insult leading to a cardiac arrest and clinical convulsions in all lambs that underwent UCO, despite the sedation. The EEG indicated also ongoing seizure activity in the UCO groups. The combination of cardiac arrest, in all but one UCO lamb, and seizure activity is consistent with the clinical picture 
of a severe asphyxic insult (Seehase et al., 2013, Seehase et al., 2015). The birth weights and general characterization of the lambs used in this study have been published previously (Seehase et al., 2015, Seehase et al., 2013, Seehase et al., 2011, Smit et al., 2013).

\subsubsection{Immunohistochemistry:}

The fetal brain was removed from the skull. The right hemisphere was submersion fixated in ice-cold $4 \%$ paraformaldehyde for 3 months, after which it was stored in phosphate buffered saline with sodium azide until imbedding in gelatin and placed $4 \%$ paraformaldehyde for an additional 2 hours. Serial coronal sections $(50 \mu \mathrm{m})$ were cut on a Leica VT $1200 \mathrm{~S}$ vibrating microtome (Leica Biosystems, Nussloch, Germany). Free floating sections at the level of mid-thalamus and posterior hippocampus to the substantia nigra were used in the immunohistochemical analyses. To detect and quantify dopaminergic neurons we conducted anti-tyrosine hydroxylase $(\mathrm{TH})$ immunohistochemistry on every $10^{t h}$ slide. Antigen retrieval was performed on free-floating sections with incubation in $0.1 \mathrm{M}$ citrate buffer at $60^{\circ} \mathrm{C}$ for 20 minutes followed incubation with a mouse-monoclonal anti-tyrosine hydroxylase antibody (1:800, Immunostar, Hudson, WI, USA, cat. ID. 22941) for 3 days. The slides were then incubated with donkey anti-mouse-biotin (1:400; Jackson Immunoresearch, Suffolk, UK) followed by incubation with the Avidin-Biotin-Complex (ABC, 1:800 Vector, Peterborough, UK). Staining was visualized with 3,3/-diaminobenzidine solution (DAB). Microglia was detected in an adjacent series of every tenth section by immunohistochemistry against anti-ionized calcium-binding adapter molecule 1 (IBA1). Slices were incubated overnight with a monoclonal rabbitanti-IBA1 primary antibody (1:1000, WAKO chemicals, Nuss, Germany, cat. 019-19741) followed by incubation with donkey anti-rabbit-biotin (1:800; Jackson Immunoresearch, Suffolk, UK), ABC (1:800; Vector, Peterborough, UK). Staining was visualized with DAB using nickel intensification (0.2\%) (DAB-Ni). Tau phosphorylation was detected in additional series of every $10^{t h}$ section. The sections were pretreated in $98 \%$ formic acid for 20 minutes for antigen retrieval and incubated with mouse-monoclonocal primary antibodies against various stages of tau phosphorylation seen in the tauopathies. We mea,ured early tau phosphorylation with an anti- phosphorylated Ser-202 tau antibody (CP13, 1:100, generous gift from Dr. Peter Davies, Albert Einstein College of Medicine, USA), and late-stage tau phosphorylation with an antibody against phosphorylated Ser396/Ser404-tau (PHF1, 1:100, Dr. Peter Davies). We also used an antibody against early conformational changes in tau (MC1, 1:100, Dr. Peter Davies). This was followed by incubation with donkey anti-mouse-biotin (1:400; Jackson Immunoresearch, Suffolk, UK) followed by ABC (1:800; Vector, 
Peterborough, UK) and DAB visualization. Stained sections were mounted on glass slides, embedded using Depex (Merck, Amsterdam-Zuid, Netherlands) and cover slipped. The brain tissue from a transgenic Alzheimer disease mouse (5XFAD, The Jackson Laboratory, Bar Harbor, USA) and post-mortem hippocampal slices from an Alzheimer's disease patient (a generous gift of Fred van Leeuwen) were used as a positive control.

\subsubsection{Quantitative and qualitative analysis:}

All the analyses were conducted by a blinded investigator. For the analysis of IBA-1 and tau immunoreactivity, digital images of the hippocampal CA3 and DG layers (tau) and white-matter layers adjacent to these layers (IBA-1), fornix, thalamus, midbrain region surrounding the $\mathrm{SN}$ were taken at a $2 \mathrm{x}, 10 \mathrm{x}$ and 20x magnification using an Olympus DP 70 digital camera attached to an Olympus AX 70 microscope and Cell-P software Version 2.3 (Soft Imaging System, Münster, Germany). The position of the SN was determined by overlaying the TH-positive slides. The 20x magnification images were used for the IBA1 analysis. The area fraction of IBA-1 immuno-reactivity was determined with a standard intensity threshold to determine positive staining using ImageJ 1.5 software. For the tau phosphorylation, images taken at 10x magnification were scored according to coloring intensity ranging from 1-4.

For the TH-analysis, photos of the SN were taken at $5 \mathrm{x}$ magnification with a Leica DM3000 microscope and the TH-positive cells were counted using the Leica QWin software (Leica Microsystems, Cambridge, UK) and expressed as total cell count over 4 slides in the $\mathrm{SN}$ and as cells $/ \mathrm{mm}^{2}$. The SN was defined as a stripe originating upwards from the VTA. The SN and VTA were separated by a thin line lacking cells. The cumulative neuron count over 4 slides was used in the calculations. At least 4 slides per lamb on the same Bregma level were used for all the analyses.

\subsubsection{Data analysis:}

Statistical analysis was performed with the Stata10 software package (Statacorp, TX, USA). All the data were assessed with the non-parametric KruskalWallis test with post-hoc Dunn's analysis. The Bonferroni correction for multiple testing was applied to the microglia and phospho-tau immunohistochemistry where multiple regions were investigated. Statistical significance was defined as two-tailed p-values $<0.05$. The results are shown as average \pm S.E.M. Graphs were made with GraphPad Prism 6 (GraphPad, La Jolla, CA, 


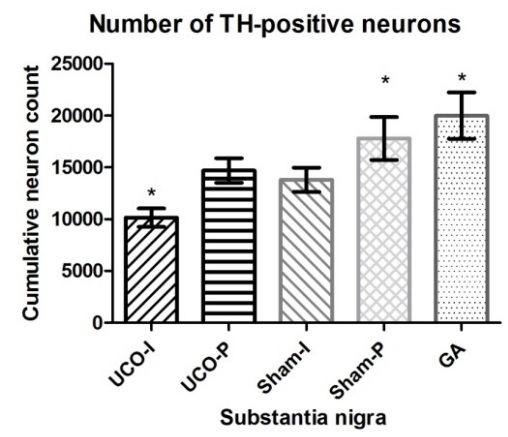

Figure 7.1: The cumulative TH-neuron counts in the SN over 4 slides. The graph shows the mean \pm S.E.M. of the counts. The UCO-isoflurane group had a significantly lower number of neurons in comparison to the sham-propofol and GA control groups (indicated with an $*$ ).

USA).

\subsection{Results:}

\subsubsection{TH-positive neurons in the substantia nigra:}

The results of the TH-counts at 5x magnification are shown in figure 7.1 and the corresponding immunohistochemistry photos are shown in figure 7.2. The TH-neurons were still migrating at the time of sampling and differences in the shape of the $\mathrm{SN}$ were observed between the lambs. In some lambs, the TH-neurons have already nearly-completed migration into the characteristic line-shape of the SN, whilst in others, TH-immunoreactivity was found throughout the midbrain (number of lambs with incomplete migration: 1/6 UCO-I, 3/6 UCO-P, 2/5 Sh-I, 3/6 Sh-P, 1/4 GA). For the purposes of our cell counting, we only counted TH-neurons which fell within a line-shape on four serial slides, on corresponding Bregma levels present in all sheep. Overall, UCO reduced the cumulative number of neurons counted in the SN (UCO-I 10150 \pm 47 neurons; UCO-P 14696 \pm 51 neurons; Sh-I 13806 \pm 51 neurons, Sh-P $17790 \pm 71$ neurons, GA $19995 \pm 67$ neurons) (UCO vs. Sh $\mathrm{p}<0.05$, UCO-I vs. Sh-P/GA $\mathrm{p}<0.01$, UCO-I vs. Sh-I and UCO-P vs. Sh-P/GA not significant). Propofol mitigated some of the neuron loss due to UCO. The density of neurons did not vary significantly between groups (UCO-I $72.35 \pm 4.23$ neurons $/ \mathrm{mm}^{2}$, UCO-P $77.96 \pm 5.69$ neurons $/ \mathrm{mm}^{2}$, Sh-I $76.12 \pm 3.98$ neurons $/ \mathrm{mm}^{2}$, Sh-P $74.55 \pm 4.19$ neurons $/ \mathrm{mm}^{2}$, GA $113.99 \pm 3.59$ neurons $/ \mathrm{mm}^{2}$ ). 

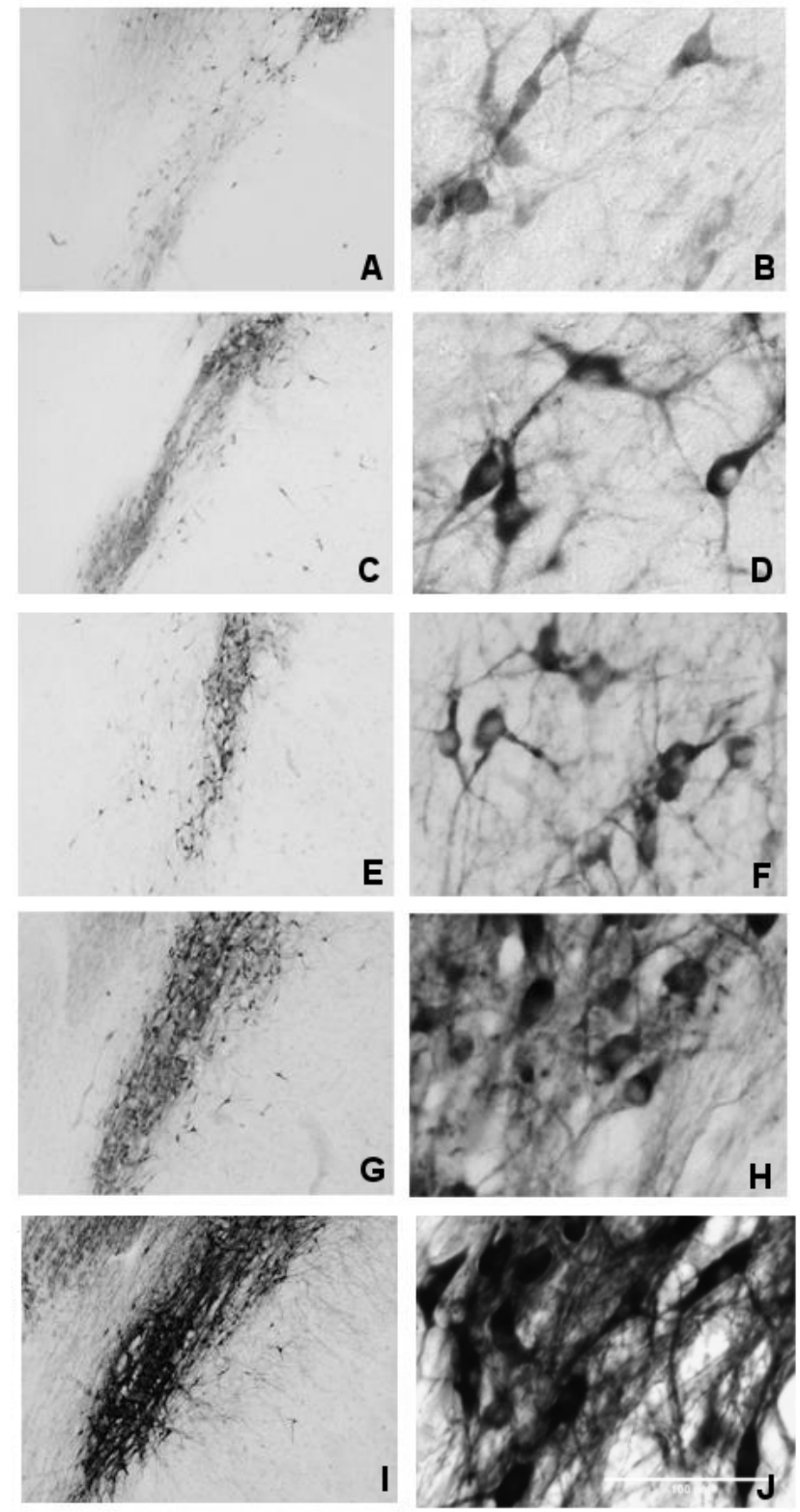

Figure 7.2: Representative photos of the TH-staining in the $\mathrm{SN}$ at $4 \mathrm{x}$ and $40 \mathrm{x}$ magnification. The scale bar represents $100 \mu \mathrm{m}$. Key: A-B: UCO-I, C-D: UCO-P, E-F: SH-I, G-H: SH-P, I-J: GA 


\subsubsection{Microglial proliferation:}

The percentage area covered by IBA-1 positive microglia was assessed in the grey matter in the midbrain area surrounding the substantia nigra (SN) and thalamus, and in the white matter of the fornix, stratum moleculare below the dentate gyrus (S.M. of the D.G.) and stratum oriens of the CA3 layer (S.O. of the CA3) in the hippocampus. The SN was defined by comparison with the TH-immunohistochemistry on adjacent slides. The IBA-1 proliferation and tau phosphorylation was more pronounced in the areas adjacent to the SN than in the SN- itself (figure 7.3). In the SN region, there was a trend towards less microglial proliferation in the UCO groups which did not reach statistical significance (UCO-I 27.75 $\pm 4.17 \%$, UCO-P $28.24 \pm 4.37 \%$, SH-I $33.86 \pm 4.35 \%$, SH-P $34.92 \pm 4.64 \%$, GA $34.59 \pm 4.51 \%, 0.05<\mathrm{p}<0.1$ ). The percentage IBA-1 proliferation also did not reach statistical significance in the fornix or the S.O. layer in the CA3 region of the hippocampus.

In the thalamus, the UCO-P group had significantly lower microglial proliferation $(12.14 \pm 3.14 \%)$ than the non-UCO exposed groups (SH-I $17.54 \pm 3.39 \%$, SH-P $16.94 \pm 3.75 \%$, GA $14.71 \pm 2.82 \% ; \mathrm{p}<0.05-\mathrm{p}<0.001)$ and the UCO-I group $(15.70 \pm 3.59 \%, \mathrm{p}=$ n.s. $)$. In the S.M. layer, microglial proliferation was higher in the non-UCO exposed groups (SH-I 33.09 \pm 3.71 , SH-P $40.71 \pm 4.08 \%$, GA $26.95 \pm 3.06 \%)$. The SH-P group had significantly more microglial proliferation than the UCO exposed groups (UCO-I, $30.46 \pm 3.71 \%$, UCO-P $28.36 \pm 3.65 \%$; SH-P vs. UCO-I/UCO-P $\mathrm{p}<0.001)$. The microglial proliferation of the thalamus and S.M. is shown in figure 7.4.

\subsubsection{Tau phosphorylation}

The CP-13 antibody, against phosphorylated Ser-202 tau, showed regionspecific effects in areas assessed. In the $\mathrm{SN}$, direct comparison with the TH-staining showed that there was more immunoreactivity in the area adjacent to the SN than in the dopaminergic neurons themselves (figure 3). The intensity of the CP-13 staining was overall higher in both UCO-plus-anesthesia groups (UCO-I: $2.12 \pm 0.09$, UCO-P: $2.22 \pm 0.14$ ) than in the corresponding shamanesthesia groups (SH-I: $1.80 \pm 0.14$, SH-P: $1.97 \pm 0.16$ ), which was higher than the absolute control group (GA 1.31 \pm 0.15 , GA vs. UCO-I/UCO-P p $<0.001$, GA vs. SH-P $\mathrm{p}<0.05)$.

In the thalamus, a similar effect was seen with a pronounced increase in the UCO-P group $(2.53 \pm 0.09)$ in comparison to the other anesthesia-exposed groups (UCO-I: $2.12 \pm 0.17$, SH-I: $2.22 \pm 0.12$, SH-P: $2.01 \pm 0.09$, UCO-P vs. 

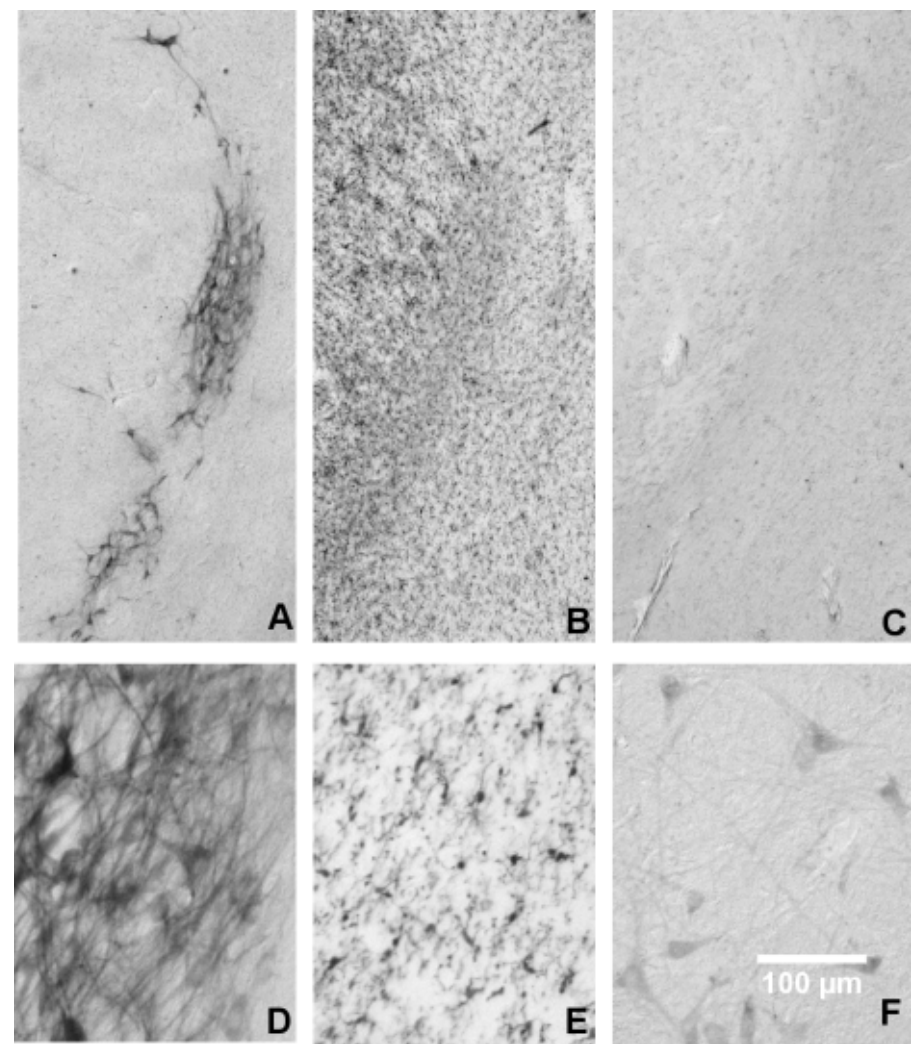

Figure 7.3: A comparison of the TH (A,D), IBA-1 (B,E) and CP-13 (C,F) immunohistochemistry in the substantia nigra of an UCO-I lamb at $2 \mathrm{x}$ and $10 \mathrm{x}$ magnification. In the IBA-1 (microglia) and the CP-13 (phosphorylated ser-202 tau) images the dopaminergic neurons of the substantia nigra (with the TH immunohistochemistry) is visible as an area with a lower reactivity than the surrounding midbrain areas. There was no significant difference in IBA-1 reactivity between groups in the substantia nigra region. The scale bar represents $100 \mu \mathrm{m}$. 


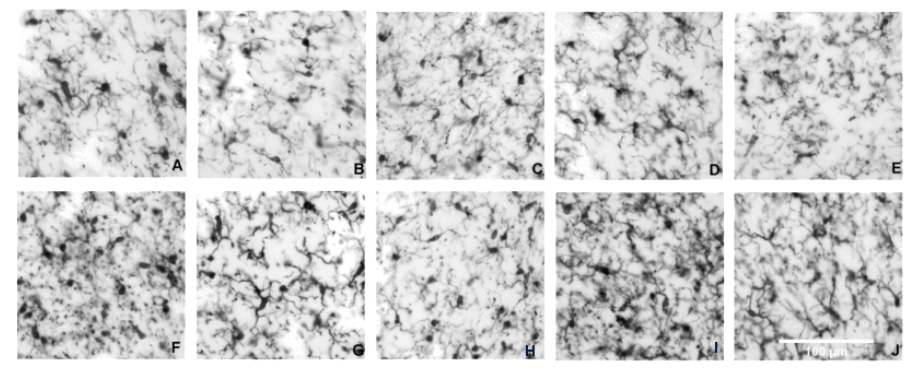

Microglial proliferation in the thalamus and stratum moleculare layer of the hippocampus

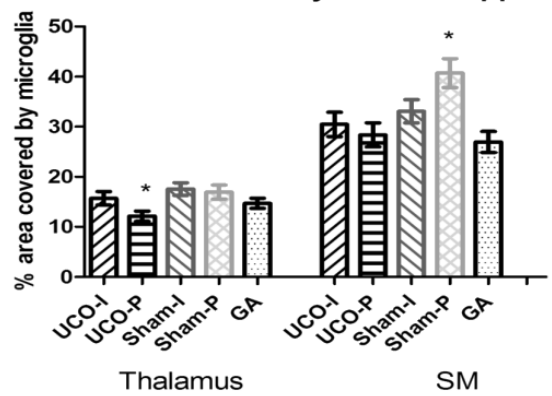

Figure 7.4: Microglial proliferation in the thalamus and stratum moleculare (S.M.) layer below the dentate gyrus of the hippocampus. The graph shows the mean \pm S.E.M. range of each group. In the thalamus, microglial proliferation was significantly lower in the UCO-propofol group than in the non-UCO exposed groups (shown with an *). In the S.M. the microglia was also lower in the UCO-groups than in the sham-propofol group. Overall, microglial proliferation was much higher in the hippocampal white matter than in the thalamus. Images were taken at $20 \mathrm{x}$ magnification. The scale bar represents $100 \mu \mathrm{m}$. Key: Thalamus: A: UCO-I, B: UCO-P, C: SH-I, D: SH-P, E: GA, S.M. of the DG: F: UCO-I, G: UCO-P, H: SH-I, I: SH-P, J: GA. 


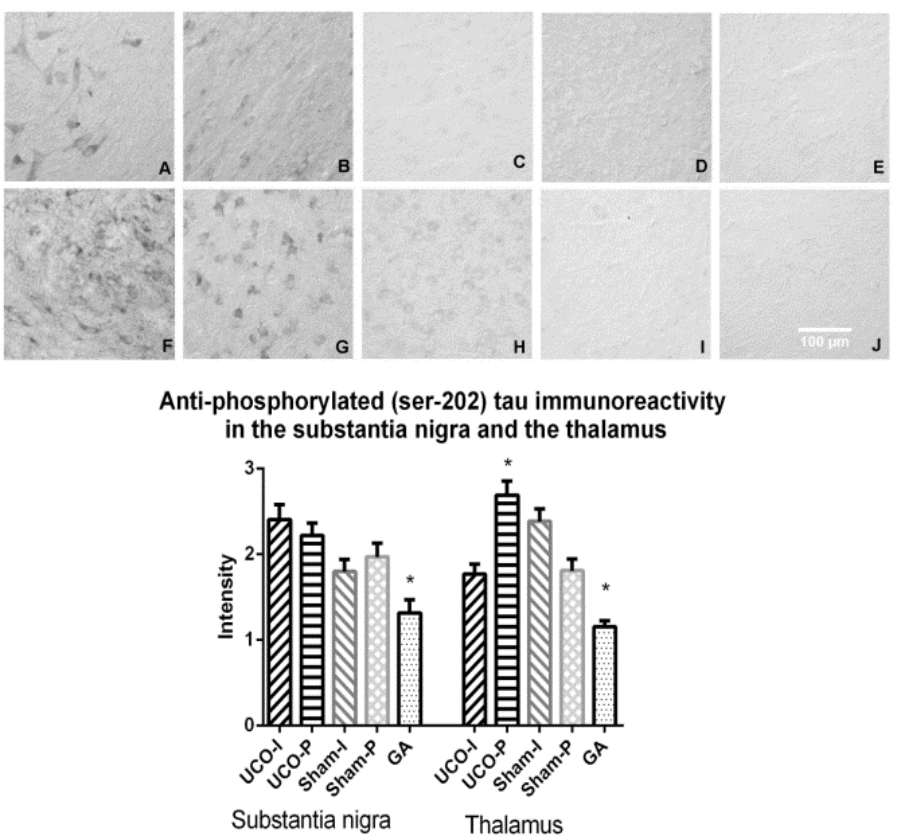

Figure 7.5: The intensity of anti-phosphorylated (ser-202) tau immunohistochemistry in the SN and thalamus. Photographs were taken at 10x magnification. Statistically significant differences in tau phosphorylation is indicated with an *. In both regions, the GA control had the lowest number of phosphorylation and both UCO and anesthesia exposure increased phosphorylation. In the thalamus, the greatest phosphorylation intensity was seen in the UCO-propofol group. The scale bar represents $100 \mu \mathrm{m}$. Key: SN: A: UCO-I, B: UCO-P, C: SH-I, D: SH-P, E: GA, Thalamus: F: UCO-I, G: UCO-P, H: SH-I, I: SH-P, J: GA

UCO-I/Sh-P p <0.001), and a significant difference in anesthesia-exposed groups and the GA control group $(1.23 \pm 0.06$, GA vs. all groups $\mathrm{p}<0.0001)$ (figure 7.5). Both UCO and anesthetic use increased the color-intensity of the CP-13 staining. In the hippocampus, the greatest changes were seen in the CA3 region with less reactivity in the SH-P (1.85 \pm 0.16$)$ and GA $(1.84 \pm 0.22)$ groups than in the UCO or isoflurane exposed groups (UCO-I 2.38 $\pm 0.13,2.30 \pm 0.11$ $\mathrm{SH}-\mathrm{I} 2.39 \pm 0.14, \mathrm{SH}-\mathrm{P}$ vs. UCO-I/SH-I $\mathrm{p}<0.05, \mathrm{SH}-\mathrm{P}$ vs. UCO-P $\mathrm{p}<0.1$, GA vs. UCO-I/SH-I $\mathrm{p}<0.1)$. In the D.G., only the difference between the UCO-P $(2.85 \pm 0.13)$ and GA $(2.16 \pm 0.16)$ reached statistical significance $(\mathrm{p}<0.05)$ (figure 6). The tau-ser-202 reactivity in the thalamus predominantly labelled the cell body, as seen in figure 7.6.

Changes in tau phosphorylation were less pronounced in the other regions 


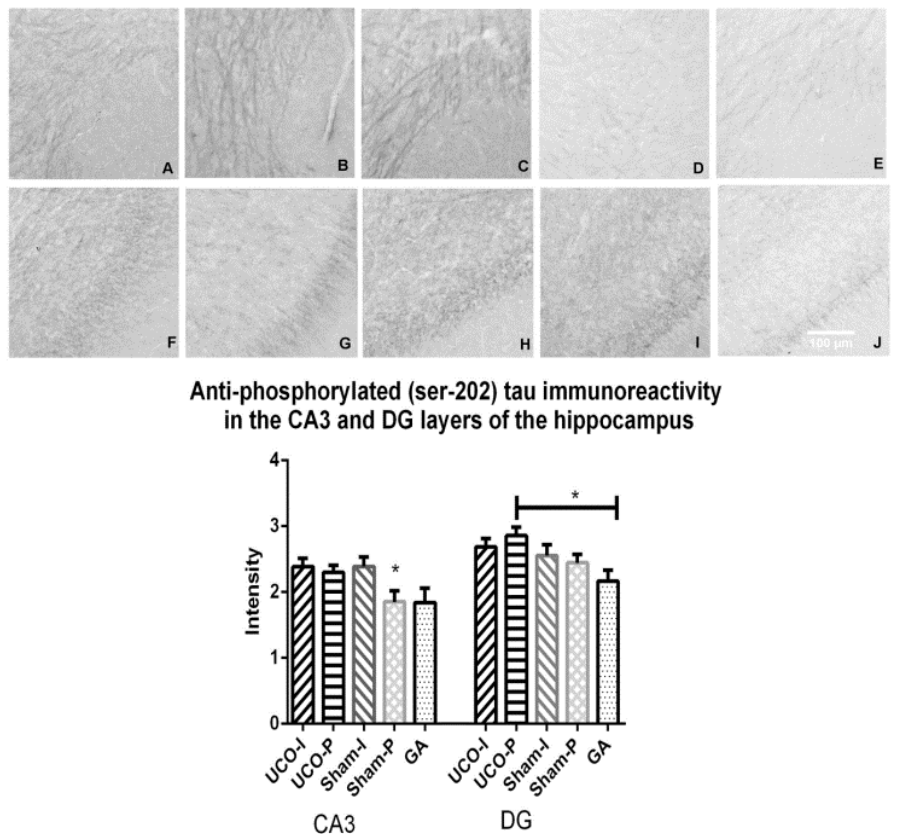

Figure 7.6: The intensity of anti-phosphorylated (ser-202) tau immunohistochemistry in the hippocampus CA3 and DG layers. The photographs were taken at 10x magnification. Statistically significant differences in tau phosphorylation is indicated with an $*$. In the CA3 region, both UCO and isoflurane-anesthesia increased CP-13 immunoreactivity, and the reactivity in the sham-propofol group was significantly lower. In the DG, the UCO-propofol had a significant increase in CP-13 reactivity in comparison to the GA control. The scale bar represents $100 \mu \mathrm{m}$. Key: CA3: A: UCO-I, B: UCO-P, C: SH-I, D: SH-P, E: GA; DG: F: UCO-I, G: UCO-P, H: SH-I, I: SH-P, J: GA 
tested, with no significant difference between groups seen in the fornix. The PHF-1 and MC1 antibodies did not detect any phosphorylated Ser396/Ser404tau or misfolded tau in our sheep tissue (data not shown).

\subsection{Discussion:}

In this study, we investigated whether the choice of anesthetic could limit the loss of dopaminergic neurons in the substantia nigra. The link between alterations of dopaminergic neurotransmission and HIE is well established. Studies in rodents suggest that mild HIE increases dopaminergic neurotransmission, whilst severe HIE reduces dopamine transmission. These changes persist in the weeks following the insult (Barkhuizen et al., 2017). Moreover, HIE damage to the $\mathrm{SN}$ in the fetal or neonatal rat resulted in lower dopaminergic neuron counts and locomotor impairment during adulthood (Burke et al., 1992, Strackx et al., 2008). A reduction in SN dopaminergic neurons in adulthood is characteristic of the degenerative disease Parkinson's disease. However, damage to the SN can manifest as movement disorders throughout life, such as in the pediatric-onset movement disorders, like Kufor-Rakeb syndrome, DOPA-responsive dystonia and the neurodegeneration with brain iron accumulation disorders (BekiesinskaFigatowska et al., 2013, DeLong and Wichmann, 2007). Children that had HIE, are also at increased risk of attention deficits, like ADHD (Van Handel et al., 2007). Structural abnormalities of the SN have been implicated in the pathology of ADHD (Romanos et al., 2010). Given the roles of the SN in movement and attentional deficits, reducing damage is clinically relevant. A previous study with this experimental paradigm suggested that propofol may provide neuroprotection via decreased apoptotic markers and increased anti-apoptotic markers the cerebral cortex (Seehase et al., 2015). In the substantia nigra, we showed more dopaminergic neurons in the SN of the UCO-propofol group than in the UCO-isoflurane group. Therefore UCO-isoflurane was neurotoxic than UCO-propofol. Propofol anesthesia may also have had a protective role in the $\mathrm{SN}$, but in the absence of a UCO group without anesthesia, this cannot be established. Due to animal welfare regulations, we did not include this group. Our study was only conducted at a single time-point 8 hours after the insult at a gestational age where the migration of dopaminergic neurons to the SN was incomplete in up to $50 \%$ of lambs per group. This prevented us from assessing the effect of these agents on net cell loss. Longer follow-up studies should determine whether the changes in neuron amounts persist after migration has completed.

In humans and sheep, the majority of the nervous system development 
occurs prenatally (van den Heuij, Wassink et al. 2016). In the early- and late preterm infant, the axonal pathways are developing and especially vulnerable to injury (Kostović and Jovanov-Milošević 2006). Under physiological conditions, the soluble microtubule-associated protein tau stabilizes axonal microtubule assembly. This is essential for the formation of the neuronal cytoskeleton. Phosphorylation of tau lowers its affinity for the microtubule lattice and thus disrupts its functions (Yu, Run et al. 2009, Spillantini and Goedert 2013).

The disruption of normal tau binding and abnormal tau distribution has been linked to a range of neuropathologies. In the neonatal setting, elevated serum tau is a general predictor of adverse outcomes after encephalopathies (Takahashi et al., 2014, Okumus et al., 2008). Tau is particularly well-known for its involvement in Alzheimer's disease, Parkinsonism and other late-life neurodegenerative diseases (Spillantini and Goedert, 2013). Propofol and other anesthetics have been associated with direct increases in tau phosphorylation, as well as indirect increases through hypothermia. This disease mechanism has been associated with an exacerbated cognitive decline post-operatively in the geriatric population (Whittington et al., 2013).

Hyperphosphorylated tau, at residues like serine-202, serine-396, and serine404, is the main component of the paired helical filament neuropathology in Alzheimer's disease, where phosphorylation of ser-202 precedes phosphorylation at ser-396/404 (Su et al., 1994, Spires et al., 2006). Tau is also phosphorylated at these sites during fetal development which enables the mobility of tau (Yu et al., 2009, Goedert et al., 1993, Bramblett et al., 1993, Spillantini and Goedert, 2013), but the extent of phosphorylation at these sites is greater in Alzheimer's disease than the developing brain ( $\mathrm{Yu}$ et al., 2009). In a healthy nervous system, and during development, tau is mainly confined to the axons, but in Alzheimer's disease, phosphorylated tau is also found in the dendrites and cell bodies (Goedert et al., 1993). We found marked phosphorylation in the cell bodies of groups exposed to anesthesia with or without additional UCO. This was particularly pronounced in the thalamus (see figure 5). Elevated tau phosphorylation was observed in the SN-region, thalamus and hippocampus indicating a widespread effect of the anesthetics across the brain. We selected additional regions, since in near-term and full-term infants with HIE, the most common site of injury is the central gray matter. The extent of damage to the basal ganglia and thalamus is a predictor of motor impairment after HIE (Counsell et al., 2010, Cabaj et al., 2012). The hippocampus is prone to injury after HIE (Volpe, 2012). It is also vulnerable to tau-mediated neurotoxicity as seen in Alzheimer's disease - and often affected by anesthesia-induced tau 
hyperphosphorylation (Whittington et al., 2013). We only observed immunoreactivity against early phosphorylation at ser-202 (CP13) in the fetal lambs, but no reactivity against misfolded tau (MC1) or the late-phosphorylation marker ser-396/ser-404 reactivity (PHF-1). The lack of PHF1-reactivity likely reflects that only the first indications of tau pathology were present since this antibody has previously been reported to show neurofibrillary tangle formation in sheep older than 5 years (Nelson et al., 1994). This is encouraging since early hyperphosphorylation may be reversible upon restoration of homeostasis (Van der Harg et al., 2014), and thus cautions, but does not necessarily limit the use of the anesthetics tested.

We have previously observed functional and molecular improvements with the combined ante- and postnatal treatment with the anesthetic administration to the maternal-fetal unit during UCO. Propofol prevented functional auditory impairment (Smit et al., 2013); reduced seizure activity (Seehase et al., 2011); and reduced apoptosis, reactive oxygen species and excitatory neurotransmitter receptors (Seehase et al., 2011, Seehase et al., 2015). In addition to their effects on glutamatergic receptors, propofol and isoflurane also has effects on several other neurotransmitter systems and ion channels including potentiation of the GABA-A and glycine receptors and inhibition of the nicotinic acetylcholine receptors (Rudolph and Antkowiak, 2004). In this study, we extended our investigation of potentially beneficial effects of anesthesia in these lambs to the microglial response. Microglia are the resident innate immune cells in the brain and play a central role in the initiation of an inflammatory response aimed at resolving injury caused by HIE. Persistent acute inflammation could be shifted to a chronic inflammatory state that may adversely affect brain development (Hagberg et al., 2012). Our results did not show a clear effect of the anesthetic selection on microglial response in the substantia nigra or other regions studied, and thus presumably these agents activate other neuroprotective pathways not related to neuronal inflammation. Surprisingly, we found lower microglial response in the UCO-groups, than in the sham groups, in the majority of the analyzed brain regions. This may indicate that anesthetic-use per se is detrimental, but it could limit the microglial response in the injured brain, as reported in earlier studies of focal brain injuries (Archer et al., 2017, Luo et al., 2013, Jander et al., 2000). Microglia are thought to contribute to secondary and tertiary phases of brain injury through the production of pro-inflammatory cytokines, proteases, complement factors, and excitotoxic amino acids. The microglial response initiates before 6 hours after the injury, but only peaks at 48-72 hours (Hagberg et al., 2012). Previous UCO studies on the fetal sheep at $70 \%$ or $85 \%$ of gestation found a significant microglial response in the white 
matter of the hippocampus, cortical and periventricular regions (Jellema et al., 2013). We measured microglial response in both white matter (fornix, S.O. of CA3 and S.M. of the D.G. in the hippocampus) and grey matter in the thalamus and $\mathrm{SN}$ region. In preterm infants, white matter injury is more common than grey matter injury due to the vulnerability of the developing oligodendrocytes. Microglial proliferation is thought to contribute to the white matter injury (Volpe et al., 2011, Cabaj et al., 2012). We may not have found increased microgliosis after UCO since, apart from the hippocampal regions, we focused on the grey matter and our lambs were sacrificed before the bulk of the microglial response.

\subsection{Conclusion:}

In this study, we showed that the choice of anesthetic could limit tyrosinehydroxylase expression loss in the substantia nigra and that the choice of the anesthetic agent played a key role in limiting neuronal loss. Although we could not ascertain whether this signifies reduced neurotoxicity of UCO-propofol, or propofol-mediated neuroprotection, these findings are clinically relevant, given the susceptibility of the SN to the insult and its role in locomotion and attention deficits. This beneficial effect did not appear to be mediated through the microglia, although the lambs were sacrificed before the bulk of the microglial response. We further noted a possible pathogenic distribution of phosphorylated tau in this model after exposure to the anesthetics, which was aggravated by the insult. This effect could be detrimental if it persisted and longer term studies on this subject are needed.

\subsection{Acknowledgements:}

The authors would like to acknowledge Dr. Peter Davies and the Albert Einstein College of Medicine for the generous gift of the anti-tau antibodies, Dr. Fred van Leeuwen for consulting on the tau staining and the generous gift of the Alzheimer's tissue and Hellen Steinbusch for her excellent technical assistance. This research was partially supported by the Sistema de Investigación y Desarrollo (SINDE) of the Universidad Católica de Santiago de Guayaquil, Guayaquil, Ecuador, through the grant No SIU- 319: Perinatal asphyxia and stem cell treatment. M. Barkhuizen is funded by the National Research Foundation of South Africa (Grant specific reference number 98217) and the Foundation of Pediatrics, Maastricht University Medical Center + . All views expressed in this article are those of the authors and not of the funding agencies. 


This chapter is embargoed at request

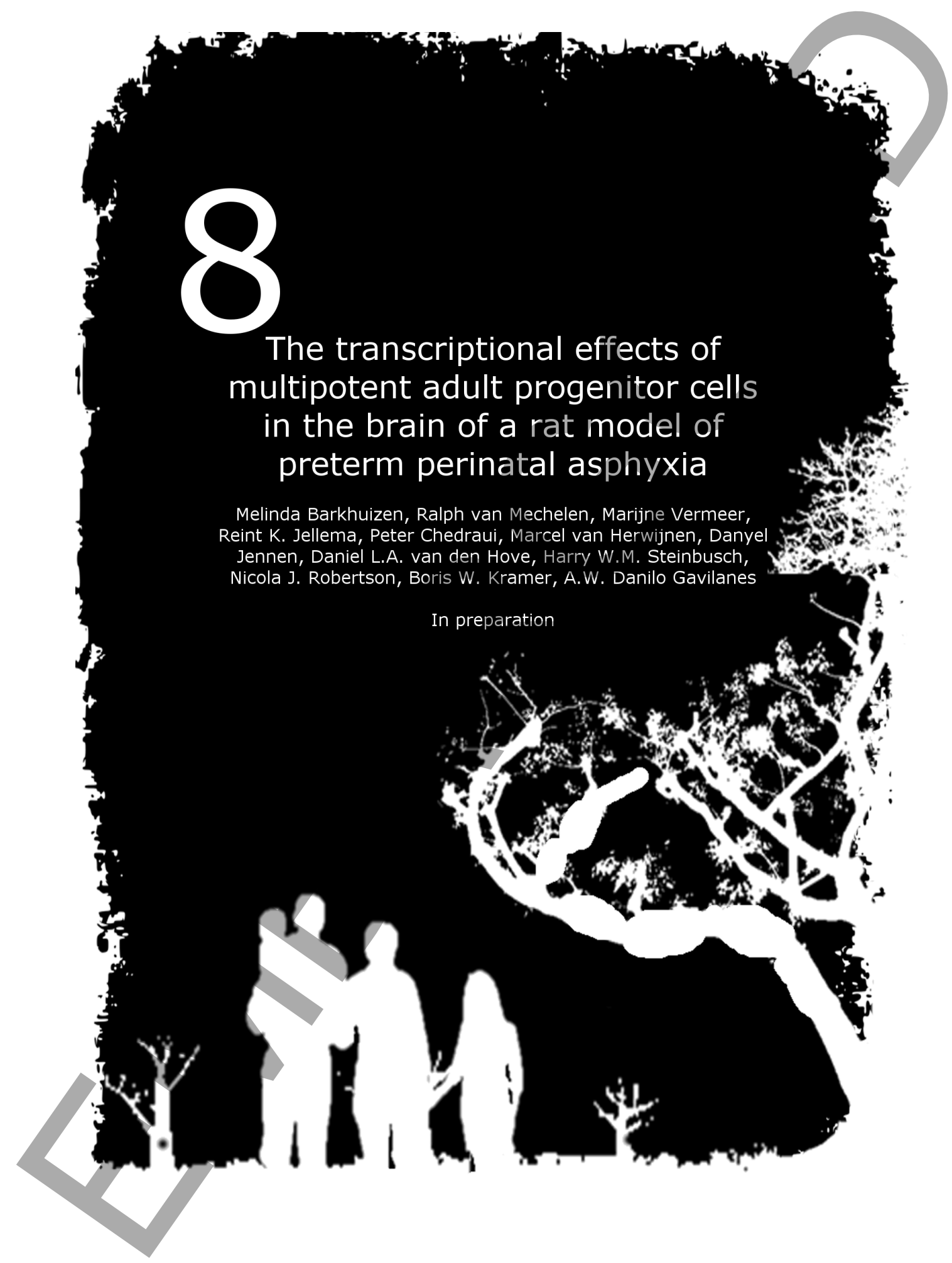


This chapter is embargoed at request

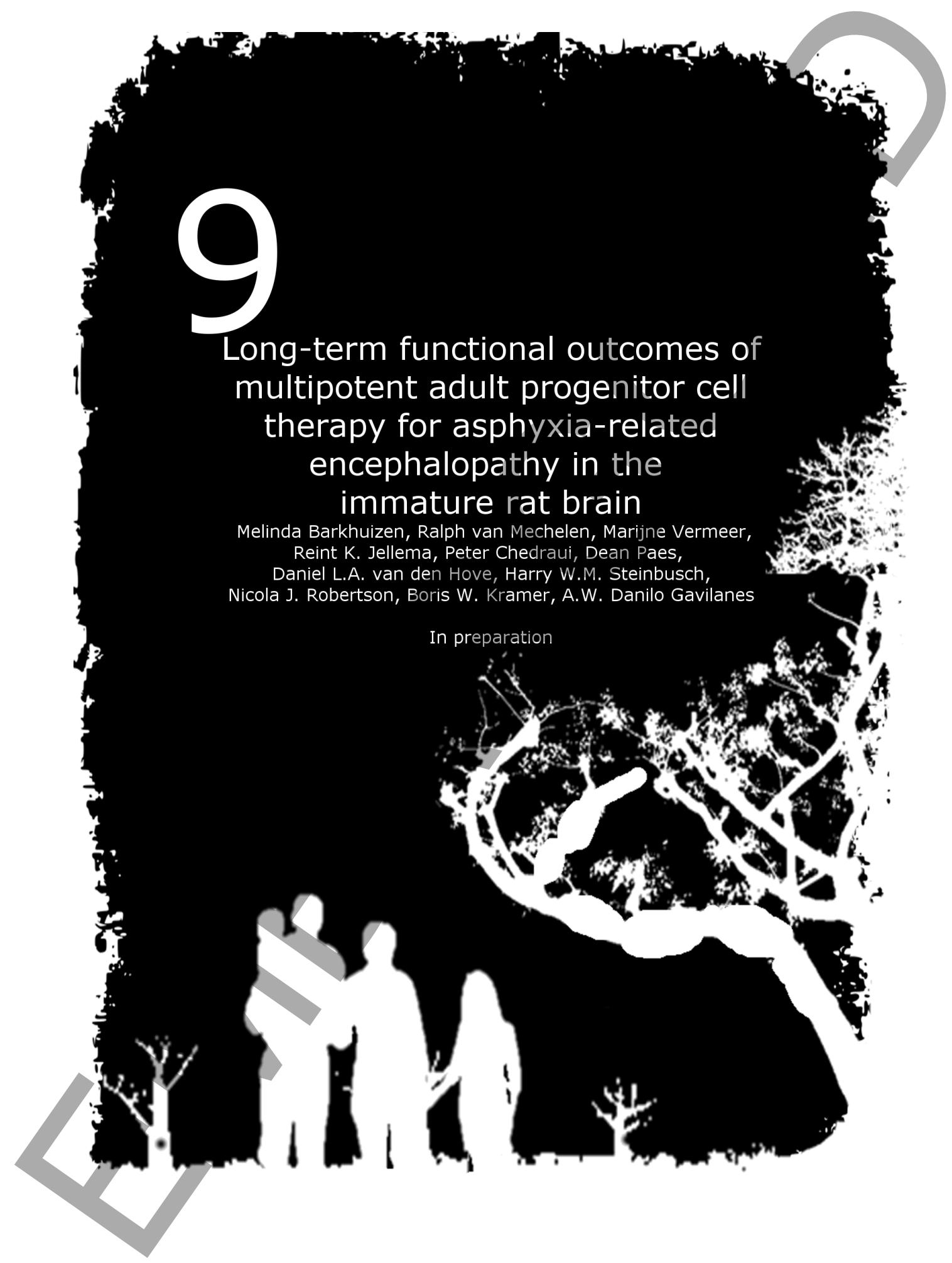




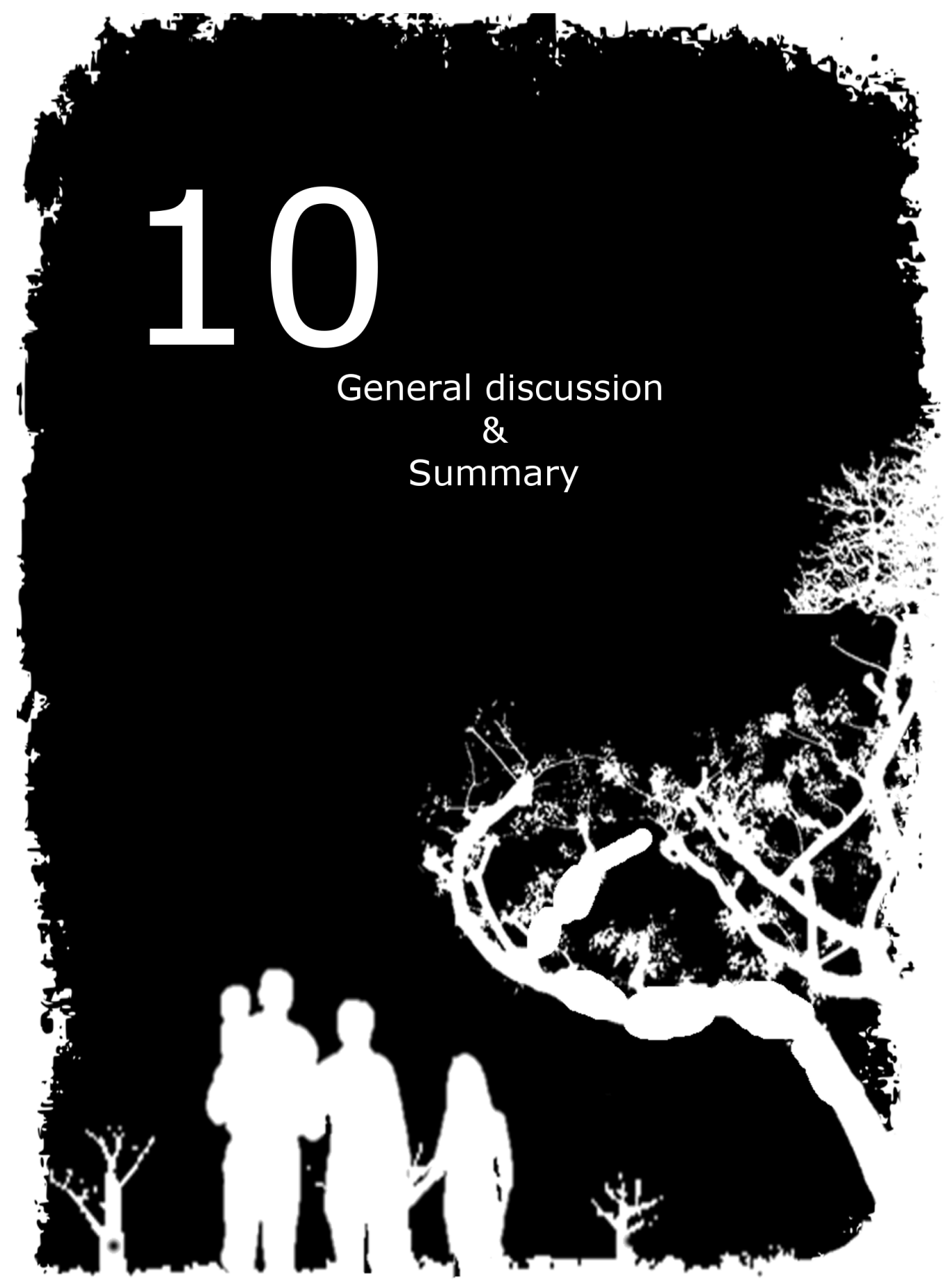




\section{General discussion and summary}

\subsection{General pathological hallmarks of age-related neurodegenerative diseases and the overlap with peri- natal asphyxia}

\subsubsection{Discussion:}

Parkinson's disease (PD), Huntington's disease (HD), and several other common neurodegenerative diseases like Alzheimer's disease (AD), are characterized by the death of neurons in specific brain regions, accompanied by deposits of misfolded proteins. PD and HD represent three major classes of proteinopathies, namely: the tauopathies $(\mathrm{PD}, \mathrm{AD}$, frontotemporal dementia, progressive supranuclear palsy, corticobasal degeneration), synucleinopathies (PD and multiple system atrophy) and repeat expansion disorders (HD, Frontotemporal Dementia, Amyotrophic Lateral Sclerosis, Spinocerebellar Ataxias). The clinical diagnosis does not always predict the underlying neuropathology and many patients have more than one type of proteinopathy (Kudo and Takeda 2007, Kovacs 2016). Whilst it is known that genetic variants can cause or predispose to the proteinopathies, the majority of cases have a sporadic presentation with unknown etiology. It has been proposed that sporadic neurodegenerative disease is due to the cumulative effects of several genetic and environmental insults over the course of a lifetime (Sulzer 2007).

These insults may precede the symptom onset by several years since the neurodegenerative diseases have a long latent period (Salat, Noyce et al. 2016). Insults early in life could increase susceptibility to a secondary insult years later that initiates the disease process through epigenetic modifications (Lahiri and Maloney 2010). Even in HD, a mid-life onset disease caused by a single genetic insult, early-life insults are important for disease development. The selective expression of the damaging mutant gene product limited to the developmental period in mice is sufficient to cause symptomatic disease in adulthood (Molero, Arteaga-Bracho et al. 2016). These insults are often overlooked due to their long latency until disease onset, but early-life factors could play an important role in late-life sporadic neurodegeneration (Lahiri and Maloney 2010).

In addition to misfolded proteins, chronic low levels of several general neurotoxic processes have been implicated in the pathology of neurodegenerative diseases (Kovacs 2016). These processes also characterize the primary and secondary phases of acute neuronal injury after perinatal asphyxia (PA). In the first 6 hours after PA, an acute brain injury is caused by the combined effects 
of cellular energy failure, acidosis, excitotoxicity due to excessive glutamate release, intracellular calcium accumulation, lipid peroxidation, and nitric oxide neurotoxicity that disrupts essential components and processes of the cell, resulting in death. A secondary cerebral energy failure occurs from 6 to 48 hours after the primary event and may involve mitochondrial dysfunction secondary to extended reactions from primary insults (calcium influx, excitatory neurotoxicity, oxygen free radicals, or nitric oxide formation). A tertiary phase of neuronal loss occurs in the days to months after recovering from the insult due to supra-physiological increases in apoptosis, persistent inflammation and epigenetic changes. The basal ganglia are particularly vulnerable to these insults (Perlman 2006, Morales, Bustamante et al. 2011, Davidson, Wassink et al. 2015). Chronic exposures to low-grade excitotoxicity, oxidative stress, free radicals, nitric oxide and mitochondrial dysfunction and sustained inflammation are also associated with the neuropathology of the neurodegenerative diseases (Pérez-Severiano, Escalante et al. 2002, Fan and Raymond 2007, Oliveira, Jekabsons et al. 2007, Ehrlich 2012).

There are several specific mechanisms shared between PA and HD. The striatal medium spiny neurons receive the highest co-transmission of glutamatergic input (from the cortex) and dopaminergic input (from the substantia nigra pars compacta) among neuronal subtypes. It has been proposed that the combination of excessive glutamate and dopamine release is responsible for the hyperkinesia early in the disease, and could contribute to the vulnerability of the medium spiny neurons to the expanded HD allele (André, Cepeda et al. 2010, Ehrlich 2012). Both of these neurotransmitters are also increased after perinatal asphyxia. Shortly after the insult, there is an excessive glutamate release which leads to excitotoxicity. Dopamine is also increased shortly after mild- moderate asphyxia and increased dopamine release was sustained into adulthood in models of global perinatal asphyxia (Barkhuizen, Van den Hove et al. 2017). In human cohorts, the lasting abnormalities in dopamine neurotransmission after a perinatal insult was reflected in the epidemiological observations that obstetric insults increase the risk of developing psychoses (Laurens, Luo et al. 2015) and ADHD (Smith, Schmidt-Kastner et al. 2016).

The pathology of both diseases has also been linked to an over-activation of the DNA repair pathways. In HD, somatic instability of the CAG-repeat expansion occurs in post-mitotic neurons. This is particularly prominent in the striatum and cortex, which are severely affected by HD. Large somatic expansions in these regions have been associated with a decreased age-of-disease onset (Swami, Hendricks et al. 2009). The somatic expansions are thought to 
occur during the process of DNA mismatch repair. Repetitive DNA sequences can form unusual secondary structures which attract the DNA mismatch repair enzymes. A number of enzymes with the ability to nick DNA, and therefore necessitate DNA repair, promote CAG expansion and were associated with HD and other polyglutamine repeat disorders in genome-wide association studies (Bettencourt, Hensman-Moss et al. 2016). PA compromises the integrity of the genome, which triggers the activation of sentinel proteins that maintain genome integrity, such as poly (ADP-ribose) polymerases (PARPs). The PARPs interact with DNA polymerase and DNA repair enzymes, which improves survival of the cells. Despite these beneficial effects, excessive activation of the PARP-1 pathway signals irreparable DNA damage which initiates cell death (Morales, Bustamante et al. 2011).

In this thesis, I explored the hypothesis that the vulnerability of the basal ganglia to genetic and perinatal insults could accelerate the presentation of a movement disorder in individuals with both insults. I focused on HD as an example of striatal degeneration caused entirely by a genetic defect, with a modifiable onset; and PD as an example of mostly sporadic neurodegeneration in the substantia nigra. Sporadic PD is due to the cumulative effects of environmental insults and genetic factors, such as glucocerebrosidase mutations. These two conditions are currently incurable, but the symptoms can be managed. Predicting individuals who are at risk of the disease and their likely age-ofdisease onset is crucial for efforts to reduce the burden of neurodegenerative disease (Salat, Noyce et al. 2016). In this thesis, I confirmed that perinatal insults reduce the age-of-diagnosis of HD with epidemiological data.

This led to the second hypothesis, that preventing basal ganglia damage shortly after the insult could reduce the disability burden later in life. I tested the efficacy of initiating glutamate-antagonism therapy to the fetus in distress to reducing damage to the substantia nigra, and the efficacy of administering multipotent adult progenitor cells within 24 hours after the insult to prevent functional deficits in this thesis. Figure 10.1 gives an overview of the themes studied in this thesis. 


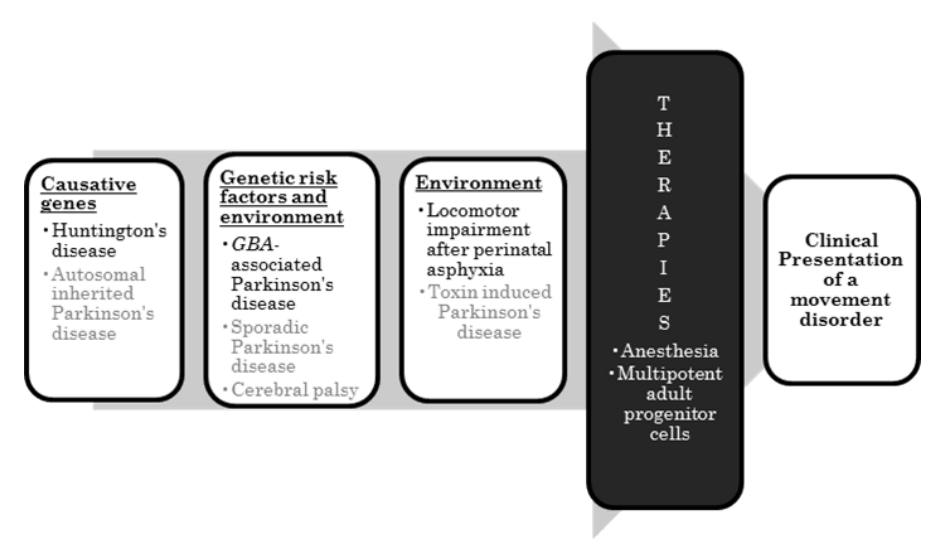

Figure 10.1: The genetic- environmental continuum of basal ganglia insults studied.

\subsubsection{Summary:}

In Chapter 1, I introduced the main topics of this thesis, namely genetic and perinatal insults, basal ganglia damage and movement disorders. Furthermore, the aims of the different chapters were summarized.

My first aim was to study the genetic contributions to basal ganglia damage. In chapter 2, additional mechanisms how $G B A$ mutations to the pathogenesis of Parkinson's disease were reviewed. GBA variants affect the lysosome's ability to clear the sphingolipid glucocerebroside, which causes an accumulation of this product and reduces the lysosomes overall ability to clear several pathological proteins. These proteins accumulate and cause stress in several molecular pathways, which eventually causes the death of substantia nigra neurons during Parkinson's disease. GBA variants were associated with an increased risk of Parkinson's disease and a more rapid progression of the disease. In chapter 3, it was shown that $G B A$ variants are a common genetic risk factor for Parkinson's disease in the South African Caucasian population. The prevalence of $G B A$ variants in this population was comparable to the prevalence in the founder European populations.

My second aim was to determine whether genetic factors interact with perinatal factors to aggravate movement disorders on a phenotypical and molecular level. In chapter 4, it was shown that perinatal insults and neurodevelopmental disorders can interact with the genetic background to reduce the median age-of-diagnosis of Huntington's disease by $4-6$ years in two large epidemiological cohorts. The HD genotype per se did not appear to increase the risk of neurodevelopmental disorders after perinatal insults, since there was minimal 
overlap between the participants which had a perinatal insult and participants which had a neurodevelopmental disorder. The incidence of perinatal insults and neurodevelopmental disorders was lower in the HD-cohorts than in the general population. This likely reflected the difficulties of ascertaining early-life insults retrospectively in general cohort studies and underreporting, rather than a true lower incidence among persons with HD gene expansions.

The mechanisms of neurotoxicity following PA in susceptible brain regions and behavioral outcomes in a rat model were reviewed in chapter 5. I further discussed how PA caused long-term changes in the expression of several neurotransmitter systems, particularly dopaminergic neurotransmission. An imbalance in dopaminergic transmission underlies the pathology of hypokinetic and hyperkinetic movement disorders, as well as psychosis. The adverse behavioral outcomes seen on locomotion, cognition and anxiety in this model and its suitability to test interventions for perinatal asphyxia were also reviewed.

In chapter 6, I showed that the striatum developed greater supra-physiological increases in nitric oxide release and peroxynitrite formation after PA than the cerebellum. The therapeutic window to reduce excessive NO release is within the first 8 days after the insult. These results provided both potential therapeutic insights for PA and a potential mechanism how PA and HD can interact on the molecular level. Degeneration of the medium spiny neurons in the striatum is the pathological hallmark lesion of HD, although the disease also affects the cerebellum and several other brain regions (Rüb, Vonsattel et al. 2015). Nitrosidative stress is thought to be one of the mechanisms contributing to the selective death of the striatal medium spiny neurons in HD (Ehrlich 2012).

In chapter 7, focussed on whether PA could increase tau hyperphosphorylation. Tau hyperphosphorylation has been implicated in the pathology of several neurodegenerative diseases, including PD and AD (Compta, Parkkinen et al. 2014). Tau phosphorylation was investigated in the substantia nigra and the hippocampal regions, which are affected by dementias like AD. PA in combination with anesthetic use generally increased tau phosphorylation in several brain regions, including the thalamus and hippocampal areas. PA increased the loss of dopaminergic neurons in the substantia nigra - another pathological hallmark of PD. The main limitation of this study was that it was performed at 8 hours after the insult. Long-term studies are needed to see whether these effects persist and contribute to late-life neurodegeneration. This chapter concluded the research into the etiology of basal ganglia damage presented in this thesis. 


\subsection{Therapeutic interventions:}

\subsubsection{Discussion:}

Currently, neurodegenerative diseases have no cure or effective disease modifying therapy. Therapies for the neurodegenerative diseases are limited to symptomatic relief. Successful disease modification approaches implemented at the very early stages of pathology might change the way these diseases are managed in the future (Salat, Noyce et al. 2016). Interventions which could limit the damaging effects of early-life insults are needed to limit both the early-life disability burden and the contribution of perinatal insults to late life neurological disability. In the remainder of this thesis, I investigated therapies for PA in the preterm infant. At this time, there are no FDA approved therapies for preterm infants with PA. For term infants, therapeutic hypothermia or selective head cooling is widely used to reduce the mortality and morbidity after PA. This has also been trialed in late-preterm infants from 34-35 weeks of gestation (Rao, Trivedi et al. 2016), but a small pilot study in preterm infants from 32-35 weeks highlighted safety concerns when using these practices in preterm infants (Walsh, Butler et al. 2015). For the very-low gestational age (VLGA) neonates, below 32 weeks, there are no therapies available.

\subsubsection{Summary:}

In chapter 5, potential therapeutic strategies that have been tested in a rat model of PA in the VLGA infant were reviewed. These include therapeutic hypothermia, as well as glutamate antagonism (Herrera-Marschitz, Loidl et al. 1993, Engidawork, Loidl et al. 2001) and several other studies with pharmacological agents that target specific pathways. Some of these compounds have proceeded to clinical trials in term PA infants, but currently, none of them are FDA approved yet. In chapter 7, I investigated whether reducing excitotoxicity with anesthetics which antagonize the glutamatergic NMDA-receptor could attenuate dopaminergic neuron loss in a sheep model of near-term PA. Propofol had greater neuroprotective capabilities than isoflurane and that the choice of anesthetic for an emergency C-section could reduce some of the neuropathology. I also showed that anesthetics increased tau-phosphorylation and should be used cautiously in combination with other therapeutics that also increase phosphorylation.

There is a growing interest in the ischemia field to use biological molecules that can exert several beneficial actions, particularly stem cell products. The 
mesenchymal stem cells (MSCs), a type of bone marrow-derived stem cell, have shown promising behavioral, structural and biochemical effects in various models of neonatal stroke and global asphyxia. These include an attenuated peripheral immune reaction and increased levels of trophic factors which increase neuronal survival (van Velthoven, Kavelaars et al. 2012, Donega, van Velthoven et al. 2013, Jellema, Wolfs et al. 2013). Another type of bone marrow-derived cell, the multipotent adult progenitor cell (MAPC), have shown greater immunomodulatory potency and efficacy in models of traumatic brain injury than the MSCs (Walker, Bedi et al. 2012, Jacobs, Roobrouck et al. 2013). The MAPCs are smaller than the MSCs, which could potentially reduce their first-pass metabolism and allow more cells to reach the site of injury after systemic administration (Fischer, Harting et al. 2009). These cells have also shown beneficial immunomodulatory effects in the sheep model of acute global PA in the preterm brain (Jellema, Ophelders et al. 2015).

In chapter 8 and $\mathbf{9}$, I extended the preclinical testing of these cells to the rat model of PA in the VLGA infant. In chapter $\mathbf{8}$, key transcripts and pathways responsible for the modulating effect of multipotent adult progenitor cells (MAPCs), a stem cell product, were identified in 2 week old rats. The molecular effect of these cells was determined by the route of administration. Intranasal administration of these cells results in thousands of changes to the brain transcriptome, whilst the systemic administration route resulted in less direct effects on the gene transcription in the brain. Functional analysis of the transcripts showed that pathways involved in processes such as DNA repair, G-protein coupled receptors, synapse reorganization, and cellular proliferation were relevant to the pathology of PA and the effects of MAPCs. In chapter 9, I investigated whether the molecular effects of systemic MAPC therapy seen in the two week old rats translate into functional improvements later in life. The systemic MAPC-therapy was more effective in female rats than in male rats. The therapy had a partial effect on recognition memory and a normalization of hyperlocomotion in adult males. Survivors of PA often have some degree of cognitive deficits and a therapy that boosts cognition is of societal relevance. The pro-cognitive effect could potentially be strengthened further in combination with other neuroprotective strategies as a much needed clinical intervention for these infants.

In conclusion, in this thesis, I explored how perinatal insults and genetic factors affect the basal ganglia and the functional consequences of the insults and their interactions. I generated molecular hypotheses and tested therapeutic strategies to limit the damage after PA. Future research needs to follow these 
hypotheses over longer time-periods and combine PA with transgenic models to truly study the effect of interactions between PA and predisposing genetic factors. The therapeutic strategies tested (anesthetic use and MAPCs) both showed beneficial effects on some of the functional domains and molecular targets investigated. Future research should investigate methods to further enhance these effects. 


\subsection{Nederlandse samenvatting}

In dit proefschrift heb ik genetische en perinatale factoren onderzocht die de basale ganglia beschadigen - een verzameling hersengebieden die betrokken zijn bij motorcoördinatie. Schade aan de basale ganglia resulteert in bewegingsstoornissen bij kinderen, zoals hersenverlamming, en aandoeningen bij volwassenen, zoals de ziekte van Parkinson en de ziekte van Huntington. Uiteindelijk heb ik therapieën getest om de basale ganglia-schade en motorische beperking te verminderen na een perinatale hersenbeschadiging.

In hoofdstuk 1 introduceerde ik de hoofdonderwerpen van dit proefschrift, namelijk genetische en perinatale factoren, basale ganglia schade en bewegingsstoornissen. Verder werden de doelstellingen van de verschillende hoofdstukken samengevat.

Het eerste deel van dit proefschrift legt de nadruk op genetische risicofactoren. In hoofdstuk 2 heb ik samengevat hoe variaties in het $G B A$-gen, wereldwijd de meest voorkomende groot effect risico-variant voor de ziekte van Parkinson, bijdraagt tot de pathologie van de ziekte en de klinische gevolgen. $G B A$-varianten beïnvloeden het vermogen van het lysosoom om het sphingolipide glucocerebroside te verwijderen, een ophoping van dit eiwit plaatsvindt. Hierdoor wordt het totale vermogen wordt verminderd om verschillende pathologische eiwitten te elimineren, waaronder $\alpha$-synuclein. De accumulatie van $\alpha$-synucleine in de substantia nigra en de daaropvolgende neurologische dood is een pathologisch kenmerk van de ziekte van Parkinson. GBA varianten zijn geassocieerd met een verhoogd risico op het ontwikkelen van de ziekte van Parkinson en een snellere progressie van de ziekte. In hoofdstuk 3 bevestigde ik dat $G B A$-varianten een veel voorkomende genetische risicofactor zijn voor de ziekte van Parkinson in de Zuid-Afrikaanse Kaukasische bevolking. De prevalentie van $G B A$ varianten in deze populatie was vergelijkbaar met de prevalentie in de Europese bevolkingen.

Mijn volgende onderzoeksvraag was of er een interactie bestaat tussen genetische factoren die de basale ganglia en perinatale beschadiging beïnvloeden. De ziekte van Huntington is een autosomale dominante bewegingsstoornis veroorzaakt door een verlies van neuronen in het striatum. De mutatie dragers van de ziekte van Huntington zullen de ziekte altijd ontwikkelen, maar de aanvang kan door milieu factoren worden beïnvloed. In hoofdstuk 4 heb ik aangetoond dat perinatale verwondingen en neurodevelopmentale aandoeningen de leeftijd waarop de ziekte van Huntington word gediagnosticeerd verminderen 
in twee grote epidemiologische cohorten van de ziekte.

In hoofdstuk 5 ben ik nagegaan hoe een veel voorkomende perinatale oorzaak van hersenbeschadiging, perinatale asphyxia (PA) neurologische schade veroorzaakt in een representatief ratmodel. PA veroorzaakt langdurige veranderingen in de concentraties van verschillende neurotransmittersystemen, in het bijzonder het dopaminergische neurotransmissiesisteem. Een disbalans van dopaminergische neurotransmissie ligt ten grondslag aan de pathologie van de ziekte van Parkinson en Huntington, evenals bij psychose. Ik heb samengevat hoe het model ons begrip van de moleculaire gevolgen van PA heeft verbeterd; welke functionele tekorten aanwezig waren en de geschiktheid van dit model om therapieën voor PA te testen aangetoont.

In hoofdstuk 6 bleek dat er in de eerste twee weken na de PA regionale verschillen waren in de suprafysiologische productie van stikstofoxide. Het striatum had grotere toenames in de overmaat aan stikstofoxideproductie dan het cerebellum, vooral in de eerste acht dagen na de verwondingen. Beide regio's zijn bekend om kwetsbaar te zijn voor PA en de ziekte van Huntington. Onze resultaten suggereren het aanpakken van de productie van stikstofmonoxide na PA belangrijk kan zijn om de basale ganglia-schade te verminderen. Dit hoofdstuk sluit het onderzoek naar de etiologie van de basale ganglia schade die in dit proefschrift wordt voorgesteld af.

In de volgende hoofdstukken onderzocht ik farmacologische en biologische interventies die de PA-schade aan de basale ganglia zouden kunnen verzwakken. In hoofdstuk $\mathbf{7}$ heb ik aangetoond dat het modulerende van de excitotoxiciteit met vaak gebruikte anesthetica het dopaminergische neuronverlies in de substantia nigra kan verminderen zonder de microgliale reactie te veranderen. Deze therapie verhoogde echter ook acute tau fosforylering in de substantia nigra en andere regio's. Deze tau-hyperfosforylering werd ook gezien in de thalamus, een extra regio dat betrokken is bij bewegingscontrole en in de hippocampus. De hippocampus is belangrijk voor cognitie en bevat tau-pathologie bij dementie. Doorlopende hyperfosforylering van tau kan de neuronale dood verergeren.

In hoofdstuk 8 onderzocht ik de effecten van Multipotente Volwassen Voorganger Cel (MVVC) therapie op heel genoom transcriptie in de hersenen van twee week oude ratten, die kort na de perinatale hersenbeschadiging de therapie kregen. MVVC's zijn een allogene stamcellenproduct, die veelbelovende resultaten hebben getoond in knaagdiermodellen van hersenletsel, maar hun werkingsmechanisme is grotendeels onbekend. Ik heb aangetoon dat pathways 
die verband houden met DNA-reparatie, cellulaire proliferatie en synapsreorganisatie betrokken waren bij het therapeutische effect en de toedieningsweg voor de MVVC's en dat ze het transcriptieprofiel van de hersenen beïnvloedde 2 weken na toediening.

In hoofdstuk 9 onderzocht ik of de MVVC therapie functionele tekorten kunnen voorkomen in adolescente en volwassen ratten die PA hadden. Ik heb aangetoond dat de therapie de cognitieve tekorten gedeeltelijk herstelde, zelfs bij volwassen ratten en de hyperlocomotie bij volwassen mannelijke ratten verlaagd, maar het kon nie emotionele tekorten voorkomen.

In hoofdstuk 10 heb ik met algemene discussie mijn onderzoek afgesloten. In dit proefschrift hebben we aangetoond dat vroegtijdige beschadigingen, zoals $\mathrm{PA}$, een langdurig effect hebben op motorische complicaties later in het leven. Deze tegenslagen zijn een belangrijke risicofactor voor motorische complicaties in de algemene populatie - en bij personen die genetisch aanleg hebben voor bewegingstoornissen. Het beperken van de langdurige schadelijke effecten van PA is van cruciaal belang om de handicapbelasting van bewegingsstoornissen gedurende het leven te verminderen. We hebben gunstige effecten getoond van een therapie tegen excitotoxiciteit en stamceltherapie gericht op verschillende moleculaire doelen. Deze therapieën kunnen voor PA preklinisch verder onderzocht worden. 


\subsection{Afrikaanse opsomming}

In hierdie proefskrif het ek genetiese en perinatale faktore ondersoek wat die basale ganglia, 'n versameling breinstreke betrokke by motoriese koördinasie, beskadig. Skade aan die basale ganglia lei tot pediatriese bewegingsteurnisse, soos serebrale lamheid, en volwasse bewegingsteurnisse soos Parkinson en Huntington se siektes. Ek het verder terapieë getoets om skade aan die basale ganglia en motoriese beperkings te verminder na 'n perinatale besering.

In hoofstuk 1 is die hoofonderwerpe van hierdie proefskrif bekendgestel, naamlik genetiese en perinatale beserings, basale ganglia-skade en bewegingssteurnisse. Verder is die doelstellings van die verskillende hoofstukke opgesom.

Die eerste gedeelte van hierdie proefskrif het op genetiese risikofaktore gefokus. In hoofstuk 2 het ek nagegaan hoe variasie in die $G B A$ geen, wêreldwyd die mees algemene groot effek risiko variant vir Parkinsons, bydra tot die patologie en kliniese uitkomste. $G B A$ variante beïnvloed die lisosoom se vermoë om die sfingolipied glukoserebrosied af te breek, wat akkumuleer en die vermoë om verskeie patologiese proteïene, insluitend $\alpha$-synukleïen, te verwyder verminder. Die akkumulasie van $\alpha$-synukleïen in die substantia nigra, en die gevolglike verlies aan neurone is 'n patologiese kenmerk van Parkinson se siekte. GBA-variante word geassosieer met 'n verhoogde risiko van Parkinsons én 'n vinniger progressie van die siekte. In hoofstuk 3 is dit bevestig dat $G B A$-variasie 'n algemene genetiese risikofaktor vir Parkinson se siekte in die Kaukasiese Suid-Afrikaanse bevolking is. Die voorkoms van $G B A$-variasie in hierdie bevolking was vergelykbaar met die voorkoms in die stigter Europese bevolkings.

My volgende navorsingsvraag was of daar 'n interaksie tussen genetiese faktore is wat die basale ganglia en perinatale beserings. Huntington se siekte is 'n autosomale dominante bewegingssteurnis wat veroorsaak word deur 'n verlies van neurone in die striatum. Draers van die Huntington mutasie sal altyd die siekte ontwikkel, maar die aanvang van die siekte kan deur omgewingsfaktore beïnvloed word. In hoofstuk 4 is dit getoon dat perinatale beserings en ontwikkelingsversteurings die ouderdom van diagnose van Huntington se siekte verlaag in twee groot epidemiologiese studies.

In hoofstuk 5 is beskryf hoe 'n algemene perinatale besering, perinatale asfyksie (PA), neurologiese skade veroorsaak in 'n verteenwoordigende rotmodel van dié besering. PA veroorsaak langtermyn veranderinge in die vlakke verskeie 
neurotransmissiesisteme, veral dopaminergiese neurotransmissie. 'n Wanbalans in dopaminergiese neurotransmissie is onderliggend aan die patologie van Parkinson en Huntington se siekte, en psigoses. Ek bespreek verder hoe die model ons begrip van die molekulêre gevolge en funksionele steurnisse na PA verbeter het en die geskiktheid van die model om terapieë te ontwikkel.

In hoofstuk 6 het ek gewys dat daar streeksverskille in suprafisiologiese stikstofoksiedproduksie is in die eerste twee weke na PA. Die striatum het groter toenames oormatige stikstofoksiedproduksie as die serebellum gehad, veral in die eerste agt dae na die besering. Albei gebiede is kwesbaar in beide PA en Huntington se siekte. Ons resultate stel voor dat die produksie van stikstofoksied geteiken kan word na PA om basale ganglia-skade te verminder. Hierdie hoofstuk het die navorsing oor die etiologie van basale ganglia-skade wat in hierdie proefskrif aangebied word afgesluit.

In die volgende hoofstukke het ek farmakologiese en biologiese strategieë ondersoek wat die skade aan die basale ganglia na PA kan verminder. In hoofstuk $\mathbf{7}$ is bevind dat die verlaging van eksitotoksisiteit, met algemeen gebruikte narkosemiddels, dopaminergiese neuronverlies in die substantia nigra kan beperk sonder om die mikroglia respons te beïnvloed. Hierdie terapie het egter ook akute tau fosforilering in die substantia nigra en ander streke verhoog. Die hipergefosforileerde tau is ook in die thalamus waargeneem, nog 'n streek wat betrokke is by die beheer van beweging, en in die hippokampus. Die hippokampus is belangrik vir geheue en denke, en dit word veral geaffekteer deur tau-patologie in dementie. Voortgesette tau hiperfosforilering kan neuronverlies vererger.

In hoofstuk $\mathbf{8}$ is ondersoek ingestel na die effek van Multipotente Volwasse Voorloper Sel (MVVS) terapie op genoomwye transkripsie in die twee-week oue rot brein. Die rotte het kort na die perinatale besering die terapie ontvang. MVVS'e is 'n allogeniese stamselproduk, wat belowende effekte in knaagdiermodelle van breinbesering het, maar hul meganisme van werking is meestal onbekend. Ek het gewys dat molekulêre stelsels betrokke by DNA-herstel, sellulêre proliferasie en sinapsherorganisasie deel vorm van die terapeutiese effek. Die toedieningsroete van die MVVS het ' $n$ invloed op die brein transkripsieprofiel 2 weke na toediening gehad.

In hoofstuk 9 het ek bepaal of die MVVS-terapie funksionele beperkings in adolessente en volwasse rotte met PA kan voorkom. Die terapie kognitiewe beperkings gedeeltelik herstel tot in volwassenheid en hiperlokomosie voorkom 
in volwasse manlike rotte, maar dit het nie emosionele steurnisse voorkom nie.

In hoofstuk 10 sluit ek af met 'n algemene bespreking. In hierdie proefskrif het ek getoon dat vroeë-lewe beserings, soos PA, 'n langdurige effek op motoriese beperkings later in lewe het. Hierdie beserings is ' $n$ belangrike risikofaktor vir motoriese steurnisse in die algemene bevolking en in persone met genetiese risikofaktore vir bewegingssteurnisse. Die beperking van die langtermyn skadelike gevolge van PA is noodsaaklik om die ongeskiktheidslas van bewegingssteurnisse dwarsdeur die lewe te verminder. Ons het voordelige effekte getoon van 'n terapie teen eksitotoksisiteit en stamselterapie wat op verskeie molekulêre teikens gerig is. Hierdie terapieë kan verder preklinies vir PA ontwikkel word. 



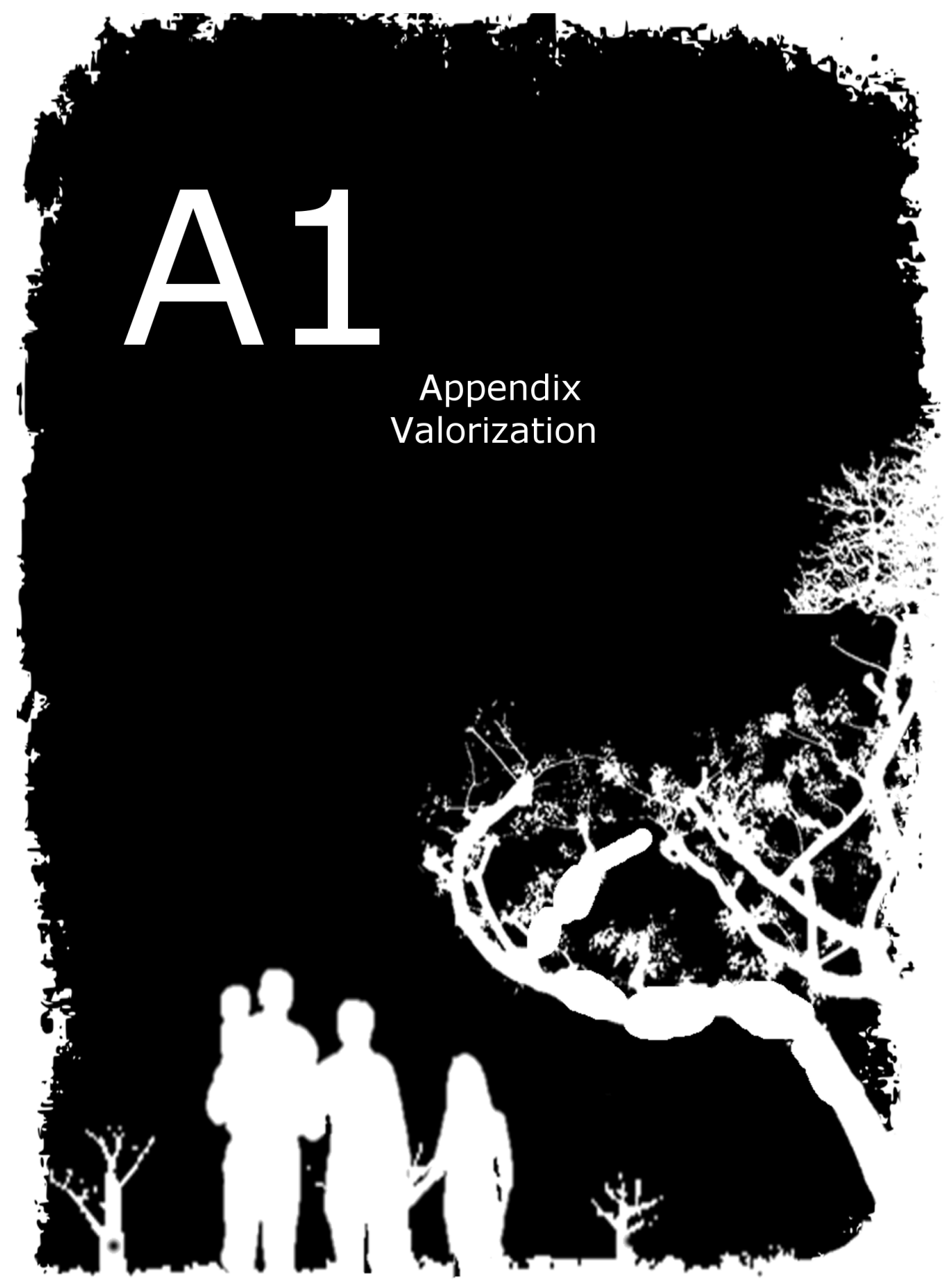




\section{Valorization}

Neurodegenerative diseases, such as Parkinson's disease (PD) and Huntington's disease (HD) are debilitating, progressive disorders that result in a significant reduction in quality of life and substantial healthcare costs for affected individuals. $\mathrm{PD}$ is the $2^{\text {nd }}$ most common neurodegenerative disease, with a rising prevalence according to age. In persons between 40-49 years, PD occurs in 41 cases per 100 000. The incidence drastically increases up to 1903 cases per 100000 persons over 80 (Pringsheim, Jette et al. 2014). This number is expected to increase (Dorsey, Constantinescu et al. 2007). HD is a rare disorder with variable prevalence among geographic locations, but it has a substantial direct economic burden. In Western Europe, HD has an average prevalence of 3.6 cases per 100000 (Squitieri, Griguoli et al. 2015, Rawlins, Wexler et al. 2016). Individuals who may be at risk for these diseases were the target group for the first part of this thesis, as we focused on generating information that can be utilized during genetic counselling for both diseases.

Recently, there has been a drive to identify individuals years before they develop clinical disease to find a population in which disease-preventing therapies could be tested (Paulsen, Langbehn et al. 2008, Noyce, Mencacci et al. 2014). This has generated an ethical dilemma, especially in HD, as there is currently nothing that can be done to prevent the onset of disease. However, the age-of-onset of HD could be delayed by environmental factors like physical activity, stress levels and diet (Mo, Hannan et al. 2015). Current guidelines for genetic counselling recommend against using predictions of diagnosis age based on CAG-repeat expansion length, in part because no curative or preventive treatments are available, and also because such estimates have large confidence intervals (Paulsen, Langbehn et al. 2008). The uptake of genetic testing for HD has remained under $25 \%$ of at-risk individuals since genetic testing has become available (Tassicker, Teltscher et al. 2009).

Identifying other modifiers of the disease onset which can improve the accuracy of disease-onset predictions is crucial to improve the genetic counselling services available to the HD population and assist the individuals which do wish to know with their career trajectory and future social support needs (Baig, Strong et al. 2016). In chapter 4 of this thesis, we identified perinatal modifiers of the age-of-onset of HD. This has direct applications for counselling patients with HD. We investigated the disease-free survival of individuals at-risk for HD which also had a perinatal insult. The inclusion of at-risk individuals is innovative since the majority of previous age-of-onset studies 
been based only on onset ages for CAG-expanded individuals with manifest disease, leading to possible bias from ignoring age distributions among those who have not developed the illness (Paulsen, Langbehn et al. 2008). We followed the epidemiological observations up with molecular insights of the effect of perinatal insults on the striatum, which is affected in HD. This was reviewed in chapter 5. In chapter 6 we showed that perinatal asphyxia (PA) increases nitrosidative stress in the striatum, which is known to degenerate in HD. This is a possible molecular mechanism for the accelerated disease onset seen in HD patients with perinatal insults.

In $\mathrm{PD}$ there is the additional ethical dilemma that only $5-10 \%$ of cases can be ascribed to a currently known monogenetic cause (Bekris, Mata et al. 2010). In the majority of cases, PD is caused by a combination of genetic risk and environmental factors, which could potentially be modified by lifestyle changes such as exercise (Ahlskog 2011). Identifying individuals at an increased risk could potentially facilitate earlier diagnosis and lifestyle changes to prevent the disease. Mutations in the $G B A$ gene are the most common large-effect risk factor for $\mathrm{PD}$, conferring a $9.1 \%$ chance of developing the disease before 80 years (Alcalay, Dinur et al. 2014). We discussed how GBA mutations contribute to $\mathrm{PD}$ risk in detail in chapter 2. We also highlighted that the activity of the $G B A$ enzyme can be boosted by chaperones and this has had promising results in in vitro and animal models (Barkhuizen, Anderson et al. 2016). These chaperones are good candidates for genotype-specific modifier trials in Parkinson's disease. In chapter 3, we investigated the prevalence and mutation spectrum of $G B A$ mutations in South Africans with Parkinson's disease. This knowledge improves the genetic counselling options for South Africans and opens up the possibilities of finding $G B A$-mutation carriers in South Africa to include in clinical trials. Since currently, the genetic status cannot be changed and perinatal insults are still prevalent, despite improvements in maternal care, it is vital to identify therapeutics to limit the damage after these insults.

Given the importance of perinatal events, we focused on therapeutic strategies for PA in the very low gestational age (VLGA) infant in the remainder of this thesis. Hypoxic-ischemic encephalopathy (HIE) due to PA is a common cause of long-term neurological injury in the period around birth. The prevalence of HIE varied from 160 cases per 100000 live births in high income countries to 1490 cases per 100000 births in Sub-Saharan Africa with an average global incidence of 850 infants per 100000 live births globally. This amounted to 1.15 million neonates in 2010 Africa and South-East Asia (Lee, 
Kozuki et al. 2013). In the same period, there were an estimated 15 million preterm births, before 37 weeks of gestation, worldwide (Blencowe, Cousens et al. 2012). Birth before 37 weeks of gestation occurred in approximately $5-8 \%$ of all pregnancies. Very low gestational age (VLGA) births, before 32 weeks of gestation, occurred in approximately $1 \%$ of singletons and $9 \%$ of twin pregnancies (Schaaf, Mol et al. 2011). With advances in medical care, a large proportion of these infants can survive up to adulthood and old-age (Volpe 2009a). The final outcome of encephalopathy due to an asphyxic insult varies among infants according to the severity and the duration of the insult and on the physiological and maturational status of the neonate. Preterm infants are at a greater risk for long-term complications. Motor and cognitive impairment after perinatal asphyxia results in a considerable economic burden (Rees, Harding et al. 2011, Eunson 2015).

Although therapeutic cooling is routinely used in term infants with PA (Davidson, Wassink et al. 2015), it is not yet approved for preterm infants, and small trials indicate that the risk-benefit ratio of this therapy may be unfavorable in VLGA infants (Walsh, Butler et al. 2015). Thus, there is an unmet need for therapies for these infants. In the review in chapter 5, we assessed the usefulness of a rat model of global PA in the VLGA infant as a screening tool for therapeutic approaches. We investigated the potential of mono-target drugs for PA in the VLGA infant on a molecular level. In chapter 6, we show that therapies which prevent supra-physiological increases in nitric oxide synthesis could potentially still be given within 1 week after the insult. This defined a time-window in which to test NO-directed drugs in future preclinical work. In chapter $\mathbf{7}$, we investigated the potential of antagonizing glutamatergic receptors with anesthetics as a mechanism to protect the basal ganglia.

In chapters $\mathbf{8}$ and $\mathbf{9}$, we expanded our investigations to a biological therapy which have an effect on several molecular processes. We tested the preclinical efficacy of multipotent adult progenitor cells (MAPCs) in the PA rat model. Stem cell products have shown therapeutic promise in the ischemia field due to the restorative properties of these cells. The MAPCs are a commercially viable cell product, which uses cells with a lower immunogenic phenotype and higher proliferative potential than the mesenchymal stem cells (MSCs) (Jacobs, Roobrouck et al. 2013). We provided early biochemical pathways altered by these cells which partially elude the mechanisms of these cells; and we evaluated the long-term functional performance of the treated rats to determine which domains should be monitored in human clinical trials with these cells. 
In conclusion, the research described in this thesis has improved the knowledge on factors which modulate the disease-onset in PD and HD, which could be utilized by genetic counsellors for these diseases. We have also expanded the range of therapies which could be developed further for PA in the VLGA infant. These findings contribute to the race to prevent and cure these disorders and pave the way for a well-designed clinical trial based on a sound biological understanding. 



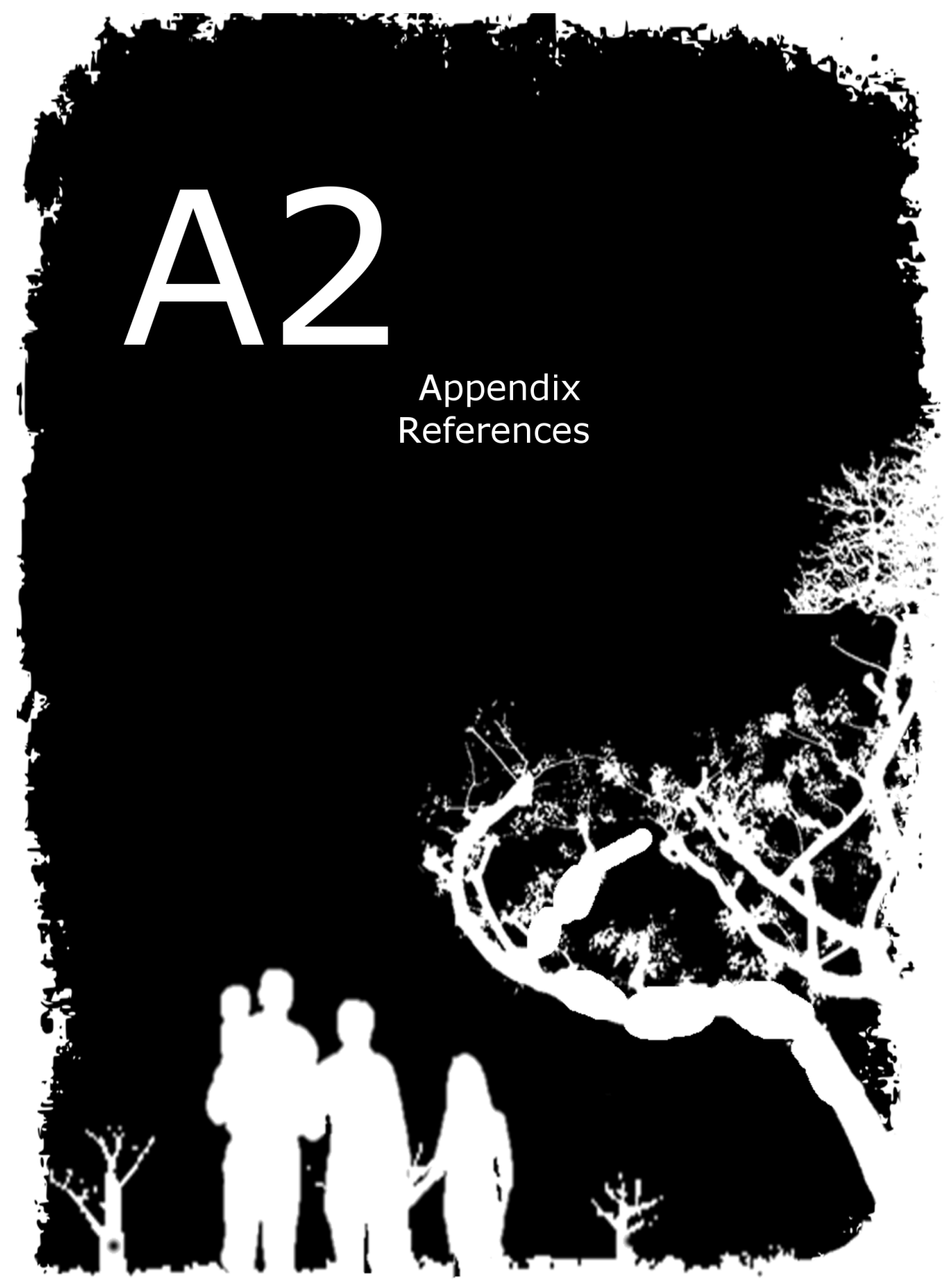




\section{References}

1. ABBOTT, S. K., LI, H., MUÑOZ, S. S., KNOCH, B., BATTERHAM, M., MURPHY, K. E., HALLIDAY, G. M. \& GARNER, B. 2014. Altered ceramide acyl chain length and ceramide synthase gene expression in Parkinson's disease. Movement Disorders, 29, 518-526.

2. ABRAMYCHEVA, N., FEDOTOVA, E., STEPANOVA, M., KLYUSHNIKOV, S., POLESHCHUK, V. \& ILLARIOSHKIN, S. 2014. Extensive screening of GBA gene in Russian patients with Parkinson's disease. Movement Disorders,29,43-44.

3. AFLAKI, E., STUBBlEFIELD, B. K., MANIWANG, E., LOPEZ, G., MOAVEN, N., GOLDIN, E., MARUGAN, J., PATNAIK, S., DUTRA, A. \& SOUTHALL, N. 2014. Macrophage Models of Gaucher Disease for Evaluating Disease Pathogenesis and Candidate Drugs. Science translational medicine, $6,240,73$.

4. AGOSTA, F., KOSTIC, V. S., DAVIDOVIC, K., KRESOJEVIĆ, N., SARRO, L., SVETEL, M., STANKOVIĆ, I., COMI, G., KLEIN, C. \& FILIPPI, M. 2013. White matter abnormalities in Parkinson's disease patients with glucocerebrosidase gene mutations. Movement Disorders, 28, 772-778.

5. AHLSKOG, J. E. 2011. Does vigorous exercise have a neuroprotective effect in Parkinson disease? Neurology, 77, 288-294.

6. AKPAlU, A., RUSCONI, D., ASSElta, R., ADJEI, P., SARFO, F., CHAM, M., AMBONI, M., FABBRI, M., DUGA, S. \& PEZZOLI, G. 2014. Genetics of Parkinson's disease in Ghana: Shall we look for new genes? Movement Disorders, 29, 44.

7. ALCALAY, R., CACCAPPOLO, E., MEJIA-SANTANA, H., TANG, M.-X., ROSADO, L., REILLY, M. O., RUIZ, D., ROSS, B., VERBITSKY, M. \& KISSELEV, S. 2012. Cognitive performance of GBA mutation carriers with early-onset PD The CORE-PD study. Neurology, $78,1434-1440$.

8. ALCALAY, R. N., DINUR, T., QUINN, T., SAKANAKA, K., LEVY, O., WATERS, C., FAHN, S., DOROVSKI, T., CHUNG, W. K. \& PAUCIULO, M. 2014. Comparison of Parkinson Risk in Ashkenazi Jewish Patients With Gaucher Disease and GBA Heterozygotes. JAMA neurology, 71, 752-757.

9. ALCALAY, R. N., LEVY, O. A., WATERS, C. C., FAHN, S., FORD, B., KUO, S.-H., MAZZONI, P., PAUCIULO, M. W., NICHOLS, W. C. \& GAN-OR, Z. 2015. Glucocerebrosidase activity in Parkinson's disease with and without GBA mutations. Brain, 138, 2648-2658.

10. ALFONSO, P., ANDREU, V., PINO-ANGELES, A., GARCÍA-MORENO, M. I., SÁNCHEZJIMÉNEZ, F., POCOVI, M., MELLET, C. O., GARCÍA-FERNÁNDEZ, J. M. \& GIRALDO, P. 2014. Bicyclic derivatives of L-idonojirimycin as pharmacological chaperones for neuronopathic forms of Gaucher disease. Molecular Genetics and Metabolism, 111, S18-S19.

11. ALlENDE-CASTRO, C., ESPINA-MARCHANT, P., BUSTAMANTE, D., ROJAS-MANCILLA, E., NEIRA, T., GUTIERREZ-HERNANDEZ, M., ESMAR, D., VALDES, J., MORALES, P. \& GEBICKE-HAERTER, P. 2012. Further studies on the hypothesis of PARP-1 inhibition as a strategy for lessening the long-term effects produced by perinatal asphyxia: effects of nicotinamide and theophylline on PARP-1 activity in brain and peripheral tissue. Neurotoxicity research, 22, 79-90.

12. ALTINTAŞ, Ö., IŞERI, P., ÖZKAN, B. \& ÇAĞLAR, Y. 2008. Correlation between retinal morphological and functional findings and clinical severity in Parkinson's disease. Documenta Ophthalmologica, 116, 137-146. 
13. ALVAREZ-DIAZ, A., HILARIO, E., GONI DE CERIO, F., VALLS-I-SOLER, A. \& ALVAREZDIAZ, F. 2007. Hypoxic-ischemic injury in the immature brain-key vascular and cellular players. Neonatology, 92, 227-235.

14. ALVAREZ-ERVITI, L., SEOW, Y., SCHAPIRA, A. H., GARDINER, C., SARGENT, I. L., WOOD, M. J. \& COOPER, J. M. 2011. Lysosomal dysfunction increases exosome-mediated alpha-synuclein release and transmission. Neurobiology of disease, 42, 360-367.

15. AmBrosi, G., GHEZZI, C., ZANGAGLIA, R., LEVANDIS, G., PACCHETTI, C. \& BLANDINI, F. 2015. Ambroxol-induced rescue of defective glucocerebrosidase is associated with increased LIMP-2 and saposin C levels in GBA1 mutant Parkinson's disease cells. Neurobiology of Disease.

16. ANDERSSON, K., BLUM, M., CHEN, Y., ENEROTH, P., GROSS, J., HERRERA-MARSCHITZ, M., BJELKE, B., BOLME, P., DIAZ, R. \& JAMISON, L. 1995. Perinatal asphyxia increases bFGF mRNA levels and DA cell body number in the mesencephalon of rats. Neuroreport, 6 , 375-378.

17. ANDRÉ, V. M., CEPEDA, C. \& LEVINE, M. S. 2010. Dopamine and glutamate in Huntington's disease: a balancing act. CNS neuroscience \& therapeutics, 16, 163-178.

18. ANKLESHWARIA, C., SHETH, J., MiSTRI, M., BAVDEKAR, A., NAMPOOTHIRI, S., GUPTA, S. \& SHETH, F. 2014. Identification of novel mutations in glucocerebrosidase (GBA) gene in Indian patients with gaucher disease (GD). Molecular Cytogenetics, 7, P52.

19. Antoniades, C. A., Demeyere, N., Kennard, C., HUMPhreys, G. W. \& HU, M. T. 2015. Antisaccades and executive dysfunction in early drug-naive Parkinson's disease: The discovery study. Movement Disorders, 30, 843-847.

20. ARANCIO, O. \& CHAO, M. V. 2007. Neurotrophins, synaptic plasticity and dementia. Current opinion in neurobiology, 17, 325-330.

21. ARChibald, N., ClARKE, M., MOSImAnN, U. \& BURN, D. 2011. Retinal thickness in Parkinson's disease. Parkinsonism \& related disorders, 17, 431-436.

22. ARMSTRONG-WELLS, J., BERNARD, T. J., BOADA, R. \& MANCO-JOHNSON, M. 2010. Neurocognitive outcomes following neonatal encephalopathy. NeuroRehabilitation, 26, 27-33.

23. ARCHER, D.P., WALKER, A.M., MCCANN, S.K., MOSER, J.J., APPIREDDY, R.M. 2017.Anesthetic Neuroprotection in Experimental Stroke in Rodents. A Systematic Review and Meta-analysis. The Journal of the American Society of Anesthesiologists 126, 653-665.

24. ARNDT, S., HEITNER, R., LANE, A. \& RAMSAY, M. 2009. Glucocerebrosidase gene mutations in black South Africans with Gaucher disease. Blood Cells, Molecules, and Diseases, $43,129-133$

25. ARTEAGA-BRACHO, E. E., GULINELLO, M., WINCHESTER, M. L., PICHAMOORTHY, N., PETRONGlO, J. R., ZAMBRANO, A. D., INOCENCIO, J., DE JESUS, C. D., LOUIE, J. O. \& GOKHAN, S. 2016. Postnatal and adult consequences of loss of huntingtin during development: Implications for Huntington's disease. Neurobiology of Disease, 96, 144-155.

26. ASHDOWN, H., JOITA, S., LUHESHI, G. N. \& BOKSA, P. 2008. Acute brain cytokine responses after global birth hypoxia in the rat. Journal of neuroscience research, 86, 34013409 .

27. ASSElta, R., RIMOldi, V., SIRI, C., CILIA, R., GUella, I., TESEI, S., SOldÀ, G., PEZZOLI, G., DUGA, S. \& GOLDWURM, S. 2014. Glucocerebrosidase mutations in primary parkinsonism. Parkinsonism \& related disorders, 20, 1215-1220. 
28. AWAD, O., SARKAR, C., PANICKER, L. M., MILLER, D., ZENG, X., SGAMBATO, J. A., LIPINSKI, M. M. \& FELDMAN, R. A. 2015. Altered TFEB-mediated lysosomal biogenesis in Gaucher disease iPSCs-derived neuronal cells. Human Molecular Genetics,24, 5775-5788.

29. AZZOPARDI, D., STROHM, B., MARLOW, N., BROCKLEHURST, P., DEIERL, A., EDDAMA, O., GOODWIN, J., HALLIDAY, H. L., JUSZCZAK, E. \& KAPELLOU, O. 2014. Effects of hypothermia for perinatal asphyxia on childhood outcomes. New England Journal of Medicine, 371, 140-149.

30. BACK, S. A. 2017. White matter injury in the preterm infant: pathology and mechanisms. Acta Neuropathologica, 1-19.

31. BAE, E.-J., YANG, N.-Y., SONG, M., LEE, C. S., LEE, J. S., JUNG, B. C., LEE, H.-J., KIM, S., MASLIAH, E. \& SARDI, S. P. 2014. Glucocerebrosidase depletion enhances cell-to-cell transmission of $\alpha$-synuclein. Nature communications, 5,4755

32. BAE, E.-J., YANG, N. Y., LEE, C., LEE, H.-J., KIM, S., SARDI, S. P. \& LEE, S.-J. 2015. Loss of glucocerebrosidase 1 activity causes lysosomal dysfunction and $\alpha$-synuclein aggregation. Experimental \& molecular medicine, 47, e153.

33. BAIG, S. S., STRONG, M., ROSSER, E., TAVERNER, N. V., GLEW, R., MIEDZYBRODZKA, Z., ClARKE, A., CRAUFURD, D. \& QUARRELL, O. W. 2016. 22 Years of predictive testing for Huntington's disease: the experience of the UK Huntington's Prediction Consortium. European Journal of Human Genetics, 24, 1396-1402.

34. BAlleine, B. W., DELGADO, M. R. \& HIKOSAKA, O. 2007. The role of the dorsal striatum in reward and decision-making. The Journal of Neuroscience, 27, 8161-8165.

35. BALWANI, M., FUERSTMAN, L., KORNREICH, R., EDELMANN, L. \& DESNICK, R. J. 2010. Type 1 Gaucher disease: significant disease manifestations in "asymptomatic" homozygotes. Archives of internal medicine, 170, 1463-1469.

36. BARAN, H., KEPPLINGER, B., HERRERA-MARSCHITZ, M., STOLZE, K., LUBEC, G. \& NOHL, H. 2001. Increased kynurenic acid in the brain after neonatal asphyxia. Life sciences, $69,1249-1256$.

37. BARDIEN, S., KEYSER, R., YAKO, Y., LOMBARD, D. \& CARR, J. 2009. Molecular analysis of the parkin gene in South African patients diagnosed with Parkinson's disease. Parkinsonism \& related disorders, 15, 116-121.

38. BARDIEN, S., MARSBERG, A., KEYSER, R., LOMBARD, D., LESAGE, S., BRICE, A. \& CARR, J. 2010. LRRK2 G2019S mutation: frequency and haplotype data in South African Parkinson's disease patients. Journal of neural transmission, 117, 847-853.

39. BARKhUizEn, M., ANDERSON, D. G. \& GROBLER, A. F. 2016a. Advances in GBAassociated Parkinson's disease-Pathology, presentation and therapies. Neurochemistry international, 93, 6-25.

40. BARKHUIZEN, M., ANDERSON, D. G., VAN DER WESTHUIZEN, F. H. \& GROBLER, A. F. 2016b. "Benign" GBA variants may alter gene splicing in South African Parkinson's disease cases. Parkinsonism \& Related Disorders, 22, e172.

41. BARKHUIZEN, M., VAN DE BERG, W. D., DE VENTE, J., BLANCO CALVO, E., GAVILANES, A. W. \& STEINBUSCH, H. W. M. 2017a. Nitric Oxide Production in the Striatum and Cerebellum of a Rat Model of Preterm Global Perinatal Asphyxia. Neurotoxicity research, 31, 400-409.

42. BARKhUizen, M., VAN DEN HOVE, D., VLES, J., STEINBUSCH, H., KRAMER, B. \& GAVILANES, A. 2017b. 25 years of research on global asphyxia in the immature rat brain. Neuroscience \& Biobehavioral Reviews. 
43. BARNAT, M., LE FRIEC, J., BENSTAALI, C. \& HUMBERT, S. 2017. Huntingtin-mediated multipolar-bipolar transition of newborn cortical neurons is critical for their postnatal neuronal morphology. Neuron, 93, 99-114.

44. BARRETT, M., HAGENAH, J., DHAWAN, V., PENG, S., STANLEY, K., RAYMOND, D., DEIK, A., GROSS, S., SCHREIBER-AGUS, N. \& MIRELMAN, A. 2013. Transcranial sonography and functional imaging in glucocerebrosidase mutation Parkinson disease. Parkinsonism \& related disorders, 19, 186-191.

45. BARRETT, M., SHANKER, V., SEVERT, W., RAYMOND, D., GROSS, S., SCHREIBERAGUS, N., KORNREICH, R., OZELIUS, L., BRESSMAN, S. \& SAUNDERS-PULLMAN, R. 2014. Cognitive and Antipsychotic Medication Use in Monoallelic GBA-Related Parkinson Disease. JIMD Reports, 16, 31-38.

46. Battaglia, S., MAguire, O., Thorne, J. L., HORNung, L. B., DOIG, C. L., LiU, S., SUCHESTON, L. E., BIANCHI, A., KHANIM, F. L. \& GOMMERSALL, L. M. 2010. Elevated NCOR1 disrupts PPAR $\alpha \gamma$ signaling in prostate cancer and forms a targetable epigenetic lesion. Carcinogenesis, 31, 1650-1660.

47. BEAVAN, M., MCNEILL, A., PROUKAKIS, C., HUGHES, D. A., MEHTA, A. \& SCHAPIRA, A. 2015. Evolution of Prodromal Clinical Markers of Parkinson Disease in a GBA Mutation-Positive Cohort. JAMA neurology, 72, 201-208.

48. BEDI, S. S., HETZ, R., THOMAS, C., SMITh, P., OLSEN, A. B., WILliAMS, S., XUE, H., AROOM, K., URAY, K. \& HAMILTON, J. 2013. Intravenous multipotent adult progenitor cell therapy attenuates activated microglial/macrophage response and improves spatial learning after traumatic brain injury. Stem Cells Transl Med, 2, 953-960.

49. BEKIESINSKA-FIGATOWSKA, M., MIERZEWSKA, H. \& JURKIEWICZ, E. 2013. Basal ganglia lesions in children and adults. European journal of radiology, 82, 837-849.

50. BEKRIS, L. M., MATA, I. F. \& ZABETIAN, C. P. 2010. The genetics of Parkinson disease. Journal of geriatric psychiatry and neurology, 23, 228-242.

51. BENDIKOV-BAR, I., MAOR, G., FILOCAMO, M. \& HOROWITZ, M. 2013. Ambroxol as a pharmacological chaperone for mutant glucocerebrosidase. Blood Cells, Molecules, and Diseases, 50, 141-145.

52. BENDIKOV-BAR, I., RAPAPORT, D., LARISCH, S. \& HOROWITZ, M. 2014. Parkinmediated ubiquitination of mutant glucocerebrosidase leads to competition with its substrates PARIS and ARTS. Orphanet journal of rare diseases, 9, 86 .

53. BENDIKOV-BAR, I., RON, I., FILOCAMO, M. \& HOROWITZ, M. 2011. Characterization of the ERAD process of the L444P mutant glucocerebrosidase variant. Blood Cells, Molecules, and Diseases, 46, 4-10.

54. BENiteZ, B. A., DAVIS, A. A., JIN, S. C., IBANEZ, L., ORTEGA-CUBERO, S., PASTOR, P., CHOI, J., COOPER, B., PERLMUTTER, J. S. \& CRUCHAGA, C. 2016. Resequencing analysis of five Mendelian genes and the top genes from genome-wide association studies in Parkinson's Disease. Molecular Neurodegeneration, 11, 1.

55. BENKO, W., RIES, M., WIGGS, E. A., BRADY, R. O., SCHIFFMANN, R. \& FITZGIBBON, E. J. 2011. The saccadic and neurological deficits in type 3 Gaucher disease. PLoS One, 6, e22410.

56. BENNET, L., ROELFSEMA, V., PATHIPATI, P., QUAEDACKERS, J. \& GUNN, A. 2006 Relationship between evolving epileptiform activity and delayed loss of mitochondrial activity after asphyxia measured by near-infrared spectroscopy in preterm fetal sheep. The Journal of physiology, 572, 141-154. 
57. BERGER, N., VAILLANCOURT, C. \& BOKSA, P. 2000. Interactive effects of anoxia and general anesthesia during birth on the degree of CNS and systemic hypoxia produced in neonatal rats. Experimental brain research, 131, 524-531.

58. BeRnert, G., HOEger, H., MOSGOELlER, W., STOLZLECHNER, D. \& LUBEC, B. 2003. Neurodegeneration, neuronal loss, and neurotransmitter changes in the adult guinea pig with perinatal asphyxia. Pediatric research, 54, 523-528.

59. BetTencourt, C., Hensman-Moss, D., FLOWER, M., WIETHOFF, S., BRICE, A., GOIZET, C., STEVANIN, G., KOUTSIS, G., KARADIMA, G. \& PANAS, M. 2016. DNA repair pathways underlie a common genetic mechanism modulating onset in polyglutamine diseases. Annals of neurology, 79, 983-990.

60. BINDEA, G., MLECNIK, B., HACKL, H., CHAROENTONG, P., TOSOlini, M., KIRILOVSKY, A., FRIDMAN, W.-H., PAGÈS, F., TRAJANOSKI, Z. \& GALON, J. 2009. ClueGO: a Cytoscape plug-in to decipher functionally grouped gene ontology and pathway annotation networks. Bioinformatics, 25, 1091-1093.

61. BINIENDA, Z., HOLSON, R., CHEN, F.-X., ORIAKU, E., KIM, C., FLYNN, T., SLIKKER, W., PAULE, M., FEUERS, R. \& FERGUSON, S. 1996. Effects of ischemia-hypoxia induced by interruption of uterine blood flow on fetal rat liver and brain enzyme activities and offspring behavior. International journal of developmental neuroscience, 14, 399-408.

62. BJELKE, B., ANDERSSON, K., ÖGREN, S. O. \& BOLME, P. 1991. Asphyctic lesion: proliferation of tyrosine hydroxylase-immunoreactive nerve cell bodies in the rat substantia nigra and functional changes in dopamine neurotransmission. Brain research, 543, 1-9.

63. BLACK, R. E., COUSENS, S., JOHNSON, H. L., LAWN, J. E., RUDAN, I., BASSANI, D. G., JHA, P., CAMPBELL, H., WALKER, C. F. \& CIBULSKIS, R. 2010. Global, regional, and national causes of child mortality in 2008: a systematic analysis. The Lancet, 375, 1969-1987.

64. BLACK, S. M., BEDOLLI, M. A., MARTINEZ, S., BRISTOW, J. D., FERRIERO, D. M. \& SOIFER, S. J. 1995. Expression of neuronal nitric oxide synthase corresponds to regions of selective vulnerability to hypoxia-ischaemia in the developing rat brain. Neurobiology of disease, 2, 145-155.

65. BLANCKENBERG, J., NTSAPI, C., CARR, J. A. \& BARDIEN, S. 2014. EIF4G1 R1205H and VPS35 D620N mutations are rare in Parkinson's disease from South Africa. Neurobiology of aging, 35, 445. e3.

66. Blanco, E., GAleANO, P., HOLUBieC, M. I., ROMERO, J. I., LOGICA, T., RIVERA, P., PAVÓN, F. J., SUAREZ, J., CAPANI, F. \& DE FONSECA, F. R. 2015. Perinatal asphyxia results in altered expression of the hippocampal acylethanolamide/endocannabinoid signaling system associated to memory impairments in postweaned rats. Frontiers in neuroanatomy, 9, 141.

67. BLANCO, S., MOLINA, F. J., CASTRO, L., DEL MORAL, M. L., HERNANDEZ, R., JIMENEZ, A., RUS, A., MARTINEZ-LARA, E., SILES, E. \& PEINADO, M. A. 2010. Study of the nitric oxide system in the rat cerebellum during aging. BMC neuroscience, 11, 78.

68. Blencowe, H., COUSEns, S., CHOU, D., OestergaARD, M., SAY, L., MOLleR, A.-B., KINNEY, M. \& LAWN, J. 2013. Born too soon: the global epidemiology of 15 million preterm births. Reproductive health, 10, 1.

69. Blencowe, H., COUSEns, S., OestergaARD, M. Z., CHOU, D., MOLlER, A.-B. NARWAL, R., ADLER, A., GARCIA, C. V., ROHDE, S. \& SAY, L. 2012. National, regional, and worldwide estimates of preterm birth rates in the year 2010 with time trends since 1990 for selected countries: a systematic analysis and implications. The Lancet, 379, 2162-2172. 
70. BOKSA, P. 1998. Birth insult alters ethanol preference in the adult rat. European journal of pharmacology, 348, 143-153.

71. BOKSA, P. \& EL-KHODOR, B. 2003. Birth insult interacts with stress at adulthood to alter dopaminergic function in animal models: possible implications for schizophrenia and other disorders. Neuroscience \& Biobehavioral Reviews, 27, 91-101.

72. BOKSA, P., KRISHNAMURTHY, A. \& BROOKS, W. 1995. Effects of a period of asphyxia during birth on spatial learning in the rat. Pediatric Research, 37, 489-96.

73. BOKSA, P. \& ZHANG, Y. 2008. Global birth hypoxia increases the neonatal testosterone surge in the rat. Neuroendocrinology, 88, 266-275.

74. BOKSA, P., ZHANG, Y., AMRITRAJ, A. \& KAR, S. 2006. Birth insults involving hypoxia produce long-term increases in hippocampal [125 I] insulin-like growth factor-I and-II receptor binding in the rat. Neuroscience, 139, 451-462.

75. BONSIGNORE, L. T., VENEROSI, A., CHIAROTTI, F., ALLEVA, E. \& CIRULli, F. 2006 Acute perinatal asphyxia at birth has long-term effects on behavioural arousal and maternal behaviour in lactating rats. Behavioural brain research, 172, 54-62.

76. BOOT, B. P., BOEVE, B. F., ROBERTS, R. O., FERMAN, T. J., GEDA, Y. E., PANKRATZ, V. S., IVNIK, R. J., SMITH, G. E., MCDADE, E. \& CHRISTIANSON, H. 2012. Probable rapid eye movement sleep behavior disorder increases risk for mild cognitive impairment and Parkinson disease: A population-based study. Annals of neurology, 71, 49-56.

77. BÖtTCher, T., ROLFS, A., MEYeR, B., GROSSMANN, A., BERG, D., KROPP, P., BENECKE, R. \& WALTER, U. 2013. Clinical, genetic, and brain sonographic features related to Parkinson's disease in Gaucher disease. Journal of neurology, 260, 2523-2531.

78. BRAKE, W., NOEL, M., BOKSA, P. \& GRATTON, A. 1997a. Influence of perinatal factors on the nucleus accumbens dopamine response to repeated stress during adulthood: an electrochemical study in the rat. Neuroscience, 77, 1067-1076.

79. BRAKE, W. G., BOKSA, P. \& GRATTON, A. 1997b. Effects of perinatal anoxia on the acute locomotor response to repeated amphetamine administration in adult rats. Psychopharmacology, 133, 389-395.

80. BRAKE, W. G., SUlLivAN, R. M. \& GRATTON, A. 2000. Perinatal distress leads to lateralized medial prefrontal cortical dopamine hypofunction in adult rats. The Journal of Neuroscience, 20, 5538-5543.

81. BRAMBLETT, G. T., GOEDERT, M., JAKES, R., MERRICK, S. E., TROJANOWSKI, J. Q. \& LEE, V. M. 1993. Abnormal tau phosphorylation at Ser 396 in Alzheimer's disease recapitulates development and contributes to reduced microtubule binding. Neuron, 10, 10891099

82. BRISCH, R., SANIOTIS, A., WOLF, R., BIELAU, H., BERNSTEIN, H.-G., STEINER, J., BOGERTS, B., BRAUN, K., JANKOWSKI, Z. \& KUMARATILAKE, J. 2014. The role of dopamine in schizophrenia from a neurobiological and evolutionary perspective: old fashioned, but still in vogue. Frontiers in psychiatry, 5, 47.

83. BROCKMANN, K., HILKER, R., PILATUS, U., BAUDREXEL, S., SRULIJES, K., MAGERKURTH, J., HAUSER, A.-K., SCHUlte, C., CSOTI, I. \& MERTEN, C. D. 2012. GBA-associated PD Neurodegeneration, altered membrane metabolism, and lack of energy failure. Neurology, $79,213-220$. 
84. Brockmann, K., SRUliJes, K., PFlederer, S., HAUSER, A. K., SCHulte, C., MAETZLER, W., GASSER, T. \& BERG, D. 2015. GBA-associated Parkinson's disease: Reduced survival and more rapid progression in a prospective longitudinal study. Movement Disorders, 30, 407-411.

85. BRYAN, N. S. \& GRISHAM, M. B. 2007. Methods to detect nitric oxide and its metabolites in biological samples. Free Radical Biology and Medicine, 43, 645-657.

86. BUCKMASTER, P. S. 2014. Does mossy fiber sprouting give rise to the epileptic state? Issues in Clinical Epileptology: A View from the Bench. Springer.

87. BUONOCORE, G., PERRONE, S., TURRISI, G., W KRAMER, B. \& BALDUINI, W. 2012 New pharmacological approaches in infants with hypoxic-ischemic encephalopathy. Current pharmaceutical design, 18, 3086-3100.

88. BURKE, D. G., RAHIM, A. A., WADDINGTON, S. N., KARLSSON, S., ENQUIST, I., BHATIA, K., MEHTA, A., VELLODI, A. \& HEALES, S. 2013. Increased glucocerebrosidase (GBA) 2 activity in GBA1 deficient mice brains and in Gaucher leucocytes. Journal of inherited metabolic disease, 36, 869-872.

89. BURKE, R., MACAYA, A., DEVIVO, D., KENYON, N. \& JANEC, E. 1992. Neonatal hypoxic-ischemic or excitotoxic striatal injury results in a decreased adult number of substantia nigra neurons. Neuroscience, 50, 559-569.

90. BUSTAMANTE, D., GOINY, M., ÅSTRÖM, G., GROSS, J., ANDERSSON, K. \& HERRERAMARSCHITZ, M. 2003. Nicotinamide prevents the long-term effects of perinatal asphyxia on basal ganglia monoamine systems in the rat. Experimental brain research, 148, 227-232.

91. BUSTAMANTE, D., MORAles, P., PEREYRA, J. T., GOINY, M. \& HERRERA-MARSCHITZ, M. 2007. Nicotinamide prevents the effect of perinatal asphyxia on dopamine release evaluated with in vivo microdialysis 3 months after birth. Experimental brain research, 177, 358-369.

92. CABAJ, A., BEKIESIŃSKA-FIGATOWSKA, M. \& MADZIK, J. 2012. MRI patterns of hypoxic-ischemic brain injury in preterm and full term infants-classical and less common MR findings. Pol J Radiol, 77, 71-76.

93. CAlamandrei, G., Venerosi, A. P., VAlanzano, A., DE BERARDinis, M. A., GRECO, A., PUOPOLO, M. \& MINGHETTI, L. 2004. Increased brain levels of F2-isoprostane are an early marker of behavioral sequels in a rat model of global perinatal asphyxia. Pediatric research, 55, 85-92.

94. CAMERON, S. H., ALWAKEEL, A. J., GODDARD, L., HOBBS, C. E., GOWING, E. K., BARNETT, E. R., KOHE, S. E., SIZEMORE, R. J. \& OORSCHOT, D. E. 2015 . Delayed post-treatment with bone marrow-derived mesenchymal stem cells is neurorestorative of striatal medium-spiny projection neurons and improves motor function after neonatal rat hypoxia-ischemia. Molecular and Cellular Neuroscience, 68, 56-72.

95. CAnZOniero, L. M., GRAnZOtto, A., Turetsky, D. M., ChOI, D. W., DUGAN, L. L. \& SENSI, S. L. 2014. nNOS (+) striatal neurons, a subpopulation spared in Huntington's Disease, possess functional NMDA receptors but fail to generate mitochondrial ROS in response to an excitotoxic challenge. Mitochondria: the cell powerhouse and nexus of stress, 64.

96. CAPANI, F., LOIDL, C. F., AGUIRRe, F., PIEHL, L., FACORRO, G., HAGER, A., DE PAOLI, T., FARACH, H. \& PECCI-SAAVEDRA, J. 2001. Changes in reactive oxygen species (ROS) production in rat brain during global perinatal asphyxia: an ESR study. Brain Research, 914, 204-7. 
97. CAPAni, F., LOIDL, C. F., PIEHL, L. L., FACORRO, G., DE PAOli, T. \& HAGER, A. 2003. Long term production of reactive oxygen species during perinatal asphyxia in the rat central nervous system: effects of hypothermia. International journal of neuroscience, 113, 641-654.

98. CAPANI, F., LOIDL, F., LOPEZ-COSTA, J. J., SELVIN-TESTA, A. \& SAAVEDRA, J. P. 1997. Ultrastructural changes in nitric oxide synthase immunoreactivity in the brain of rats subjected to perinatal asphyxia: neuroprotective effects of cold treatment. Brain Research, $775,11-23$.

99. CAPANi, F., SARACENO, G. E., BOtTI, V., AON-BERTOLINO, L., DE OLIVEIRA, D. M., BARreto, G., GAlEANO, P., GIRAldeZ-AlVAREZ, L. D. \& COIRINI, H. 2009 Protein ubiquitination in postsynaptic densities after hypoxia in rat neostriatum is blocked by hypothermia. Experimental neurology, 219, 404-413.

100. CARBAJO-PESCADOR, S., ORDONEZ, R., BENET, M., JOVER, R., GARCÍA-PALOMO, A., MAURIZ, J. \& GONZÁLEZ-GALLEGO, J. 2013. Inhibition of VEGF expression through blockade of Hif1 $\alpha$ and STAT3 signalling mediates the anti-angiogenic effect of melatonin in HepG2 liver cancer cells. British journal of cancer, 109, 83.

101. CARniglia, L., RAmíRez, D., DURAnd, D., SABA, J., TURATi, J., CARUso, C., SCIMONELLI, T. N. \& LASAGA, M. 2017. Neuropeptides and microglial activation in inflammation, pain, and neurodegenerative diseases. Mediators of inflammation, 2017.

102. CASSINERIO, E., GRAZIADEI, G. \& POGGIALI, E. 2014. Gaucher disease: a diagnostic challenge for internists. European journal of internal medicine, 25, 117-124.

103. CEBRAL, E., CAPANI, F., SELVIN-TESTA, A., FUNES, M. R., COIRINI, H. \& LOIDL, C. F. 2006. Neostriatal cytoskeleton changes following perinatal asphyxia: effect of hypothermia treatment. International journal of neuroscience, 116, 697-714.

104. CEBRAL, E. \& LOIDL, C. F. 2011. Changes in neostriatal and hippocampal synaptic densities in perinatal asphyctic male and female young rats: Role of hypothermia. Brain research bulletin, 84, 31-38.

105. CERESOLI-BORRONI, G. \& SCHWARCZ, R. 2000. Perinatal kynurenine pathway metabolism in the normal and asphyctic rat brain. Amino Acids, 19, 311-323.

106. CERESOLI-BORRONI, G. \& SCHWARCZ, R. 2001. Neonatal asphyxia in rats: acute effects on cerebral kynurenine metabolism. Pediatric research, 50, 231-235.

107. CERESOli-BORRONI, G., GUIDETTI, P., AMORI, L., PELliCCIARI, R. \& SCHWARCZ, R. 2007. Perinatal kynurenine 3-hydroxylase inhibition in rodents: Pathophysiological implications. Journal of neuroscience research, 85, 845-854.

108. CHAHINE, L. M., QIANG, J., ASHBRIDGE, E., MINGER, J., YEAROUT, D., HORN, S. COLCHER, A., HURTIG, H. I., LEE, V. M. \& VAN DEERLIN, V. M. 2013. Clinical and biochemical differences in patients having Parkinson disease with vs without GBA mutations. JAMA neurology, 70, 852-858

109. CHARRIAUt-MARLANGUE, C., BONNIN, P., GHARIB, A., LEGER, P.-L., VILlAPOL, S., POCARD, M., GRESSENS, P., RENOLlEAU, S. \& BAUD, O. 2012. Inhaled nitric oxide reduces brain damage by collateral recruitment in a neonatal stroke model. Stroke, 43 , 3078-3084.

110. CHATTOPADHYAYA, B. \& DI CRISTO, G. 2012. GABAergic circuit dysfunctions in neurodevelopmental disorders. Frontiers in psychiatry, 3, 51.

111. CHAUDHURI, K., HEALY, D. G. \& SCHAPIRA, A. H. 2006. Non-motor symptoms of Parkinson's disease: diagnosis and management. The Lancet Neurology, 5, 235-245. 
112. ChaVes, R. G., PEREIRA, L., De ARAÚJo, F., ROZEnBERG, R., CARVAlHO, M., COELHO, J., MICHELIN-TIRELLI, K., CHAVES, M. \& CAVALCANTI, G. 2014. Consanguinity and founder effect for Gaucher disease mutation G377S in a population from Tabuleiro do Norte, Northeastern Brazil. Clinical genetics, 88, 391-395.

113. Chen, Y., BJELKE, B., BOLME, P., ENEROTH, P., GROSS, J., LOIDL, F., HERRERAMARSCHITZ, M. \& ANDERSSN, K. 1995. Nicotine treatment counteracts perinatal asphyxiainduced changes in the mesostriatal/limbic dopamine systems and in motor behaviour in the four-week-old male rat. Neuroscience, 68, 531-538.

114. CHEN, Y., ENGIDAWORK, E., LOIDL, F., DELL'ANNA, E., GOINY, M., LUBEC, G. ANDERSSON, K. \& HERRERA-MARSCHITZ, M. 1997a. Short-and long-term effects of perinatal asphyxia on monoamine, amino acid and glycolysis product levels measured in the basal ganglia of the rat. Developmental brain research, 104, 19-30.

115. CHEN, Y., HiLleForS-BERGLUND, M., HERRERA-MARSCHITZ, M., BJELKE, B., GROSS, J., ANDERSSON, K. \& VON EULER, G. 1997b. Perinatal asphyxia induces longterm changes in dopamine D 1, D 2, and D 3 receptor binding in the rat brain. Experimental neurology, 146, 74-80.

116. CHIASSERINI, D., PACIOTTI, S., EUSEBI, P., PERSICHETTI, E., TASEGIAN, A., KURZAWAAKANBI, M., CHINNERY, P. F., MORRIS, C. M., CALABRESI, P. \& PARNETTI, L. 2015. Selective loss of glucocerebrosidase activity in sporadic Parkinson's disease and dementia with Lewy bodies. Molecular Neurodegeneration, 10, 15.

117. CHU, Y., DODIYA, H., AEBISCHER, P., OLANOW, C. W. \& KORDOWER, J. H. 2009. Alterations in lysosomal and proteasomal markers in Parkinson's disease: relationship to alpha-synuclein inclusions. Neurobiology of disease, 35, 385-398.

118. CHUN, P. T., MCPHERSON, R. J., MARNEY, L. C., ZANGENEH, S. Z., PARSONS, B. A., SHOJAIE, A., SYNOVEC, R. E. \& JUUL, S. E. 2015. Serial plasma metabolites following hypoxic-ischemic encephalopathy in a nonhuman primate model. Developmental neuroscience, 37, 161-171.

119. CIRULli, F., BONSIGNORE, L., VENEROSI, A., VALANZANO, A., CHIAROTTI, F. \& ALLEVA, E. 2003. Long-term effects of acute perinatal asphyxia on rat maternal behavior. Neurotoxicology and teratology, 25, 571-578.

120. CLARK, L., ROSS, B., WANG, Y., MEJIA-SANTANA, H., HARRIS, J., LOUIS, E., COTE, L., ANDREWS, H., FAHN, S. \& WATERS, C. 2007. Mutations in the glucocerebrosidase gene are associated with early-onset Parkinson disease. Neurology, 69, 1270-1277.

121. CLARK, L. N., CHAN, R., CHENG, R., LIU, X., PARK, N., PARMALEE, N., KISSELEV, S., CORTES, E., TORRES, P. A. \& PASTORES, G. M. 2015. Gene-Wise Association of Variants in Four Lysosomal Storage Disorder Genes in Neuropathologically Confirmed Lewy Body Disease. PloS one, 10, p.e0125204

122. ClARK, L. N., KARTSAKLIS, L. A., GILBERT, R. W., DORADO, B., ROSS, B. M., KISSELEV, S., VERBITSKY, M., MEJIA-SANTANA, H., COTE, L. J. \& ANDREWS, H. 2009. Association of glucocerebrosidase mutations with dementia with lewy bodies. Archives of neurology, 66, 578-583.

123. CLARK, L. N., KISSELEV, S., PARK, N., ROSS, B., VERBITSKY, M., RIOS, E., ALCALAY, R. N., LEE, J. H. \& LOUIS, E. D. 2010. Mutations in the Parkinson's disease genes, Leucine Rich Repeat Kinase 2 (LRRK2) and Glucocerebrosidase (GBA), are not associated with essential tremor. Parkinsonism \& related disorders, 16, 132-135. 
124. COMPTA, Y., PARKKINEN, L., KEMPSTER, P., SELIKHOVA, M., LASHLEY, T., HOLTON, J. L., LEES, A. J. \& REVESZ, T. 2014. The significance of $\alpha$-synuclein, amyloid- $\beta$ and tau pathologies in Parkinson's disease progression and related dementia. Neurodegenerative Diseases, 13, 154-156.

125. CONTESTABILE, A. 2012. Role of nitric oxide in cerebellar development and function: focus on granule neurons. The Cerebellum, 11, 50-61.

126. CORTI, O., LESAGE, S. \& BRICE, A. 2011. What genetics tells us about the causes and mechanisms of Parkinson's disease. Physiological reviews, 91, 1161-1218.

127. COUNSELL, S. J., TRANTER, S. L. \& RUTHERFORD, M. A. Magnetic resonance imaging of brain injury in the high-risk term infant. Seminars in perinatology, 2010. Elsevier, 67-78

128. COX-LIMPENS, K. E., VLES, J. S., VAN DEN HOVE, D. L., ZIMMERMANN, L. J. \& GAVILANES, A. W. 2014. Fetal asphyctic preconditioning alters the transcriptional response to perinatal asphyxia. BMC neuroscience, 15, 67.

129. COX, K., STRACKX, E., VLASSAKS, E., SPARNAAIJ, M., ZIMMERMANN, L., VLES, J. \& GAVILANES, D. 2011. Oxidative Stress in Perinatal Asphyxia and Fetal Asphyctic Preconditioning. Pediatric research, 70, 162-162.

130. COX-LIMPENS, K.E.M., STRACKX, E., LA VAN DEN HOVE, D., RA VAN EKKENDONK, J., DE JONG, M., JI ZIMMERMANN, L., WM STEINBUSCH, H., SH VLES, J. \& WD GAVILANES, A. 2015. Fetal asphyctic preconditioning protects against perinatal asphyxiainduced apoptosis and astrogliosis in neonatal brain. CNS \& Neurological Disorders-Drug Targets (Formerly Current Drug Targets-CNS \& Neurological Disorders), 14, 33-40.

131. CROSIERS, D., VERSTRAETEN, A., WAUTERS, E., PICKUT, B., ELINCK, E., CORSMit, E., MATtheiJssens, M., PEETERS, K., DE DEYN, P. \& VAN BROECKHOVEN, C. 2014. Glucerebrosidase mutations: An important genetic risk factor in Flanders-Belgian PD patients associated with a more severe disease phenotype. Movement Disorders, 29, 49.

132. CRUMP, C., SUNDQUIST, K. \& SUNDQUIST, J. 2016. Adult outcomes of preterm birth. Preventive Medicine, 91, 400-401.

133. DA SILVA MEIRELleS, L., FONTES, A. M., COVAS, D. T. \& CAPlAN, A. I. 2009. Mechanisms involved in the therapeutic properties of mesenchymal stem cells. Cytokine \& growth factor reviews, 20, 419-427.

134. DAHL, M., DOYLE, A., OLSSON, K., MÅNSSON, J.-E., MARQUES, A. R., MIRZAIAN, M., AERTS, J. M., EHINGER, M., ROTHE, M. \& MODLICH, U. 2015. Lentiviral Gene Therapy Using Cellular Promoters Cures Type 1 Gaucher Disease in Mice. Molecular Therapy, $23,835-844$

135. DASGUPTA, N., XU, Y.-H., LI, R., PENG, Y., PANDEY, M. K., TINCH, S. L., LIOU, B., INSKEEP, V., ZHANG, W. \& SETCHELL, K. D. 2015. Neuronopathic Gaucher disease: dysregulated mRNAs and miRNAs in brain pathogenesis and effects of pharmacologic chaperone treatment in a mouse model. Human molecular genetics, 24, 7031-7048.

136. DAUER, W. \& PRZEDBORSKI, S. 2003. Parkinson's disease: mechanisms and models. Neuron, 39, 889-909.

137. DAVIDSON, J. O., WASSINK, G., VAN DEN HEUIJ, L. G., BENNET, L. \& GUNN, A. J. 2015. Therapeutic hypothermia for neonatal hypoxic-ischemic encephalopathy-where to from here? Frontiers in neurology, 6.

138. DAVIDSON, J.O., YUILL, C.A., ZHANG, F.G., WASSINK, G., BENNETT, L., GUNN, A.J. (2016). Extending the duration of hypothermia does not further improve white matter protection after ischemia in term-equivalent fetal sheep. Scientific reports, 6 . 
139. DE LA MATA, M., COTÁN, D., OROPESA-ÁVILA, M., GARRIDO-MARAVER, J., CORDERO, M. D., PAZ, M. V., PAVÓN, A. D., ALCOCER-GÓMEZ, E., DE LAVERA, I. \& YBOTGONZÁLEZ, P. 2015. Pharmacological Chaperones and Coenzyme Q10 Treatment Improves Mutant $\beta$-Glucocerebrosidase Activity and Mitochondrial Function in Neuronopathic Forms of Gaucher Disease. Scientific reports, 5, 10903.

140. DE LOUW, A. J., DE Vente, J., STeinbusch, H. P., GAVIlAnes, A. W., STEinBUSCH, H. W., BLANCO, C. E., TROOST, J. \& VLES, J. S. 2002. Apoptosis in the rat spinal cord during postnatal development; the effect of perinatal asphyxia on programmed cell death. Neuroscience, 112, 751-8.

141. DE SMET, H. J., PAQUIER, P., VERHOEVEN, J. \& MARIËN, P. 2013. The cerebellum: its role in language and related cognitive and affective functions. Brain and language, 127, 334-342.

142. De VEnte, J., MARKERINK-VAN itTERSum, M., VAN ABEELEn, J., EMSON, P. C., AXER, H. \& STEINBUSCH, H. W. 2000. NO-mediated cGMP synthesis in cholinergic neurons in the rat forebrain: effects of lesioning dopaminergic or serotonergic pathways on nNOS and cGMP synthesis. Eur J Neurosci, 12, 507-19.

143. DE VENTE, J., STEINBUSCH, H. W. \& SCHIPPER, J. 1987. A new approach to immunocytochemistry of 3',5'-cyclic guanosine monophosphate: preparation, specificity, and initial application of a new antiserum against formaldehyde-fixed 3',5'-cyclic guanosine monophosphate. Neuroscience, 22, 361-73.

144. DELL'ANNA, E., CHEN, Y., ENGIDAWORK, E., ANDERSSON, K., LUBEC, G., LUTHMAN, J. \& HERRERA-MARSCHITZ, M. 1997. Delayed neuronal death following perinatal asphyxia in rat. Exp Brain Res, 115, 105-15.

145. DELl'ANnA, E., CHEN, Y., LOIDL, F., ANDERSSON, K., LUThMAN, J., GOINY, M., RAWAL, R., LINDGREN, T. \& HERRERA-MARSCHITZ, M. 1995. Short-term effects of perinatal asphyxia studied with Fos-immunocytochemistry and in vivo microdialysis in the rat. Experimental neurology, 131, 279-287.

146. DELL'ANNA, M. E., LUTHMAN, J., LINDQVIST, E. \& OLSON, L. 1993. Development of monoamine systems after neonatal anoxia in rats. Brain research bulletin, 32, 159-170.

147. DELL'ANNA, E., CHEN, Y., ENGIDAWORK, E., ANDERSSON, K., LUBEC, G., LUTHMAN, J. \& HERRERA-MARSCHITZ, M. 1997. Delayed neuronal death following perinatal asphyxia in rat. Experimental brain research, 115, 105-115.

148. DELONG, M. R. \& WICHMANN, T. 2007. Circuits and circuit disorders of the basal ganglia. Archives of neurology, 64, 20-24.

149. DeRMENTZAKI, G., Dimitriou, E., XilOuRI, M., MiCHELAKAKIS, H. \& STEFANIS, L. 2013. Loss of $\beta$-glucocerebrosidase activity does not affect alpha-synuclein levels or lysosomal function in neuronal cells. PloS one, 8, e60674.

150. Devoto, V. P., BOGETTI, M. \& DE PlaZAS, S. F. 2013. Developmental and hypoxiainduced cell death share common ultrastructural and biochemical apoptotic features in the central nervous system. Neuroscience, 252, 190-200.

151. DIEDERICH, N. J. \& MCINTYRE, D. J. 2012. Sleep disorders in Parkinson's disease: many causes, few therapeutic options. Journal of the neurological sciences, 314, 12-19.

152. DONEGA, V., NIJBOER, C. H., VAN VELTHOVEN, C. T., YOUSSEF, S. A., DE BRUIN, A., VAN BEL, F., KAVELAARS, A. \& HEIJNEN, C. J. 2015. Assessment of long-term safety and efficacy of intranasal mesenchymal stem cell treatment for neonatal brain injury in the mouse. Pediatric research, 78, 520. 
153. DONEGA, V., VAN VELThOVEn, C. T., NIJBOER, C. H., VAN BEL, F., KAS, M. J., KAVELAARS, A. \& HEIJNEN, C. J. 2013. Intranasal mesenchymal stem cell treatment for neonatal brain damage: long-term cognitive and sensorimotor improvement. PLoS One, 8, e51253.

154. DONG, C., WEI, P., JIAN, X., GIBBS, R., BOERWINKLE, E., WANG, K. \& LIU, X. 2015. Comparison and integration of deleteriousness prediction methods for nonsynonymous SNVs in whole exome sequencing studies. Human molecular genetics, 24, 2125-2137.

155. DORFMAN, V. B., REY-FUnes, M., BAYONA, J. C., LÓPEZ, E. M., COIRINI, H. \& LOIDL, C. F. 2009. Nitric oxide system alteration at spinal cord as a result of perinatal asphyxia is involved in behavioral disabilities: hypothermia as preventive treatment. Journal of neuroscience research, 87, 1260-1269.

156. DORSEY, E., CONSTANTINESCU, R., THOMPSON, J., BIGLAN, K., HOLLOWAY, R. KIEBURTZ, K., MARSHALL, F., RAVINA, B., SCHIFITTO, G. \& SIDEROWF, A. 2007 Projected number of people with Parkinson disease in the most populous nations, 2005 through 2030. Neurology, 68, 384-386.

157. DRACHMAN, D. A. 2014. The amyloid hypothesis, time to move on: Amyloid is the downstream result, not cause, of Alzheimer's disease. Alzheimer's \& Dementia, 10, 372-380.

158. DROMMELSCHMIDT, K., SERDAR, M., BENDIX, I., HERZ, J., BERTLING, F., PRAGER, S., KELlER, M., LUDWIG, A.-K., DUHAN, V. \& RADTKE, S. 2017. Mesenchymal stem cell-derived extracellular vesicles ameliorate inflammation-induced preterm brain injury. Brain, behavior, and immunity, 60, 220-232.

159. DRURY, P. P., DAVIDSON, J. O., MATHAI, S., VAN DEN HEUIJ, L. G., JI, H., BENNET, L., TAN, S., SILVERMAN, R. B. \& GUNN, A. J. 2014. nNOS inhibition during profound asphyxia reduces seizure burden and improves survival of striatal phenotypic neurons in preterm fetal sheep. Neuropharmacology, 83, 62-70.

160. DURAN, R., MENCACCI, N. E., ANGEli, A. V., SHOAI, M., DEAS, E., HOULDEN, H., MEHTA, A., HUGHES, D., COX, T. M. \& DEEGAN, P. 2013. The glucocerobrosidase E326K variant predisposes to Parkinson's disease, but does not cause Gaucher's disease. Movement Disorders, 28, 232-236.

161. EDWARDS, A. D., BROCKLEHURST, P., GUNN, A. J., HALLIDAY, H., JUSZCZAK, E., LEVENE, M., STROHM, B., THORESEN, M., WHITELAW, A. \& AZZOPARDI, D. 2010. Neurological outcomes at 18 months of age after moderate hypothermia for perinatal hypoxic ischaemic encephalopathy: synthesis and meta-analysis of trial data. BMJ, 340, c363.

162. EHRLICH, M. E. 2012. Huntington's disease and the striatal medium spiny neuron: cellautonomous and non-cell-autonomous mechanisms of disease. Neurotherapeutics, 9, 270-284.

163. EL-KHODOR, B. \& BOKSA, P. 1998. Birth insult increases amphetamine-induced behavioral responses in the adult rat. Neuroscience, $87,893-904$.

164. EL-KHODOR, B. \& BOKSA, P. 2001. Caesarean section birth produces long term changes in dopamine D1 receptors and in stress-induced regulation of D3 and D4 receptors in the rat brain. Neuropsychopharmacology, 25, 423-439.

165. EL-KHODOR, B. F. \& BOKSA, P. 1997. Long-term reciprocal changes in dopamine levels in prefrontal cortex versus nucleus accumbens in rats born by Caesarean section compared to vaginal birth. Experimental neurology, 145, 118-129.

166. EL-KHODOR, B. F. \& BOKSA, P. 2000. Transient birth hypoxia increases behavioral responses to repeated stress in the adult rat. Behavioural brain research, 107, 171-175. 
167. EL-KHODOR, B. F. \& BOKSA, P. 2003. Differential vulnerability of male versus female rats to long-term effects of birth insult on brain catecholamine levels. Experimental neurology, $182,208-219$.

168. EL-KHODOR, B. F., FLORES, G., SRIVASTAVA, L. K. \& BOKSA, P. 2004. Effects of birth insult and stress at adulthood on excitatory amino acid receptors in adult rat brain. Synapse, 54, 138-146.

169. ENGIDAWORK, E., CHEN, Y., DELL'ANNA, E., GOINY, M., LUBEC, G., UNGERSTEDT, U., ANDERSSON, K. \& HERRERA-MARSCHITZ, M. 1997. Effect of perinatal asphyxia on systemic and intracerebral $\mathrm{pH}$ and glycolysis metabolism in the rat. Experimental neurology, 145, 390-396.

170. ENGIDAWORK, E., LOIDL, F., CHEN, Y., KOHLHAUSER, C., STOECKLER, S., DELL'ANNA, E., LUBEC, B., LUBEC, G., GOINY, M. \& GROSS, J. 2001. Comparison between hypothermia and glutamate antagonism treatments on the immediate outcome of perinatal asphyxia. Experimental brain research, 138, 375-383.

171. ERGEnEKON, E., GÜCÜYENER, K., ERBAŞ, D., ARAL, S., KOÇ, E. \& ATAlAY, Y. 2004. Cerebrospinal fluid and serum vascular endothelial growth factor and nitric oxide levels in newborns with hypoxic ischemic encephalopathy. Brain and Development, 26, 283-286.

172. ESKELINEN, E.-L. 2006. Roles of LAMP-1 and LAMP-2 in lysosome biogenesis and autophagy. Molecular aspects of medicine, 27, 495-502.

173. EUNSON, P. 2015. The long-term health, social, and financial burden of hypoxic-ischaemic encephalopathy. Developmental Medicine \& Child Neurology, 57, 48-50.

174. FAN, M. M. \& RAYMOND, L. A. 2007. N-methyl-D-aspartate (NMDA) receptor function and excitotoxicity in Huntington's disease. Progress in neurobiology, 81, 272-293.

175. FARRER, M. J., WILliAMS, L. N., ALGOM, A. A., KACHERGUS, J., HULiHAN, M. M., ROSS, O. A., RAJPUT, A., PAPAPETROPOUlOS, S., MASH, D. C. \& DICKSON, D. W. 2009. Glucosidase-beta variations and Lewy body disorders. Parkinsonism \& related disorders, 15, 414-416.

176. FATHALLAH, A. M., BANKERT, R. B. \& BALU-IYER, S. V. 2013. Immunogenicity of subcutaneously administered therapeutic proteins - a mechanistic perspective. The AAPS journal, 15, 897-900.

177. FERNÁNDEZ-NOGALES, M., CABRERA, J. R., SANTOS-GALINDO, M., HOOZEMANS, J. J., FERRER, I., ROZEMUlleR, A. J., HERNÁNDEZ, F., AVILA, J. \& LUCAS, J. J. 2014. Huntington's disease is a four-repeat tauopathy with tau nuclear rods. Nature medicine, $20,881-885$.

178. FERRIERO, D., ARCAVI, L. \& SIMON, R. 1990. Ontogeny of excitotoxic injury to nicotinamide adenine dinucleotide phosphate diaphorase reactive neurons in the neonatal rat striatum. Neuroscience, 36, 417-424.

179. FISCHER, U. M., HARTING, M. T., JIMENEZ, F., MONZON-POSADAS, W. O., XUE, H., SAVITZ, S. I., LAINE, G. A. \& COX JR, C. S. 2009. Pulmonary passage is a major obstacle for intravenous stem cell delivery: the pulmonary first-pass effect. Stem cells and development, 18, 683-692.

180. FISHBEIN, I., KUO, Y.-M., GIASSON, B. I. \& NUSSBAUM, R. L. 2014. Augmentation of phenotype in a transgenic Parkinson mouse heterozygous for a Gaucher mutation. Brain, 137, pp.3235-3247. 
181. Fleiss, B., COLEMAN, H., CASTILlO-MELENDEZ, M., IRELAND, Z., WALKER, D. \& PARKINGTON, H. 2011. Effects of birth asphyxia on neonatal hippocampal structure and function in the spiny mouse. International Journal of Developmental Neuroscience, 29, 757-766.

182. FleisS, B., PARKIngton, H. C., COLEMAN, H. A., DICKINSON, H., YAWNO, T., CASTILLO-MELENDEZ, M., HIRST, J. J. \& WALKER, D. W. 2012. Effect of maternal administration of allopregnanolone before birth asphyxia on neonatal hippocampal function in the spiny mouse. Brain research, 1433, 9-19.

183. FLORES-BALTER, G., CORDOVA-JADUE, H., CHITI-MORALES, A., LESPAY, C., ESPINAMARCHANT, P., FALCON, R., GRINSPUN, N., SANCHEZ, J., BUSTAMANTE, D. \& MORALES, P. 2016. Effect of perinatal asphyxia on tuberomammillary nucleus neuronal density and object recognition memory: A possible role for histamine? Behavioural Brain Research, 313, 226-232.

184. FLORES, C., STEWART, J., SALMASO, N., ZHANG, Y. \& BOKSA, P. 2002. Astrocytic basic fibroblast growth factor expression in dopaminergic regions after perinatal anoxia. Biological psychiatry, 52, 362-370.

185. FREEMAN, T. B., SANBERG, P. R. \& ISACSON, O. 1995. Development of the human striatum: implications for fetal striatal transplantation in the treatment of Huntington's disease. Cell transplantation, 4, 539-545.

186. FRIEDMAN, D. S., REPKA, M. X., KATZ, J., GIORDANO, L., IBIRONKE, J., HAWSE, P. \& TIELSCH, J. M. 2009. Prevalence of amblyopia and strabismus in white and African American children aged 6 through 71 months: the Baltimore Pediatric Eye Disease Study. Ophthalmology, 116, 2128-2134. e2.

187. FRIZZO, J. K., CARDOSO, M. P., DE ASSIS, A. M., PERRY, M. L., VOLONTÉ, C. \& FRIZZO, M. E. 2010. Effects of acute perinatal asphyxia in the rat hippocampus. Cellular and molecular neurobiology, 30, 683-692.

188. Frousios, K., Iliopoulos, C. S., SChlitT, T. \& SIMPSON, M. A. 2013. Predicting the functional consequences of non-synonymous DNA sequence variants - evaluation of bioinformatics tools and development of a consensus strategy. Genomics, 102, 223-228.

189. GAlEANO, P., BlANCO, E., TORNATORE, T. M. L., ROMERO, J. I., HOLUBIEC, M. I., DE FONSECA, F. R. \& CAPANI, F. 2014. Life-long environmental enrichment counteracts spatial learning, reference and working memory deficits in middle-aged rats subjected to perinatal asphyxia. Frontiers in behavioral neuroscience, 8.

190. GaleAno, P., ROMERO, J. I., LUQUe-rojAs, M. J., SUÁREZ, J., HOLUBieC, M. I., BISAGNO, V., SANTÍN, L. J., FONSECA, F. R., CAPANI, F. \& BLANCO, E. 2013. Moderate and severe perinatal asphyxia induces differential effects on cocaine sensitization in adult rats. Synapse, 67, 553-567.

191. GAN-OR, Z., AMSHALOM, I., BAR-SHIRA, A., GANA-WEISZ, M., MIRELMAN, A., MARDER, K., BRESSMAN, S., GILADI, N. \& ORR-URTREGER, A. 2015a. The Alzheimer disease BIN1 locus as a modifier of GBA-associated Parkinson disease. Journal of neurology, $1-5$.

192. GAN-OR, Z., AMSHALOM, I., KILARSKI, L. L., BAR-SHIRA, A., GANA-WEISZ, M., MIRELMAN, A., MARDER, K., BRESSMAN, S., GILADI, N. \& ORR-URTREGER, A 2015b. Differential effects of severe vs mild GBA mutations on Parkinson disease. Neurology, $84,880-887$.

193. GAN-OR, Z., GILADI, N., ROZOVSKI, U., SHIFRIN, C., ROSNER, S., GUREVICH, T., BAR-SHIRA, A. \& ORR-URTREGER, A. 2008. Genotype-phenotype correlations between GBA mutations and Parkinson disease risk and onset. Neurology, 70, 2277-2283. 
194. GARCIA-REITBÖCK, P., ANICHTCHIK, O., BELLUCCI, A., IOVINO, M., BALLINI, C., FINEBERG, E., GHETTI, B., DELla CORTE, L., SPANO, P. \& TOFARIS, G. K. 2010. SNARE protein redistribution and synaptic failure in a transgenic mouse model of Parkinson's disease. Brain, 133, 2032-2044.

195. GATTO, C. L. \& BROADIE, K. 2010. Genetic controls balancing excitatory and inhibitory synaptogenesis in neurodevelopmental disorder models. Frontiers in synaptic neuroscience, 2

196. GEGG, M. E., BURKE, D., HEALES, S. J., COOPER, J. M., HARDY, J., WOOD, N. W. \& SCHAPIRA, A. H. 2012. Glucocerebrosidase deficiency in substantia nigra of parkinson disease brains. Annals of neurology, 72, 455-463.

197. GEGG, M. E., SWEET, L., WANG, B. H., SHIHABUDDIN, L. S., SARDI, S. P. \& SCHAPIRA, A. H. 2015. No evidence for substrate accumulation in Parkinson brains with GBA mutations. Movement Disorders, 30, 1085-1089.

198. GELDENHUYS, G., GLANZMANN, B., LOMBARD, D., BOOlAY, S., CARR, J. \& BARDIEN, S. 2014. Identification of a common founder couple for 40 South African Afrikaner families with Parkinson's disease. SAMJ: South African Medical Journal, 104, 413-419.

199. GIBB, W. \& LEES, A. 1988. A comparison of clinical and pathological features of young-and old-onset Parkinson's disease. Neurology, 38, 1402-1402.

200. GINNS, E. I., MAK, S. K.-K., KO, N., KARLGREN, J., AKBARIAN, S., CHOU, V. P., GUO, Y., LIM, A., SAMUELSSON, S. \& LAMARCA, M. L. 2014. Neuroinflammation and $\alpha$-synuclein accumulation in response to glucocerebrosidase deficiency are accompanied by synaptic dysfunction. Molecular genetics and metabolism, 111, 152-162.

201. GLICKMAN, A., BODAMER, O., JOHNSON, B., NICHOLS, W., PULLMAN, M., ORTEGA, R., JOHANNES, B., RAYMOND, D., OZELIUS, L. \& BIELAWSKI, J. 2015. Peripheral $\beta$-glucocerebrosidase enzyme activity and lipidomics in ß-glucocerebrosidase (GBA1) mutation Parkinson disease (P2. 149). Neurology, 84, P2. 149.

202. GOEDERT, M., JAKES, R., CROWTHER, R., SIX, J., LÜBKE, U., VANDERMEEREN, M., CRAS, P., TROJANOWSKI, J. \& LEE, V. 1993. The abnormal phosphorylation of tau protein at Ser-202 in Alzheimer disease recapitulates phosphorylation during development. Proceedings of the National Academy of Sciences, 90, 5066-5070.

203. GOKER-ALPAN, O., LOPEZ, G., VITHAYATHIL, J., DAVIS, J., HALLETT, M. \& SIDRANSKY, E. 2008. The spectrum of parkinsonian manifestations associated with glucocerebrosidase mutations. Archives of neurology, 65, 1353-1357.

204. GOKER-ALPAN, O., MASDEU, J. C., KOHN, P. D., IANNI, A., LOPEZ, G., GRODEN, C., CHAPMAN, M. C., CROPP, B., EISENBERG, D. P. \& MANIWANG, E. D. 2012. The neurobiology of glucocerebrosidase-associated parkinsonism: a positron emission tomography study of dopamine synthesis and regional cerebral blood flow. Brain, 135, 2440-2448.

205. GOKER-ALPAN, O., SCHIFFMANN, R., LAMARCA, M., NUSSBAUM, R., MCINERNEYLEO, A. \& SIDRANSKY, E. 2004. Parkinsonism among Gaucher disease carriers. Journal of medical genetics, 41, 937-940.

206. GOLDMAN, S. M. 2014. Environmental toxins and Parkinson's disease. Annual review of pharmacology and toxicology, 54, 141-164.

207. GONÇALVES, V. M., D'ALMEIDA, V., MÜLlER, K. B., REAL, F. \& MORTARA, R. A. 2014. Lysosomal integral membrane protein 2 (LIMP-2) restricts the invasion of Trypanosoma cruzi extracellular amastigotes through the activity of the lysosomal enzyme $\beta$ glucocerebrosidase. Microbes and Infection, 16, 253-260. 
208. GORGES, M., MÜLlER, H.-P., LULÉ, D., PINKHARDT, E. H., LUDOLPH, A. C., KASSUBEK, J. \& CONSORTIUM, L. 2015. The association between alterations of eye movement control and cerebral intrinsic functional connectivity in Parkinson's disease. Brain imaging and behavior, 1-13.

209. GRAhAm, E. M., RUIS, K. A., HARTMAN, A. L., NORThingtON, F. J. \& FOX, H. E. 2008. A systematic review of the role of intrapartum hypoxia-ischemia in the causation of neonatal encephalopathy. American journal of obstetrics and gynecology, 199, 587-595.

210. GREEFF, J. M. 2007. Deconstructing Jaco: genetic heritage of an Afrikaner. Annals of human genetics, 71, 674-688.

211. GRIGORENKO, E. L. 2005. A conservative meta-analysis of linkage and linkage-association studies of developmental dyslexia. Scientific Studies of Reading, 9, 285-316.

212. GRIMALDI, M., ROMER, I., DE APODACA, M. G., ITURBE, L., CATANIA, I., GONZÁLEZ, J., KOLLIKER-FRES, R., BARRETO, G. \& CAPANI, F. 2012. Early changes in the synapses of the neostriatum induced by perinatal asphyxia. Nutritional neuroscience, 15 , 103-110.

213. GRIMM, M. O., HUNDSDÖRFER, B., GROESGEN, S., METT, J., ZIMMER, V. C., STAHLMANN, C. P., HAUPENTHAL, V. J., ROTHHAAR, T. L., LEHMANN, J. \& PAETZOLD, A. 2014. PS dependent APP cleavage regulates glucosylceramide synthase and is affected in Alzheimer's disease. Cellular Physiology and Biochemistry, 34, 92-110.

214. GROENENDAAL, F., LAMMERS, H., SMIT, D. \& NIKKELS, P. G. 2006. Nitrotyrosine in brain tissue of neonates after perinatal asphyxia. Archives of Disease in Childhood-Fetal and Neonatal Edition, 91, F429-F433.

215. GROSS, J., ANDERSSON, K., CHEN, Y., MÜLLER, I., ANDREEVA, N. \& HERRERAMARSCHITZ, M. 2005. Effect of perinatal asphyxia on tyrosine hydroxylase and D2 and D1 dopamine receptor mRNA levels expressed during early postnatal development in rat brain. Molecular brain research, 134, 275-281.

216. GROSS, J., MÜLLER, I., CHEN, Y., ELIZALDE, M., LECLERE, N., HERRERA-MARSCHITZ M. \& ANDERSSON, K. 2000. Perinatal asphyxia induces region-specific long-term changes in mRNA levels of tyrosine hydroxylase and dopamine D 1 and D 2 receptors in rat brain. Molecular brain research, 79, 110-117.

217. GRUSCHUS, J. M. 2015. Did $\alpha$-Synuclein and Glucocerebrosidase Coevolve? Implications for Parkinson's Disease. PloS one, 10, e0133863.

218. GRUSCHUS, J. M., JIANG, Z., YAP, T. L., HILL, S. A., GRISHAEV, A., PISZCZEK, G., SIDRANSKY, E. \& LEE, J. C. 2015. Dissociation of glucocerebrosidase dimer in solution by its co-factor, saposin C. Biochemical and Biophysical Research Communications, 457, 561-566.

219. GUILlET, R., EDWARDS, A. D., THORESEN, M., FERRIERO, D. M., GLUCKMAN, P. D., WHITELAW, A., GUNN, A. J. \& COOLCAP TRIAL, G. 2012. Seven- to eight-year follow-up of the CoolCap trial of head cooling for neonatal encephalopathy. Pediatric research, 71, 205-9.

220. GUNES, T., OZTURK, M. A., KOKLU, E., KOSE, K. \& GUNES, I. 2007. Effect of allopurinol supplementation on nitric oxide levels in asphyxiated newborns. Pediatric neurology, $36,17-24$.

221. GUNN, A. J. \& BENNET, L. 2008. Brain cooling for preterm infants. Clinics in perinatology, $35,735-748$. 
222. GUNninG, P. W., SCHEVZOV, G., KEE, A. J. \& HARDEMAN, E. C. 2005. Tropomyosin isoforms: divining rods for actin cytoskeleton function. Trends in cell biology, 15, 333-341.

223. GUSELLA, J. F. \& MACDONALD, M. E. 2009. Huntington's disease: the case for genetic modifiers. Genome medicine, 1,80

224. HAGBERG, H., GRESSENS, P. \& MALLARD, C. 2012. Inflammation during fetal and neonatal life: implications for neurologic and neuropsychiatric disease in children and adults. Annals of neurology, 71, 444-457.

225. HAN, F., GRIMES, D. A., LI, F., WANG, T., YU, Z., SONG, N., WU, S., RACACHO, L. \& BULMAN, D. E. 2015. Mutations in the Glucocerebrosidase Gene Are Common in Patients with Parkinson's Disease from Eastern Canada. International Journal of Neuroscience, 1-19.

226. HANSON, L. R., FINE, J. M., SVITAK, A. L. \& FALTESEK, K. A. 2013. Intranasal administration of CNS therapeutics to awake mice. JoVE (Journal of Visualized Experiments), e4440-e4440.

227. HAYLETT, W. L., KEYSER, R. J., DU PLESSIS, M. C., VAN DER MERWE, C., BLANCK ENBERG, J., LOMBARD, D., CARR, J. \& BARDIEN, S. 2012. Mutations in the parkin gene are a minor cause of Parkinson's disease in the South African population. Parkinsonism \& related disorders, $18,89-92$

228. HEBELS, D. G., JENnEN, D. G., KLEINJANS, J. C. \& DE KOK, T. M. 2009. Molecular signatures of N-nitroso compounds in Caco-2 cells: implications for colon carcinogenesis Toxicological sciences, 108, 290-300.

229. HEDTJÄRN, M., LEVERIN, A.-L., ERIKSSON, K., BLOMGREN, K., MALLARD, C. \& HAGBERG, H. 2002. Interleukin-18 involvement in hypoxic-ischemic brain injury. Journal of Neuroscience, 22, 5910-5919.

230. HENEKA, M. T., KUMMER, M. P. \& LATZ, E. 2014. Innate immune activation in neurodegenerative disease. Nature reviews. Immunology, 14, 463

231. HERBison, A. E., SIMONIAN, S. X., NORRIS, P. J. \& EMSON, P. C. 1996. Relationship of neuronal nitric oxide synthase immunoreactivity to GnRH neurons in the ovariectomized and intact female rat. J Neuroendocrinol, 8, 73-82.

232. HERRERA-MARSCHITZ, M., LOIDL, C., ANDERSSON, K. \& UNGERSTEDT, U. 1993 Prevention of mortality induced by perinatal asphyxia: Hypothermia or glutamate antagonism? Amino Acids, 5, 413-419.

233. HERRERA-MARSCHITZ, M., MORALES, P., LEYTON, L., BUSTAMANTE, D., KLAWITTER, V., ESPINA-MARCHANT, P., ALLENDE, C., LISBOA, F., CUNICH, G. \& JARACAVIERES, A. 2011. Perinatal asphyxia: current status and approaches towards neuroprotective strategies, with focus on sentinel proteins. Neurotoxicity research, 19, 603-627.

234. HERRERA-MARSCHITZ, M., NEIRA-PEÑA, T., LEYTON, L., GEBICKE-HAERTER, P., ROJAS-MANCILlA, E., MORALES, P. \& BUSTAMANTE, D. 2015. Short-and longterm consequences of perinatal asphyxia: looking for neuroprotective strategies. Perinatal Programming of Neurodevelopment. Springer.

235. HERRERA, M. I., OTERO-LOSADA, M., UDOVIN, L. D., KUSNIER, C., KÖLLIKERFRERS, R., DE SOUZA, W. \& CAPANI, F. 2017. Could Perinatal Asphyxia Induce a Synaptopathy? New Highlights from an Experimental Model. Neural plasticity, 2017.

236. HERRERA, M. I., ROMERO, J., DE FONSECA, F. R., BLANCO, E. \& CAPANI, F. 2014. Behavioral Effect of Oleoylethanolamide on Perinatal Asphyxia. Journal of Advanced Neuroscience Research, 1, 22-26. 
237. HESS, D. C., WECHSLER, L. R., CLARK, W. M., SAVITZ, S. I., FORD, G. A., CHIU, D., YAVAGAL, D. R., UCHINO, K., LIEBESKIND, D. S. \& AUCHUS, A. P. 2017. Safety and efficacy of multipotent adult progenitor cells in acute ischaemic stroke (MASTERS): a randomised, double-blind, placebo-controlled, phase 2 trial. The Lancet Neurology, 16, 360-368.

238. HILL, A. B. 1965. The environment and disease: association or causation? Proceedings of the Royal Society of Medicine, 58, 295-300.

239. HIROKAWA, N., NODA, Y., TANAKA, Y. \& NIWA, S. 2009. Kinesin superfamily motor proteins and intracellular transport. Nature reviews. Molecular cell biology, 10, 682.

240. HIRSCH, C., CAMPANO, L. M., WÖHRLE, S. \& HECHT, A. 2007. Canonical Wnt signaling transiently stimulates proliferation and enhances neurogenesis in neonatal neural progenitor cultures. Experimental cell research, 313, 572-587.

241. HOEGER, H., ENGELMANN, M., BERNERT, G., SEIDL, R., BUBNA-LITTITZ, H., MOSGOELLER, W., LUBEC, B. \& LUBEC, G. 2000. Long term neurological and behavioral effects of graded perinatal asphyxia in the rat. Life Sci, 66, 947-62.

242. holmay, M. J., TerpstRA, M., COles, L. D., MiShrA, U., AHLSKOG, M., ÖZ, G., CLOYD, J. C. \& TUITE, P. J. 2013. N-acetylcysteine boosts brain and blood glutathione in gaucher and Parkinson diseases. Clinical neuropharmacology, 36, 103-106.

243. HOPFNeR, F., SChulte, E. C., MOllenhauer, B., BEREZnAi, B., KNAUF, F., LICHTNER, P., ZIMPRICH, A., HAUBENBERGER, D., PIRKER, W. \& BRÜCKE, T. 2013. The role of SCARB2 as susceptibility factor in Parkinson's disease. Movement Disorders, $28,538-540$.

244. HOROWITZ, M., PASMANIK-CHOR, M., RON, I. \& KOLODNY, E. H. 2011. The enigma of the E326K mutation in acid $\beta$-glucocerebrosidase. Molecular genetics and metabolism, $104,35-38$.

245. HOYT, C. S. \& TAYLOR, D. 2012. Pediatric ophthalmology and strabismus, Elsevier Health Sciences.

246. HRUSKA, K. S., LAMARCA, M. E., SCOTT, C. R. \& SIDRANSKY, E. 2008. Gaucher disease: mutation and polymorphism spectrum in the glucocerebrosidase gene (GBA). Human mutation, 29, 567-583.

247. IKENO, S., NAGATA, N., YOSHIDA, S., TAKAHASHI, H., KIGAWA, J. \& TERAKAWA N. 2000. Immature Brain Injury via Peroxynitrite Production Induced by Inducible Nitric Oxide Synthase after Hypoxia-Ischemia in Rats. Journal of Obstetrics and Gynaecology Research, 26, 227-234.

248. INOUE, H., MATSUSHIGE, T., HASEGAWA, S., ABE, A., IIDA, Y., INOUE, T. \& ICHIYAMA, T. 2012. Elevation of tau protein levels in the cerebrospinal fluid of children with West syndrome. Epilepsy research, 102, 8-12.

249. INZELBERG, R., RAMIREZ, J. A., NISIPEANU, P. \& OPHIR, A. 2004. Retinal nerve fiber layer thinning in Parkinson disease. Vision research, 44, 2793-2797.

250. IRANZO, A., TOlOSA, E., GELPI, E., MOLINUEVO, J. L., VALlDEORIOLA, F., SERRADELL, M., SANCHEZ-VALLE, R., VILASECA, I., LOMENA, F. \& VILAS, D. 2013. Neurodegenerative disease status and post-mortem pathology in idiopathic rapid-eye-movement sleep behaviour disorder: an observational cohort study. The Lancet Neurology, 12, 443-453.

251. IRELAND, Z., DICKINSON, H., SNOW, R. \& WALKER, D. W. 2008. Maternal creatine: does it reach the fetus and improve survival after an acute hypoxic episode in the spiny mouse (Acomys cahirinus)? American journal of obstetrics and gynecology, 198, 431. e1-431. e6. 
252. IRWIN, D. J., LEE, V. M.-Y. \& TROJANOWSKI, J. Q. 2013. Parkinson's disease dementia: convergence of [alpha]-synuclein, tau and amyloid-[beta] pathologies. Nature Reviews Neuroscience, 14, 626-636.

253. JACK, A., AMATO, D., MORRIS, G. \& CHOY, F. Y. 2014. Two novel mutations in glucocerebrosidase, C23W and IVS7- $1 \mathrm{G}>\mathrm{A}$, identified in Type 1 Gaucher patients heterozygous for N370S. Gene, 538, 84-87.

254. JACOBS, S. A., PINXTEREN, J., ROOBROUCK, V. D., LUYCKX, A., HOF, W. V. T. DEANS, R., VERFAILlie, C. M., WAER, M., BILliAU, A. D. \& VAN GOOL, S. W. 2013a. Human multipotent adult progenitor cells are nonimmunogenic and exert potent immunomodulatory effects on alloreactive T-cell responses. Cell transplantation, 22, 19151928

255. JACOBS, S. A., ROOBROUCK, V. D., VERFAillie, C. M. \& VAN GOOL, S. W. 2013b. Immunological characteristics of human mesenchymal stem cells and multipotent adult progenitor cells. Immunology and cell biology, 91, 32-39.

256. JACOBS, S. E., BERG, M., HUNT, R., TARNOW-MORDI, W. O., INDER, T. E. \& DAVIS, P. G. 2013c. Cooling for newborns with hypoxic ischaemic encephalopathy. Cochrane Database Syst Rev, 1, CD003311.

257. JAMrozIK, Z., LUGOWSKA, A., KOZIOROWSKI, D., FRIEDMAN, A., SLAWEK, J., JANIK, P., POTULSKA-CHROMIK, A., KUZMA-KOZAKIEWICZ, M., WISNIEWSKA, A. \& SZUBIGA, M. 2014. Mutations L444P and N370S in the GBA gene in Polish patients with early and later onset Parkinson's disease. Movement Disorders, 29,53.

258. JAMROZIK, Z., LUGOWSKA, A., SLAWEK, J. \& KWIECINSKI, H. 2010. Glucocerebrosidase mutations p. L444P and p. N370S are not associated with multisystem atrophy, progressive supranuclear palsy and corticobasal degeneration in Polish patients. Journal of neurology, 257, 459-460.

259. JANDER, S., SCHROETER, M., STOLL, G. 2000. Role of NMDA receptor signaling in the regulation of inflammatory gene expression after focal brain ischemia.Journal of neuroimmunology, 109, 181-187.

260. JANKOVIC, M., DOBRICIC, V., KRESOJEVIC, N., ZARKOVIC, M., SVETEL, M., NOVAKOVIC, I. \& KOSTIC, V. 2014. Glucocerebrosidase-associated Parkinson's disease in Serbian population: From epidemiological studies to clinical implementation. Movement Disorders, 29,53.

261. JELLEMA, R. K., LIMA PASSOS, V., ZWANENBURG, A., OPHELDERS, D., DE MUNTER, S., VANDERLOCHT, J., GERMERAAD, W., KUYPERS, E., COLLINS, J. \& CLEUTJENS, J. 2013a. Cerebral inflammation and mobilization of the peripheral immune system following global hypoxia-ischemia in preterm sheep. J Neuroinflammation, 10, 2094-10.

262. JELLEMA, R. K., OPHELDERS, D. R., ZWANENBURG, A., NIKIFOROU, M., DELHAAS, T., ANDRIESSEN, P., MAYS, R. W., DEANS, R., GERMERAAD, W. T. \& WOLFS, T. G. 2015. Multipotent adult progenitor cells for hypoxic-ischemic injury in the preterm brain. Journal of neuroinflammation, 12, 241

263. JELLEMA, R. K., WOLFS, T. G., PASSOS, V. L., ZWANENBURG, A., OPHELDERS, D. R., KUYPERS, E., HOPMAN, A. H., DUDINK, J., STEINBUSCH, H. W. \& ANDRIESSEN, P. 2013b. Mesenchymal stem cells induce T-cell tolerance and protect the preterm brain after global hypoxia-ischemia. PloS one, 8, e73031.

264. JI, H., TAN, S., IGARASHI, J., LI, H., DERRICK, M., MARTÁSEK, P., ROMAN, L. J., VÁSQUEZ-VIVAR, J., POULOS, T. L. \& SILVERMAN, R. B. 2009. Selective neuronal nitric oxide synthase inhibitors and the prevention of cerebral palsy. Annals of neurology, 65, 209-217. 
265. JOHNSON, S., FAWKE, J., HENNESSY, E., ROWELL, V., THOMAS, S., WOLKE, D. \& MARLOW, N. 2009a. Neurodevelopmental disability through 11 years of age in children born before 26 weeks of gestation. Pediatrics, 124, e249-e257.

266. JOHNSON, S., HENNESSY, E., SMITH, R., TRIKIC, R., WOLKE, D. \& MARLOW, N. 2009b. Academic attainment and special educational needs in extremely preterm children at 11 years of age: the EPICure study. Archives of Disease in Childhood-Fetal and Neonatal Edition, 94, F283-F289.

267. JOHnSOn, S., HOllis, C., KOCHhAR, P., HENNESSY, E., WOlKE, D. \& MARLOW, N. 2010. Psychiatric disorders in extremely preterm children: longitudinal finding at age 11 years in the EPICure study. Journal of the American Academy of Child \& Adolescent Psychiatry, 49, 453-463. e1.

268. JOHNSON, S. \& MARLOW, N. 2017. Early and long-term outcome of infants born extremely preterm. Archives of disease in childhood, 102, 97-102

269. JOHNSTON, M. V. 2005. Excitotoxicity in perinatal brain injury. Brain pathology, 15, 234-240.

270. JUÁREZ, I., GRATTON, A. \& FLORES, G. 2008. Ontogeny of altered dendritic morphology in the rat prefrontal cortex, hippocampus, and nucleus accumbens following Cesarean delivery and birth anoxia. Journal of Comparative Neurology, 507, 1734-1747.

271. KAMINSKA, B. 2005. MAPK signalling pathways as molecular targets for anti-inflammatory therapy - from molecular mechanisms to therapeutic benefits. Biochimica et Biophysica Acta (BBA)-Proteins and Proteomics, 1754, 253-262.

272. KANG, H. \& SHIN, J.-H. 2015. Repression of rRNA transcription by PARIS contributes to Parkinson's disease. Neurobiology of disease, 73, 220-228.

273. KASTNER, P., MOSGOELLER, W., FANG-KIRCHER, S., KITZMUELLER, E., KIRCHNER, L., HOEGER, H., SEITHER, P., LUBEC, G. \& LUBEC, B. 2003. Deficient brain RNA polymerase and altered nucleolar structure persists until day 8 after perinatal asphyxia of the rat. Pediatric research, 53, 62-71.

274. KEATINGE, M., BUI, H., MENKE, A., CHEN, Y.-C., SOKOL, A. M., BAI, Q., ELleTt, F., DA COSTA, M., BURKE, D. \& GEGG, M. 2015a. Glucocerebrosidase 1 deficient Danio rerio mirror key pathological aspects of human Gaucher disease and provide evidence of early microglial activation preceding alpha-synuclein-independent neuronal cell death. $\mathrm{Hu}-$ man molecular genetics, 24, 6640-6652.

275. KEATINGE, M., DACOSTA, M., MENKE, A., CHEN, Y.-C., BUI, H. H., O'NEILL, M. \& BANDMANN, O. 2015b. ZEBRAFISH AS A MODEL OF GLUCOCEREBROSIDASE 1 (GBA1) DEFICIENCY. Journal of Neurology, Neurosurgery \& Psychiatry, 86, e4-e4.

276. KELDER, T., VAN IERSEL, M. P., HANSPERS, K., KUTMON, M., CONKLIN, B. R., EVELO, C. T. \& PICO, A. R. 2011. WikiPathways: building research communities on biological pathways. Nucleic acids research, 40, D1301-D1307.

277. KEYNES, R. G. \& GARTHWAITE, J. 2004. Nitric oxide and its role in ischaemic brain injury. Current molecular medicine, 4, 179-191.

278. KEYSER, R. J., LESAGE, S., BRICE, A., CARR, J. \& BARDIEN, S. 2010a. Assessing the prevalence of PINK1 genetic variants in South African patients diagnosed with early-and late-onset Parkinson's disease. Biochemical and biophysical research communications, 398 125-129. 
279. KEYSER, R. J., LOMBARD, D., VEIKONDIS, R., CARR, J. \& BARDIEN, S. 2010b. Analysis of exon dosage using MLPA in South African Parkinson's disease patients. Neurogenetics, $11,305-312$

280. KEYSER, R. J., OPPON, E., CARR, J. A. \& BARDIEN, S. 2011. Identification of Parkinson's disease candidate genes using CAESAR and screening of MAPT and SNCAIP in South African Parkinson's disease patients. Journal of Neural Transmission, 118, 889-897.

281. KEYSER, R. J., VAN DER MERWE, L., VENTER, M., KINNEAR, C., WARNICH, L., CARR, J. \& BARDIEN, S. 2009. Identification of a novel functional deletion variant in the 5'-UTR of the DJ-1 gene. BMC medical genetics, 10, 105.

282. Kilpatrick, B. S., MAgalhaes, J., BeAVAN, M. S., MCNEILl, A., GEGG, M. E., CLEETER, M. W., BLOOR-YOUNG, D., CHURCHILL, G. C., DUCHEN, M. R. \& SCHAPIRA A. H. 2015. Endoplasmic reticulum and lysosomal Ca 2+ stores are remodelled in GBA1-linked Parkinson disease patient fibroblasts. Cell Calcium, 59, 12-20.

283. KIM, H.-J., JEON, B., SONG, J., LEE, W.-W., PARK, H. \& SHIN, C.-W. 2016. Leukocyte glucocerebrosidase and $\beta$-hexosaminidase activity in sporadic and genetic Parkinson disease. Parkinsonism \& related disorders, 23, 99-101.

284. KIM, J., CINTRON, D., GRODEN, C., WIGGS, E., DAVIS, J., MISTRY, P., PASTORES, G., ZIMRAN, A., GOKER-ALPAN, O. \& SIDRANSKY, E. 2015. Gaucher disease and Parkinsonism: Clinical course and prognosis. Molecular Genetics and Metabolism, 114, S62.

285. KISS, P., SZOGYI, D., REGLODI, D., HORVATH, G., FARKAS, J., LUBICS, A., TAMAS, A., ATLASZ, T., SZABADFI, K. \& BABAI, N. 2009. Effects of perinatal asphyxia on the neurobehavioral and retinal development of newborn rats. Brain research, 1255, 42-50.

286. KISS, P., VADASZ, G., KISS-ILlES, B., HORVATH, G., TAMAS, A., REGLODI, D. \& KOPPAN, M. 2013. Environmental Enrichment Decreases Asphyxia-Induced Neurobehavioral Developmental Delay in Neonatal Rats. International journal of molecular sciences, 14, 22258-22273.

287. KITATANI, K., WADA, M., PERRY, D., USUI, T., SUN, Y., OBEID, L. M., YAEGASHI, N., GRABOWSKI, G. A. \& HANNUN, Y. A. 2015. Activation of p38 Mitogen-Activated Protein Kinase in Gaucher's Disease. PloS one, 10, e0136633.

288. KLAWITTER, V., MORALES, P., BUSTAMANTE, D., GOINY, M. \& HERRERA-MARSCHITZ, M. 2006. Plasticity of the central nervous system (CNS) following perinatal asphyxia: does nicotinamide provide neuroprotection? Amino Acids, 31, 377-384.

289. KLAWITTER, V., MORALES, P., BUSTAMANTE, D., GOMEZ-URQUIJO, S., HÖKFELT, T. \& HERRERA-MARSCHITZ, M. 2007. Plasticity of basal ganglia neurocircuitries following perinatal asphyxia: effect of nicotinamide. Experimental brain research, 180, 139-152.

290. KNEUSSEL, M. \& WAGNER, W. 2013. Myosin motors at neuronal synapses: drivers of membrane transport and actin dynamics. Nature Reviews Neuroscience, 14, 233-247.

291. KOHLhauser, C., MOSGOelleR, W., HOEGER, H., LUBEC, G. \& LUBEC, B. 1999. Cholinergic, monoaminergic and glutamatergic changes following perinatal asphyxia in the rat. Cell Mol Life Sci, 55, 1491-501.

292. KONG, S. M., CHAN, B. K., PARK, J.-S., HILL, K. J., AITKEN, J. B., COTTLE, L., FARGHAIAN, H., COLE, A. R., LAY, P. A. \& SUE, C. M. 2014. Parkinson's disease-linked human PARK9-ATP13A2 maintains zinc homeostasis and promotes $\alpha$-Synuclein externalization via exosomes. Human molecular genetics, 23, 2816-2833. 
293. KÖRSCHEN, H. G., YILDIZ, Y., RAJU, D. N., SCHONAUER, S., BÖNIGK, W., JANSEN, V., KREMMER, E., KAUPP, U. B. \& WACHTEN, D. 2013. The non-lysosomal $\beta$-glucosidase GBA2 is a non-integral membrane-associated protein at the endoplasmic reticulum (ER) and Golgi. Journal of Biological Chemistry, 288, 3381-3393.

294. KOSTOVIĆ, I. \& JOVANOV-MILOŠEVIĆ, N. The development of cerebral connections during the first 20-45 weeks' gestation. Seminars in Fetal and Neonatal Medicine, 2006. Elsevier, 415-422.

295. KOVACS, G. G. 2016. Molecular pathological classification of neurodegenerative diseases: turning towards precision medicine. International journal of molecular sciences, 17, 189.

296. KOVACSOVICS-BANKOWSKI, M., MAUCH, K., RABER, A., STREETER, P., DEANS, R., MAZIARZ, R. \& VAN'T HOF, W. 2008. Pre-clinical safety testing supporting clinical use of allogeneic multipotent adult progenitor cells. Cytotherapy, 10, 730-742.

297. KOZAKOV, D., BRENKE, R., COMEAU, S. R. \& VAJDA, S. 2006. PIPER: an FFTbased protein docking program with pairwise potentials. Proteins: Structure, Function, and Bioinformatics, 65, 392-406.

298. KOZLOWSKI, H., LUCZKOWSKI, M., REMELLI, M. \& VALENSIN, D. 2012. Copper, zinc and iron in neurodegenerative diseases (Alzheimer's, Parkinson's and prion diseases). Coordination Chemistry Reviews, 256, 2129-2141.

299. KRESOJEVIĆ, N., MIJAJLOVIĆ, M., PERIĆ, S., PAVLOVIĆ, A., SVETEL, M., JANKOVIĆ M., DOBRIČIĆ, V., NOVAKOVIĆ, I., LAKOČEVIĆ, M. B. \& KLEIN, C. 2013. Transcranial sonography in patients with Parkinson's disease with glucocerebrosidase mutations. Parkinsonism \& related disorders, 19, 431-435.

300. KUDO, T. \& TAKEDA, M. 2007. Neurodegenerative dementias involving aberrant protein aggregation. Handbook of Neurochemistry and Molecular Neurobiology. Springer.

301. KUMAR, K., RAMIREZ, A., GÖBEL, A., KRESOJEVIĆ, N., SVETEL, M., LOHMANN, K., M SUE, C., ROLFS, A., MAZZULLI, J. \& ALCALAY, R. 2013. Glucocerebrosidase mutations in a Serbian Parkinson's disease population. European Journal of Neurology, 20, 402-405.

302. KURTIS, M. M., RODRIGUEZ-BLAZQUEZ, C., MARTINEZ-MARTIN, P. \& GROUP, E. 2013. Relationship between sleep disorders and other non-motor symptoms in Parkinson's disease. Parkinsonism \& related disorders, 19, 1152-1155.

303. KUTMON, M., VAN IERSEL, M. P., BOHLER, A., KELDER, T., NUNES, N., PICO, A. R. \& EVELO, C. T. 2015. PathVisio 3: an extendable pathway analysis toolbox. PLoS computational biology, 11, e1004085.

304. LACHMANN, R. 2003. Miglustat. Oxford GlycoSciences Actelion. Current opinion in investigational drugs (London, England: 2000), 4, 472-479.

305. LACHMANN, R. H. 2011. Enzyme replacement therapy for lysosomal storage diseases. Current opinion in pediatrics, 23, 588-593.

306. LAHIRI, D. K. \& MALONEY, B. 2010. The "LEARn"(Latent Early-life Associated Regulation) model integrates environmental risk factors and the developmental basis of Alzheimer's disease, and proposes remedial steps. Experimental gerontology, 45, 291-296.

307. LAHIRI, D. K., MALONEY, B. \& ZAWIA, N. H. 2009. The LEARn model: an epigenetic explanation for idiopathic neurobiological diseases. Molecular psychiatry, 14, 992-1003. 
308. LAI, J. C., ROCHA-FERREIRA, E., EK, C. J., WANG, X., HAGBERG, H. \& MALLARD, C. 2017. Immune responses in perinatal brain injury. Brain, behavior, and immunity, 63, 210-223.

309. LANDWEHRMEYER, G. B., FITZER-ATTAS, C. J., GIULIANO, J. D., GONÇALVES, N., ANDERSON, K. E., CARDOSO, F., FERREIRA, J. J., MESTRE, T. A., STOUT, J. C. \& SAMPAIO, C. 2016. Data Analytics from Enroll-HD, a Global Clinical Research Platform for Huntington's Disease. Movement Disorders Clinical Practice 4, 212-224.

310. LANG, T. A. \& ALTMAN, D. G. 2014. Statistical analyses and methods in the published literature: The SAMPL guidelines. Guidelines for Reporting Health Research: A User's Manual (eds D Moher, DG Altman, K. F Schulz, I Simera and E Wager), John Wiley \& Sons, Ltd, Oxford, UK. doi, 10, 9781118715598.

311. LAPlANTE, F., BRAKE, W. G., CHEHAB, S. L. \& SUlLivAN, R. M. 2012. Sex differences in the effects of perinatal anoxia on dopamine function in rats. Neuroscience letters, 506, 8993.

312. LARSEN, S. D., Wilson, M. W., ABE, A., SHU, L., GEORGE, C. H., KIRCHHOFF, P., SHOWALTER, H. H., XIANG, J., KEEP, R. F. \& SHAYMAN, J. A. 2012. Property-based design of a glucosylceramide synthase inhibitor that reduces glucosylceramide in the brain. Journal of lipid research, 53, 282-291.

313. LAURENS, K. R., LUO, L., MATHESON, S. L., CARR, V. J., RAUDiNO, A., HARRIS, F. \& GREEN, M. J. 2015. Common or distinct pathways to psychosis? A systematic review of evidence from prospective studies for developmental risk factors and antecedents of the schizophrenia spectrum disorders and affective psychoses. BMC psychiatry, 15, 205.

314. LAVORGNA, A. \& HARHAJ, E. W. 2012. EBV LMP1: New and shared pathways to NF- $\kappa$ B activation. Proceedings of the National Academy of Sciences, 109, 2188-2189.

315. Lee, A. C., KOZUKi, N., Blencowe, H., Vos, T., Bahalim, A., Darmstadt, G. L., NIERMEYER, S., ELLIS, M., ROBERTSON, N. J. \& COUSENS, S. 2013. Intrapartumrelated neonatal encephalopathy incidence and impairment at regional and global levels for 2010 with trends from 1990. Pediatric research, 74, 50-72.

316. LEE, J.-M., WHEELER, V. C., CHAO, M. J., VONSATTEL, J. P. G., PINTO, R. M., LUCENTE, D., ABU-ElneEl, K., RAMOS, E. M., MYSORE, J. S. \& GILliS, T. 2015. Identification of genetic factors that modify clinical onset of Huntington's disease. Cell, 162, 516-526.

317. LENZ, J. D. \& LOBO, M. K. 2013. Optogenetic insights into striatal function and behavior. Behavioural brain research, 255, 44-54.

318. LESAGE, S. 2015. Genetics of Mendelian Forms of Parkinson's Disease. Movement Disorder Genetics. Springer.

319. LEVINE, Y. \& BOKSA, P. 2006. Maternal care is similar for rat pups subjected to birth hypoxia and for controls. Neuroreport, 17, 45-49.

320. LI, B., KRIShnAN, V. G., MORT, M. E., XIN, F., KAMATI, K. K., COOPER, D. N., MOONEY, S. D. \& RADIVOJAC, P. 2009. Automated inference of molecular mechanisms of disease from amino acid substitutions. Bioinformatics, 25, 2744-2750.

321. LI, Y., SEKINE, T., FUNAYAMA, M., LI, L., YOSHINO, H., NISHIOKA, K., TOMIYAMA, H. \& HATTORI, N. 2014. Clinicogenetic study of GBA mutations in patients with familial Parkinson's disease. Neurobiology of aging, 35, 935, e8. 
322. LILJEHOLM, M. \& O'DOHERTY, J. P. 2012. Contributions of the striatum to learning, motivation, and performance: an associative account. Trends in cognitive sciences, 16, 467475.

323. LINDSTRÖM, K., WINBLADH, B., HAGLUND, B. \& HJERN, A. 2007. Preterm infants as young adults: a Swedish national cohort study. Pediatrics, 120, 70-77.

324. LiOU, B., HAFFEY, W. D., GREIS, K. D. \& GRABOWSKI, G. A. 2014. The LIMP2/SCARB2 Binding Motif on Acid $\beta$-Glucosidase Basic and Applied Implications for Gaucher Disease and Associated Neurodegenerative Diseases. Journal of Biological Chemistry, 289, 30063-30074.

325. LIU, G., CHEN, M., MI, N., YANG, W., LI, X., WANG, P., YIN, N., LI, Y., YUE, F. \& CHAN, P. 2015. Increased oligomerization and phosphorylation of $\alpha$-synuclein are associated with decreased activity of glucocerebrosidase and protein phosphatase $2 \mathrm{~A}$ in aging monkey brains. Neurobiology of aging, 36, 2649-2659.

326. LIU, J. \& ZHANG, H. X. 2014. Significant study of population stratification, sensitivity analysis and trim and fill analyses on GBA mutation and parkinson's disease. American Journal of Medical Genetics Part B: Neuropsychiatric Genetics, 165, 96-102.

327. LOCHHEAD, J. J. \& THORNE, R. G. 2012. Intranasal delivery of biologics to the central nervous system. Advanced drug delivery reviews, 64, 614-628.

328. LOGITHARAJAH, P., RUTherForD, M. A. \& COWAN, F. M. 2009. Hypoxic-ischemic encephalopathy in preterm infants: antecedent factors, brain imaging, and outcome. Pediatric research, 66, 222-229.

329. LOIDL, C., HERRERA-MARSChITZ, M., ANDERSSON, K., YOU, Z.-B., GOINY, M., O'CONNOR, W., SILVEIRA, R., RAWAL, R., BJELKE, B. \& CHEN, Y. 1994. Longterm effects of perinatal asphyxia on basal ganglia neurotransmitter systems studied with microdialysis in rat. Neuroscience letters, 175, 9-12.

330. LOIDL, C. F., CAPANI, F., LÓPEZ-COSTA, J., SELVÍN-TESTA, A., LÓPEZ, E. M. \& PECCI-SAAVEDRA, J. 1997. Long term changes in NADPH-diaphorase reactivity in striatal and cortical neurons following experimental perinatal asphyxia: neuroprotective effects of hypothermia. International journal of neuroscience, 89, 1-14.

331. LOIDL, C. F., DE VENTE, J., VAN ITTERSUM, M. M., VAN DIJK, E. H., VLeS, J. S., STEINBUSCH, H. W. \& BLANCO, C. E. 1998a. Hypothermia during or after severe perinatal asphyxia prevents increase in cyclic GMP-related nitric oxide levels in the newborn rat striatum. Brain research, 791, 303-307.

332. LOIDL, C. F., DE Vente, J., VAn ITTERSum, M. M., VAN DiJK, E. H., VLes, J. S., STEINBUSCH, H. W. \& BLANCO, C. E. 1998b. Hypothermia during or after severe perinatal asphyxia prevents increase in cyclic GMP-related nitric oxide levels in the newborn rat striatum. Brain Research, 791, 303-7.

333. LOIDL, C. F., GAVILANES, A. D., VAN DIJK, E. H., VREUls, W., BLOKLAND, A. VLES, J. S., STEINBUSCH, H. W. \& BLANCO, C. E. 2000a. Effects of hypothermia and gender on survival and behavior after perinatal asphyxia in rats. Physiology \& behavior, 68 , 263-269.

334. LOIDL, C. F., GAVILANES, A. W., VAN DIJK, E. H., VREUlS, W., BLOKLAND, A., VLES, J. S., STEINBUSCH, H. W. \& BLANCO, C. E. 2000b. Effects of hypothermia and gender on survival and behavior after perinatal asphyxia in rats. Physiology \& Behavior, 68, 263-9. 
335. LOPEZ-FIGUEROA, M. O., CAAMANO, C., MORANO, M. I., RONN, L. C., AKIL, H. \& WATSON, S. J. 2000. Direct evidence of nitric oxide presence within mitochondria. Biochem Biophys Res Commun, 272, 129-33

336. LOWE, X. R., LU, X., MARCHETTI, F. \& WYROBEK, A. J. 2007. The expression of Troponin $\mathrm{T} 1$ gene is induced by ketamine in adult mouse brain. Brain research, 1174, 7-17.

337. LubeC, B., CHIAPPE-GUTIERREZ, M., HOEGER, H., KITZMUELLER, E. \& LUBEC, G. 2000. Glucose transporters, hexokinase, and phosphofructokinase in brain of rats with perinatal asphyxia. Pediatric research, 47, 84-84.

338. LUBEC, B., KOZLOV, A. V., KRAPFENBAUER, K., BERGER, A., HOEGER, H., HERRERAMARSCHITZ, M., NOHL, H., KOECK, T. \& LUBEC, G. 1999. Nitric oxide and nitric oxide synthase in the early phase of perinatal asphyxia of the rat. Neuroscience, 93, 1017-23.

339. LUO, T. WU, J., KABADI, S.V., SABIRZHANOV, B. GUANCIALE, K., HANSCOM, M. 2013. Propofol limits microglial activation after experimental brain trauma through inhibition of nicotinamide adenine dinucleotide phosphate oxidase. Anesthesiology, 119, 1370-1388.

340. MACASKILL, M. R., GRAHAM, C. F., PITCHER, T. L., MYALL, D. J., LIVINGSTON, L., VAN STOCKUM, S., DALRYMPLE-ALFORD, J. C. \& ANDERSON, T. J. 2012. The influence of motor and cognitive impairment upon visually-guided saccades in Parkinson's disease. Neuropsychologia, 50, 3338-3347.

341. MACHACZKA, M., HAST, R., DAHLMAN, I., LERNER, R., KLIMKOWSKA, M., ENGVALL, M. \& HÄGGLUND, H. 2012. Substrate reduction therapy with miglustat for type 1 Gaucher disease: a retrospective analysis from a single institution. Upsala journal of medical sciences, 117, 28-34.

342. MACHACZKA, M. \& KLIMKOWSKA, M. 2014. Novel heterozygous c. 798C > G and c $1040 \mathrm{~T}>\mathrm{G}$ mutations in the GBA1 gene are associated with a severe phenotype of Gaucher disease type 1 . Annals of hematology, 1-3.

343. MACONACHIE, G. D., GOTTLOB, I. \& MCLEAN, R. J. 2013. Risk factors and genetics in common comitant strabismus: a systematic review of the literature. JAMA ophthalmology, $131,1179-1186$

344. MAeGAWA, G. H., TROPAK, M. B., BUtTneR, J. D., RIGAT, B. A., FUlleR, M., PANDIT, D., TANG, L., KORNHABER, G. J., HAMURO, Y. \& CLARKE, J. T. 2009. Identification and characterization of ambroxol as an enzyme enhancement agent for Gaucher disease. Journal of Biological Chemistry, 284, 23502-23516.

345. MALEC-LITWINOWICZ, M., RUDZIŃSKA, M., SZUBIGA, M., MICHALSKI, M., TOMASZEWSKI, T. \& SZCZUDLIK, A. 2014. Cognitive impairment in carriers of glucocerebrosidase gene mutation in Parkinson disease patients. Neurologia i neurochirurgia polska, 48, 258-261.

346. MALini, E., GROSSI, S., DEGANUTO, M., ROSANO, C., PARINI, R., DOMINISINI, S., CARIATI, R., ZAMPIERI, S., BEMBI, B. \& FILOCAMO, M. 2014. Functional analysis of 11 novel GBA alleles. European Journal of Human Genetics, 22, 511-516.

347. MANIWANG, E., TAYEBI, N. \& SIDRANSKY, E. 2013. Is Parkinson disease associated with lysosomal integral membrane protein type-2?: Challenges in interpreting association data. Molecular genetics and metabolism, 108, 269-271.

348. MARRIOTT, A. L., ROJAS-MANCILLA, E., MORALES, P., HERRERA-MARSCHITZ, M. \& TASKER, R. A. 2015. Models of progressive neurological dysfunction originating early in life. Progress in neurobiology, 155, 2-20. 
349. MATA, I. F., LEVERENZ, J. B., WEINTRAUB, D., TROJANOWSKI, J. Q., CHENPLOTKIN, A., DEERLIN, V. M., RITZ, B., RAUSCH, R., FACTOR, S. A. \& WOODSIVERIO, C. 2015. GBA Variants are associated with a distinct pattern of cognitive deficits in Parkinson's disease. Movement Disorders, 31, 95-102.

350. MATHESON, S. L., SHEPHERD, A. M., LAURENS, K. R. \& CARR, V. J. 2011. A systematic meta-review grading the evidence for non-genetic risk factors and putative antecedents of schizophrenia. Schizophrenia research, 133, 133-142.

351. MATtoŠOvÁ, S., Chandoga, J., HLAVATÁ, A., SAligOvÁ, J. \& MACEKOVÁ, D. 2015. Spectrum of GBA mutations in patients with Gaucher disease from Slovakia: identification of five novel mutations. The Israel Medical Association journal: IMAJ, 17, 166-170.

352. MAZZULLI, J. R., XU, Y.-H., SUN, Y., KNIGHT, A. L., MCLEAN, P. J., CALDWELL, G. A., SIDRANSKY, E., GRABOWSKI, G. A. \& KRAINC, D. 2011. Gaucher disease glucocerebrosidase and $\alpha$-synuclein form a bidirectional pathogenic loop in synucleinopathies. Cell, 146, 37-52.

353. MCNEIll, A., DURAn, R., PROUKAKIS, C., BRAS, J., HUGHES, D., MEHTA, A., HARDY, J., WOOD, N. W. \& SCHAPIRA, A. H. 2012. Hyposmia and cognitive impairment in Gaucher disease patients and carriers. Movement Disorders, 27, 526-532.

354. MCNEILl, A., MAGAlHAES, J., Shen, C., CHAU, K.-Y., HUGHES, D., MEHTA, A., FOLTYNIE, T., COOPER, J. M., ABRAMOV, A. Y. \& GEGG, M. 2014. Ambroxol improves lysosomal biochemistry in glucocerebrosidase mutation-linked Parkinson disease cells. Brain, $137,1481-1495$

355. MCNEILL, A., ROBERTI, G., LASCARATOS, G., HUGHES, D., MEHTA, A., GARWAYHEATH, D. F. \& SCHAPIRA, A. H. 2013a. Retinal thinning in Gaucher disease patients and carriers: Results of a pilot study. Molecular genetics and metabolism, 109, 221-223.

356. MCNEILl, A., WU, R.-M., TZEN, K.-Y., AGUiAR, P. C., ARBElO, J. M., BARONE, P., BHATIA, K., BARSOTTINI, O., BONIFATI, V. \& BOSTANTJOPOULOU, S. 2013b. Dopaminergic neuronal imaging in genetic Parkinson's disease: insights into pathogenesis. PloS one, 8, e69190.

357. MCQUILLEN, P. S. \& FERRIERO, D. M. 2004. Selective vulnerability in the developing central nervous system. Pediatric neurology, 30, 227-235.

358. MEDRANO-ENGAY, B., IRUN, P., GERVAS-ARRUGA, J., ANDRADE-CAMPOS, M., ANDREU, V., ALFONSO, P., POCOVI, M. \& GIRALDO, P. 2014. Iron homeostasis and infIammatory biomarker analysis in patients with type 1 Gaucher disease. Blood Cells, Molecules, and Diseases, 53, 171-175

359. METTA, V., LOGISHETTY, K., MARTINEZ-MARTIN, P., GAGE, H. M., SCHARTAU, P., KALUARACHCHI, T., MARTIN, A., ODIN, P., BARONE, P. \& STOCCHI, F. 2011. The possible clinical predictors of fatigue in Parkinson's disease: a study of 135 patients as part of international nonmotor scale validation project. Parkinson's Disease, 2011.

360. MIDDLETON, F. A. \& STRICK, P. L. 2000. Basal ganglia and cerebellar loops: motor and cognitive circuits. Brain Res Brain Res Rev, 31, 236-50.

361. MielKe, M. M., MAETZLER, W., HAUGHEY, N. J., BANDARU, V. V., SAVICA, R., DEUSCHLE, C., GASSER, T., HAUSER, A.-K., GRÄBER-SULTAN, S. \& SCHLEICHER, E. 2013. Plasma Ceramide and Glucosylceramide Metabolism Is Altered in Sporadic Parkinson's Disease and Associated with Cognitive Impairment: A Pilot Study. PloS one, 8, e73094.

362. MISTRY, P. K., LIU, J., SUN, L., CHUANG, W.-L., YUEN, T., YANG, R., LU, P., ZHANG, K., LI, J. \& KEUTZER, J. 2014. Glucocerebrosidase 2 gene deletion rescues type 1 Gaucher disease. Proceedings of the National Academy of Sciences, 111, 4934-4939. 
363. MitSUi, J., MATSUKAWA, T., SASAKI, H., YABE, I., MATSUSHIMA, M., DÜRR, A., BRICE, A., TAKASHIMA, H., KIKUCHI, A. \& AOKI, M. 2015. Variants associated with Gaucher disease in multiple system atrophy. Annals of Clinical and Translational Neurology, $2,417-426$.

364. MO, C., HANNAN, A. J. \& RENOIR, T. 2015. Environmental factors as modulators of neurodegeneration: Insights from gene-environment interactions in Huntington's disease. Neuroscience \& Biobehavioral Reviews, 52, 178-192.

365. MOlero, A. E., ARTEAGA-BRACHO, E. E., CHEN, C. H., GUlinello, M., WiNCHESTER, M. L., PICHAMOORTHY, N., GOKHAN, S., KHODAKHAH, K. \& MEHLER, M. F. 2016. Selective expression of mutant huntingtin during development recapitulates characteristic features of Huntington's disease. Proceedings of the National Academy of Sciences, 201603871.

366. MORALES, P., BUSTAMANTE, D., ESPINA-MARCHANT, P., NEIRA-PEÑA, T., GUTIÉRREZHERNÁNDEZ, M. A., ALLENDE-CASTRO, C. \& ROJAS-MANCILlA, E. 2011. Pathophysiology of perinatal asphyxia: can we predict and improve individual outcomes? EPMA Journal, 2, 211.

367. MORALES, P., FIEDLER, J., ANDRES, S., BERRIOS, C., HUAIQUIN, P., BUSTAMANTE, D., CARDENAS, S., PARRA, E. \& HERRERA-MARSCHITZ, M. 2008. Plasticity of hippocampus following perinatal asphyxia: effects on postnatal apoptosis and neurogenesis. Journal of neuroscience research, 86, 2650-2662.

368. MORALES, P., HUAIQUIN, P., BUSTAMANTE, D., FIEDLER, J. \& HERRERA-MARSCHITZ, M. 2007. Perinatal asphyxia induces neurogenesis in hippocampus: an organotypic culture study. Neurotoxicity research, 12, 81-84.

369. MORAlES, P., SIMOLA, N., BUSTAMANTE, D., LISBOA, F., FIEDLER, J., GEBICKEHAERTER, P. J., MORELLI, M., TASKER, R. A. \& HERRERA-MARSCHITZ, M. 2010. Nicotinamide prevents the long-term effects of perinatal asphyxia on apoptosis, non-spatial working memory and anxiety in rats. Experimental brain research, 202, 1-14.

370. MORAR, B. \& LANE, A. B. 1996. The molecular characterization of Gaucher disease in South Africa. Clinical genetics, 50, 78-84.

371. MORENO-RAMOS, T., BENITO-LEÓN, J., VILLAREJO, A. \& BERMEJO-PAREJA, F. 2012. Retinal nerve fiber layer thinning in dementia associated with Parkinson's disease, dementia with Lewy bodies, and Alzheimer's disease. Journal of Alzheimer's disease: JAD, $34,659-664$.

372. MOSTER, D., LIE, R. T. \& MARKESTAD, T. 2008. Long-term medical and social consequences of preterm birth. New England Journal of Medicine, 359, 262-273.

373. MOUSSAUd, S., JONES, D. R., MOUSSAUd-LAmOdièRE, E. L., DELENCLOS, M., ROSS, O. A. \& MCLEAN, P. J. 2014. Alpha-synuclein and tau: teammates in neurodegeneration? Molecular neurodegeneration, 9, 43.

374. MUELlER-BURKE, D., KOEHLER, R. C. \& MARTIN, L. J. 2008. Rapid NMDA receptor phosphorylation and oxidative stress precede striatal neurodegeneration after hypoxic ischemia in newborn piglets and are attenuated with hypothermia. International Journal of Developmental Neuroscience, 26, 67-76.

375. MUELLER, M., OPPLIGER, B., JOERGER-MESSERLI, M., REINHART, U., BARNEA, E., PAIDAS, M., KRAMER, B. W., SURBEK, D. V. \& SCHOEBERLEIN, A. 2017. Wharton's Jelly Mesenchymal Stem Cells Protect the Immature Brain in Rats and Modulate Cell Fate. Stem cells and development, 26, 239-248. 
376. MUÑIZ, J., ROMERO, J., HOLUBIEC, M., BARRETO, G., GONZÁlEZ, J., SAINTMARTIN, M., BLANCO, E., CAVICCHIA, J. C., CASTILLA, R. \& CAPANI, F. 2014. Neuroprotective effects of hypothermia on synaptic actin cytoskeletal changes induced by perinatal asphyxia. Brain research, 1563, 81-90.

377. MURPHY, E. P. \& CREAN, D. 2015. Molecular interactions between NR4A orphan nuclear receptors and NF- $\kappa \mathrm{B}$ are required for appropriate inflammatory responses and immune cell homeostasis. Biomolecules, 5, 1302-1318.

378. MURPHY, K. E., GYSBERS, A. M., ABBOTT, S. K., TAYEBI, N., KIM, W. S., SIDRANSKY, E., COOPER, A., GARNER, B. \& HALLIDAY, G. M. 2014. Reduced glucocerebrosidase is associated with increased $\alpha$-synuclein in sporadic Parkinson's disease. Brain, 137, 834-848.

379. MWANIKI, M. K., ATIENO, M., LAWN, J. E. \& NEWTON, C. R. 2012. Long-term neurodevelopmental outcomes after intrauterine and neonatal insults: a systematic review. The Lancet, 379, 445-452.

380. NAKAGAWA, S., KUMIN, S., SACHS, G. \& NITOWSKY, H. M. 1982. Heterozygote detection of type I Gaucher disease using blood platelets. Clinica Chimica Acta, 118, 99-107.

381. NALlS, M. A., DURAN, R., LOPEZ, G., KURZAWA-AKANBI, M., MCKEITH, I. G., CHINNERY, P. F., MORRIS, C. M., THEUNS, J., CROSIERS, D. \& CRAS, P. 2013. A multicenter study of glucocerebrosidase mutations in dementia with Lewy bodies. JAMA neurology, 70, 727-735.

382. NALls, M. A., ESCOTT-PRiCE, V., Williams, N. M., LUBBE, S., KELLER, M. F., MORRIS, H. R. \& SINGLETON, A. B. 2015. Genetic risk and age in Parkinson's disease: Continuum not stratum. Movement Disorders, 30, 850-854.

383. NARITA, A., SHIRAI, K., KUBOTA, N., TAKAYAMA, R., TAKAHASHI, Y., ONUKI, T., NUMAKURA, C., KATO, M., HAMADA, Y. \& SAKAI, N. 2014. Abnormal pupillary light reflex with chromatic pupillometry in Gaucher disease. Annals of clinical and translational neurology, 1, 135-140.

384. NEIRA-PEÑA, T., ESPINA-MARCHANT, P., ROJAS-MANCILLA, E., ESMAR, D., KRAUS, C., MUNOZ, V., PEREZ, R., RIVERA, B., BUSTAMANTE, D. \& VALDES, J. 2014. Molecular, cellular, and behavioural effects produced by perinatal asphyxia: protection by poly (ADP-ribose) polymerase 1 (PARP-1) inhibition. Handbook of Neurotoxicity. Springer.

385. NEIRA-PEÑA, T., ROJAS-MANCILLA, E., MUNOZ-VIO, V., PEREZ, R., GUTIERREZHERNANDEZ, M., BUSTAMANTE, D., MORALES, P., HERMOSO, M., GEBICKE-HAERTER, P. \& HERRERA-MARSCHITZ, M. 2015. Perinatal Asphyxia Leads to PARP-1 Overactivity, p65 Translocation, IL-1 $\beta$ and TNF- $\alpha$ Overexpression, and Apoptotic-Like Cell Death in Mesencephalon of Neonatal Rats: Prevention by Systemic Neonatal Nicotinamide Administration. Neurotoxicity research, 27, 453-465.

386. NELSON, P. T., GREENBERG, S. G. \& SAPER, C. B. 1994. Neurofibrillary tangles in the cerebral cortex of sheep. Neuroscience letters, 170, 187-190.

387. NICHOLS, W., PANKRATZ, N., MAREK, D., PAUCIULO, M., ELSAESSER, V., HALTER C., RUDOLPH, A., WOJCIESZEK, J., PFEIFFER, R. \& FOROUD, T. 2009. Mutations in GBA are associated with familial Parkinson disease susceptibility and age at onset. Neurology, $72,310-316$

388. NIJBOER, C. H., HEIJNEN, C. J., GROENENDAAL, F., MAY, M. J., VAN BEL, F. \& KAVELAARS, A. 2008. A dual role of the NF- $\kappa$ B pathway in neonatal hypoxic-ischemic brain damage. Stroke, 39, 2578-2586. 
389. NIKIFOROU, M., WILLBURGER, C., DE JONG, A. E., KLOOSTERBOER, N., JELLEMA, R. K., OPHELDERS, D. R., STEINBUSCH, H. W., KRAMER, B. W. \& WOLFS, T. G. 2016. Global Hypoxia-Ischemia Induced Inflammation and Structural Changes in the Preterm Ovine Gut Which Were Not Ameliorated by Mesenchymal Stem Cell Treatment. Molecular Medicine, 22, 244.

390. NISHIOKA, K., ROSS, O. A., ISHII, K., KACHERGUS, J. M., ISHIWATA, K., KITAGAWA M., KONO, S., OBI, T., MIZOGUCHI, K. \& INOUE, Y. 2009. Expanding the clinical phenotype of SNCA duplication carriers. Movement disorders, 24, 1811-1819.

391. NOELKER, C., LU, L., HÖLlERHAGE, M., VULINOVIC, F., STURN, A., ROSCHER, R., HÖGLINGER, G. U., HIRSCH, E. C., OERTEL, W. H. \& ALVAREZ-FISCHER, D. 2015. Glucocerebrosidase deficiency and mitochondrial impairment in experimental Parkinson disease. Journal of the Neurological Sciences.

392. NOYCE, A., MENCACCI, N., SCHRAG, A., GIOVANNONI, G., LEES, A. \& HARDY, J. 2014. GBA1 and LRRK2 mutations in higher and lower risk subjects from the PREDICT-PD project. Movement Disorders 29,58-59

393. NUMERATO, D., FATTORE, G., TEDIOSI, F., ZANINI, R., PELTOLA, M., BANKS, H., MIHAliCZA, P., LEHTONEN, L., SVERÉUS, S. \& HEIJINK, R. 2015. Mortality and length of stay of very low birth weight and very preterm infants: a EuroHOPE study. PloS one, 10, e0131685.

394. NUZHNYI, E., EMELYANOV, A., BOUKINA, T., USENKO, T., YAKIMOVSKII, A., ZAKHAROVA, E. \& PCHELINA, S. 2015. Plasma Oligomeric Alpha-Synuclein Is Associated With Glucocerebrosidase Activity in Gaucher Disease. Movement Disorders, 30, 989-991.

395. ODD, D. \& WHITELAW, A. 2013. Neurological outcome after perinatal asphyxia at term. Neonatal Neural Rescue: A Clinical Guide, 1

396. OKUMUS, N., TURKYILMAZ, C., ONAL, E. E., ATALAY, Y., SERDAROGLU, A., ELBEG, S., KOC, E., DEDA, G., CANSU, A. \& GUNDUZ, B. 2008. Tau and S100B proteins as biochemical markers of bilirubin-induced neurotoxicity in term neonates. Pediatric neurology, $39,245-252$.

397. OLDREIVE, C. E., GAYNOR, S. \& DOHERTY, G. H. 2012. Effects of nitric oxide on the survival and neuritogenesis of cerebellar Purkinje neurons. Journal of Molecular Neuroscience, $46,336-342$.

398. OLIVEIRA, J., JEKABSONS, M. B., CHEN, S., LIN, A., REGO, A. C., GONCALVES, J., ELLERBY, L. M. \& NICHOLLS, D. G. 2007. Mitochondrial dysfunction in Huntington's disease: the bioenergetics of isolated and in situ mitochondria from transgenic mice. Journal of neurochemistry, 101, 241-249.

399. OLIVEROS, J. 2015. VENNY. An interactive tool for comparing lists with Venn Diagrams. 2007.

400. OPHELDERS, D. R., WOLFS, T. G., JELLEMA, R. K., ZWANENBURG, A., ANDRIESSEN, P., DELHAAS, T., LUDWIG, A.-K., RADTKE, S., PETERS, V. \& JANSSEN, L. 2016. Mesenchymal Stromal Cell-Derived Extracellular Vesicles Protect the Fetal Brain After HypoxiaIschemia. Stem cells translational medicine, 5, 754-763.

401. ORTEGA, R. A., TORRES, P. A., SWAN, M., NICHOLS, W., BOSCHUNG, S., RAYMOND, D., BARRETT, M. J., JOHANNES, B. A., SEVERT, L. \& SHANKER, V. 2016. Glucocerebrosidase enzyme activity in GBA mutation Parkinson's disease. Journal of Clinical Neuroscience, 28, 185-186. 
402. ORTH, M. \& NETWORK, E. H. S. D. 2010. Observing Huntington's disease: the European Huntington's disease network's REGISTRY. Journal of Neurology, Neurosurgery \& Psychiatry, 82, 1409-1412.

403. PAgidA, M. A., KOnStantinidou, A. E., TSEKOURA, E., MANGOURA, D., PATSOURIS, E. \& PANAYOTACOPOULOU, M. T. 2013. Vulnerability of the mesencephalic dopaminergic neurons of the human neonate to prolonged perinatal hypoxia: an immunohistochemical study of tyrosine hydroxylase expression in autopsy material. Journal of Neuropathology \& Experimental Neurology, 72, 337-350.

404. PANDEY, M. K., JABRE, N. A., XU, Y.-H., ZHANG, W., SETCHELL, K. D. \& GRABOWSKI, G. A. 2014. Gaucher disease: Chemotactic factors and immunological cell invasion in a mouse model. Molecular genetics and metabolism, 111, 163-171.

405. PANKRATZ, N., BEECHAM, G. W., DESTEFANO, A. L., DAWSON, T. M., DOHENY, K. F., FACTOR, S. A., HAMZA, T. H., HUNG, A. Y., HYMAN, B. T. \& IVINSON, A. J. 2012. Meta-analysis of Parkinson's Disease: Identification of a novel locus, RIT2. Annals of neurology, 71, 370-384.

406. PARENTI, G., ANDRIA, G. \& VALEnZANO, K. J. 2015. Pharmacological Chaperone Therapy: Preclinical Development, Clinical Translation, and Prospects for the Treatment of Lysosomal Storage Disorders. Molecular Therapy, 23, 1138-1148.

407. PAUlSEN, J., LANGBEHN, D., STOUT, J., AYlWARD, E., ROSS, C., NANCE, M., GUTTMAN, M., JOHNSON, S., MACDONALD, M. \& BEGLINGER, L. 2008. Detection of Huntington's disease decades before diagnosis: the Predict-HD study. Journal of Neurology, Neurosurgery \& Psychiatry, 79, 874-880.

408. PAYNE, T., KEATINGE, M., DA COSTA, M. \& BANDMANN, O. 2015. MPP+ IN A ZEBRAFISH MODEL OF GLUCOCEREBROSIDASE 1 DEFICIENCY. Journal of Neurology, Neurosurgery \& Psychiatry, 86, e4-e4.

409. PÉREZ-SeVEriano, F., EScAlante, B., Vergara, P., Ríos, C. \& SEgOvia, J. 2002. Age-dependent changes in nitric oxide synthase activity and protein expression in striata of mice transgenic for the Huntington's disease mutation. Brain research, 951, 36-42.

410. PERLMAN, J. M. 2006. Intervention strategies for neonatal hypoxic-ischemic cerebral injury. Clinical therapeutics, 28, 1353-1365.

411. PETERSON, R. L. \& PENnington, B. F. 2012. Developmental dyslexia. The Lancet, $379,1997-2007$.

412. PIERSON, C. R., FOLKERTH, R. D., BILLIARDS, S. S., TRACHTENBERG, F. L., DRINKWATER, M. E., VOLPE, J. J. \& KINNEY, H. C. 2007. Gray matter injury associated with periventricular leukomalacia in the premature infant. Acta neuropathologica, 114, 619-631.

413. PIMENTEL-COELHO, P. M. \& MENDEZ-OTERO, R. 2010. Cell therapy for neonatal hypoxic-ischemic encephalopathy. Stem cells and development, 19, 299-310.

414. PIÑA-CRESPO, J. C., SANZ-BLASCO, S. \& LIPTON, S. A. 2014. Concept of Excitotoxicity via Glutamate Receptors. Handbook of Neurotoxicity. Springer.

415. PISCOPO, P., BERNARDO, A., CALAMANDREI, G., VENEROSI, A., VALANZANO, A., BIANCHI, D., CONFALONI, A. \& MINGHETTI, L. 2008. Altered expression of cyclooxygenase2 , presenilins and oxygen radical scavenging enzymes in a rat model of global perinatal asphyxia. Experimental neurology, 209, 192-198.

416. POCKLINGTON, A. J., O'DONOVAN, M. \& OWEN, M. J. 2014. The synapse in schizophrenia. European Journal of Neuroscience, 39, 1059-1067. 
417. POLANCZYK, G., DE LIMA, M. S., HORTA, B. L., BIEDERMAN, J. \& ROHDE, L. A. 2007. The worldwide prevalence of ADHD: a systematic review and metaregression analysis. American journal of psychiatry, 164, 942-948.

418. POOLE, R. M. 2014. Eliglustat: first global approval. Drugs, 74, 1829-1836.

419. PRingsheim, T., JeTte, N., FRolkis, A. \& SteEves, T. D. 2014. The prevalence of Parkinson's disease: A systematic review and meta-analysis. Movement Disorders, 29, $1583-1590$

420. PRINGSheim, T., WILTSHIRE, K., DAY, L., DYKEMAN, J., STEEVES, T. \& JETTE, N. 2012. The incidence and prevalence of Huntington's disease: A systematic review and meta-analysis. Movement Disorders, 27, 1083-1091.

421. PUlkes, T., Choubtum, L., Chitphuk, S., Thakkinstian, A., PONGPAKdeE, S., KULKANTRAKORN, K., HANCHAIPHIBOOLKUL, S., TIAMKAO, S. \& BOONKONGCHUEN, P. 2014. Glucocerebrosidase mutations in Thai patients with Parkinson's disease. Parkinsonism \& related disorders, 20, 986-991.

422. RAHIM, A. A., WONG, A. M., HOEFER, K., BUCKLEY, S. M., MATTAR, C. N., CHENG, S. H., CHAN, J. K., COOPER, J. D. \& WADDINGTON, S. N. 2011. Intravenous administration of AAV2/9 to the fetal and neonatal mouse leads to differential targeting of CNS cell types and extensive transduction of the nervous system. The FASEB Journal, 25, 3505-3518.

423. RAN, C., WESTERLUND, M. O., RAMEZANI, M., GELLHAAR, S., XIANG, F., FARDELL, C., NISSBRANDT, H., SODERKVIST, P., WILlOWS, T. \& WILlOWS, T. 2014. Glucocerebrosidase (GBA) mutations in Parkinson's disease in Sweden and tissue expression of GBA mRNA. Movement Disorders, 29,60.

424. RANCILlaC, A., ROSSIER, J., GUILle, M., TONG, X.-K., GEOFFrOY, H., AMATORE, C., ARBAULT, S., HAMEL, E. \& CAULI, B. 2006. Glutamatergic control of microvascular tone by distinct GABA neurons in the cerebellum. The Journal of neuroscience, 26, 69977006 .

425. RAO, R., TRIVEDI, S., VESOUlis, Z., LIAO, S. M., SMYSER, C. D. \& MATHUR, A. M. 2016. Safety and Short-Term Outcomes of Therapeutic Hypothermia in Preterm Neonates 3435 Weeks Gestational Age with Hypoxic-Ischemic Encephalopathy. The Journal of Pediatrics, $183,37-42$

426. RAO, S., LIN, Z., DROBYShevsky, A., ChEN, L., JI, X., JI, H., YANG, Y., YU, L., DERRICK, M. \& SILVERMAN, R. B. 2011. Involvement of neuronal nitric oxide synthase in ongoing fetal brain injury following near-term rabbit hypoxia-ischemia. Developmental neuroscience, 33, 288-298.

427. RAWlins, M. D., WeXleR, N. S., WeXleR, A. R., TABRIZI, S. J., DOUGlas, I., EVANS, S. J. \& SMEETH, L. 2016. The Prevalence of Huntington's Disease. Neuroepidemiology, 46, 144-153.

428. RECZEK, D., SCHWAKE, M., SCHRÖDER, J., HUGHES, H., BLANZ, J., JIN, X., BRONDYK, W., VAN PATTEN, S., EDMUNDS, T. \& SAFTIG, P. 2007. LIMP-2 is a receptor for lysosomal mannose-6-phosphate-independent targeting of $\beta$-glucocerebrosidase. Cell, 131, 770-783.

429. REES, S., HARDING, R. \& WALKER, D. 2011. The biological basis of injury and neuroprotection in the fetal and neonatal brain. International Journal of Developmental Neuroscience, $29,551-563$.

430. RICHTER, F., FLEMING, S. M., WATSON, M., LEMESRE, V., PELLEGRINO, L., RANES, B., ZHU, C., MORTAZAVI, F., MULLIGAN, C. K. \& SIOSHANSI, P. C. 2014. A GCase chaperone improves motor function in a mouse model of synucleinopathy. Neurotherapeutics, $1-17$. 
431. ROBERTSON, N. J., TAN, S., GROENENDAAL, F., VAN BEL, F., JUUL, S. E., BENNET, L., DERRICK, M., BACK, S. A., VAlDEZ, R. C. \& NORTHINGTON, F. 2012. Which neuroprotective agents are ready for bench to bedside translation in the newborn infant? The Journal of pediatrics, 160, 544 .

432. ROCHA, E. M., SMITH, G. A., PARK, E., CAO, H., BROWN, E., HALLETT, P. \& ISACSON, O. 2015a. Progressive decline of glucocerebrosidase in aging and Parkinson's disease. Annals of Clinical and Translational Neurology, 2(4), 433-438.

433. ROCHA, E. M., SMITH, G. A., PARK, E., CAO, H., BROWN, E., HAYES, M. A., BEAGAN, J., MCLEAN, J. R., IZEN, S. C. \& PEREZ-TORRES, E. 2015b. Glucocerebrosidase gene therapy prevents $\alpha$-synucleinopathy of midbrain dopamine neurons. Neurobiology of disease, $82,495-503$.

434. RODRIGUES, F. B., ABREU, D., DAMÁSIO, J., GONCALVES, N., CORREIA-GUEDES, L., COELHO, M. \& FERREIRA, J. J. 2017a. Survival, mortality, causes and places of death in a European Huntington's disease prospective cohort. Movement Disorders Clinical Practice.

435. RODRIGUES, F. B., MESTRE, T., DUARTE, S. D., BARRA, M., COSTA, J., SAMPAIO, C. \& FERREIRA, J. J. [122] Therapeutic interventions for symptomatic treatment in Huntington's disease - a Cochrane review. In: CHDI, ed. CHDI 12th Annual HD Therapeutics Conference, 2017b St Julien, Malta.

436. RODRIGUEZ, J., SPECIAN, V., MALONEY, R., JOURD'HEUIL, D. \& FEELISCH, M 2005. Performance of diamino fluorophores for the localization of sources and targets of nitric oxide. Free Radical Biology and Medicine, 38, 356-368.

437. ROMANOS, M., WEISE, D., SCHLIESSER, M., LÖFFLER, J., CLASSEN, J. \& MEHLERWEX, C. 2010. Structural abnormality of the substantia nigra in children with attentiondeficit hyperactivity disorder. Journal of psychiatry \& neuroscience: JPN, 35, 55.

438. RON, I. \& HOROWITZ, M. 2005. ER retention and degradation as the molecular basis underlying Gaucher disease heterogeneity. Human molecular genetics, 14, 2387-2398.

439. RON, I., RAPAPORT, D. \& HOROWITZ, M. 2010. Interaction between parkin and mutant glucocerebrosidase variants: a possible link between Parkinson disease and Gaucher disease. Human molecular genetics, 19, 3771-3781.

440. ROSEnBloOM, B., BALWAni, M., BROnSTEIn, J. M., KOLODNY, E., SATHe, S., GWOSDOW, A. R., TAYLOR, J. S., COLE, J. A., ZIMRAN, A. \& WEINREB, N. J. 2011. The incidence of Parkinsonism in patients with type 1 Gaucher disease: data from the ICGG Gaucher Registry. Blood Cells, Molecules, and Diseases, 46, 95-102.

441. ROTHAUG, M., ZUNKE, F., MAZZULLI, J. R., SCHWEIZER, M., ALTMEPPEN, H. LÜLLMANN-RAUCH, R., KALLEMEIJN, W. W., GASPAR, P., AERTS, J. M. \& GLATZEL, M. 2014. LIMP-2 expression is critical for $\beta$-glucocerebrosidase activity and $\alpha$-synuclein clearance. Proceedings of the National Academy of Sciences, 111, 15573-15578.

442. ROYCHOWDHuRY, S., LUTHE, A., KeIlHOFF, G., WOLF, G. \& HORN, T. F. 2002. Oxidative stress in glial cultures: detection by DAF-2 fluorescence used as a tool to measure peroxynitrite rather than nitric oxide. Glia, 38, 103-14.

443. ROZENBERG, R., FOX, D., SOBREIRA, E. \& PEREIRA, L. 2006. Detection of 12 new mutations in Gaucher disease Brazilian patients. Blood Cells, Molecules, and Diseases, 37, 204-209. 
444. RÜB, U., VONSATTEL, J. P. G., HEINSEN, H. \& KORF, H.-W. 2015. Consistent and Widespread Degeneration of the Cerebellum in Huntington's Disease (HD). The Neuropathology of Huntington's Disease: Classical Findings, Recent Developments and Correlation to Functional Neuroanatomy. Springer.

445. RUDOLPH, U. \& ANTKOWIAK, B. 2004. Molecular and neuronal substrates for general anaesthetics. Nature Reviews Neuroscience, 5, 709-720.

446. SALAT, D., NOYCE, A. J., SCHRAG, A. \& TOLOSA, E. 2016. Challenges of modifying disease progression in prediagnostic Parkinson's disease. The Lancet Neurology, 15, 637-648.

447. SAMUELS, N., ELSTEIN, D., LEBEL, E., ZIMRAN, A. \& OBERBAUM, M. 2012. Acupuncture for symptoms of Gaucher disease. Complementary therapies in medicine, 20, 131-134.

448. SARACENO, G., AYAlA, M., BADORREY, M., HOLUBIEC, M., ROMERO, J., GALEANO, P., BARRETO, G., GIRALDEZ-ALVÁREZ, L., KÖLlIKER-FRES, R. \& COIRINI, H. 2012a. Effects of perinatal asphyxia on rat striatal cytoskeleton. Synapse, 66, 9-19.

449. SARACENO, G., CACERES, L., GUELMAN, L., CASTILlA, R., UDOVIN, L., ELlisMAN, M., BROCCO, M. \& CAPANI, F. 2016. Consequences of excessive plasticity in the hippocampus induced by perinatal asphyxia. Experimental Neurology, 286, 116-123.

450. SARACENO, G., CASTILlA, R., BARRETO, G., GONZALEZ, J., KÖLLIKER-FrERS, R. \& CAPANI, F. 2012b. Hippocampal dendritic spines modifications induced by perinatal asphyxia. Neural plasticity, 2012

451. SARACEnO, G. E., Bertolino, M. L. A., GAleAno, P., Romero, J. I., GARCiASEGURA, L. M. \& CAPANI, F. 2010. Estradiol therapy in adulthood reverses glial and neuronal alterations caused by perinatal asphyxia. Experimental neurology, 223, 615-622.

452. SARDi, S. P., ClARKE, J., VIEL, C., CHAN, M., TAMSETT, T. J., TRELEAVEN, C. M., BU, J., SWEET, L., PASSINI, M. A. \& DODGE, J. C. 2013. Augmenting CNS glucocerebrosidase activity as a therapeutic strategy for parkinsonism and other Gaucher-related synucleinopathies. Proceedings of the National Academy of Sciences, 110, 3537-3542.

453. SARNAT, H. B., AUER, R. N. \& FLORES-SARNAT, L. 2013. Synaptogenesis in the Fetal Corpus Striatum, Globus Pallidus, and Substantia Nigra Correlations With Striosomes of Graybiel and Dyskinesias in Premature Infants. Journal of child neurology, 28, 60-69.

454. SATO, C., MORGan, A., LANG, A. E., SAlEhi-RAD, S., KAWARAI, T., MENG, Y., RAY, P. N., FARRER, L. A., GEORGE-HYSLOP, S. \& ROGAEVA, E. 2005. Analysis of the glucocerebrosidase gene in Parkinson's disease. Movement disorders, 20, 367-370.

455. SCHAAF, J., MOL, B., ABU-HANNA, A. \& RAVELLI, A. 2011. Trends in preterm birth: singleton and multiple pregnancies in the Netherlands, 2000-2007. BJOG: An International Journal of Obstetrics \& Gynaecology, 118, 1196-1204.

456. SCHACHAR, R. 2014. Genetics of Attention Deficit Hyperactivity Disorder (ADHD): recent updates and future prospects. Current Developmental Disorders Reports, 1, 41-49.

457. SCHAPIRA, A. H. \& GEGG, M. E. 2013. Glucocerebrosidase in the pathogenesis and treatment of Parkinson disease. Proceedings of the National Academy of Sciences, 110, 3214-3215.

458. SCHEEPENS, A., WASSINK, G. \& BLANCO, C. E. 2003a. The effect of a global birth asphyxia on the ontogeny of BDNF and NGF protein expression in the juvenile brain. Developmental brain research, 140, 215-221.

459. SCHEEPENS, A., WASSINK, G., PIERSMA, M. J., VAN DE BERG, W. D. \& BLANCO, C. E. 2003b. A delayed increase in hippocampal proliferation following global asphyxia in the neonatal rat. Developmental brain research, 142, 67-76. 
460. SCHENCK, C. H., BOEVE, B. F. \& MAHOWALD, M. W. 2013. Delayed emergence of a parkinsonian disorder or dementia in $81 \%$ of older men initially diagnosed with idiopathic rapid eye movement sleep behavior disorder: a 16-year update on a previously reported series. Sleep medicine, 14, 744-748.

461. SCHIFFMANN, R., FITZGIBBON, E. J., HARRIS, C., DEVILE, C., DAVIES, E. H., ABEL, L., VAN SCHAIK, I. N., BENKO, W. S., TIMMONS, M. \& RIES, M. 2008. Randomized, controlled trial of miglustat in Gaucher's disease type 3. Annals of neurology, 64, 514-522.

462. SCHMID, S. P., SCHLEICHER, E. D., CEGAN, A., DEUSCHLE, C., BAUR, S., HAUSER, A. K., SYNOFZIK, M., SRULIJES, K., BROCKMANN, K. \& BERG, D. 2012. Cerebrospinal fluid fatty acids in glucocerebrosidase-associated Parkinson's disease. Movement Disorders, $27,288-293$.

463. SCHÖNDORF, D. C., AURELI, M., MCALLISTER, F. E., HINDLEY, C. J., MAYER, F., SCHMID, B., SARDI, S. P., VALSECCHI, M., HOFFMANN, S. \& SCHWARZ, L. K. 2014 iPSC-derived neurons from GBA1-associated Parkinson's disease patients show autophagic defects and impaired calcium homeostasis. Nature Communications, 5, 4028.

464. SCHREPFER, S., DEUSE, T., REICHENSPURNER, H., FISCHBEIN, M., ROBBINS, R. \& PELLETIER, M. Stem cell transplantation: the lung barrier. Transplantation proceedings, 2007. Elsevier, 573-576.

465. SCHWALLER, B., MEYER, M. \& SCHIFFMANN, S. 2002. 'New'functions for 'old'proteins: the role of the calcium-binding proteins calbindin D-28k, calretinin and parvalbumin, in cerebellar physiology. Studies with knockout mice. The Cerebellum, 1, 241-258.

466. Seehase, M., HOUthuizen, P., JellemA, R. K., COllins, J. J., BeKers, O., BREUER, J. \& KRAMER, B. W. 2013. Propofol administration to the fetal-maternal unit reduces cardiac injury in late-preterm lambs subjected to severe prenatal asphyxia and cardiac arrest. Pediatric research, 73, 427-434.

467. SEEHASE, M., JELLEMA, R., JENNEKENS, W., ZWANENBURG, A., ANDRIESSEN, P., GAVILANES, D. \& KRAMER, B. 2011. Fetal Cardiac Arrest Due to Asphyxia in Late Preterm Lambs: Propofol Mediates Neuroprotection for the Fetus when Administered During Emergency Caesarean Section and after Rescusitation. Pediatric Research, 70, 61-61.

468. SEEHASE, M., JENNEKENS, W., ZWANENBURG, A., ANDRIESSEN, P., COLLINS, J. J., KUYPERS, E., ZIMMERMANN, L. J., VLES, J. S., GAVILANES, A. W. \& KRAMER, B. W 2015. Propofol administration to the maternal-fetal unit improved fetal EEG and influenced cerebral apoptotic pathway in preterm lambs suffering from severe asphyxia. Molecular and Cellular Pediatrics, 2, 4 .

469. SEGARANE, B., LI, A., PAUDEL, R., SCHOlZ, S., NEUMANN, J., LEES, A., REVESZ, T., HARDY, J., MATHIAS, C. \& WOOD, N. 2009. Glucocerebrosidase mutations in 108 neuropathologically confirmed cases of multiple system atrophy. Neurology, 72, 1185-1186.

470. SEIDL, R., STÖCKLER-IPSIROGLU, S., ROLINSKI, B., KOHLHAUSER, C., HERKNER, K. R., LUBEC, B. \& LUBEC, G. 2000. Energy metabolism in graded perinatal asphyxia of the rat. Life sciences, 67, 421-435.

471. SEIDOVA, S.-F., KOTLIAR, K., FOERGER, F., KLOPFER, M. \& LANZL, I. 2009. Functional retinal changes in Gaucher disease. Documenta ophthalmologica, 118, 151-154.

472. SEIFAN, A., SCHELKE, M., OBENG-ADUASARE, Y. \& ISAACSON, R. 2015. Early life epidemiology of Alzheimer's disease-a critical review. Neuroepidemiology, 45, 237-254.

473. SEMPLE, B. D., BLOMGREN, K., GIMLIN, K., FERRIERO, D. M. \& NOBLE-HAEUSSLEIN, L. J. 2013. Brain development in rodents and humans: identifying benchmarks of maturation and vulnerability to injury across species. Progress in neurobiology, 106, 1-16. 
474. SHAH, D. K., ANDERSON, P. J., CARLIN, J. B., PAVLOVIC, M., HOWARD, K., THOMPSON, D. K., WARFIELD, S. K. \& INDER, T. E. 2006. Reduction in cerebellar volumes in preterm infants: relationship to white matter injury and neurodevelopment at two years of age. Pediatric research, 60, 97-102.

475. SHEMESH, E., DEROMA, L., BEMBI, B., DEEGAN, P., HOLLAK, C., WEINREB, N. J. \& COX, T. M. 2015. Enzyme replacement and substrate reduction therapy for Gaucher disease. The Cochrane Library, 3.

476. SHIM, S.-Y. \& KIM, H.-S. 2013. Oxidative stress and the antioxidant enzyme system in the developing brain. Korean journal of pediatrics, 56, 107-111.

477. SIAN-HÜLSMANN, J., MANDEL, S., YOUdiM, M. B. \& RIEDERER, P. 2011. The relevance of iron in the pathogenesis of Parkinson's disease. Journal of neurochemistry, 118, 939-957.

478. SIDRANSKY, E. \& LOPEZ, G. 2012. The link between the GBA gene and parkinsonism. The Lancet Neurology, 11, 986-998.

479. SIDRANSKY, E., NAlls, M. A., AASly, J. O., AhARON-PERETZ, J., ANNESI, G., BARBOSA, E. R., BAR-SHIRA, A., BERG, D., BRAS, J. \& BRICE, A. 2009. Multicenter analysis of glucocerebrosidase mutations in Parkinson's disease. New England Journal of Medicine, 361, 1651-1661.

480. SIEBERT, M., WESTBROEK, W., CHEN, Y.-C., MOAVEN, N., LI, Y., SARAIVA-PEREIRA, M. L., MARTIN, S. \& SIDRANSKY, E. 2014a. MiRNAs and glucocerebrosidase: lessons from miRNA mimic screening. Molecular Genetics and Metabolism, 111.

481. SiEBERT, M., WESTBROEK, W., CHEN, Y.-C., MOAVEN, N., LI, Y., VELAYATI, A., SARAIVA-PEREIRA, M. L., MARTIN, S. E. \& SIDRANSKY, E. 2014b. Identification of miRNAs that modulate glucocerebrosidase activity in Gaucher disease cells. RNA biology, 01-14.

482. Simola, N., BUSTAMANTE, D., PinNA, A., PONTIS, S., MORAleS, P., MORELli, M. \& HERRERA-MARSCHITZ, M. 2008. Acute perinatal asphyxia impairs non-spatial memory and alters motor coordination in adult male rats. Experimental brain research, 185, 595-601.

483. SIMON-TOV, S., DINUR, T., GILADI, N., BAR-SHIRA, A., ZELIS, M., ZIMRAN, A. \& ELSTEIN, D. 2015. Color Discrimination in Patients with Gaucher Disease and Parkinson Disease. Journal of Parkinson's Disease, 1-7.

484. SINGLETON, A. B., FARRER, M. J. \& BONIFATI, V. 2013. The genetics of Parkinson's disease: progress and therapeutic implications. Movement Disorders, 28, 14-23.

485. SINNER, B., BECKE, K. \& ENGELHARD, K. 2014. General anaesthetics and the developing brain: an overview. Anaesthesia, 69, 1009-1022.

486. SMIT, A. L., SEEHASE, M., STOKROOS, R. J., JELLEMA, R. K., FELIPE, L., CHENAULT, M. N., ANTEUNIS, L. J., KREMER, B. \& KRAMER, B. W. 2013. Functional impairment of the auditory pathway after perinatal asphyxia and the short-term effect of perinatal propofol anesthesia in lambs. Pediatric research, 74, 34-38.

487. SMITH, T. F., SCHMIDT-KASTNER, R., MCGEARY, J. E., KACZOROWSKI, J. A. \& KNOPIK, V. S. 2016. Pre-and Perinatal Ischemia-Hypoxia, the Ischemia-Hypoxia Response Pathway, and ADHD Risk. Behavior genetics, 1-11.

488. SMOOT, M. E., ONO, K., RUSCHEINSKI, J., WANG, P.-L. \& IDEKER, T. 2010. Cytoscape 2.8: new features for data integration and network visualization. Bioinformatics, 27, 431-432. 
489. SMYTH, G. 2005. Limma: linear models for microarray data. Bioinformatics and computational biology solutions using $\mathrm{R}$ and Bioconductor, 397-420.

490. SOUZA, S. K., MARTINS, T. L., FERREIRA, G. D., VINAGRE, A. S., DA SILVA, R. S. M. \& FRIZZO, M. E. 2013. Metabolic effects of perinatal asphyxia in the rat cerebral cortex. Metabolic brain disease, 28, 25-32.

491. SPARNAAIJ, M., CHEDRAUI, P., LIEM, K. H., ESCOBAR, G. S., ESPINOZA-CAICEDO, J., KRAMER, B. W., VLES, J. S. \& GAVILANES, A. W. 2016. Fetal asphyctic preconditioning in rats results in a preserved placental inflammatory phenotype at birth. Placenta, $38,84-92$.

492. SPATARO, N., CAlafell, F., CERVERA-CARles, L., CASAls, F., PAGONABARRAGA, J., PASCUAL-SEDANO, B., CAMPOLONGO, A., KULISEVSKY, J., LLEÓ, A. \& NAVARRO, A. 2015. Mendelian genes for Parkinson's disease contribute to the sporadic forms of the disease. Human molecular genetics, 24, 2023-2034.

493. SPEISER, Z., KORCZYN, A. D., TEPLITZKY, I. \& GITTER, S. 1983. Hyperactivity in rats following postnatal anoxia. Behavioural Brain Research, 7, 379-82.

494. SPILlantini, M. G., CROWTHER, R. A., JAKES, R., HASEGAWA, M. \& GOEDERT, M. 1998. $\alpha$-Synuclein in filamentous inclusions of Lewy bodies from Parkinson's disease and dementia with Lewy bodies. Proceedings of the National Academy of Sciences, 95, 6469-6473.

495. SPILlANTINI, M. G. \& GOEDERT, M. 2013. Tau pathology and neurodegeneration. The Lancet Neurology, 12, 609-622.

496. SPIRES, T. L., ORNE, J. D., SANTACRUZ, K., PITSTICK, R., CARLSON, G. A., ASHE K. H. \& HYMAN, B. T. 2006. Region-specific dissociation of neuronal loss and neurofibrillary pathology in a mouse model of tauopathy. The American journal of pathology, 168, 15981607 .

497. SQUitieri, F., GRIGUOLI, A., CAPELli, G., PORCELlini, A. \& D'AlESSIO, B. 2015. Epidemiology of Huntington disease: first post-HTT gene analysis of prevalence in Italy. Clinical genetics, 89, 367-370.

498. SRULIJES, K., HAUSER, A. K., GUELLA, I., ASSELTA, R., BROCKMANN, K., SCHULTE, C., SOlDA, G., CILIA, R., MAETZLER, W. \& SCHOLS, L. 2013. No association of GBA mutations and multiple system atrophy. European Journal of Neurology, 20, e61-e62.

499. STATS SA, 2012. Census 2011: Census in brief. Statistics South Africa. Pretoria.

500. STATS SA, 2015. Mid-year population estimates. Statistics South Africa. Pretoria.

501. STeEt, R. A., ChUnG, S., WUStMAn, B., POWE, A., DO, H. \& KORNFEld, S. A. 2006. The iminosugar isofagomine increases the activity of N370S mutant acid $\beta$-glucosidase in Gaucher fibroblasts by several mechanisms. Proceedings of the National Academy of Sciences, 103, 13813-13818.

502. STONE, D. L., TAYEBI, N., ORVISKY, E., STUBBLEFIELD, B., MADIKE, V. \& SIDRANSKY, E. 2000. Glucocerebrosidase gene mutations in patients with type 2 Gaucher disease. Human mutation, 15, 181-188.

503. STRACKX, E., VAN DEN HOVE, D., STEINBUSCH, H., STEINBUSCH, H., VLES, J., BLANCO, C. \& GAVILANES, A. 2008. A combined behavioral and morphological study on the effects of fetal asphyxia on the nigrostriatal dopaminergic system in adult rats. Experimental neurology, 211, 413-422. 
504. STRACKX, E., VAN DEN HOVE, D. L., PRICKAERTS, J., ZIMMERMANN, L., STEINBUSCH, H. W., BLANCO, C. E., GAVILANES, A. D. \& VLES, J. H. 2010a. Fetal asphyctic preconditioning protects against perinatal asphyxia-induced behavioral consequences in adulthood. Behavioural brain research, 208, 343-351.

505. STRACKX, E., ZOER, B., VAN DEN HOVE, D., STEINBUSCH, H., STEINBUSCH, H., BLANCO, C., VLES, J., VILLAMOR, E. \& GAVILANES, A. 2010b. Brain apoptosis and carotid artery reactivity in fetal asphyctic preconditioning. Front Biosci (Schol Ed), 2, 781790 .

506. SU, J.H., CUMMINGS,B.J., COTMAN, C.W. 1994. Early phosphorylation of tau in Alzheimer's disease occurs at Ser-202 and is preferentially located within neurites. Neuroreport, 5, 23582362 .

507. SULZER, D. 2007. Multiple hit hypotheses for dopamine neuron loss in Parkinson's disease. Trends in neurosciences, 30, 244-250.

508. SUN, Q.-Y., GUO, J.-F., HAN, W.-W., ZUO, X., WANG, L., YAO, L.-Y., PAN, Q., XIA, K., YAN, X.-X. \& TANG, B.-S. 2013. Genetic association study of glucocerebrosidase gene L444P mutation in essential tremor and multiple system atrophy in mainland China. Journal of Clinical Neuroscience, 20, 217-219.

509. SUN, Y., FLORER, J., MAYHEW, C. N., JIA, Z., ZHAO, Z., XU, K., RAN, H., LIOU, B., ZHANG, W. \& SETCHELL, K. D. 2015. Properties of Neurons Derived from Induced Pluripotent Stem Cells of Gaucher Disease Type 2 Patient Fibroblasts: Potential Role in Neuropathology. PloS one, 10, e0118771.

510. SURMEIER, D. J., GUZMAN, J. N. \& SANCHEZ-PADILlA, J. 2010. Calcium, cellular aging, and selective neuronal vulnerability in Parkinson's disease. Cell calcium, 47, 175-182.

511. SUZUKI, M., FUJIKAKE, N., TAKEUCHI, T., KOHYAMA-KOGANEYA, A., NAKAJIMA, K., HIRABAYASHI, Y., WADA, K. \& NAGAI, Y. 2015. Glucocerebrosidase deficiency accelerates the accumulation of proteinase K-resistant $\alpha$-synuclein and aggravates neurodegeneration in a Drosophila model of Parkinson's disease. Human molecular genetics, 24, 6675-6686.

512. SWAMI, M., HENDRICKS, A. E., GILLIS, T., MASSOOD, T., MYSORE, J., MYERS, R. H. \& WHEELER, V. C. 2009. Somatic expansion of the Huntington's disease CAG repeat in the brain is associated with an earlier age of disease onset. Human molecular genetics, 18, 3039-3047.

513. TAKAHASHI, K., HASEGAWA, S., MAEBA, S., FUKUNAGA, S., MOTOYAMA, M., HAMANO, H. \& ICHIYAMA, T. 2014. Serum tau protein level serves as a predictive factor for neurological prognosis in neonatal asphyxia. Brain and Development, 36, 670-675.

514. TAN, M.-S., YU, J.-T. \& TAN, L. 2013. Bridging integrator 1 (BIN1): form, function, and Alzheimer's disease. Trends in molecular medicine, 19, 594-603.

515. TAN, Y. L., GENEREUX, J. C., PANKOW, S., AERTS, J. M., YATES, J. R. \& KELLY, J. W. 2014. ERdj3 Is an Endoplasmic Reticulum Degradation Factor for Mutant Glucocerebrosidase Variants Linked to Gaucher's Disease. Chemistry \& biology, 21, 967-976.

516. TANG, L.-Q., RINGSTEDT, T., PEQUIGNOT, J. \& LAGERCRANTZ, H. 2000. C-fos gene expression in rat brain around birth: effect of asphyxia and catecholamines. Brain research, $852,84-91$.

517. TAPIA-BUSTOS, A., PEREZ-LOBOS, R., VÍO, V., LESPAY-REBOlledO, C., PALACIOS, E., CHITI-MORALES, A., BUSTAMANTE, D., HERRERA-MARSCHITZ, M. \& MORALES, P. 2016. Modulation of Postnatal Neurogenesis by Perinatal Asphyxia: Effect of D1 and D2 Dopamine Receptor Agonists. Neurotoxicity Research, 1-13. 
518. TASSICKER, R. J., TELTSCHER, B., TREMBATH, M. K., COLLINS, V., SHEFFIELD, L. J., CHIU, E., GURRIN, L. \& DELATYCKI, M. B. 2009. Problems assessing uptake of Huntington disease predictive testing and a proposed solution. European Journal of Human Genetics, 17, 66-70.

519. TERAO, Y., FUKUDA, H., UGAWA, Y. \& HIKOSAKA, O. 2013. New perspectives on the pathophysiology of Parkinson's disease as assessed by saccade performance: a clinical review. Clinical Neurophysiology, 124, 1491-1506.

520. TETTAMANTI, G., BASSI, R., VIANI, P. \& RIBONI, L. 2003. Salvage pathways in glycosphingolipid metabolism. Biochimie, 85, 423-437.

521. THAPAR, A., COOPER, M., EYRE, O. \& LANGLEY, K. 2013. Practitioner review: what have we learnt about the causes of ADHD? Journal of Child Psychology and Psychiatry, 54 $3-16$.

522. THOMAS, R., SANDERS, S., DOUST, J., BELLER, E. \& GLASZIOU, P. 2015. Prevalence of attention-deficit/hyperactivity disorder: a systematic review and meta-analysis. Pediatrics, 135, e994-e1001.

523. TITOMANLIO, L., FERNÁNDEZ-LÓPEZ, D., MANGANOZZI, L., MORETTI, R., VEXLER, Z. S. \& GRESSENS, P. 2015. Pathophysiology and Neuroprotection of Global and Focal Perinatal Brain Injury: Lessons From Animal Models. Pediatric neurology, 52, 566-584.

524. TÖRÖK, R., ZÁDORI, D., TÖRÖK, N., CSILITY, É., VÉCSEI, L. \& KLIVÉNYI, P. 2016. An assessment of the frequency of mutations in the GBA and VPS35 genes in Hungarian patients with sporadic Parkinson's disease. Neuroscience letters, 610, 135-138.

525. TRAUDT, C. M., MCPHERSON, R. J., BAUER, L. A., RICHARDS, T. L., BURBACHER, T. M., MCADAMS, R. M. \& JUUL, S. E. 2013. Concurrent erythropoietin and hypothermia treatment improve outcomes in a term nonhuman primate model of perinatal asphyxia. Developmental neuroscience, 35, 491-503.

526. TREMBATH, M. K., HORTON, Z. A., TIPPETT, L., HOGG, V., COLLINS, V. R., CHURCHYARD, A., VELAKOULIS, D., ROXBURGH, R. \& DELATYCKI, M. B. 2010. A retrospective study of the impact of lifestyle on age at onset of Huntington disease. Movement Disorders, 25, 1444-1450.

527. TRINH, J., GUELLA, I. \& FARRER, M. J. 2014. Disease penetrance of late-onset parkinsonism: a meta-analysis. JAMA neurology, 71, 1535-1539.

528. TSiRoni, E. E., DASTIRIDOU, A., KATSANOS, A., DARDiOTiS, E., VELIKI, S., PATRAMANI, G., ZACHARAKI, F., RALLI, S. \& HADJIGEORGIOU, G. M. 2012. Perimetric and retinal nerve fiber layer findings in patients with Parkinson's disease. BMC ophthalmology, 12,54 .

529. TSUANG, D., LEVERENZ, J. B., LOPEZ, O. L., HAMILTON, R. L., BENNETT, D. A., SCHNEIDER, J. A., BUCHMAN, A. S., LARSON, E. B., CRANE, P. K. \& KAYE, J. A. 2012. GBA mutations increase risk for Lewy body disease with and without Alzheimer disease pathology. Neurology, 79, 1944-1950.

530. TSUNEMI, T., HAMADA, K. \& KRAINC, D. 2014. ATP13A2/PARK9 Regulates Secretion of Exosomes and $\alpha$-Synuclein. The Journal of Neuroscience, 34, 15281-15287.

531. TSUNEMI, T. \& KRAINC, D. 2014. Zn2+ dyshomeostasis caused by loss of ATP13A2/PARK9 leads to lysosomal dysfunction and alpha-synuclein accumulation. Human molecular genetics, 23, 2791-2801. 
532. TURNER, P. V., BRABB, T., PEKOW, C. \& VASBINDER, M. A. 2011. Administration of substances to laboratory animals: routes of administration and factors to consider. Journal of the American Association for Laboratory Animal Science, 50, 600-613.

533. TZVI, E., MÜNTE, T. F. \& KRÄMER, U. M. 2014. Delineating the cortico-striatal-cerebellar network in implicit motor sequence learning. NeuroImage, 94, 222-230.

534. UEMURA, N., KOIKE, M., ANSAI, S., KINOSHITA, M., ISHIKAWA-FUJIWARA, T., MATSUI, H., NARUSE, K., SAKAMOTO, N., UCHIYAMA, Y. \& TODO, T. 2015. Viable Neuronopathic Gaucher Disease Model in Medaka (Oryzias latipes) Displays Axonal Accumulation of Alpha-Synuclein. PLoS genetics, 11, e1005065-e1005065.

535. UNGETHÜM, U., CHEN, Y., GROSS, J., BJELKE, B., BOLME, P., ENEROTH, P., HELDT, J., LOIDL, C., HERRERA-MARSCHITZ, M. \& ANDERSSON, K. 1996. Effects of perinatal asphyxia on the mesostriatal/mesolimbic dopamine system of neonatal and 4-week-old male rats. Experimental brain research, 112, 403-410.

536. VAILLANCOURT, C. \& BOKSA, P. 1998. Caesarean section birth with general anesthesia increases dopamine-mediated behavior in the adult rat. Neuroreport, 9, 2953-2959.

537. VAILLANCOURT, C. \& BOKSA, P. 2000. Birth insult alters dopamine-mediated behavior in a precocial species, the guinea pig: implications for schizophrenia. Neuropsychopharmacology, $23,654-666$.

538. VAIRO, F., SPERB-LUDWIG, F., WILKE, M., MICHELLIN-TIRELLI, K., NETTO, C., NETO, E. C. \& SCHWARTZ, I. V. D. 2015. Brain-derived neurotrophic factor expression increases after enzyme replacement therapy in Gaucher disease. Journal of neuroimmunology, 278, 190-193.

539. VAN DE BERG, W., KWAIJTAAL, M., DE LOUW, A., LISSONE, N., SCHMitZ, C., FAULL, R., BLOKLAND, A., BLANCO, C. \& STEINBUSCH, H. 2003a. Impact of perinatal asphyxia on the GABAergic and locomotor system. Neuroscience, 117, 83-96.

540. VAN DE BERG, W. D., BLOKLAND, A., CUELlO, A. C., SCHMitZ, C., VREUls, W. STEINBUSCH, H. W. \& BLANCO, C. E. 2000. Perinatal asphyxia results in changes in presynaptic bouton number in striatum and cerebral cortex-a stereological and behavioral analysis. J Chem Neuroanat, 20, 71-82.

541. VAN DE BERG, W. D., KWAIJTAAL, M., DE LOUW, A. J., LISSONE, N. P., SCHMITZ, C., FAULL, R. L., BLOKLAND, A., BLANCO, C. E. \& STEINBUSCH, H. W. 2003b. Impact of perinatal asphyxia on the GABAergic and locomotor system. Neuroscience, 117, 83-96.

542. VAN DE BERG, W. D., SCHMitZ, C., STEINBUSCH, H. W. \& BLANCO, C. E. 2002. Perinatal asphyxia induced neuronal loss by apoptosis in the neonatal rat striatum: a combined TUNEL and stereological study. Exp Neurol, 174, 29-36.

543. VAN DEN HEUIJ, L. G., WASSINK, G., GUNN, A. J. \& BENNET, L. 2016. Using Pregnant Sheep to Model Developmental Brain Damage. Prenatal and Postnatal Determinants of Development, 327-341.

544. VAN DEN HOVE, D., STEINBUSCH, H., SCHEEPENS, A., VAN DE BERG, W., KOOIMAN L., BOOSTEN, B., PRICKAERTS, J. \& BLANCO, C. 2006. Prenatal stress and neonatal rat brain development. Neuroscience, 137, 145-155.

545. VAN DER HARG, J., NÖLLE, A., ZWART, R., BOEREMA, A., VAN HAASTERT, E., STRIJKSTRA, A., HOOZEMANS, J. J. \& SCHEPER, W. 2014. The unfolded protein response mediates reversible tau phosphorylation induced by metabolic stress. Cell death \& disease, 5, e1393. 
546. VAN HANDEL, M., SWAAB, H., DE VRIES, L. S. \& JONGMANS, M. J. 2007. Longterm cognitive and behavioral consequences of neonatal encephalopathy following perinatal asphyxia: a review. European journal of pediatrics, 166, 645-654.

547. VAN Velthoven, C. T., BRACCIOLI, L., WILlEMEN, H. L., KAVELAARS, A. \& HEIJNEN, C. J. 2014. Therapeutic potential of genetically modified mesenchymal stem cells after neonatal hypoxic-ischemic brain damage. Molecular Therapy, 22, 645-654.

548. VAN VELThOVEn, C. T., KAVELAARS, A. \& HEIJNEN, C. J. 2012. Mesenchymal stem cells as a treatment for neonatal ischemic brain damage. Pediatric research, 71, 474-481.

549. VAN VELTHOVEN, C. T., KAVElAARS, A., VAN BEL, F. \& HEIJNEN, C. J. 2010a. Mesenchymal stem cell treatment after neonatal hypoxic-ischemic brain injury improves behavioral outcome and induces neuronal and oligodendrocyte regeneration. Brain, behavior, and immunity, 24, 387-393.

550. VAN VELTHOVEN, C. T., KAVELAARS, A., VAN BEL, F. \& HEIJNEN, C. J. 2010b. Nasal administration of stem cells: a promising novel route to treat neonatal ischemic brain damage. Pediatric research, 68, 419-422.

551. VAN VELTHOVEN, C. T., KAVELAARS, A., VAN BEL, F. \& HEIJNEN, C. J. 2010c. Repeated mesenchymal stem cell treatment after neonatal hypoxia-ischemia has distinct effects on formation and maturation of new neurons and oligodendrocytes leading to restoration of damage, corticospinal motor tract activity, and sensorimotor function. The Journal of Neuroscience, 30, 9603-9611.

552. VANNUCCI, R. C. \& VANNUCCI, S. J. 2005. Perinatal hypoxic-ischemic brain damage: evolution of an animal model. Dev Neurosci, 27, 81-6.

553. VANNUCCI, S. J. \& HAGBERG, H. 2004. Hypoxia-ischemia in the immature brain. Journal of Experimental Biology, 207, 3149-3154

554. VÁZQUEZ-BORSETti, P., PEÑA, E., RICO, C., NOTO, M., MiLleR, N., COHON, D., ACOSTA, J. M., IBARRA, M. \& LOIDL, F. C. 2016. Perinatal Asphyxia Reduces the Number of Reelin Neurons in the Prelimbic Cortex and Deteriorates Social Interaction in Rats. Developmental Neuroscience, 38, 241-250.

555. VelayAti, A., DePAOlO, J., GUPtA, N., CHOI, J. H., MOAVEN, N., WESTBROEK, W., GOKER-ALPAN, O., GOLDIN, E., STUBBLEFIELD, B. K. \& KOLODNY, E. 2011. A mutation in SCARB2 is a modifier in Gaucher disease. Human mutation, 32, 1232-1238.

556. Venerosi, A., CUTUli, D., ChiAROTTI, F. \& CAlAMANDREI, G. 2006. C-section birth per se or followed by acute global asphyxia altered emotional behaviour in neonate and adult rats. Behavioural brain research, 168, 56-63.

557. Venerosi, A., VAlanZANo, A., CiRUlli, F., Alleva, E. \& CAlAMANDREI, G. 2004. Acute global anoxia during C-section birth affects dopamine-mediated behavioural responses and reactivity to stress. Behavioural brain research, 154, 155-164.

558. VITNER, E. B., SALOMON, R., FARFEL-BECKER, T., MESHCHERIAKOVA, A., ALI, M., KLEIN, A. D., PLATT, F. M., COX, T. M. \& FUTERMAN, A. H. 2014. RIPK3 as a potential therapeutic target for Gaucher's disease. Nature medicine, 20, 204-208.

559. VITNER, E. B., VARDI, A., COX, T. M. \& FUTERMAN, A. H. 2015. Emerging therapeutic targets for Gaucher disease. Expert opinion on therapeutic targets, 19, 321-334.

560. VLASSAKS, E., BRUdeK, T., PAKKEnBERG, B. \& GAVILANES, A. W. 2014. Cerebellar Cytokine Expression in a Rat Model for Fetal Asphyctic Preconditioning and Perinatal Asphyxia. The Cerebellum, 13, 471-478. 
561. VLASSAKS, E., GAVILANES, A. W., VLES, J. S., DEVILlE, S., KRAMER, B. W. STRACKX, E. \& MARTINEZ-MARTINEZ, P. 2013a. The effects of fetal and perinatal asphyxia on neuronal cytokine levels and ceramide metabolism in adulthood. Journal of neuroimmunology, 255, 97-101.

562. VLASSAKS, E., STRACKX, E., VLES, J., NIKIFOROU, M., MARTINEZ-MARTINEZ, P., KRAMER, B. W. \& GAVILANES, A. 2013b. Fetal asphyctic preconditioning modulates the acute cytokine response thereby protecting against perinatal asphyxia in neonatal rats. J. Neuroinflammation, 10, 14.

563. VOLPE, J. J. 2009a. Brain injury in premature infants: a complex amalgam of destructive and developmental disturbances. The Lancet Neurology, 8, 110-124.

564. VOLPE, J. J. 2009b. Cerebellum of the premature infant: rapidly developing, vulnerable, clinically important. Journal of child neurology, 24, 1085-1104.

565. VOLPE, J. J. 2009c. The encephalopathy of prematurity - brain injury and impaired brain development inextricably intertwined. Seminars in pediatric neurology. Elsevier, 167-178.

566. VOLPE, J. J. 2012. The Neurological Outcome of Perinatal Asphyxia. Early Brain Damage V1: Research Orientations and Clinical Observations, 151

567. VOLPE, J. J., KINNEY, H. C., JENSEN, F. E. \& ROSENBERG, P. A. 2011. Reprint of "The developing oligodendrocyte: key cellular target in brain injury in the premature infant". International Journal of Developmental Neuroscience, 29, 565-582.

568. VON DER HAAR, T., GROSS, J. D., WAGNER, G. \& MCCARTHY, J. E. 2004. The mRNA cap-binding protein eIF4E in post-transcriptional gene expression. Nature structural \& molecular biology, 11, 503.

569. VOn Elm, E., Altman, D. G., EGGer, M., POCOCK, S. J., GØTZSCHE, P. C. \& VANDENBROUCKE, J. P. 2008. The Strengthening the Reporting of Observational Studies in Epidemiology [STROBE] statement: guidelines for reporting observational studies. Gaceta Sanitaria, 22, 144-150

570. WACHTEL, E. V. \& HENDRICKS-MUÑOZ, K. D. 2011. Current management of the infant who presents with neonatal encephalopathy. Current problems in pediatric and adolescent health care, 41, 132-153.

571. WAKUDA, T., IWATA, K., IWATA, Y., ANITHA, A., TAKAHASHI, T., YAMADA, K., VASU, M. M., MATSUZAKI, H., SUZUKI, K. \& MORI, N. 2015. Perinatal asphyxia alters neuregulin-1 and COMT gene expression in the medial prefrontal cortex in rats. Progress in Neuro-Psychopharmacology and Biological Psychiatry, 56, 149-154.

572. WAKUdA, T., MATSUZAKI, H., SUZUKI, K., IWATA, Y., SHINMURA, C., SUDA, S., IWATA, K., YAMAMOTO, S., SUGIHARA, G. \& TSUCHIYA, K. J. 2008. Perinatal asphyxia reduces dentate granule cells and exacerbates methamphetamine-induced hyperlocomotion in adulthood. PLoS One, 3, e3648.

573. WALDHERR, S., MATA, I., CORNEJO-OLIVAS, M., TORRES, L., MAZZETTI, P., COSENTINO, C., VELEZ-PARDO, C., JIMENEZ-DEL-RIO, M., LOPERA-RESTREPO, F. \& TUMAS, V. 2014. Analysis of the GBA gene in the Latin American research consortium on the genetics of Parkinson's disease (LARGE-PD). Movement Disorders, 25, S447.

574. WALKER, P. A., BEDI, S. S., SHAH, S. K., JIMENEZ, F., XUE, H., HAMILTON, J. A., SMITH, P., THOMAS, C. P., MAYS, R. W. \& PATI, S. 2012. Intravenous multipotent adult progenitor cell therapy after traumatic brain injury: modulation of the resident microglia population. Journal of neuroinflammation, 9, 228. 
575. WALKER, P. A., SHAH, S. K., JIMENEZ, F., GERBER, M. H., XUE, H., CUTRONE, R. HAMILTON, J. A., MAYS, R. W., DEANS, R. \& PATI, S. 2010. Intravenous multipotent adult progenitor cell therapy for traumatic brain injury: preserving the blood brain barrier via an interaction with splenocytes. Experimental neurology, 225, 341-352.

576. WALSH, W. F., BUTLER, D. \& SCHMIDT, J. W. 2015. Report of a pilot study of Cooling four preterm infants $32-35$ weeks gestation with HIE. Journal of neonatal-perinatal medicine, $8,47-51$.

577. WANG, C., CAI, Y., GU, Z., MA, J., ZHENG, Z., TANG, B.-S., XU, Y., ZHOU, Y., FENG, T. \& WANG, T. 2014. Clinical profiles of Parkinson's disease associated with common leucinerich repeat kinase 2 and glucocerebrosidase genetic variants in Chinese individuals. Neurobiology of aging, 35, 725. e1-725. e6.

578. WEINREB, N. J., GOLDBlatT, J., VIllalobOS, J., CHARROW, J., COLE, J. A. KERSTENETZKY, M., VOM DAHL, S. \& HOLLAK, C. 2013. Long-term clinical outcomes in type 1 Gaucher disease following 10 years of imiglucerase treatment. Journal of inherited metabolic disease, $36,543-553$.

579. WEIS, S. N., SCHUNCK, R. V., PETTENUZZO, L. F., KROLOW, R., MATTÉ, C., MANFREDINI, V., MARIA DO CARMO, R. P., VARGAS, C. R., DALMAZ, C. \& WYSE, A. T. 2011. Early biochemical effects after unilateral hypoxia-ischemia in the immature rat brain. International Journal of Developmental Neuroscience, 29, 115-120.

580. WEITZDOERFER, R., GERSTL, N., POLLAK, D., HOEGER, H., DREHER, W. \& LUBEC, G. 2004. Long-term influence of perinatal asphyxia on the social behavior in aging rats. Gerontology, 50, 200-205.

581. WEITZDÖRFER, R., HÖGER, H., BURDA, G., POLLAK, A. \& LUBEC, G. 2008. Differences in hippocampal protein expression at 3 days, 3 weeks, and 3 months following induction of perinatal asphyxia in the rat. Journal of proteome research, 7, 1945-1952.

582. WeitzMAN, J. B., FieTte, L., MATSUO, K. \& YANIV, M. 2000. JunD protects cells from p53-dependent senescence and apoptosis. Molecular cell, 6, 1109-1119.

583. Wenning, G. K., STEFAnOVA, N., Jellinger, K. A., POEWE, W. \& SCHLOSSMACHER, M. G. 2008. Multiple system atrophy: a primary oligodendrogliopathy. Annals of neurology, 64, 239-246.

584. WEXLER, N. S. 2004. Venezuelan kindreds reveal that genetic and environmental factors modulate Huntington's disease age of onset. Proceedings of the National Academy of Sciences of the United States of America, 101, 3498-3503.

585. White, J. K., AUERBACH, W., DUYAO, M. P., VONSATTEL, J.-P., GUSELlA, J. F. JOYNER, A. L. \& MACDONALD, M. E. 1997. Huntingtin is required for neurogenesis and is not impaired by the Huntington's disease CAG expansion. Nature genetics, 17, 404-410.

586. WhitTington, R. A., BRETTEVILle, A., DiCKLER, M. F. \& PLANEL, E. 2013. Anesthesia and tau pathology. Progress in Neuro-Psychopharmacology and Biological Psychiatry, $47,147-155$

587. WIATR, K., SZLACHCIC, W. J., TRZECIAK, M., FIGLEROWICZ, M. \& FIGIEL, M. 2017. Huntington Disease as a Neurodevelopmental Disorder and Early Signs of the Disease in Stem Cells. Molecular Neurobiology, 1-21.

588. WINDER-RHODES, S. E., EVANS, J. R., BAN, M., MASON, S. L., WILLIAMS-GRAY, C. H., FOLTYNIE, T., DURAN, R., MENCACCI, N. E., SAWCER, S. J. \& BARKER, R. A. 2013. Glucocerebrosidase mutations influence the natural history of Parkinson's disease in a community-based incident cohort. Brain, 136, 392-399. 
589. WOOD, N. S., MARLOW, N., COSTElOE, K., GIBSON, A. T. \& WILKINSON, A. R. 2000. Neurologic and developmental disability after extremely preterm birth. New England Journal of Medicine, 343, 378-384.

590. WOODARD, C. M., CAMPOS, B. A., KUO, S.-H., NIRENBERG, M. J., NESTOR, M. W., ZIMMER, M., MOSHAROV, E. V., SULZER, D., ZHOU, H. \& PAULL, D. 2014. iPSCDerived Dopamine Neurons Reveal Differences between Monozygotic Twins Discordant for Parkinson's Disease. Cell Reports, 9, 1173-1182.

591. WU, H., LI, Z., YANG, X., LIU, J., WANG, W. \& LIU, G. 2017. SBDPs and Tau proteins for diagnosis and hypothermia therapy in neonatal hypoxic ischemic encephalopathy. Experimental and Therapeutic Medicine, 13, 225-229.

592. WU, Q., CHEN, W., SINHA, B., TU, Y., MANNING, S., THOMAS, N., ZHOU, S., JIANG, H., MA, H. \& KROESSLER, D. A. 2015. Neuroprotective agents for neonatal hypoxic-ischemic brain injury. Drug Discovery Today, 20, 1372-1381.

593. XU, L., DONG, W., ZHAO, J. \& XU, Y. 2015. Effect of Marine Collagen Peptides on Physiological and Neurobehavioral Development of Male Rats with Perinatal Asphyxia. Marine drugs, 13, 3653-3671.

594. XU, Y.-H., XU, K., SUN, Y., LIOU, B., QUINN, B., LI, R.-H., XUE, L., ZHANG, W., SETCHELL, K. D. \& WITTE, D. 2014. Multiple pathogenic proteins implicated in neuronopathic Gaucher disease mice. Human molecular genetics, 23, 3943-3957.

595. XU, Y., SUN, Y., RAN, H., QUINN, B., WITTE, D. \& GRABOWSKI, G. 2011. Accumulation and distribution of $\alpha$-synuclein and ubiquitin in the CNS of Gaucher disease mouse models. Molecular genetics and metabolism, 102, 436-447.

596. YANG, C.-J., TAN, H.-P. \& DU, Y.-J. 2014a. The developmental disruptions of serotonin signaling may involved in autism during early brain development. Neuroscience, 267, 1-10.

597. YANG, C., RAHIMPOUR, S., LU, J., PACAK, K., IKEJIRI, B., BRADY, R. O. \& ZHUANG, Z. 2013. Histone deacetylase inhibitors increase glucocerebrosidase activity in Gaucher disease by modulation of molecular chaperones. Proceedings of the National Academy of Sciences, 110, 966-971.

598. YANG, C., SWALLOWS, C. L., ZHANG, C., LU, J., XIAO, H., BRADY, R. O. \& ZHUANG, Z. 2014b. Celastrol increases glucocerebrosidase activity in Gaucher disease by modulating molecular chaperones. Proceedings of the National Academy of Sciences, 111, 249-254.

599. YANG, C., WANG, H., ZHU, D., HONG, C. S., DMITRIEV, P., ZHANG, C., LI, Y., IKEJIRI, B., BRADY, R. O. \& ZHUANG, Z. 2015. Mutant glucocerebrosidase in Gaucher disease recruits Hsp27 to the Hsp90 chaperone complex for proteasomal degradation. Proceedings of the National Academy of Sciences, 201424288.

600. YANG, T., ZHUANG, L., FidAlgO, A. M. R., PETRIDES, E., TERRANDO, N., WU, X., SANDERS, R. D., ROBERTSON, N. J., JOHNSON, M. R. \& MAZE, M. 2012. Xenon and sevoflurane provide analgesia during labor and fetal brain protection in a perinatal rat model of hypoxia-ischemia. PLoS One, 7, e37020.

601. YAP, T. L., GRUSChUS, J. M., VELAYATI, A., SiDRANSKY, E. \& LEE, J. C. 2013a. Saposin C Protects Glucocerebrosidase against $\alpha$-Synuclein Inhibition. Biochemistry, 52, 7161-7163.

602. YAP, T. L., GRUSCHUS, J. M., VELAYATI, A., WESTBROEK, W., GOLDIN, E., MOAVEN N., SIDRANSKY, E. \& LEE, J. C. 2011. $\alpha$-Synuclein interacts with glucocerebrosidase providing a molecular link between Parkinson and Gaucher diseases. Journal of Biological Chemistry, 286, 28080-28088. 
603. YAP, T. L., JIANG, Z., HEINRICH, F., GRUSCHUS, J. M., PFEFFERKORN, C. M., BARROS, M., CURTIS, J. E., SIDRANSKY, E. \& LEE, J. C. 2015. Structural features of membrane-bound glucocerebrosidase and $\alpha$-synuclein probed by neutron reflectometry and fluorescence spectroscopy. Journal of Biological Chemistry, 290, 744-754.

604. YAP, T. L., VELAYATI, A., SIDRANSKY, E. \& LEE, J. C. 2013b. Membrane-bound $\alpha-$ synuclein interacts with glucocerebrosidase and inhibits enzyme activity. Molecular genetics and metabolism, 108, 56-64.

605. YASUHARA, T., HARA, K., MAKI, M., MAYS, R. W., DEANS, R. J., HESS, D. C., CARROLL, J. E. \& BORLONGAN, C. V. 2008. Intravenous grafts recapitulate the neurorestoration afforded by intracerebrally delivered multipotent adult progenitor cells in neonatal hypoxic-ischemic rats. Journal of Cerebral Blood Flow \& Metabolism, 28, 1804-1810.

606. YASUHARA, T., MATSUKAWA, N., YU, G., XU, L., MAYS, R., KOVACH, J., DEANS, R., HESS, D., CARROLL, J. \& BORLONGAN, C. 2006a. Transplantation of Cryopreserved Human Bone Marrowderived Multipotent Adult Progenitor Cells for Neonatal Hypoxie-Ischemic Injury: Targeting the Hippocampus. Reviews in the Neurosciences, 17, 215-226.

607. YASUHARA, T., MATSUKAWA, N., YU, G., XU, L., MAYS, R. W., KOVACH, J., DEANS, R. J., HESS, D. C., CARROLL, J. E. \& BORLONGAN, C. V. 2006b. Behavioral and histological characterization of intrahippocampal grafts of human bone marrow-derived multipotent progenitor cells in neonatal rats with hypoxic-ischemic injury. Cell transplantation, 15, 231-238.

608. YE, X., LIAN, Q., ECKENHOFF, M. F., ECKENHOFF, R. G. \& PAN, J. Z. 2013. Differential general anesthetic effects on microglial cytokine expression. PloS one, 8, e52887.

609. YU, L., DERRICK, M., JI, H., SILVERMAN, R. B., WHITSETT, J., VÁSQUEZ-VIVAR, J. \& TAN, S. 2011. Neuronal nitric oxide synthase inhibition prevents cerebral palsy following hypoxia-ischemia in fetal rabbits: comparison between JI-8 and 7-nitroindazole. Developmental neuroscience, 33, 312-319.

610. YU, Y., RUN, X., LIANG, Z., LI, Y., LIU, F., LIU, Y., IQBAL, K., GRUNDKE-IQBAL, I. \& GONG, C. X. 2009. Developmental regulation of tau phosphorylation, tau kinases, and tau phosphatases. Journal of neurochemistry, 108, 1480-1494.

611. YU, Z., SAWKAR, A. R. \& KELLY, J. W. 2007. Pharmacologic chaperoning as a strategy to treat Gaucher disease. FEBS journal, 274, 4944-4950.

612. YU, Z., WANG, T., XU, J., WANG, W., WANG, G., CHEN, C., ZHENG, L., PAN, L., GONG, D. \& LI, X. 2015. Mutations in the glucocerebrosidase gene are responsible for Chinese patients with Parkinson's disease. Journal of human genetics, 60, 85-90.

613. ZESKind, P. S., MCMURRAY, M. S., GARBER, K. A., NEUSPIEL, J. M., COX, E. T., GREWEN, K. M., MAYES, L. C. \& JOHNS, J. M. 2011. Development of translational methods in spectral analysis of human infant crying and rat pup ultrasonic vocalizations for early neurobehavioral assessment. Frontiers in psychiatry, 2, 56.

614. ZHAO, Y., REN, J., PADILLA-PARRA, S., FRY, E. E. \& STUART, D. I. 2014. Lysosome sorting of $\beta$-glucocerebrosidase by LIMP-2 is targeted by the mannose 6 -phosphate receptor. Nature communications, 5 .

615. ZHU, C., XU, F., WANG, X., SHIBATA, M., UCHIYAMA, Y., BLOMGREN, K. \& HAGBERG, H. 2006. Different apoptotic mechanisms are activated in male and female brains after neonatal hypoxia-ischaemia. Journal of neurochemistry, 96, 1016-1027.

616. ZIGDON, H., SAVIDOR, A., LEVIN, Y., MESHCHERIAKOVA, A., SCHIFFMANN, R. \& FUTERMAN, A. H. 2015. Identification of a Biomarker in Cerebrospinal Fluid for Neuronopathic Forms of Gaucher Disease. PloS one, 10, e0120194. 
617. ZIMRAN, A., ALTARESCU, G. \& ELSTEIN, D. 2013. Pilot study using ambroxol as a pharmacological chaperone in type 1 Gaucher disease. Blood Cells, Molecules, and Diseases, $50,134-137$.

618. ZIMRAN, A. \& ELSTEIN, D. 2014. Management of Gaucher disease: enzyme replacement therapy. Pediatric endocrinology reviews: PER, 12, 82-87.

619. ZION, Y. C. \& ROSENBAUM, H. 2015. Quality of life and Gaucher disease. Molecular Genetics and Metabolism, 114, S30.

620. ZOGHBI, H. Y. \& BEAR, M. F. 2012. Synaptic dysfunction in neurodevelopmental disorders associated with autism and intellectual disabilities. Cold Spring Harbor perspectives in biology, 4, a009886

621. ZOKAEI, N., MCNEILL, A., PROUKAKIS, C., BEAVAN, M., JARMAN, P., KORLIPARA, P., HUGHES, D., MEHTA, A., HU, M. T. \& SCHAPIRA, A. H. 2014. Visual short-term memory deficits associated with GBA mutation and Parkinson's disease. Brain, 137, 23032311. 




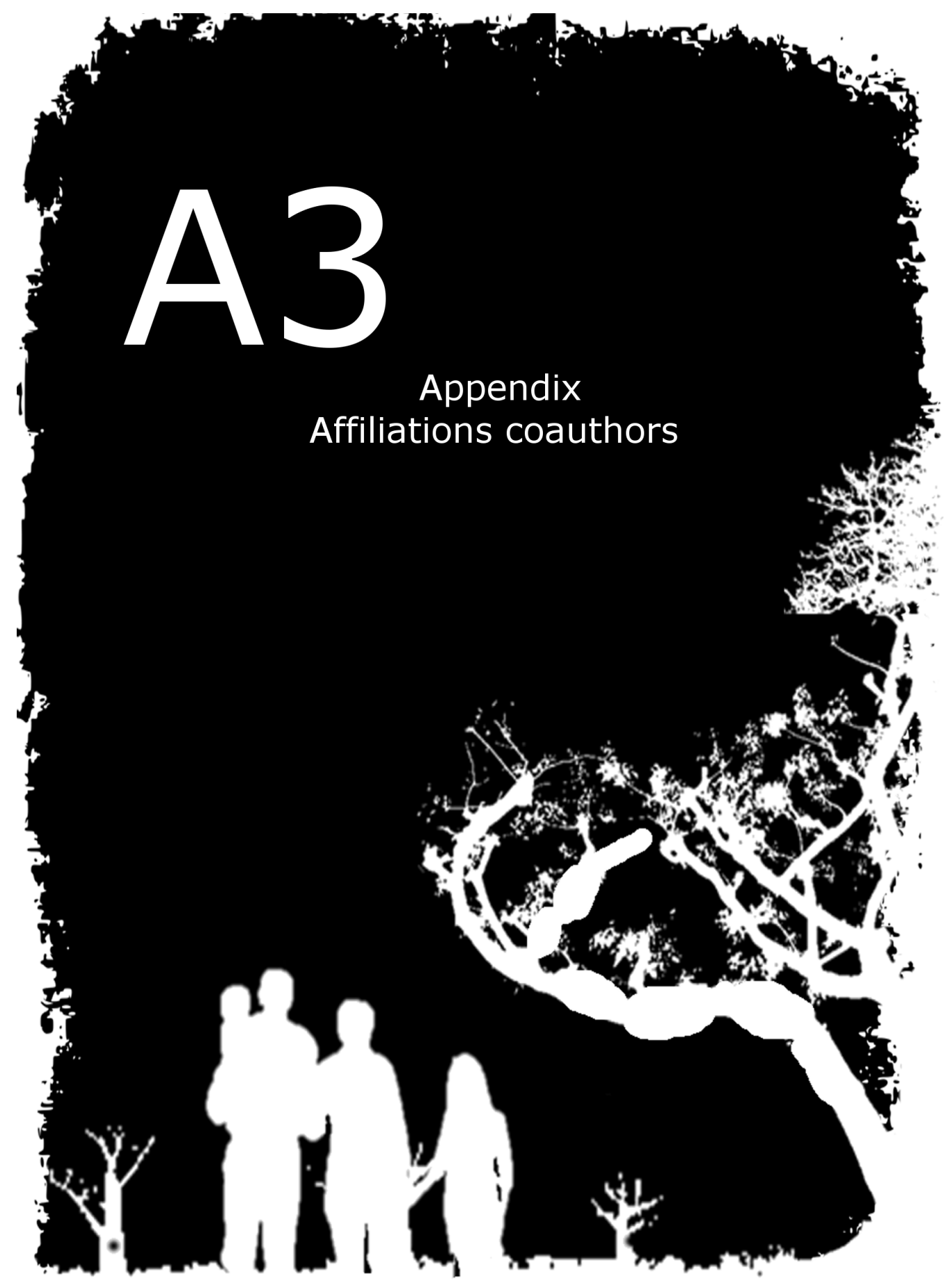




\section{Affiliations of co-authors}

- North-West University, South Africa

- DST/NWU Preclinical Drug Development Platform:

Melinda Barkhuizen, Anne F. Grobler

- Human Metabonomics:

Francois H. van der Westhuizen

- University of the Witwatersrand,South Africa

- Donald Gordon Medical Centre, Department of Neurology: David G. Anderson

- Maastricht University Medical Centre +, The Netherlands

- Department of Pediatrics:

Melinda Barkhuizen, Carlos E. Blanco, Jan de Vente, Imke Engelbertink, A.W. Danilo Gavilanes, Ruth Gussenhoven, Reint K. Jellema, Boris W. Kramer, Matthias Seehase, Wilma. D.J. van de Berg, Fleur van Dijck, Ralph van Mechelen, Marijne Vermeer, Luc Zimmerman

- Department of Methodology and Statistics, CAPHRI: Bjorn Winkens

- Maastricht University, The Netherlands

- School for Mental Health and Neuroscience (MHeNS) and European Graduate School for Neuroscience: Melinda Barkhuizen, A.W. Danilo Gavilanes, Boris. W. Kramer, Dean Paes, Daniel L.A. van den Hove, Harry W.M. Steinbusch, Johan S.H. Vles

- Department of Toxicogenomics, GROW: Marcel van Herwijnen, Danyel Jennen

- Department of Pathology, Cardiovascular Research Institute Maastricht: Jack P.M. Cleutjens

- VU Medical Center, Amsterdam, The Netherlands

- Department of Anatomy and Neurosciences Wilma D.J. van de Berg

- University College London, United Kingdom: 
- Huntington's Disease Centre, UCL Institute of Neurology: Filipe B. Rodrigues, Edward J. Wild

- Institute for Women's Health: Nicola J. Robertson

- Universidad Católica de Santiago de Guayaquil, Ecuador

- Institute of Biomedicine, Facultad de Ciencias Médicas: Peter Chedraui, A.W. Danilo Gavilanes

- University of Lisbon, Portugal

- Laboratory of Clinical Pharmacology and Therapeutics: Filipe B. Rodrigues

- University Medical Center Göttingen, Germany

- Department of Pediatric Cardiology \& Intensive Care Medicine: Matthias Seehase 



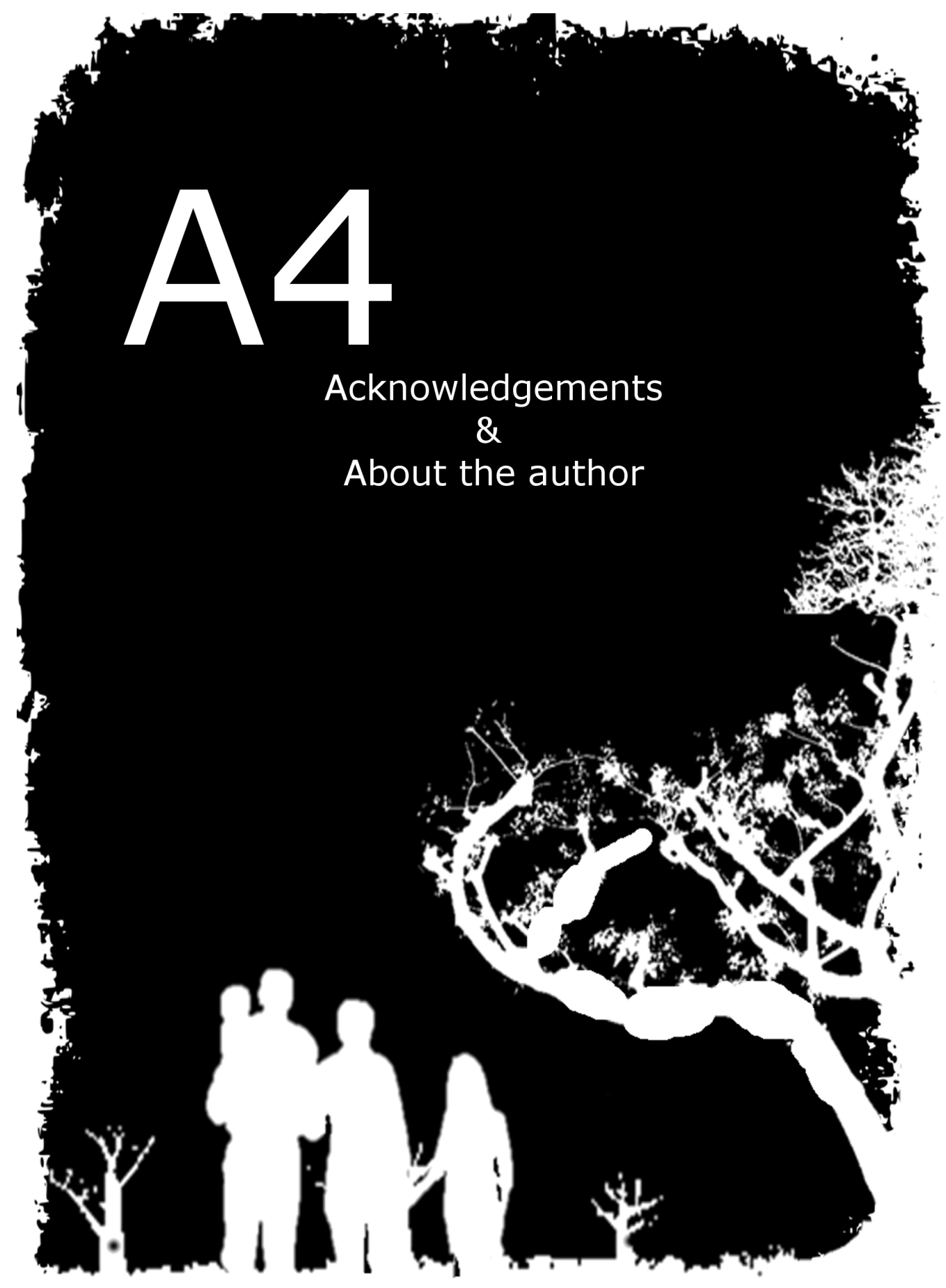




\section{ACKNOWLEDGEMENTS:}

"Wisdom is like a Baobab tree, no one individual can embrace it" - African proverb.

I would like to acknowledge all the individuals and organizations that have been indispensable along the journey that culminated in this $\mathrm{PhD}$.

First and foremost, I would like to thank my promotion team, Prof.dr. Boris Kramer, Dr. Danilo Gavilanes, Prof.dr. Harry Steinbusch and Prof. Anne Grobler who made it possible for me to embark on this wondrous journey that culminated in this $\mathrm{PhD}$.

- Prof.dr. Boris Kramer, thank you for always having a plan B.

- Dr. Danilo Gavilanes, thank you for the guidance and bestowing your rat model upon me.

- Prof. dr. Harry Steinbusch. Thank you for giving me the life-changing opportunity to join MHeNS. Thank you for all the support and career guidance.

- Prof. Anne Grobler. My PhD started in the PCDDP. Thank you for giving me the opportunity to chase my dreams.

\section{Funders and data/reagent sponsors:}

- The National Research Foundation of South Africa, whom financially supported me throughout my postgraduate research (Grant specific reference numbers 89230 and 98217).

- Sistema de Investigación y Desarrollo (SINDE) of the Universidad Católica de Santiago de Guayaquil, Guayaquil, Ecuador (grant No SIU- 319)

- The Stichting Bevordering Kindergeneeskunde 
- The Academy of Science South Africa and the International Brain Research Organization funded the networking opportunities which shaped my career.

- The Lindau Nobel Laureate meetings organizers and Robert Bosch Stiftung. Thank you for the amazing week at the Bodemzee. I was deeply inspired.

- The European Huntington's Disease Network and Enroll-HD initiative, thank you for providing the Huntington's disease epidemiological data used in this study.

- Prof Peter Davies, thank you for the generous gift of the anti-tau antibodies.

- Robert Mays and Bart Vaes at Athersys, thank you for supplying the MAPCs.

\section{The Netherlands:}

- I would not have been able to conduct the work of this thesis by myself. I am immensely grateful for my team of interns Fleur van Dijck, Phyllis van der Ploeg, Imke Engelbertink, Ralph van Mechelen, Marijne Vermeer and my colleague Martin Bustelo. Thank you for all the hard work and for being willing to stay after hours and nights when the experiments required it. Thank you for your enthusiasm. You were an inspiration to me.

- Dr. Daniel van den Hove and Dr. Jos Prickaerts and your team, especially Dean Paes, thank you for the many meetings and helping me make sense of my data. Your expertise contributed immensely to the quality of my thesis.

- Dr. Jack Cleutjens, thank you for your cell counting solutions.

- Marcel van Herwijnen and Dr. Danyel Jennen at toxicogenomics, without your help the transcriptome research would not have been possible.

- The MHENS technicians, Hellen, Marjan, Denise and Marcella and the remaining Expert group members Mario and Ali. Thank you for all the hours spent helping and advising. The excellent technical support was essential to my research.

- The CPV and IVD staff. Richard, Alice, Saskia, Govert and Ramon. Thank you for all the advice and support throughout the ethics and 
testing process. The caretakers, Inger, Mandy, Clarice and Rik, thank you for taking excellent care of my rats.

- Prof. Chris Evelo and Dr. Susan Coort, thank you for teaching me elegant data presentation.

- The team at pediatrics, Nico Kloosterboer, Ruth Gussenhoven, Reint Jellema and Matthias Seehase. Thank you for all your help with the sheep, stem cell and microscopy work. Nico, thank you for supplying the lab equipment I needed and Reint, thank you for the stimulating discussions of my manuscripts.

- Dr. Bjorn Winkens, thank you for the excellent statistical design.

- Prof Hans Vles, thank you for your inputs. I enjoyed our electrophysiology collaboration.

- Dr. Fred van Leeuwen, thank you for your valuable advice on Parkinson's histology and help with antibodies and postmortem tissue.

- Dr. Wilma van de Berg for our collaboration and all the helpful inputs on my writing.

- Dr. Peter Chedraui thank you for your valuable insets on my rodent chapters.

- Laurence and Silvia, thank you for the qPCR advice.

- My current and former office mates. Wouter, thank you for putting up with my talking, the lunch rituals and accepting frikandelbroodjies as a currency for my Dutch translations. The office would have been a lonely place without you. Sandra, thank you for your excellent cutting skills and rodent advice and all the hours spent with my rats. The 'Eye Guys' Christiaan, Suryan and Shuo sharing the office with all of you has been fun.

- All the persons in MHeNS division 3 that I have occasionally run into in the lab or coffee room. Alix, An, Anne, Artemis, Carolin, Cas, Chris, Devon, Fred, Glenn, Gusta, Ines, Koen, Magda, Majed, Marina, Marion, Mark, Maarten, Martin, Nick, Nynke, Roel, Roy, Shenghua, Simone, Sven, Sylvana, Thomas and all the people that I accidentally left out. Thank you for creating a great working atmosphere and making lunch time fun. 
- Rachelle, Peggy, Marie-Therese and Nicole thank you for all the help with the paperwork for this $\mathrm{PhD}$.

- Jesper and Koen, the first two Dutchmen I knew, thank you for introducing me in your culture and your help with the relocation to the Netherlands.

- Lieschen and Claudette, dankie vir die Afrikaanse geselskap as ek die huis mis en vir die stede wat ons saam verken het.

- Sven and Sophie. Thank you for all the food, late-night airport accommodation and interesting genetics debates. You showed me it's possible to have 16000 patients.

- My paranymphs: Gosia Furmanik and Milaine Roet, you two were the first friends I made in Maastricht. Thanks for the paranymphing and all your planning and effort to make this graduation special.

- Milaine thanks for trying to get me to bike ;)

- Gosia and Gerard. Thanks for the memories and gourmet meals and for being there in the middle of the night with my cells.

\section{Russel Square, London:}

- Dr. Filipe Brogueira Rodrigues and Dr. Ed Wild at the UCL Huntington's disease department, thank you for our collaboration and your tremendously helpful insets on Huntington's disease.

- Prof. Nikki Robertson at the Institute for Women's Health thank you for the valuable insights into my manuscripts.

- Prof Huw Morris, Steven Lubbe, Manuella and the team at the UCL Neurogenetics Department. Thank you for collaborating with the South African and introducing me to big data and proper genetics. I had so much fun working with you.

- Dear Filipe and Joanna. Thank you for the friendship, lessons on nonparametric stats, housing and tour guiding and good food. Thank you for keeping me company on my birthday annually. You make London great.

- Yun, thanks for all the words of encouragement during my $\mathrm{PhD}$ writing process. 


\section{South Africa:}

- Dr. Dave Anderson. Words cannot express my gratitude. Thank you for being my first collaborator and all the tremendous support and encouragement throughout my $\mathrm{PhD}$. I still remember the 6 patients you referred to me on my first day with you. Thank you for giving me a second office. Agnes and Busi, thank you for letting me spend time with you in Joburg.

- All the neurologists and doctors and their secretaries who let me live in their waiting rooms and referred patients to me. Especially, Dr. Kritzinger and Nadia, Anina and Theresa; Dr. Meyer and Irene, Dr. Guldenpfennig, Dr. Duvenhage, Dr. Raats and Sr. Martina from Ons Tuis.

- To the patients. Thank you for bravely donating blood or saliva to my research endeavors. Without you there would not have been research.

- Ampath, especially Suzanne Louw. Thank you for generously drawing blood for my study.

- Iain and Angela at the NHLS for the enzyme activity analysis.

- My friends and colleagues at the PCDDP. Janke, thank you for keeping me up to date with everything that is happening back home, Isaac and Urban, thank you for sharing a lab and PCR machine with me. Clinton, thank you for showing me to think like a Zimbabwean, Florentine, Lidja and Jesper, thank you for all the good times and introducing me to your culture and to cheese fondue; Rose, Nicola and Liezl-Marie, thank you for reading through my documents, Adele thanks for the braai's and Zaan thank you for excellent administrative support.

- Prof Francois van der Westhuizen thank you for teaching me how to do molecular biology and Hayley van Dyk thank you for all your excellent technical assistance.

- Prof Jacques Petzer, thank you for giving me a solid foundation in medical chemistry

- Dr. DeWet Wolmarans, thank you for your patience as my pharmacy council tutor

- The team at Inqaba biotech, especially Dr. Christiaan, Dr. Silke, Dr. Misha, Becky and Stella. Thanks for all the advice, patience and technical support. 
- Prof Bardien at Stellenbosch for your advice and reading through my genetics manuscript.

- Dr. Marguerite and Dr. Veronike, thank you for all the years of friendship and for the late-afternoon drives with me help during my recruitment.

- Mia-Louise and Stephen, thanks for all the years of friendship, visiting me and the Maastricht table. I deeply value our friendship.

- Elmarie, Erik, Lucille, Surika and David. Thanks for all the coffees and encouraging words of support throughout this PhD. It was great seeing you when I visited SA.

- My family for all their patience and support, financially and emotionally.

- Die Barkhuizens en die Jordaans: Mamma, pappa, Carla, Fanie, Finney, Este en die res van die familie. Baie dankie dat julle in my geglo het. Die $\mathrm{PhD}$ sou nie moontlik gewees het sonder julle nie.

In their hearts humans plan their course, but the LORD establishes their steps - Proverbs 16:9.

Soli Deo Gloria 

ABOUT THE AUTHOR

\section{Curriculum Vitae}

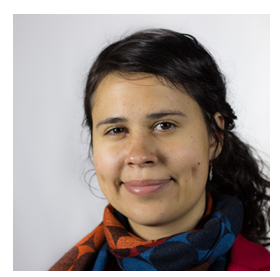

Melinda Barkhuizen was born on 23 November 1990 in Pretoria, South Africa. She has had an interest in movement disorders from a young age. After completing her secondary education at the Hoerskool Waterkloof in Pretoria in 2008, she enrolled for a Bachelor of Pharmacy at the North-West University (NWU) in Potchefstroom, South Africa, which she completed with distinction in 2012. Melinda started her research career with an investigation into drugs which could be repurposed as therapies for Parkinson's disease, under the guidance of Prof. Jacques Petzer at the NWU. She completed her MSc degree (Cum Laude) in 2013. In 2014, she started her PhD trajectory by investigating genetic risk factors for Parkinson's disease under the guidance of Prof. Anne Grobler at the DST/NWU Preclinical Drug Development Platform with financial support from the South African National Research Foundation's Scarce Skills Program. Later in that same year, she met prof.dr. Harry Steinbusch at a course on drug development for Alzheimer's disease, and subsequently enrolled in a double degree $\mathrm{PhD}$ programme at Maastricht University in the Netherlands at the end of 2015. Here she investigated the effect of perinatal injuries on the basal ganglia and tested therapeutic strategies like stem cells to prevent functional deficits after perinatal injuries under the supervision of Prof.dr. Boris Kramer, Dr. Danilo Gavilanes and Prof.dr. Harry Steinbusch. In 2018, Melinda will continue her quest to find a therapy for neurodegenerative diseases in the lab of Dr. Dena Dubal at the Department of Neurology at the University of California San Francisco. 


\section{List of publications}

- Barkhuizen, M., Van de Berg, W.D.J., De Vente, J., Blanco, C.E., Gavilanes, A.W.D., \& Steinbusch, H.W.M. (2017). Nitric Oxide Production in the Striatum and Cerebellum of a Rat Model of Preterm Global Perinatal Asphyxia. Neurotoxicity Research, 31(3), 400-409.

Barkhuizen, M., van den Hove, D.L.A., Vles, J.S.H., Steinbusch, H.W.M., Kramer, B.W., \& Gavilanes, A.W.D. (2017). 25 years of research on global asphyxia in the immature rat brain. Neuroscience \& Biobehavioral Reviews, 75, 166-182.

- Barkhuizen, M., Anderson, D.G., van der Westhuizen, F.H., \& Grobler, A.F. (2017). A molecular analysis of the GBA gene in Caucasian South Africans with Parkinson's disease. Molecular Genetics \& Genomic Medicine, 5(2), 147-156.

- Barkhuizen, M., Anderson, D.G., \& Grobler, A.F. (2016). Advances in GBA-associated Parkinson's disease-Pathology, presentation and therapies. Neurochemistry international, 93, 6-25.

- Barkhuizen, M., Petzer, A., \& Petzer, J. P. (2014). The inhibition of monoamine oxidase by phenformin and pentamidine. Drug research, 64(09), 454-461.

- Barkhuizen, M., Rodrigues, F.B., Anderson, D.G., Winkens, B., REGISTRY investigators of the EHDN, Wild, E.J., Kramer, B.W., \& Gavilanes, A.W.D.(2017). Perinatal insults and neurodevelopmental disorders may impact age of diagnosis of Huntington's disease. Submitted.

- Barkhuizen, M., van Dijck, F., Jellema, R.K., Gussenhoven, R., Engelbertink, I., Cleutjens, J.P.M., Seehase, M., Steinbusch, H.W.M., Zimmermann, L.J., Gavilanes. A.W.D., \& Kramer, B.W. (2017). The influence of anesthetics on tyrosine hydroxylase expression and tau phosphorylation in the hypoxic-ischemic near-term lamb. Submitted.

- Barkhuizen, M., van Mechelen, R., Vermeer, M., Jellema, R.K., Chedraui, P., van Herwijnen, M., Jennen, D., van den Hove, D.L.A., Steinbusch, H.W.M., Robertson, N.J., Kramer, B.W. \& Gavilanes, A.W.D.(2017). The transcriptional effects of multipotent adult progenitor cells in a rat model of preterm perinatal asphyxia. In preparation 
- Barkhuizen, M., van Mechelen, R., Vermeer, M., Jellema, R.K., Chedraui, P., Paes, D., van den Hove, D.L.A., Steinbusch, H.W.M., Robertson, N.J., Kramer, B.W. \& Gavilanes, A.W.D. (2017). Long-term functional outcomes of multipotent adult progenitor cell therapy for asphyxia-related encephalopathy in the immature rat brain. In preparation. 